University of Louisville

ThinkIR: The University of Louisville's Institutional Repository

Electronic Theses and Dissertations

$12-2008$

\title{
The expression and localization of cytoplasmic polyadenylation element binding proteins in the retina.
}

Xiangping Wang

University of Louisville

Follow this and additional works at: https://ir.library.louisville.edu/etd

\section{Recommended Citation}

Wang, Xiangping, "The expression and localization of cytoplasmic polyadenylation element binding proteins in the retina." (2008). Electronic Theses and Dissertations. Paper 1510.

https://doi.org/10.18297/etd/1510

This Doctoral Dissertation is brought to you for free and open access by ThinkIR: The University of Louisville's Institutional Repository. It has been accepted for inclusion in Electronic Theses and Dissertations by an authorized administrator of ThinkIR: The University of Louisville's Institutional Repository. This title appears here courtesy of the author, who has retained all other copyrights. For more information, please contact thinkir@louisville.edu. 


\title{
THE EXPRESSION AND LOCALIZATION OF CYTOPLAMIC POLYADENYLATION ELEMENT BINDING PROTEINS IN THE RETINA
}

\author{
By \\ Xiangping Wang \\ B.S. Peking University \\ Beijing, China, 1997

\begin{abstract}
A Dissertation
Submitted to the Faculty of the

Graduate School of the University of Louisville

in partial Fulfillment of the Requirements

for the Degree of
\end{abstract}

Doctor of philosophy

Department of Anatomical Sciences and Neurobiology

University of Louisville

Louisville, Kentucky

Dec 2008 
THE EXPRESSION AND LOCALIZATION OF CYTOPLAMIC POLYADENYLATION ELEMENT BINDING PROTEINS IN THE RETINA

By

Xiangping Wang

B.S., Peking University, 1997

Beijing, China

A Dissertation Approved on

11.7 .2008

(Date)

By the Following Reading Committee

Nigel G.P. Cooper, Ph.D.

Dissertation Director

Martha Brckford, Ph.D.

Mathew Qiu, Ph.D.

Rita Colella, Ph.D.

Eric Rouchka, Ph.D. 


\section{DEDICATION}

This dissertation is dedicated to my parents

\section{Zhiyan Song}

and

Baolin Wang

for their open-mindedness, unconditional love and ultimate support for education. 


\section{ACKNOWLEDGEMENT}

I would like to give my sincerest thanks to my mentor, Dr. Nigel G.F. Cooper, for his inspirational guidance and ultimate support in all these years. He not only provided me all the financial support, but also gave me tremendous scientific guidance. Many of his insights made this work doable and sustainable. His intelligence, dedication, and openmindedness will always be a role model for me.

I also want to give my deepest gratitude to my committee members: Dr. Martha Bickford, Dr. Mathew Qiu, Dr. Rita Colella, and Dr. Eric Rouchka. Their encouragement, inspiration and support are invaluable to me. My gratitude also goes to my department chair Dr. Fred Roisen, whose always has some wisdom for me at important times of my life. His wisdoms will stay with me forever. 


\begin{abstract}
THE EXPRESSION AND LOCALIZATION OF CYTOPLAMIC POLYADENYLATION ELEMENT BINDING PROTEINS IN THE RETINA
\end{abstract}

\author{
Xiangping Wang
}

Nov 7, 2008

The current status of our knowledge of synaptic plasticity comes largely from studies of the hippocampus and the context of learning and memory. We remain largely ignorant of plasticity in other neural systems and contexts. The molecular basis of plasticity has recently been given new impetus due to the discovery of a local control mechanism which can regulate protein synthesis at stimulated synapses. This involves the use of cytoplasmic polyadenylation binding proteins (CPEBs) to regulate translation. The studies presented here attempt to show that these molecular components are present in the retina, a part of the central nervous system that has been seen, historically, as not plastic.

Methods used: RT-PCR was used to determine the presence of mRNAs in tissue. In situ hybridization and immunofluorescence microscopy were used for localization of mRNAs and proteins respectively. Real-time PCR and Western blots were used for quantifications of mRNA and proteins during postnatal development. A bioinformatics program "CPE detector" and 3' RACE were used to identify potential mRNA targets for CPEB1 in the UTR databases and in the retina respectively. The PAT assay was used to determine the length of poly $(\mathrm{A})$ tails for some potential mRNA targets. Data mining and sequence alignment were used to identify alternatively spliced isoforms of CPEB3. 
Major Results: Our results demonstrated that CPEB1-4 were all present in the retina. The four CPEBs had similar distributions in the inner retina: predominantly in the retinal ganglion cell layer, and to a less extent, in the inner nuclear layer. However, CPEB1 had a laminar pattern in the inner plexiform layer, whereas CPEB3 was diffuse. The presence of CPEB1 was minimal in the outer plexiform layer in contrast to CPEB3. During postnatal development the levels of CPEB1, 3 and 4 were up-regulated; whereas the level of CPEB2 was constant. Potential mRNAs were identified as targets of CPEB1; some mRNA targets demonstrated elongated poly $(A)$ tails at postnatal day7 or day12, consistent with the up-regulation of CPEB1 at these ages. Multiple isoforms, including a novel one, were identified for CPEB3. The alternative splicing of CPEB3 could occur both in the UTRs and in the coding region.

Major Conclusions/Significance: Our data demonstrated that more than one CPEB paralog is present in mouse retina. Potential mRNA targets for CPEB1 were present in the retina and gained elongated poly $(A)$ tail in accordance with the up-regulation of CPEB1 during development. The increases of CPEB1, 3 and 4 during the development indicate a possible role of such CPEBs in synaptogenesis. Continuing up-regulation of CPEB1, 3 and 4 also indicate a role in the adult retina. Alternative splicing in the UTRs of CPEB3 indicates a complex regulation of CPEB3; multiple isoforms of CPEB3 protein indicate the functional complexity of CPEB3. The presence of CPEBs in the retina indicates the existence of a translational control system in the retina.

Future studies: Future studies should focus on the identification of mRNA targets for each CPEB. Such potential targets can be validated using in vitro binding assays to confirm their interaction with CPEB proteins. CPEB can be knocked-down or overexpressed in cultured cells. CPEB knockout mice can be generated for further functional studies. 


\section{LIST OF TABLES}

\section{TABLE}

Table 1 Variants of polyadenylation signal (PAS) and their occurrence.............30

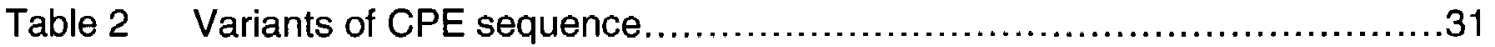

Table 3 Primer sets used for CPEB1-4 and $\beta$-actin in regular RT-PCR ...............74

Table 4 Primers used to identify the alternatively spliced transcripts

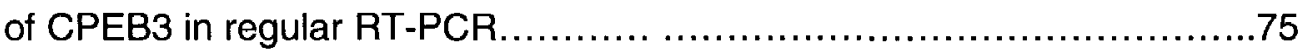

Table 5 Primers used for 3' RACE.............................................

Table 6 Primers used for PAT assay.................................................

Table 7 Gene Expression Assays used for real-time PCR ........................86

Table 8 Match locations of CPEB3 Gene Expression Assay on each

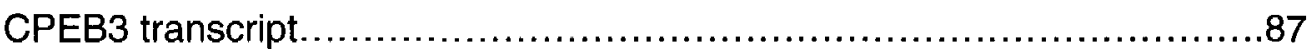

Table 9 Primers used for in situ hybridization.................................. 89

Table 10 Antibodies used for immunofluorescence................................91

Table 11 Antibodies used for Western blots.....................................96

Table 12 CPE-containing mRNAs identified using "CPE Detector" program..........115

Table 13 List of mouse genes with two or more CPEs upstream of PAS (within 250 bp distance) in the 3' UTR

Table 14 Sequences and locations of CPE, PAS, and linker of eight CPEcontaining mRNAs. 


\section{LIST OF FIGURES}

FIGURE

PAGE

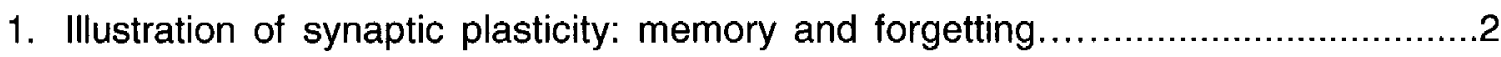

2. Classification and nomenclature of glutamate receptors..............................6

3. Diagrams of two types of ionotropic glutamate receptors: NMDAR and AMPAR.

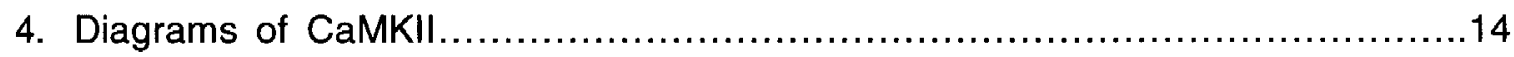

5. MAPK/ERK pathway and synaptic plasticity.......................................19

6. BDNF/TrkB pathway and synaptic plasticity ...................................22

7. PSD - cytoskeletal, scaffolding, adhesion molecules and more $\ldots \ldots \ldots \ldots \ldots \ldots 26$

8. Features of mRNA targets for cytoplasmic polyadenylation.......................32

9. Diagrams of the mechanism for CPEB1-regulated translational control

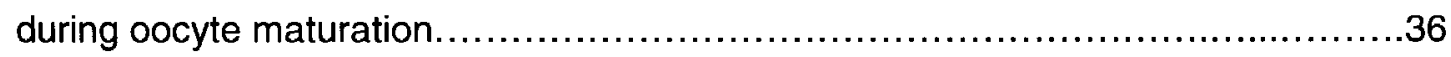

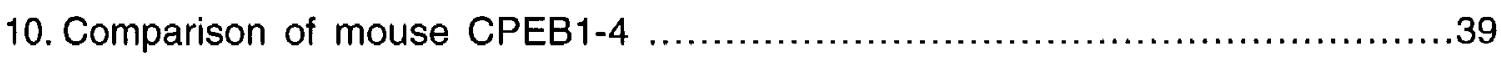

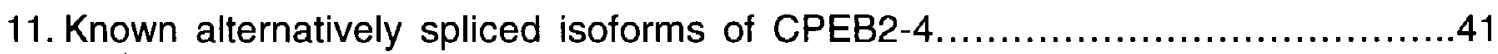

12. RNA secondary structure for CPEB2-4 binding....................................44

13. Cytoplasmic polyadenylation and translation in neurons..........................47

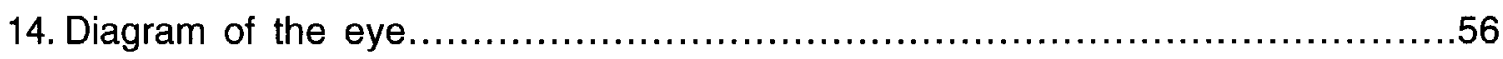

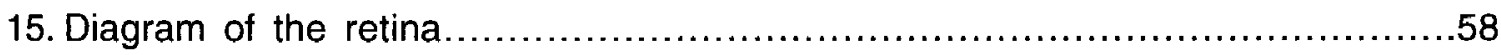

16. Timeframe of synaptogenesis in mouse retina.....................................62

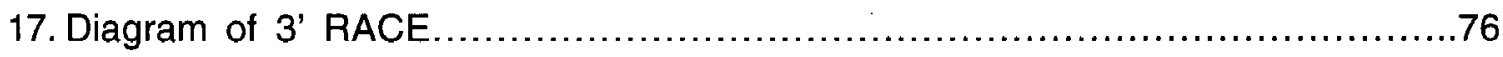

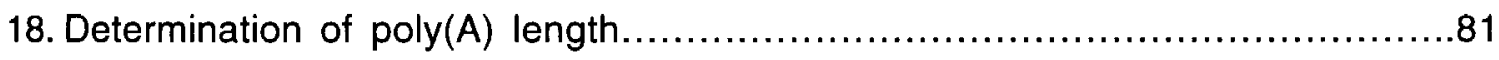




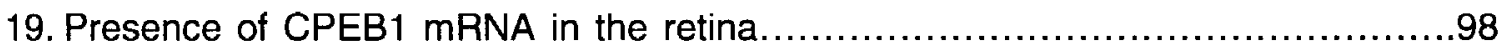

20. Localization of CPEB1 mRNA in the retina...................................... 101

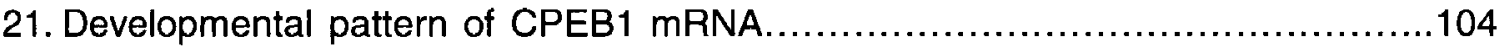

22. Localization of CPEB1 protein - with MAP1a..................................... 106

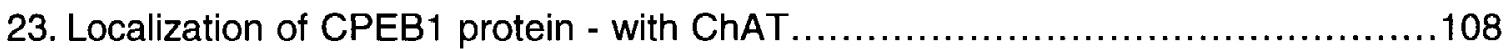

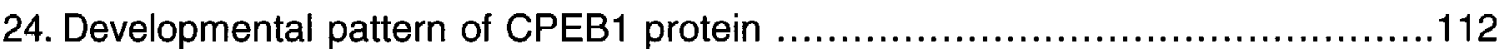

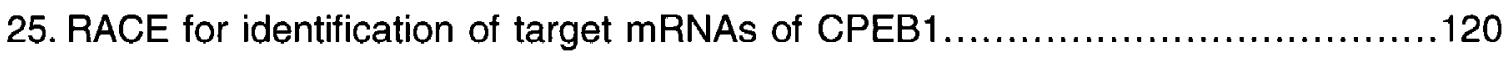

26. LM-PAT assays of potential CPEB1 targets..................................... 123

27. Multiple unique motifs of CPEB3 protein.........................................

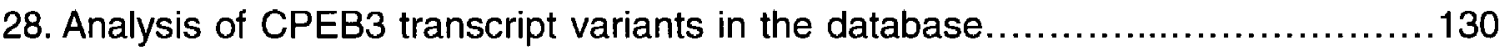

29. Analysis of CPEB3 protein isoforms in the database............................ 132

30. Tissue distribution of CPEB3 transcript variants................................ 135

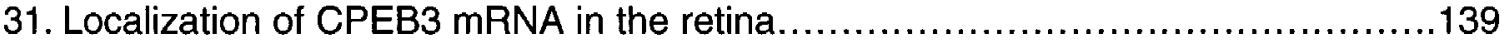

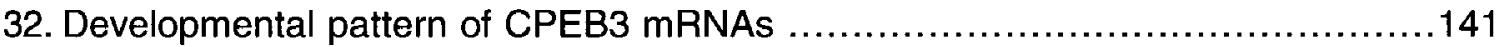

33. Localization of CPEB3 protein- with MAP1a ...................................... 144

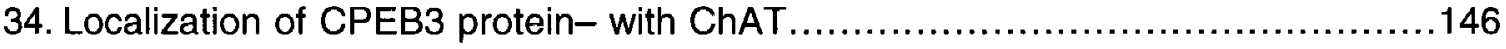

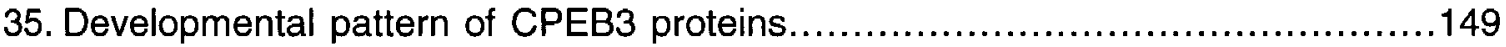

36. Sequence Alignment of CPEB1-4 proteins....................................... 152

37. Sequence Alignment of CPEB2-4 proteins.................................... 155

38. Presence of four CPEBs in the retina.......................................... 158

39. Localization of four CPEB mRNAs in the retina................................ 160

40. Four CPEBs - trends in development...........................................

41. Comparison of the relative abundance of four CPEBs......................... 166 


\section{TABLE OF CONTENTS}

PAGE

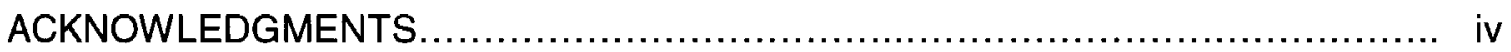

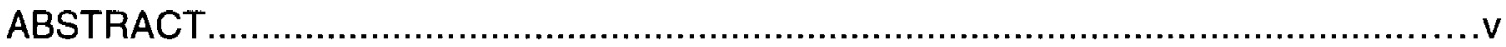

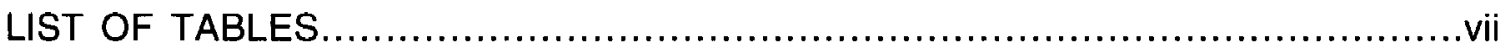

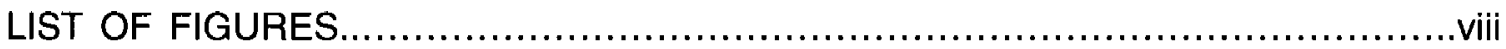

CHAPTER

PAGE

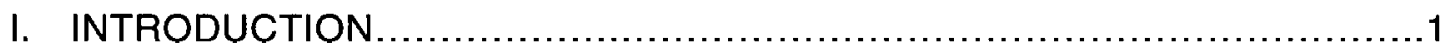

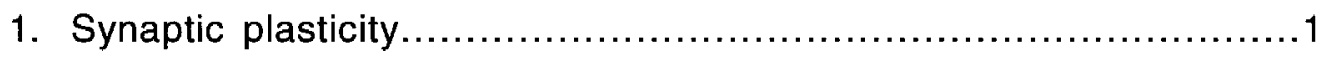

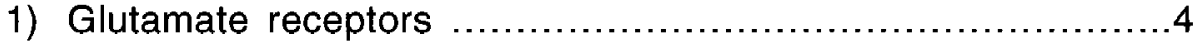

a) NMDAR ...........................................................

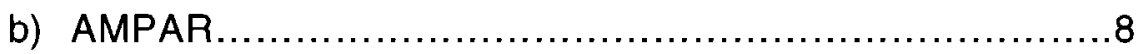

c) Other glutamate receptors...................................12

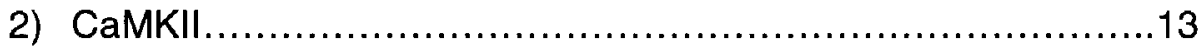

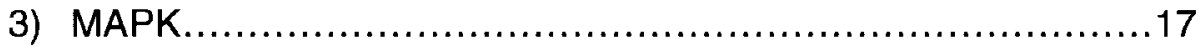

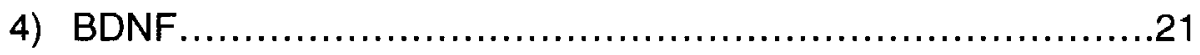

5) Cytoskeletal, scaffolding, and adhesion molecules...............25

2. Cytoplasmic polyadenylation and translational control...................28

1) CPEB1-regulated translational control and its mechanisms.......34

2) Characterization of CPEB2, CPEB3, and CPEB4................38

3. CPEBs and synaptic plasticity...........................................

1) CPEB1 and synaptic plasticity.................................46 
2) CPEB2-4 and synaptic plasticity ...............................50

4. CPEBs and RNA localization.........................................52

1) CPEB1 and mRNA localization in Drosophila.....................52

2) CPEB1 and mRNA transportation in Xenopus oocytes...........53

3) CPEB1 and mRNA localization in the CNS.......................54

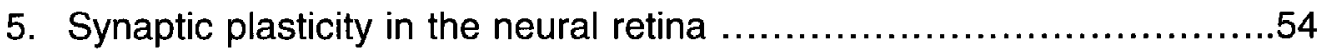

6. CPEBs and CPE-containing mRNAs in the retina.........................61

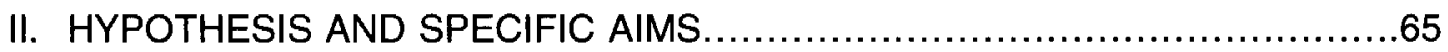

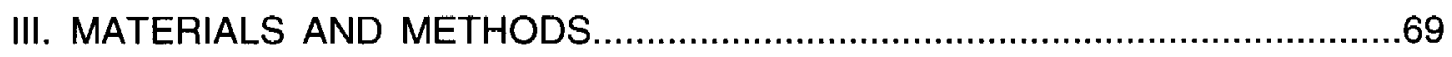

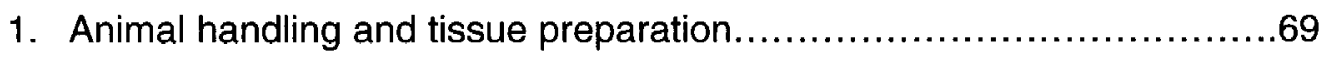

2. Bioinformatic analysis - mRNA targets for CPEB1 .......................70

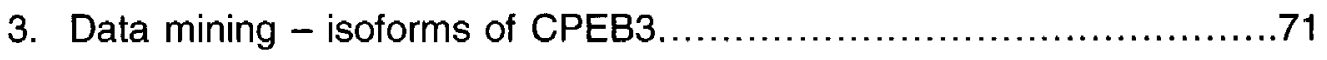

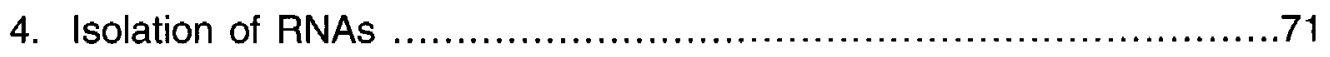

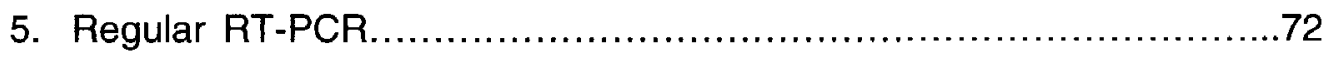

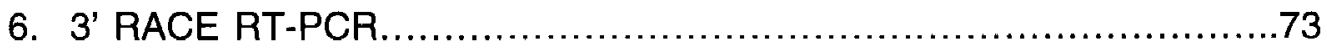

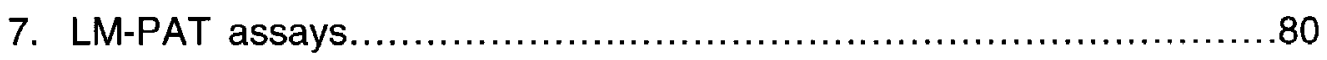

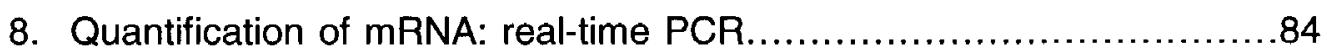

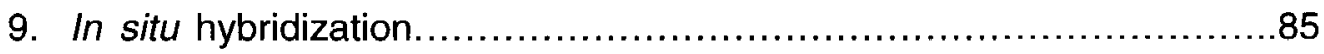

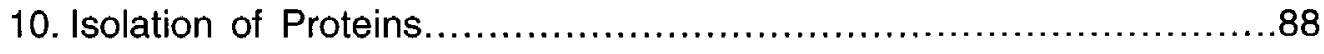

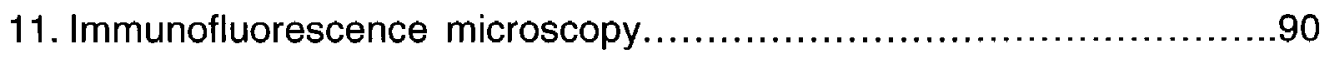

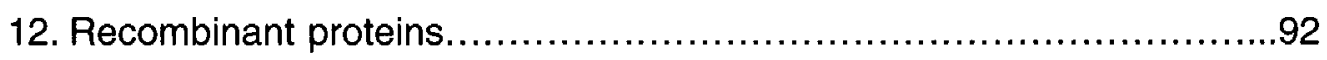

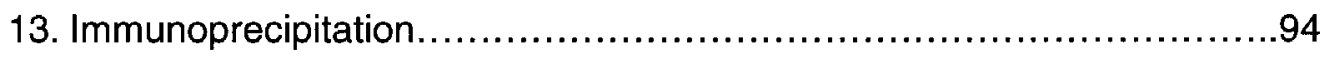

14. Quantification of proteins: Western blotting................................94 
IV. RESULTS

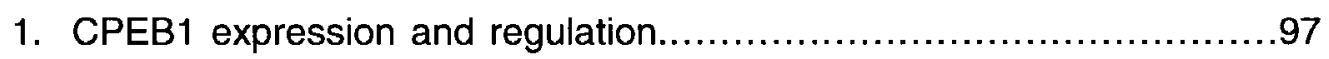

1) Localization of CPEB1 mRNA..................................... 100

2) Developmental pattern of CPEB1 mRNA ….......................100

3) Localization of CPEB1 protein in the retina.........................103

4) Developmental pattern of CPEB1 proteins in the retina...............110

5) Identification of CPE-containing mRNAs in mouse database using bioinformatics program

6) Identification of CPE containing mRNAs from mouse retina using 3' RACE.

7) Polyadenylation of seven CPE-containing mRNAs in diurnal retina.

2. CPEB3 expression, regulation and isoform analysis... 125

1) CPEB3 has multiple unique motifs compared to CPEB1, 2 and $4 \ldots 126$

2) Multiple isoforms of CPEB3 were identified in the database using bioinformatics analysis.

3) Multiple isoforms of CPEB3, including a novel isoform, were experimentally identified in the mouse retina. These isoforms have a wide tissue distribution; and only the $(+69+24)$ variant is selectively expressed among different tissues. .134

4) Localization of CPEB3 mRNA .138

5) $\mathrm{CPEB} 3$ transcription is up-regulated during postnatal development of the retina 138

6) Localization of CPEB3 protein. 143

7) Developmental regulation of CPEB3 proteins. 148

3. CPEB1-4 comparisons. 


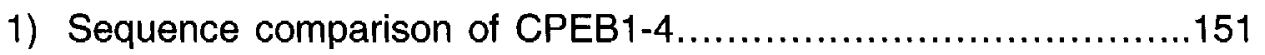

2) All four CPEBs are present in the retina..............................157

3) Cellular localization of CPEB1-4 mRNA ............................157

4) Developmental regulation of CPEB1-4...........................162

5) Relative abundance of four CPEBs...............................165

V. DISCUSSION AND CONCLUSIONS.............................................168

1. Distinct expression patterns and sequence discrepancies indicate functional divergences between members of the CPEB family............169

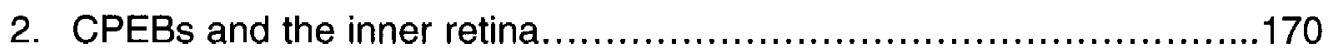

1) CPEB1 and CPEB3 are expressed in the RGC layer, the INL,

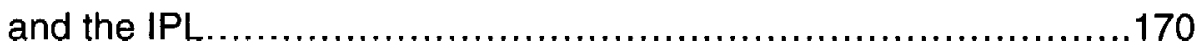

2) The identities of the CPEB positive cells and/or synapses need to be further characterized.............................................171

3) The laminar structure of CPEB1 labeling in IPL may be related

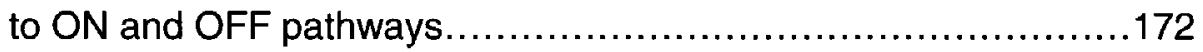

4) The spatial patterns of CPEBs resemble that of some synaptic molecules which are potential targets of CPEBs....................173

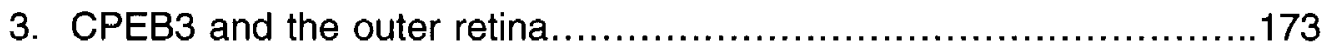

4. CPEB1, CPEB3, CPEB4 and synaptogenesis.........................175

5. CPEB1, CPEB3, CPEB4 and synaptic maturation......................178

6. Synaptic plasticity in the adult retina...................................180

7. Multiple CPEB3 protein isoforms in the retina indicates the complexity of CPEB3 regulation....................................... 182

1) Multiple CPEB3 spliced isoforms are present in the retina...........182 
2) CPEB3 variants provide for a higher level of regulation complexity than has been hitherto recognized.......................184

8. Comparison of CPEB1 and CPEB3..................................185

1) CPEB1 and CPEB3 have different distribution patterns in the retina

2) CPEB1 and CPEB3 may play different roles in the retina ............185

3) There may exist a feedback loop between CPEB1 and CPEB3......186

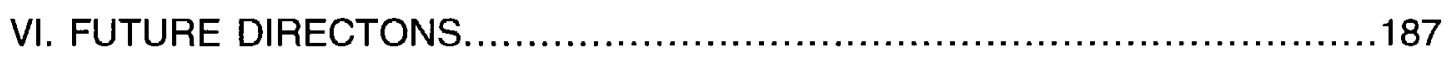

1. In vitro studies: Identification of mRNA targets for each CPEB.........187

1) SELEX screening in combination with bioinformatic tools...........188

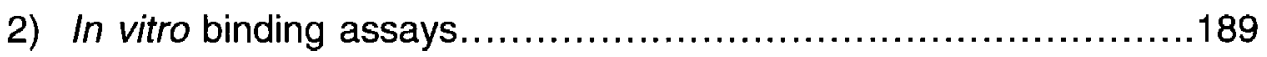

3) In cell knockdown/over-expression analysis.......................189

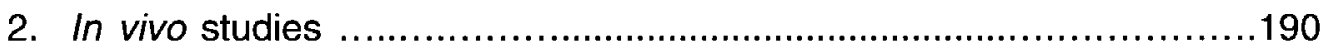

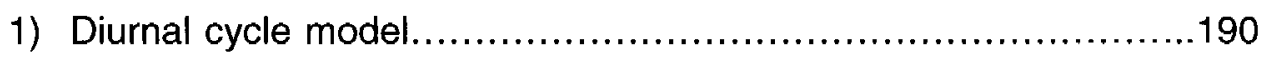

2) Developmental model...............................................191

3) Knockout mouse model...........................................191

4) Photoreceptor degeneration models...............................192

5) Application of kinase inhibitors, neurotransmitter agonists or

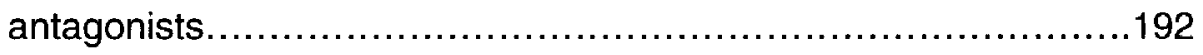

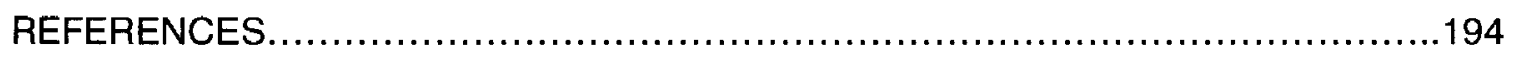

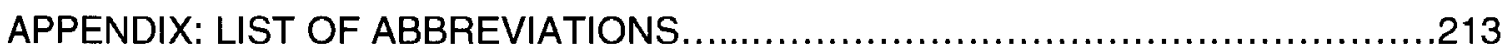

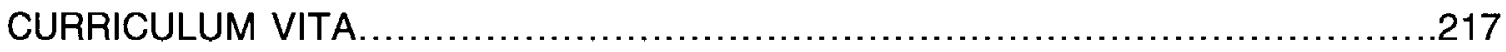




\section{CHAPTER I \\ INTRODUCTION}

\section{Synaptic plasticity}

The old adage "use it or lose it" has been proven correct for many generations. It vividly defines the plasticity in a number of systems in our body, including the central nervous system (CNS). The CNS seems to be endowed with an extraordinary degree of plasticity, one of the important foundations for processes such as development, learning and memory (Silva 2003; Molnar et al. 2002). It is believed that strengthening of existing synapses and formation of new synaptic contacts underlies the formation and consolidation of memory; while the atrophy of synapses in the absence of reinforcement leads to forgetting (figure 1).

Synaptic plasticity is defined as "the ability of synapses to change as circumstances require. They may alter function, such as increasing or decreasing their sensitivity; or they may increase or decrease in actual numbers. This phenomenon is thought to be the main source of the overall plasticity of nervous system pathways" (Dorland's Illustrated Medical Dictionary, $29^{\text {th }}$ edition). Synaptic plasticity portrays the ability of neurons to change their synaptic strength and efficacy of transmission in adaptation to input. Synaptic plasticity can be embodied in several forms, including changes in the amount of neurotransmitters released from presynaptic terminals (Sala et al. 1998; Rosenmund et al. 1993), alteration in the composition and number of receptors /ion channels on the postsynaptic membrane (Yashiro et al. 2005), re-remodeling of synaptic 
Figure 1. Illustration of synaptic plasticity: memory and forgetting (Lüscher et al. 2000) This is a simple illustration for the current understanding of the formation of "memory" with knowledge of current literature. When neurons receive high-frequency stimuli, a series of biochemical and morphological changes occur, which ultimately lead to strengthening of synaptic transmission and increase in the number of synapses. On the other hand, the reverse process - "forgetting" can occur: the synapses undergo "atrophy"- decrease in numbers and in its efficacy in the absence of stimuli (from right to left). 
Figure 1

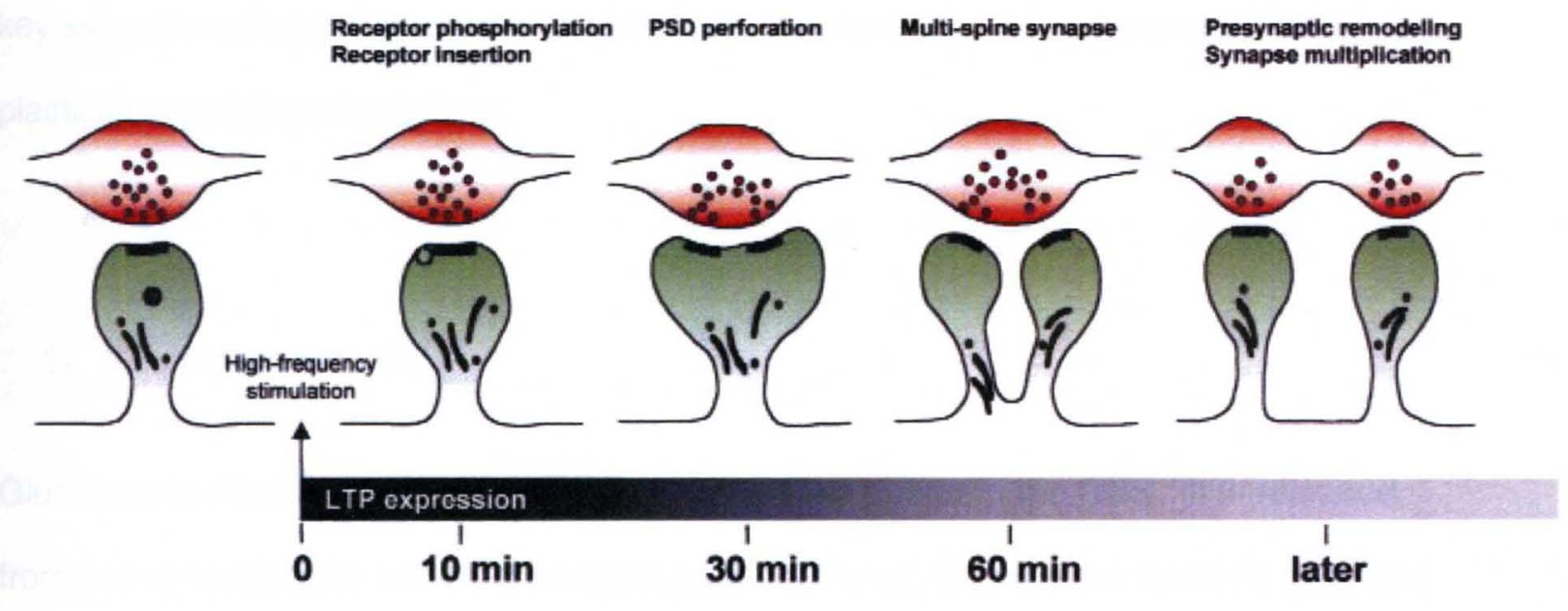


structure (Eliasieh et al. 2007), and an increase or decrease in the number of synapses (Tominaga-Yoshino et al. 2008). The manifestations of synaptic plasticity can be molecular, electrophysiological, morphological, and sometimes, behavioral. Some of the key synaptic molecules that have long been known for their involvement in synaptic plasticity are elaborated below:

1) Glutamate receptors

Glutamate is the most abundant excitatory neurotransmitter in the CNS. It is released from pre-synaptic cells following an appropriate stimulus, crosses the synaptic cleft, and binds to its receptors on post-synaptic cell membrane. Several types of glutamate receptors are present on the post-synaptic cell membrane. These receptors are categorized into two major classes: ionotropic glutamate receptors (iGluR), which are ion channels gated by the ligand, and metabotropic glutamate receptors (mGluR), which are coupled to secondary messenger pathways. The iGluRs are further divided into three classes, named for the glutamate antagonist they respond to: N-methyl-Daspartate receptor (NMDAR), a-amino-3-hydroxy-5-methyl-4-isoxazole propionate receptor (AMPAR), and kainate receptor (KAR) (figure 2). All of these types of glutamate receptors have been implicated in certain forms of synaptic plasticity (Kullmann and Lamsa 2008; Catania et al. 2007; MacDonald et al. 2006; Genoux and Montgomery 2007; Kim et al. 2008; Isaac et al. 2007; Lau and Zukin 2007; Shepherd and Huganir 2007; Bortolotto et al. 2005), with the best-established being NMDAR and AMPAR. 


\section{a) NMDAR}

NMDARs are multimeric protein complexes composed of subunits named NR1, NR2 and NR3, alternatively referred to as GRIN1-3 (figure 2a, 2b). Eight alternative splicing isoforms of NR1 have been identified with distinct tissue distributions. Four isoforms of NR2 (NR2A-D) are selectively expressed across various cell types, with NR2B being the predominant isoform in neurons. The NR2 subunit possesses the binding site for glutamate and determines the receptor's basic kinetic properties. However, the functional properties of NMDARs arise from the differential combination of NR1, NR2 and NR3. A developmental NR2B-NR2A switch has been characterized in the CNS: NR2B is predominant in early stages, while NR2A gradually outnumbers NR2B in the later stages of development (Liu et al. 2004). The developmental switch from a NR2B to a NR2A dominant form is believed to be a modulator of synaptic plasticity (MacDonald et al. 2006) and NMDA neurotoxicity (Liu et al. 2007b; Zhou and Baudry 2006). The integration of NR3A or NR3B to NR1/NR2 subunits modifies the ion permeability of the receptor, and reduces NMDA-induced currents; co-assembly of NR3A or NR3B with $\mathrm{NR} 1$, in the absence of NR2, forms excitatory receptors that can be activated by glycine alone, but unaffected by glutamate or NMDA (Chatterton et al. 2002; Wada et al. 2006; Tong et al. 2008; Awobuluyi et al. 2007). All NMDA receptor subunits are transmembrane proteins. The intracellular domains of these subunits contain sites for several kinases such as protein kinase $A(P K A)$, protein kinase $C(P K C)$, $\mathrm{Ca}(2+) /$ calmodulin-dependent protein kinases (CaMKs), and tyrosine kinases that regulate the functions of the receptors (Wang et al. 2005; Wang et al. 2006). The intracellular domains also possess the binding sites for a series of structural, adaptor, or scaffolding proteins (Beresewicz 2007). 
Figure 2. Classification and nomenclature of glutamate receptors

a) Glutamate receptors are classified into two major classes: ionotropic receptors (iGluR) which are ion channels associated with receptors, and metabotropic receptors (mGluR) which are coupled to secondary messenger pathways. iGluR is divided into three types depending on the agonists they respond to: NMDA receptors (NMDAR), AMPA receptors (AMPAR), and kainate receptors (KAR). Each ionotropic receptor is multimeric. The types of subunits are listed below for each subtype. mGluR each is composed of one transmebrane protein subunit. The types of subunit for mGluR are also listed.

b) Diagram of iGluR (modified from Kandel et al, 1991). IGluRs are receptors associated with ion channels (top). Each iGluR is composed of multiple (4-5) subunits; each subunit is a transmembrane protein that crosses the membrane four times (bottom).

c) Diagram of mGluR (modified from Kandel et al, 1991). mGluRs are coupled to secondary messenger pathways (top). Each mGluR is formed from one polypeptide, which is a transmembrane protein that crosses the membrane seven times (bottom). 
Figure 2

iGluRs $\left\{\begin{array}{l}\text { NMDARs } \\ \text { AMPARs } \\ \text { KARs }\end{array}\right.$
mGluRs $\left\{\begin{array}{l}\text { Type I (quisqualate) } \\ \text { Type II (DCG-IV) } \\ \text { Type III (AP-4) }\end{array}\right.$

NR1-3 (GRIN1-3)

GluR1-4 (GRIA1-4)

GluR 5-7, KA1, KA2

(GRIK1, GRIK2, GRIK3, GRIK4, GIRK5)

a

iGluR

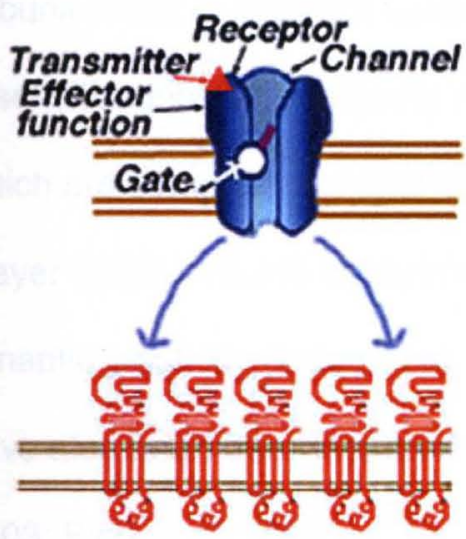

b
mGluR

Extracellular

Cytoplasm

Extracellular

Cytoplasm

mGluR1, mGluR5

mGluR2, mGluR3

mGluR4, mGluR6, mGluR7, mGluR8

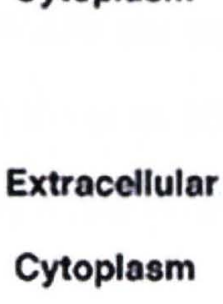

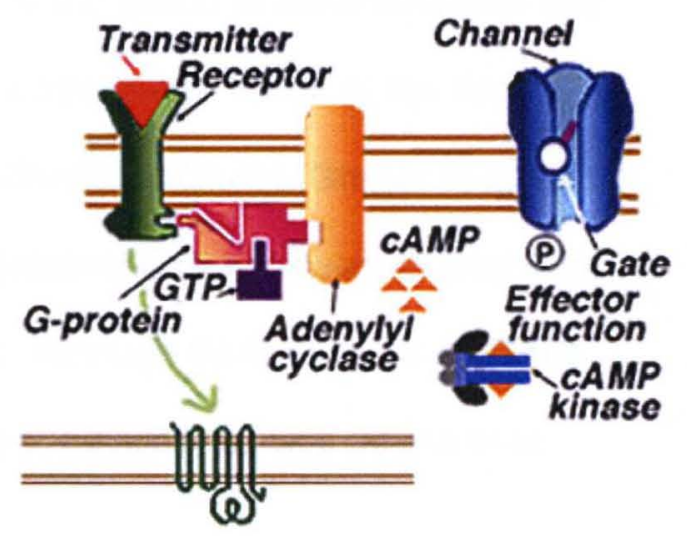

C 
NMDARs (figure $3 a$ ) are $\mathrm{Na}^{+}, \mathrm{K}^{+}$channels which are also highly permeable to $\mathrm{Ca}^{2+}$ (Mayer et al. 1987). NMDARs can be blocked by $\mathrm{Mg}^{2+}$ in a voltage-dependent way or by $\mathrm{Zn}^{2+}$ in a voltage-independent way (Westbrook and Mayer 1987). The activation of NMDARs requires the binding of glutamate and the co-agonist glycine, as well as membrane depolarization. $\mathrm{Ca}^{2+}$ influx via these receptors is believed to evoke glutamate-mediated synaptic plasticity (Baba et al. 2003; Kamiya et al. 2002; Christie et al. 1996; Cummings et al. 1996). A large amount of $\mathrm{Ca}^{2+}$ influx activates CaMKII, which ultimately increases the number of AMPAR on the post-synaptic membrane and elevates the efficacy of the synapses (Poncer et al. 2002). However, excessive $\mathrm{Ca}^{2+}$ influx can also lead to too much excitation (excitotoxicity) and neurotoxicity (Meldrum and Garthwaite 1990).

b) AMPAR

AMPARs are multimers composed of four distinct protein subunits, GluR1-4, alternatively named as GRIA1-4 (figure $2 a, 2 b$ ). Alternative splicing isoforms of each subunit result in different functional properties such as the speed of desensitization and resensitization. The majority of AMPARs consist of a symmetric "dimer of the dimers", which are composed of GluR2 and either of GluR1, GluR3, or GluR4 (Greger et al. 2007; Mayer 2005). GluR2-containing AMPARs are well-established key players in long-term synaptic plasticity (Isaac et al. 2007; Xia et al. 2007), although GluR2-absent AMPARs have also been demonstrated to play a role in synaptic plasticity as well (Meng et al. 2003; Biou et al. 2008).

AMPARs are ion channels permeable to $\mathrm{Na}^{+}$and $\mathrm{K}^{+}$(figure $3 \mathrm{~b}$ ). Their permeability to $\mathrm{Ca}^{2+}$ is determined by the presence or absence of GluR2. AMPARs that lack GluR2 are 
Figure 3. Diagrams of two types of ionotropic glutamate receptors: NMDAR and AMPAR (modified from Kandel et al. 1991)

a) NMDA receptor is selectively agonized by NMDA. The associated channels are permeable to $\mathrm{Na}+, \mathrm{K}+$, and highly permeable to $\mathrm{Ca} 2+$. NMDAR has selective binding sites for glutamate, glycine, Mg2+, $\mathrm{Zn2}+$ and polyamines. The activation of NMDAR requires the binding of both glutamate and glycine. Mg2+ and $\mathrm{Zn} 2+$ inhibit NMDAR in a voltage-dependent and voltage-independent way, respectively. Polyamine is an important modulator of NMDAR.

b) AMPA receptor is selectively agonized by AMPA. The associated channels are permeable to $\mathrm{Na}+$ and $\mathrm{K}+$. The permeability to $\mathrm{Ca} 2+$ is dependent on the subunit composition. GluR2-lacking AMPAR is permeable to Ca2+, while GluR2containing AMPAR is almost impermeable to Ca2+. Subunit composition also modulates the regulation of AMPAR. For example, GluR2-containing AMPAR can be blocked by polyamine in a voltage-dependent manner. 
Figure 3

NMDA receptor

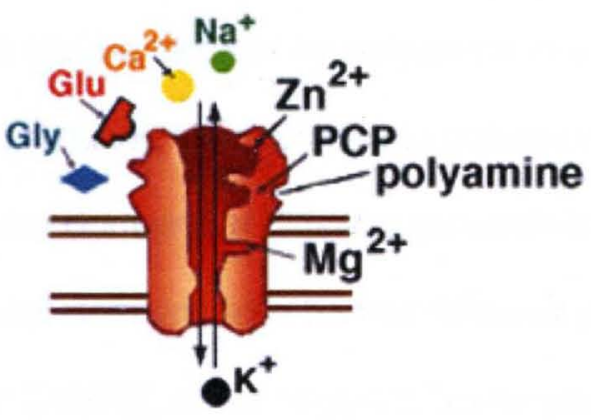

a
AMPA receptor

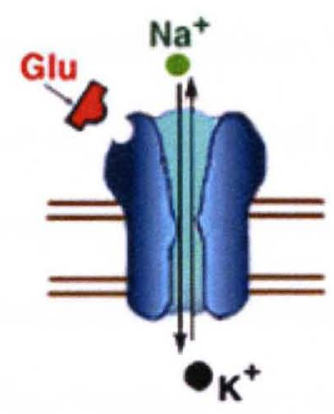

b 
well (figure 5), including a membrane associated form of the G protein Ras (Rosen et al. 1994), $\mathrm{Ca}^{2+} /$ calmodulin activated guanine exchange factors (GEFs), and cytoplasmic protein tyrosine kinase 2 (PTK2) (Orban et al. 1999). The activation of CaMKII can also contribute to the activation of ERK2 by inhibiting an ERK inhibitor synaptic Ras-GTPaseactivating protein (synGAP) (Soderling et al. 2001). A recent study demonstrated that MAPK is required for phosphorylation of cytoplasmic polyadenylation binding protein (CPEB1) during meiosis in Xenopus oocyte, and MAPK directly phosphorylates CPEB1 on four residues (T22, T164, S184, S248), but not on $\$ 174$ which is phosphorylated by aurora kinase (Keady et al. 2007). It is not known whether MAPK can activate CPEB1 in some form of synaptic plasticity in neurons.

\section{4) BDNF}

Brain-derived neurotrophic factor (BDNF) is a member of the neurotrophin family and is known to have an essential role for neuronal survival and differentiation and neurite growth. BDNF also has an important role in memory formation and synaptic plasticity, particularly in glutamatergic neurons. The mature BDNF form preferably binds to and activates TrkB receptor, a member of the receptor tyrosine kinase family. Following secondary messenger signaling cascade, activated TrkB will induce the phosphorylation of glutamate receptor subunits thus affecting the glutamate receptor activity (figure 6) (Carvalho et al. 2008). In contrast, the unprocessed pro-form, proBDNF is coupled to p75 receptor and is possibly involved in cell death (Kenchappa et al. 2006). The mRNAs of BDNF and TrkB are localized to different subcelluler locations including synapses in the hippocampus and visual cortex. The accumulation and synaptic translocation of

BDNF mRNA and proteins was elicited by depolarization stimuli via a pathway involving NMDAR, TrkB and an influx of $\mathrm{Ca}^{2+}$ (Tongiorgi et al. 2006). In the dentate gyrus of rats, 
Figure 6. BNDF/TrkB signaling pathway and synaptic plasticity (Carvalho et al. 2008)

$\mathrm{BDNF} / \mathrm{TrkB}$ regulates the synaptic plasticity at glutamatergic synapses through pre- and postsynaptic targets. Pro-BDNF is secreted from both the pre- and post-synaptic membrane in an activity-regulated way. Mature BDNF generated by extracellular protease digestion of pro-BDNF acts on pre- and postsynaptic TrkB receptors, which is coupled to secondary messenger pathways. In presynaptic compartment, activated TrkB regulates glutamate release; whereas in postsynaptic space, activated TrkB elicits changes in glutamate receptor synthesis and phosphorylation, changes in gene transcription and local translation. These effects of BDNF/TrkB signaling pathway result in the modulation of synaptic efficacy and morphology. 
Figure 6

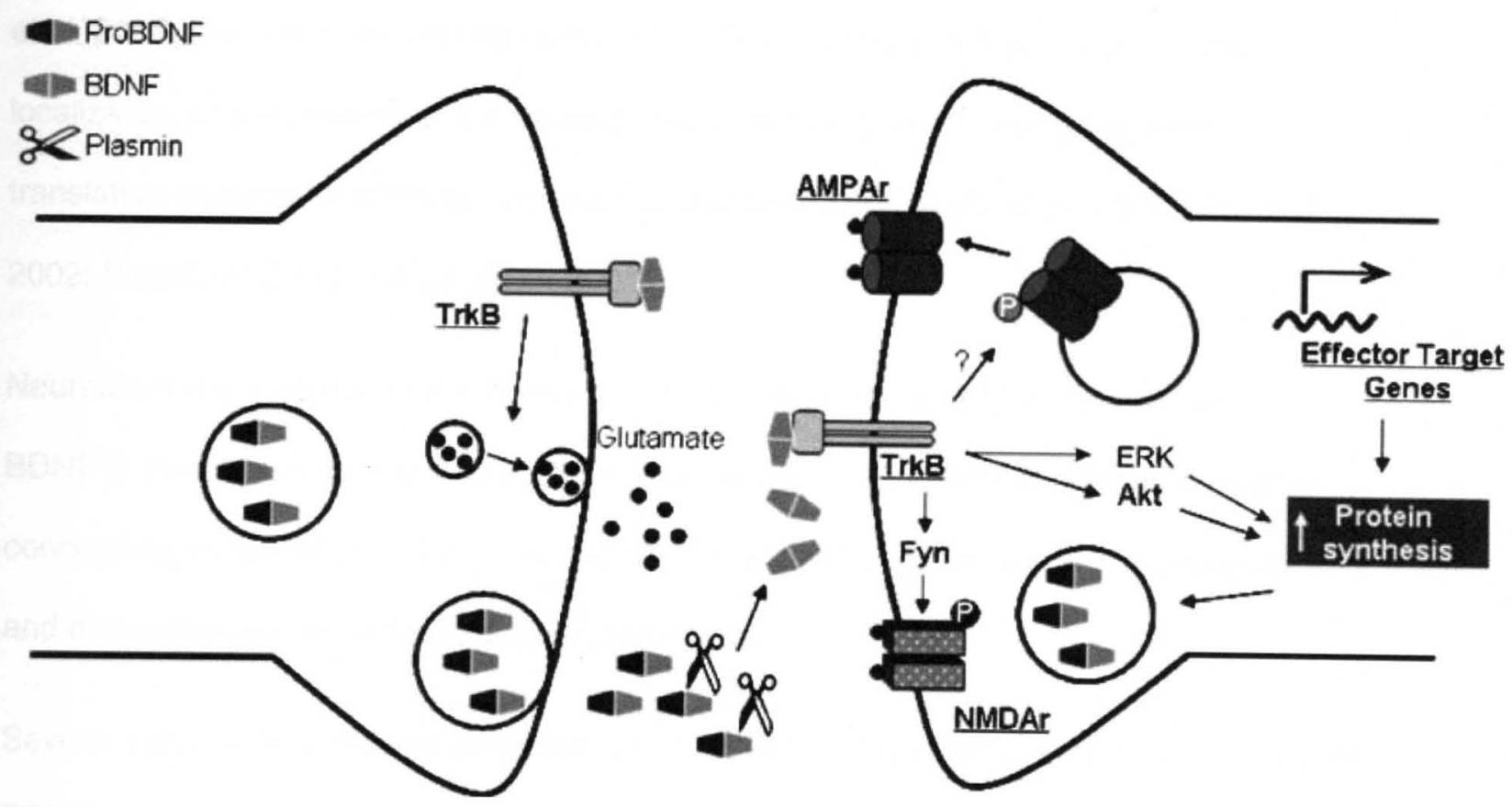


a brief infusion of BDNF induced the transcription and rapid dendrite trafficking of the immediate early gene activity-regulated cytoskeleton-associated protein (Arc), which is required for the local expansion of the actin cytoskeleton during late phase LTP (Soule et al. 2006; Bramham and Messaoudi 2005). BDNF signaling also regulates the localization and activation of the translational machinery, thus driving the local translation of synaptic mRNAs such as Arc and CamKlla (Soule et al. 2006; Yin et al. 2002; Bramham 2008; Aakalu et al. 2001).

Neurotrophin is involved in the formation of neuronal connectivity at several levels. BDNF is involved in axonal and dentritic arborization and activity-dependent synaptic connectivity in retinal ganglion cells (Cohen-Cory and Lom 2004), in the hippocampus and neocortex (Binder and Scharfman 2004).

Several independent studies demonstrated that AMPAR can induce the up-regulation of BDNF in both $\mathrm{Ca}^{2+}$-dependent and $\mathrm{Ca}^{2+}$-independent manners. One study demonstrated that activation of a tyrosine kinase Lyn by AMPAR triggers the MAPK pathway and increases the expression of BDNF (Hayashi et al. 1999).

Two transcript variants of BDNF are produced in the brain. Although both encode for the same protein, one has a short 3' untranslated region (3'UTR) and the other long 3' UTR. The short 3' UTR isoform is restricted to cell somata, whereas the long 3' UTR isoform can be located to synapses. Mutant animals with truncated long 3' UTR manifested impaired dendritic targeting of BNDF mRNA, deficits in dendritic pruning and enlargement, and selective impairment in long-term potentiation in the hippocampus (An et al. 2008). The mechanism by which long 3' UTR isoform of BDNF mRNA is localized and translated in the dendrites is yet to be determined. 
5) Cytoskeletal, scaffolding, and adhesion molecules

Synaptic remodeling requires a dynamic synaptic architecture. Post-synaptic density (PSD) is an electron-dense structure composed of hundreds of proteins, providing a scaffold for the delivery of membrane-bound receptors as well as a matrix for concentrated functional molecules such as kinases, phosphatases, and other key molecules of various signaling pathways involved in synaptic plasticity. Prominent members of the PSD include actin-based cytoskeleton proteins, scaffolding proteins, and adhesion molecules (figure 7) (Boeckers 2006). Cytoskeleton molecules including actin, spectrin, tubulin, microtubule-associated proteins have been isolated from the PSDs. Adhesion molecules such as cadherins are also present at synaptic junctions and play a critical role in synaptic plasticity (Huntley et al. 2002).

The structure of the PSD is dynamic. Its major component, actin, can be a stable structural component or a dynamic filament. Drebrin, a side-binding protein of filamentous actin (F-actin), is located in spines and regulates the recruitment of actin and PSD-95 into filopodia, the precursor of dendritic spines (Takahashi et al. 2003; Mizui et al. 2005; Sekino et al. 2007). The neuronal cell adhesion molecule, $\mathrm{N}$-cadherin, can be synthesized and recruited to the PSD during LTP in the hippocampus.

Insertion of NR2B-containing NMDARs to the synaptic membrane is associated with a dynamic organization of the PSD and an actin-dependent increase in the size of dendritic spines (Mulholland and Chandler 2007). A key cytoskeleton molecule, the Protein Interacting with C-Kinase 1(PICK1), was recently identified as a key regulator of AMPAR trafficking. PICK directly interacts with AMPAR and assists the removal of AMPAR from the synaptic membrane (Hanley 2008). 
Figure 7. PSD - cytoskeletal, scaffolding, adhesion molecules and more (http://www.mcw.edu/FileLibrary/User/mschober/PSD_diagram.jpg)

PSD forms the matrix and platform for key synaptic plasticity molecules to attach and function. PSD is composed of hudreds of different types of proteins, namely, cytoskeletal proteins, scaffolding proteins, adhesion molecules, and other synaptic plasticity-associated molecules. F-actin is one of the major components of PSD. CaMKIla is also highly enriched in PSD. Components of CPEB complexes have also been identified in PSD (ref). PSD provides anchor points for the enrichment of glutamate receptors AMPAR and NMDAR; it also provides a microenviroment for synaptic molecules such as CaMKII and CPEBs to function. 
Figure 7

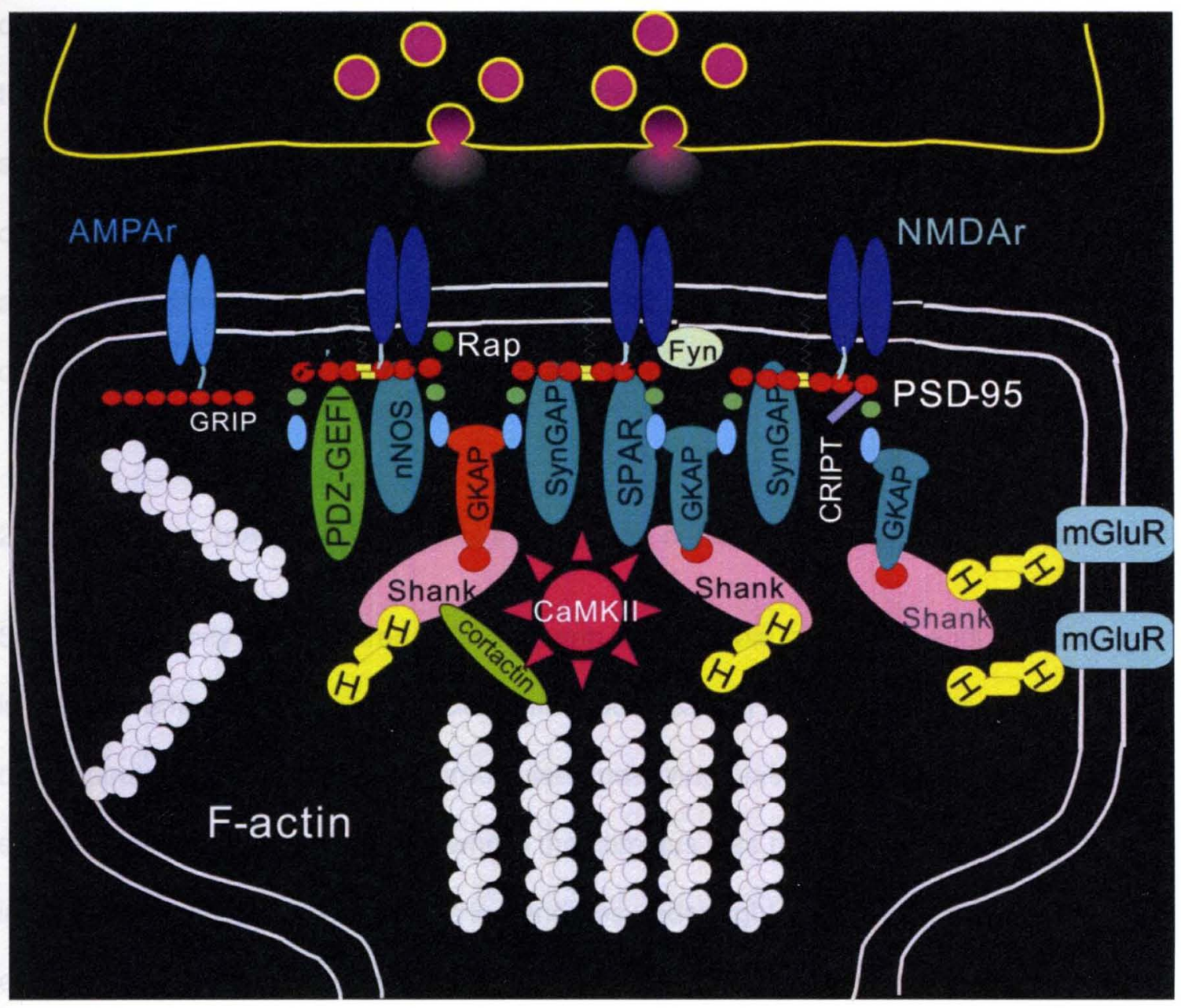


Most of the aforementioned synaptic plasticity molecules are enriched in synapses. A rapid regulation of their translation or synaptic localization needs to be in place in a temporally and spatially specific manner upon the arrival of stimuli. The synthesis and dynamic organization of these molecules is activity dependent, involving $\mathrm{Ca}^{2+}$ influx through activated glutamate receptors and voltage-gated calcium channels at the postsynapse (Schubert and Dotti 2007; Oertner and Matus 2005). For example, activation of NMDAR provides a regulation for the molecular conformational change of cadherin and leads to strengthened adhesion across the synaptic cleft. Nonetheless, the complete mechanisms of such regulation are yet to be delineated.

\section{Cytoplasmic polyadenylation and translational control}

Synaptic plasticity requires transcriptional and translational control for enduring changes in synaptic efficacy (Steward and Schuman 2001; Bading 2000). It needs to be demonstrated that the molecular processes underlying plasticity are temporally and spatially accurate as to when and where the stimulus was received. Recent reports indicate that local protein synthesis in dendrites under tight control may provide an essential mechanism for long term synaptic plasticity. Cytoplasmic polyadenylation may be able to provide a rational explanation of how this can be achieved.

It is well established that all eukaryotic pre-mRNAs undergo polyadenylation in the nucleus before they are exported to the cytoplasm. The newly synthesized pre-mRNA is endonucleolytically cleaved at about 10 nucleotides upstream of the polyadenylation signal (PAS), a hexamer sequence in the 3' UTR. The poly(A) stretch is then added to the newly formed 3' end. The poly(A) tail and its bound proteins are important for termination of transcription, export of the mRNA from the nucleus, and protection of the 
mRNA from degradation by exonuclease. Multiple variants of the PAS sequence have been identified in nature with different occurrences and distinct efficiencies for polyladenylation (table 1).

Cytoplasmic polyadenylation was first identified in eggs and two-cell embryos (Clegg and Piko 1982; Clegg and Piko 1983a; Clegg and Piko 1983b; Wilt 1973), where little RNA transcription activity was detected. A serine protease tissue-type plasminogen activator (t-PA) was translated in a concerted manner in oocytes undergoing meiotic maturation. The mRNA of t-PA accumulates during oocyte growth until the oocyte is fully grown and arrested in meiosis. Resumption of meiosis triggered the polyadenylation of t-PA mRNA in the cytoplasm, which was immediately followed by translation and serves as a novel mechanism for translational regulation (Vassalli et al. 1989; Huarte et al. 1987). Timed expression of pre-stored mRNA was also observed during early embryonic development to initiate mitosis and in some circumstances, to dictate the polarity of the embyo. Such translational activation was accompanied by elongation of the poly(A) tails (Richter 1991; Simon et al. 1992). The same mechanism was later discovered to be employed by the hippocampus and several other parts of the central nervous system, for the acquisition and consolidation of memory, or for long-term synaptic plasticity.

Both a PAS motif and a U-rich motif are required for cytoplasmic polyadenylation of mRNAs (Fox et al. 1989; McGrew et al. 1989). This short U-rich sequence UUUUUAU was later dubbed the "cytoplasmic polyadenylation element" (CPE) (Fox et al. 1989). Several variants of CPE sequence have been identified (McGrew and Richter 1990; Simon et al. 1992) (table 2). CPE is often located upstream of the PAS within a distance of $\sim 10 \mathrm{nt}$ to $100 \mathrm{nt}$, but in some instances overlaps with the PAS (figure 8). Many mRNAs have more than one CPE in their 3' UTR. The variant, the number of copies of 
Table 1. Variants of polyadenylation signal (PAS) and their occurrence

Data were adapted from (Beaudoing et al. 2000). "\% sites" represented the occurrence of the hexamer within $50 \mathrm{nt}$ upstream of potential poly $(A)$ sites. $A$ total number of 5647 putative poly $(A)$ sites (generated by comparing human UTRs to EST sequences) were studied.

\begin{tabular}{cc}
\hline Sequence & $\%$ sites \\
\hline AAUAAA & 58.2 \\
AUUAAA & 14.9 \\
AGUAAA & 2.7 \\
UAUAAA & 3.2 \\
CAUAAA & 1.3 \\
GAUAAA & 1.3 \\
AAUAUA & 1.7 \\
AAUACA & 1.2 \\
AAUAGA & 0.7 \\
AAAAAG & 0.8 \\
ACUAAA & 0.6 \\
\hline
\end{tabular}


Table 2. Variants of CPE sequence

Sequences of CPE variants appeared in the literature are listed below (Fox et al. 1989; McGrew and Richter 1990; Simon et al. 1992).

\begin{tabular}{c}
\hline Sequence \\
\hline UUUUAUU \\
UUUUUAU \\
UUUUAAU \\
$U \cup \cup \cup G \cup U$ \\
\hline
\end{tabular}


Figure 8. Features of mRNA targets for cytoplasmic polyadenylation

The mRNA targets for cytoplasmic polyadenylation have two specific motifs in their 3' UTRs: cytoplasmic polyadenylation element (CPE) and polyadenylation signal (PAS). CPE is often located upstream of PAS, with a gap usually less than $100 \mathrm{nt}$. In some instances, CPE overlaps with PAS. Some mRNA targets have more than one CPE or PAS motifs in their 3' UTRs. The consensus sequences for CPE and PAS are listed. 
Figure 8

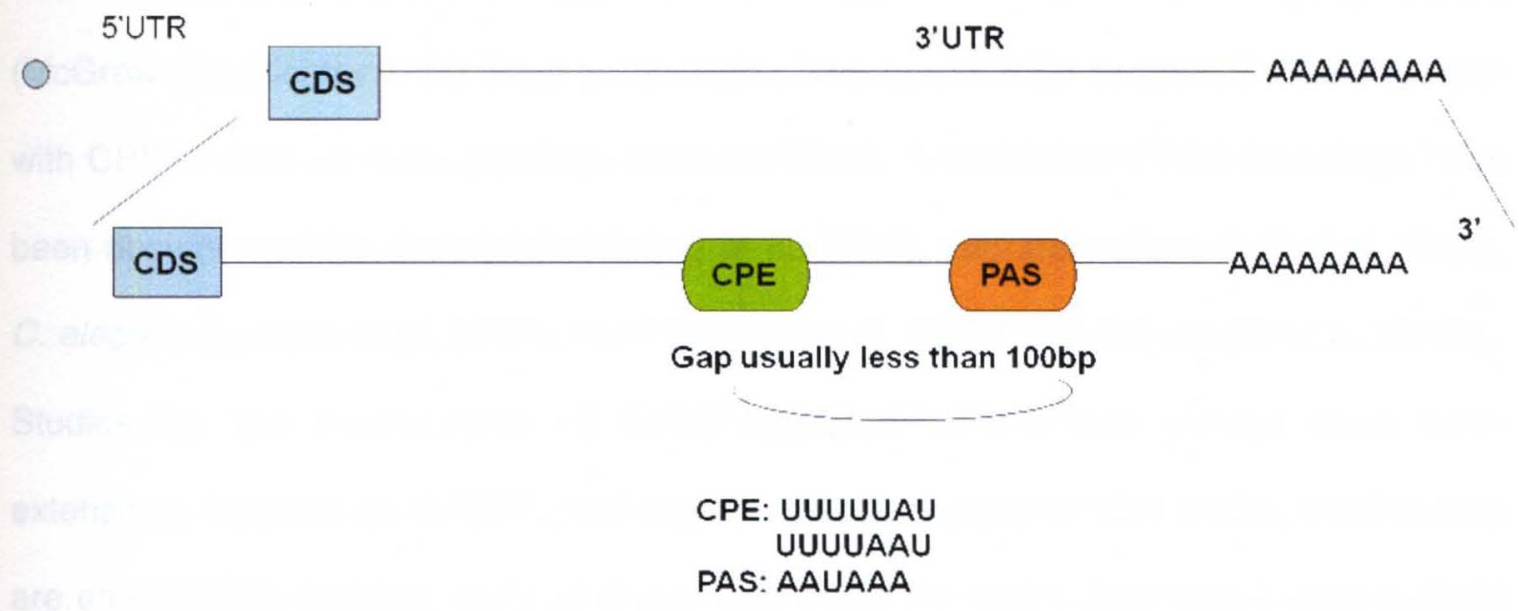


CPE and the distance to the PAS are widely variable for different mRNAs. Such variables could indicate the presence of modulators of the process of translational control.

The CPE binding protein (CPEB) was first identified in 1990 in Xenopus oocytes (McGrew and Richter 1990; Paris et al. 1991). The term CPEB became interchangeable with CPEB1 later as more paralogs were identified. Invertebrate CPEB homologs have been documented for Drosophila (Chang et al. 1999), zebrafish (Bally-Cuif et al. 1998), C. elegans (Luitjens et al. 2000), clam (Walker et al. 1999) and Aplysia (Si et al. 2003a). Studies on the mechanisms of CPEB-regulated translational control have been extensively focused on CPEB1. Although evidence suggested that similar machineries are employed in oocytes, early embryos, and CNS, the major discoveries were derived from work in oocytes, since oocyte maturation is a more facile and efficient model for CPEB1-regulated translational control. The complete mechanisms of CPEB1 regulated polyadenylation/translation is yet to be fully delineated. The current state of our knowledge is summarized below.

1) CPEB1-mediated translational control and its mechanisms

Translation of maternal mRNAs is triggered by progesterone stimulation during oocyte maturation. Activation of progesterone receptors induces a cascade leading to the phosphorylation and activation of CPEB1, which is closely associated with the plasma membrane. One of the first mRNAs that undergo polyadenylation and translation is mos, which codes for a serine/threonine kinase. Mos protein in turn assists the polyadenylation-induced translation of cyclin B1 mRNA. Cyclin B1 together with its 
bound cyclin-dependent kinase 1 (cdk1) will then be responsible for a series of events leading to oocyte maturation (Richter 2007).

CPEB1 has a dual role: a translational activator and a translational inhibitor (figure 9). Under quiescent conditions, unphosphorylated CPEB1 binds to the 3' UTR of mRNA and represses translation. When cells respond to appropriate stimuli, the activation of CPEB1 via phosphorylation or the degradation of CPEB1 removes the repression and allows translation to initiate. The complete mechanism through which CPEB1 regulates polyadenylation is yet to be completely elucidated, but current knowledge indicates that it involves both the 3' and 5' end of the mRNAs. In the dormant state, an adaptor protein, maskin, interacts with both CPEB1 (which binds to CPE in the 3' UTR of the mRNA) and eukaryotic translation initiation factor $4 \mathrm{E}$ (elF4E, which binds to $5^{\prime}$ ' cap of the mRNA) (Stebbins-Boaz et al. 1999). This binding bends the mRNA molecule to bring its 3 ' and 5 ' ends close together. The interaction of maskin to elF4E excludes eukaryotic translation initiation factor $4 \mathrm{G}$ (elF4G) from binding to elF4E. This blocks the assembly of the complete translational machinery. Phosphorylated CPEB is transformed into an activator which gains enhanced affinity for Cleavage and Polyadenylation Specific Factor (CPSF), which in turn recruits Poly(A) Polymerase (PAP) and initiates polyadenylation. The newly synthesized poly $(A)$ tail is quickly bound and protected by $\operatorname{Poly}(A)$ Binding Protein (PABP), which subsequently recruits translational initiation factor elF4G to displace maskin from elF4E (figure 9). This stabilized elF4E-4G interaction enrolls the ribosome for initiation of translation.

This model is supported by multiple studies on oocyte maturation. Maskin, CPSF, PAP and other components have been isolated from the cytoplasmic polyadenylation complex (Mendez et al. 2000; Barnard et al. 2004). 
Figure 9. Diagrams of the mechanism for CPEB1-regulated translational control during oocyte maturation (modified from Groisman et al. 2002)

a) Under dormant state, CPE-containing mRNAs are bound by unphosphorylated CPEB1, which also binds to maskin, an adaptor protein. Maskin in turn binds to elF-4E and prevents the association of elF-4E with elF-4G. In this configuration, mRNA translation is repressed. The cleavage and polyadenylation specific factor (CPSF) may or may not be associated with PAS prior to activation.

b) When CPEB1 is phosphorylated in response to progesterone stimulus, it acquires increased affinity to CPSF. CPSF then binds to PAS and recruits polyadenylation polymerase (PAP), which results in the elongation of the poly(A) tail. Newly synthesized poly $(A)$ tail is bound and protected by poly $(A)$ binding proteins (PABP), which facilitates the recruitment of elF-4G. elF-4G displaces maskin from elF-4E. The association of elF-4E and elF-4G recruits the ribosome for the initiation of translation. 
Figure 9

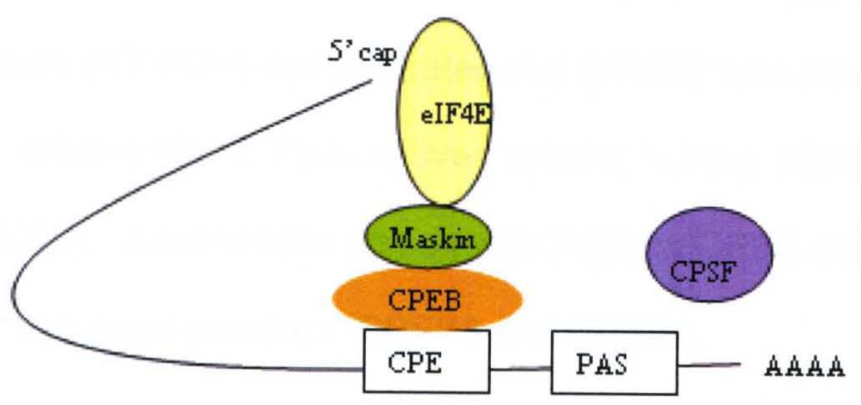

a

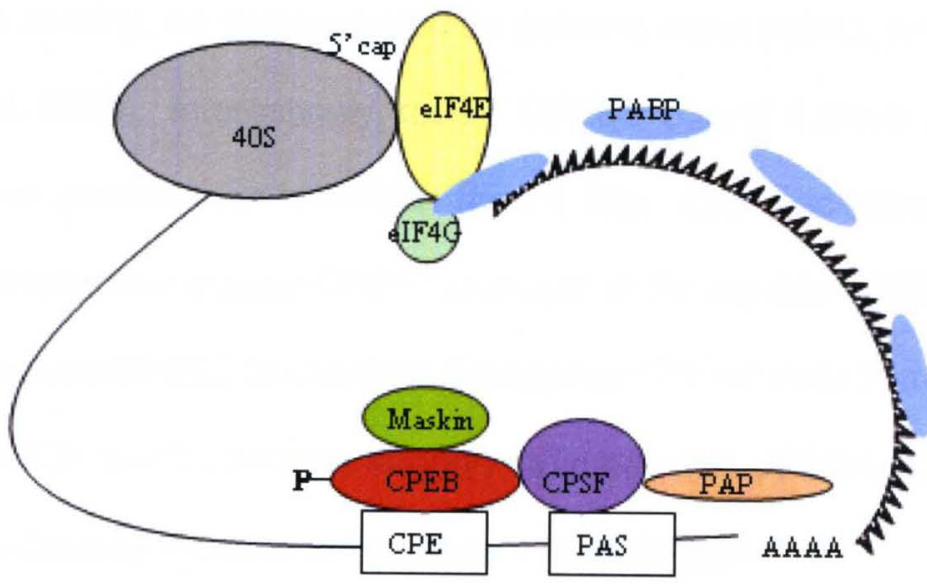

b 


\section{2) Characterization of CPEB2, CPEB3, and CPEB4}

Besides the intensively-studied CPEB1, three additional paralogs of CPEB have been identified in rodents. Mouse CPEB2 was identified as abundant in post-meiotic haploid cells in testes and may have a role in spermatogenesis. Reverse transcriptionpolymerase chain reaction (RT-PCR) demonstrated that CPEB2 was also expressed in cerebrum, cerebellum, salivary gland, thymus, liver, spleen, kidney, intestine, testis, and ovary (Kurihara et al. 2003). A systematic screen with PCR for CPEB genes in mouse brain revealed another two novel paralogs: CPEB3 and CPEB4.

Sequence alignment demonstrated a less homologous $\mathrm{N}$-terminus and a highly conserved C-terminus, among different paralogs and across species (figure 10a). Two RNA recognition motifs (RRF) and a zinc finger region in the $\mathrm{C}$ terminal region are indispensible for RNA binding, as demonstrated by deletion experiments, and are highly conserved (Theis et al. 2003). Interestingly, rodent CPEB2, 3, and 4 share a much higher homology to one another than to CPEB1 (figure 10b). Cross-species comparisons demonstrated that mouse CPEB1 is closer to Drosophila CPEB than to mouse CPEB2, and mouse CPEB2 is closer to Drosophila CPEB2 than to mouse CPEB1 (Theis et al. 2003). Such evidence strongly indicates that CPEB2, 3, and 4 belong to a different subfamily which is rather distant from CPEB1.

Multiple alternatively spliced isoforms have been identified for each of CPEB2, 3 and 4 (Theis et al. 2003). The identified variable regions are the so-called B-region and Cregion (figure 11a, 11b). The significance of the $\mathrm{C}$-region is unknown, but the B-region harbors the recognition sites for PKA, CaMKII, and p70S6 kinases (figure 11c). Unlike CPEB1, CPEB2-4 have no phosphorylation site for aurora kinase. But isoforms of 
Figure 10. Comparison of mouse CPEB1-4

a) The general structure of the CPEB proteins. The $\mathrm{N}$ terminus region is highly variable for different CPEBs, while the C-terminus is highly conserved. Two RNA binding motifs (RRM) together with a Zinc finger domain appear to be indispensable for RNA binding.

b) Homology score of the full-length proteins and of the RNA-recognition motifs (RRM) between CPEB1-4 (Theis et al. 2003). In the overall structure, CPEB2-3, CPEB2-4, and CPEB3-4 demonstrate much higher identity $(69.9,67.7$, and $56.8 \%$ respectively) than with CPEB1. Similarly, in the RNA recognition motifs, CPEB2-4 are almost identical (97-99\%), whereas CPEB1 shares only $44.9 \%$ sequence with the other three. Comparisons were calculated using ClustalW. Scores represent the percentage of identical sequences. 
Figure 10

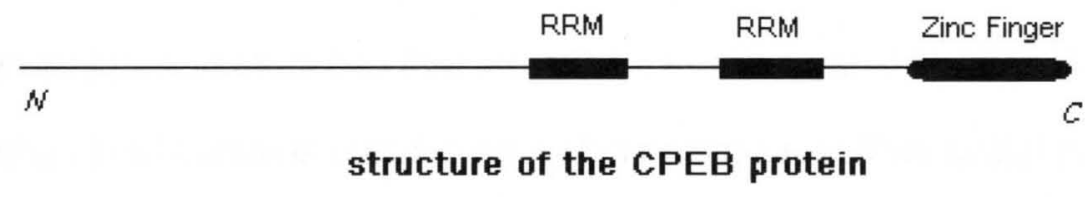

Full length proteins

\begin{tabular}{|c|c|c|c|c|}
\hline & CPEB1 & CPEB2 & CPEB3 & CPEB4 \\
\hline CPEB1 & & 23.8 & 24.8 & 24.6 \\
\hline CPEB2 & 44.9 & & 69.9 & 67.7 \\
\hline CPEB3 & 44.9 & 99 & & 56.8 \\
\hline CPEB4 & 44.9 & 98.5 & 97.5 & \\
\hline
\end{tabular}

RNA recognition motifs

b 
Figure 11. Known alternatively spliced isoforms of CPEB2-4 (modified from Theis et al. 2003)

a) Schematic view of known mouse CPEB3 splice isoforms. (upper) CPEB3 contains two amino acid stretches that are absent in some variants. The B region (hatched box) contains putative phosphorylation sites (filled circle) for PKC, CaMKIla and p70S6 kinases. The $\mathrm{C}$ region is upstream of the $\mathrm{B}$ region. The $\mathrm{C}$ and $\mathrm{B}$ regions can be spliced out individually or concurrently.

b) Schematic view of known mouse CPEB4 spliced isoforms. For explanations, see a). Unlike CPEB3, the $B$ region is not separated from $C$ region in CPEB4.

c) Sequence comparsion of variable regions $B$ and $C$ in mouse CPEB2, 3, and 4 proteins. The $\mathrm{B}$ region is present in some variants of all three CPEBs. Putative phosphorylation sites for PKA, CaMKII and p70S6 kinases are shown above the sequences, and the phosphorylaton site is indicated with an asterisk. The corresponding actual recognition sites are shaded. Note that the phosphorylated serine residue is not in the variable region, but the deletion of $B$ region would disrupt the recognition site. 
Figure 11

mCPEB-3

圆 RRM1 RRM2 Znf

C B

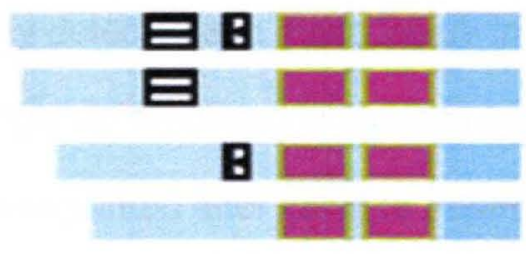

a

\section{mCPEB-4}

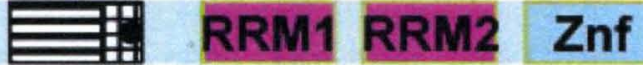

C B

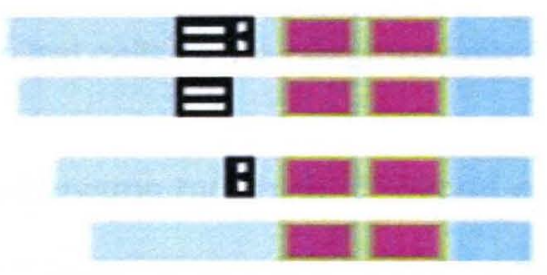

b

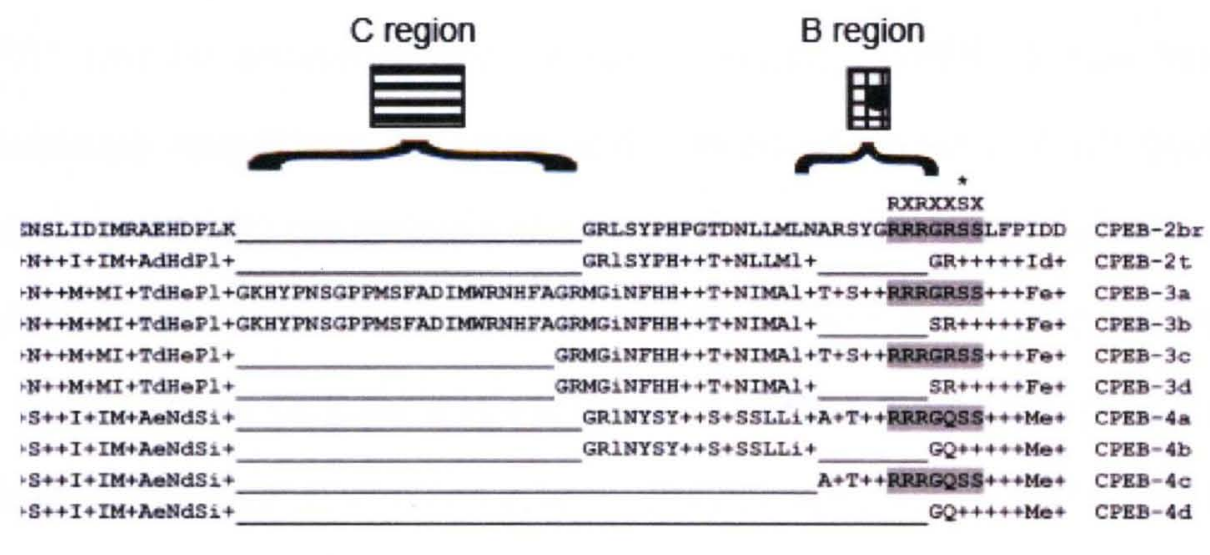


CPEB2-4 containing the B-region can be phosphorylated by PKA, CaMKII and p70S6 kinase (Theis et al. 2003).

Different expression patterns have been characterized for CPEB1-4 in the brain. In the hippocampus, CPEB1, 2 and 4 mRNAs are all present in the CA1/CA3 layers and dentate gyrus. CPEB3 is barely detectable in these layers in the naïve state, but together with CPEB4 it is strongly up-regulated in kainaite-induced seizure. Notably, the CPEB3-4 isforms that were up-regulated after kainaite treatment were the ones containing the B-region (Theis et al. 2003) which possesses the phosphorylation site for PKA, CaMKII and p70S6 kinases. Both CPEB3 and CPEB4 were strongly detected in post-synaptic density factions (Huang et al. 2006). In the cerebellum, both CPEB3 and CPEB4 were demonstrated in the granule cells. However, only CPEB3 appeared in the interneurons, and only CPEB4 appeared in the Pukinje cells. Additionally, CPEB3, but not CPEB4, appeared in the mitral cells of the olfactory bulb (Huang et al. 2006).

Very little is known of the mechanisms by which CPEB2-4 operate. Based on the wide disparity in CPEB1 and CPEB2-4 sequences and differences in their tissue distribution, it is plausible that CPEB2-4 utilize distinct mechanisms from CPEB1. CPEB2-4 may be activated by different kinases such as PKA, CaMKIla, and p70S6 while CPEB1 can be phosphorylated by aurora kinase. CPEB2-4 may have different substrate specificities. Huang and colleagues (Huang et al. 2006) demonstrated that CPEB3 recognizes a U-rich hairpin secondary structure in the 3' UTR of mRNAs (figure 12). The binding of CPEB3 to the hairpin motif and the binding of CPEB1 to the CPE motif is exclusive. CPEB3 but not CPEB1 protein binds to GluR2 mRNA both in vitro and in vivo. The application of CPEB3 siRNA in neurons resulted in an elevated level of GluR2 proteins (Huang et al. 2006). Similar to CPEB1, CPEB3 seemed to be a translational inhibitor of a reporter gene 
Figure 12. RNA secondary structure for CPEB2-4 binding (Huang et al. 2006)

a) A library of artificial RNA was generated from in vitro transcription. The oligonucleotide library composed of random 25-mers (variable sequence) flanked by constant sequences. The mixture was tested for binding to RBD of CPEB4 using systematic evolution of ligands by exponential enrichment (SELEX) method. RNAs that had high binding affinity to the RBD of CPEB4 were listed.

b) Predicted RNA secondary structure necessary for CPEB4 binding. A U-rich loop and a proximal stem upstream were both required. The nucleotides in the boxes indicated the ones protected by CPEB4. This model could be generalized to CPEB2-4, since the RBDs of these are almost identical. 
1914 GAUCGGUGUUUUUUUUGAGGGCGG

1915 UUGGUUCAUGUUUUUUUUGAGGGCGG

1926 GUGUCCGUGUUUUUUUUGAGGGCGG

1908 GUAUUCGUGUUUUUUUUGAGGGCGG

1901 AUUGAGGCUCGAUGUUUUUCAGGAC

1904 AAAGAGGAUUUGUGUUUUUCAGGAC

1929 UAAAAGGAUUCGUGUUUUUCAGGAC

1912 CUGUGGAUCUUGUGUUUUUCAGGAC

1907 UGCAUGUUUUUCGAGUACAGUCCC

2925-2 CGCAUAAUUUUCGAGUAUCAGCCCU

2963-3 CACAUUGUUUUCGAGUUCUAGUCCC

1916 CGCAUACUUUUCGUGUAUUAGUCCG

a

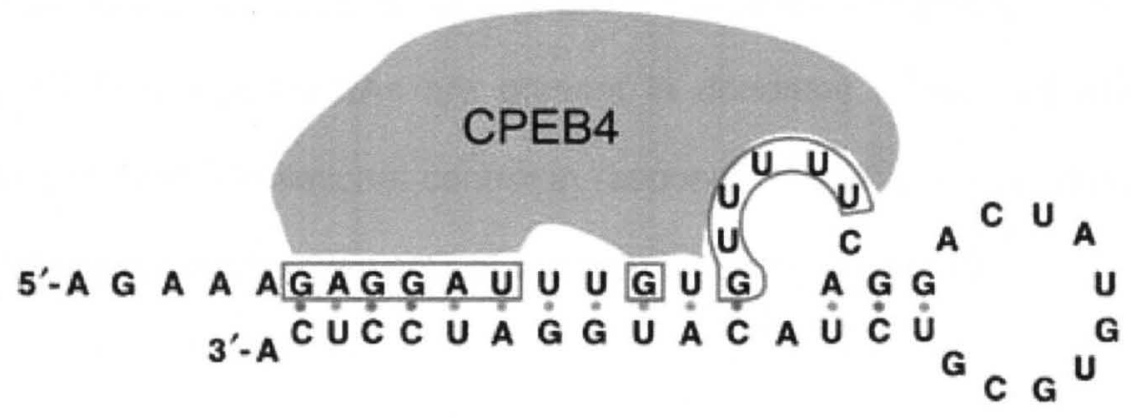

b 
construct in a naive stage, and transforms into an activator upon NMDAR stimulation. CPEB3-mediated translational control appeared to be independent of polyadenylation since it did not bind to CPSF nor did it require the PAS signal (Huang et al. 2006).

\section{CPEBs and synaptic plasticity}

Neurons are highly polarized cells that require differential distribution of proteins and mRNAs to different cellular compartments such as somata, axon and dendrites. A neuron can receive signals from thousands of cells. A dendrite that receives a signal is distinguished from many other dendrites that do not by establishing a "tag" of the stimulated synapse. This "tag" is composed of up-regulated proteins which will affect the way the synapse responds to stimuli in the future. Formation of such synaptic plasticity requires both temporally and spatially regulated translation at the synaptic sites. Many components of the protein synthesis machinery, including polysomes, CPEBs, and mRNAs are present in dendrites. This indicates that CPEB-mediated local translational control in response to synaptic activation could be one of the underlying mechanisms of synaptic plasticity (figure 13).

\section{1) CPEB1 and synaptic plasticity}

Intriguingly, the translational mechanisms employed by CPEB1 in oogenesis and early embryogenesis could be recapitulated in the brain. The CPEB that was most intensively studied for its role in synaptic plasticity is CPEB1. Localized translation at the synaptic sites is one of the essential underlying mechanisms for neuronal processes such as 
Figure 13. Cytoplasmic polyadenylation and translation in neurons (Wells et al. 2000)

Synaptic plasticity induces protein synthesis in neurons. CPEB is localized to synapses and enriched in the postsynaptic density (PSD) in the brain. Activation of NMDAR triggers the phosphorylation of CPEB and initiation of its target mRNAs (ref). This model is supported by the discovery that CPE-containing mRNA, CaMKIla, is enriched in PSD. Its polyadenylation and translation was up-regulated in the visual cortex of dark-reared animals when these animals were exposed to light (Wu et al. 1998). This process was blocked by antagonist of NMDAR or inhibitors of polyadenylation (Kirkwood et al. 1996; Wells et al. 1999). The mechanism of how NMDAR activates CPEB is yet to be determined (as indicated by "?"). 
Figure 13

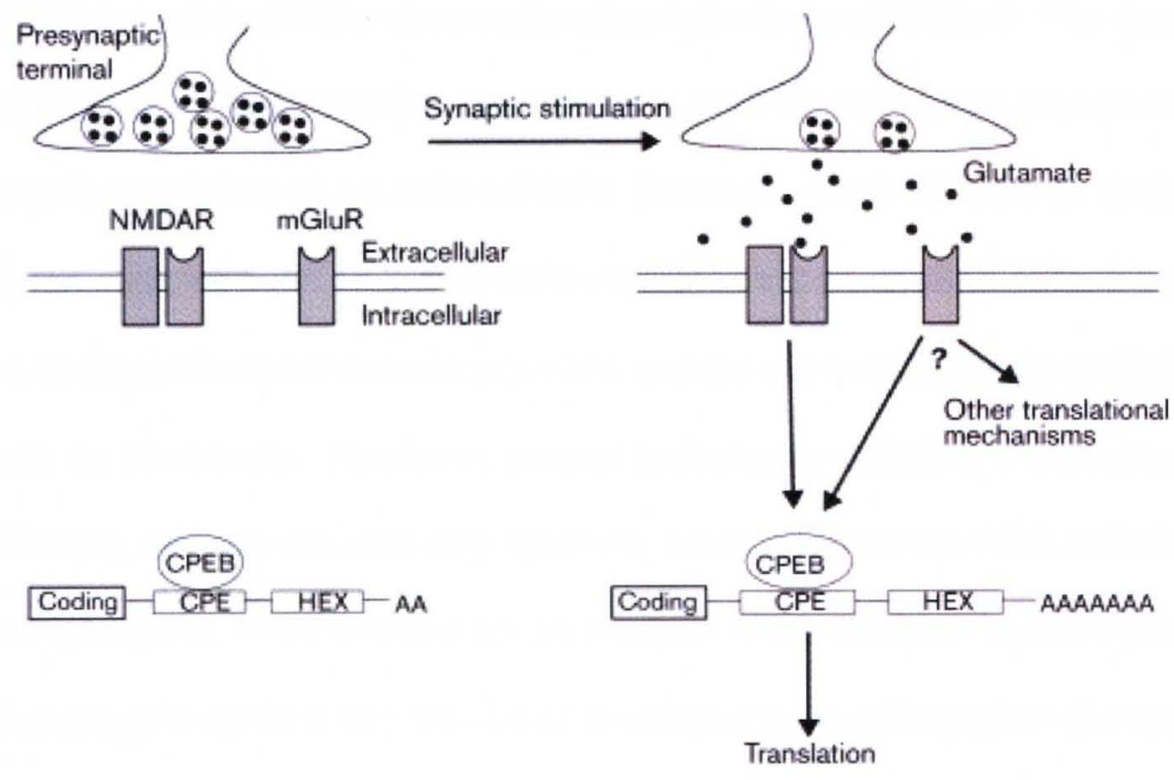


long-term memory and long-term synaptic plasticity. However, the mechanism by which translation is controlled and by which the proteins encoded modulate the prolonged changes biochemically is largely unknown. Some factors, including RNA-binding proteins and their target mRNAs at synaptic sites have been identified. For example, CPEB1 and Fragile-X Mental Retardation Proteins (FMRP) both have a function in the localization and translation of synaptic mRNAs. Because of the difficulty to manipulate primary neuron cultures such as low transfection rate for non-dividing cells, biochemical approaches to study the mechanisms are often carried out in more facile and efficient systems such as the oocyte. However, several techniques including the development of knockout animals, various viral delivery systems, small interference RNA (siRNA) knockdown techniques, have enabled some analysis of translational mechanisms in neurons. A surrogate system has also been employed by injecting brain-derived mRNAs into Xenopus oocytes to test their polyadenylation by CPEB1.

CPEB1 is localized to dendritic layers of the hippocampus, the synapses in cultured neurons, and PSDs in adult brain. CPEB1 was phosphorylated upon NMDAR receptor activation, and triggered the polyadenylation and translation of CaMKIla mRNA at the synaptic sites (Huang et al. 2002; Wu et al. 1998). In CPEB1 knockout mice, several forms of electrophysiological features of Schaffer CA1 neurons in response to single 100- $\mathrm{Hz}$ stimulation and single theta burst stimulation were impaired, suggesting CPEB1 plays a role in synaptic plasticity (Alarcon et al. 2004). Administration of CPEB siRNAs to the Aplysia neurons resulted in failure of the maintenance of long-term facilitation ( $\mathrm{Si}$ et al. 2003a).

Very few mRNAs are known CPEB1 targets in neurons. CaMKIla (Wu et al. 1998) and tPA (Shin et al. 2004) are two known mRNAs that undergo activity-dependent polyadenylation in hippocampal neurons. However, neither of these mRNAs appeared 
to be responsible for the deficiency in plasticity observed in CPEB1 knockout mice (Silva et al. 1992b; Frey et al. 1996). This suggested the presence of other mRNAs in neurons whose translation is regulated by CPEB1. A recent study screening mouse brain mRNAs using the surrogate system identified six mRNAs (all have potential involvement in synaptic plasticity): AMPA receptor binding protein (ABP), a potassium channel ELK2, CaMKIIס, microtubule-associated protein 2 (MAP2), and rat calmodulin (RCM3) mRNAs which became polyadenylated in response to synaptic activity (Du and Richter 2005). The same study demonstrated that in synaptosomes treated with glutamate, the proteins levels of MAP2 and ABP increased significantly by $35 \%$ and $72 \%$, respectively, compared to untreated control.

CPEB1 can be activated via several pathways. The induction of CPEB1 phosphorylation and polyadenylation in the hippocampus can be elicited by NMDA, but not AMPA (Huang et al. 2002). Kainate has been demonstrated to trigger CaMKIla up-regulation in the CA3 region of the hippocampus, an event that was blocked by a protein synthesis inhibitor cyclohexamide (Lee et al. 2003); kainate was also shown to stimulate polyadenylation (Du and Richter 2005). It is likely that kainate-induced translation of CaMKIla occurred through CPEB1-mediated polyadenylation, which would indicate that CPEB1 can be activated via kainate glutamate receptors. Another study observed that metabotropic glutamate activation triggered the polyadenylation of tPA mRNAs, suggesting that CPEB1 may be activated through metabotropic glutamate receptors (Shin et al. 2004).

2) CPEB2-4 and synaptic plasticity 
Although all four CPEBs have been identified in the nervous system, and the expression pattern has been characterized in some regions of the brain including the hippocampus, very little is known about the functions of CPEB2-4 in synaptic plasticity. CPEB3 is the second CPEB that has been under investigation in recent years. The mRNA of a key synaptic plasticity molecule, GluR2, can be bound by CPEB3. CPEB3 siRNA delivered to neurons led to elevated levels of GluR2 protein (Huang et al. 2006).

Although some aspects of the involvement of CPEB1 in synaptic plasticity have been revealed, such as the regulation of CaMKIla translation, little is known about the roles of CPEB2-4 in synaptic plasticity. Sequence alignment demonstrated a close proximity of CPEB2-4 in the family tree but a wide distance to CPEB1. CPEB2-4 lack the phosphorylation site for aurora kinase, a known activator for CPEB1 (Theis et al. 2003). But some alternatively spliced isoforms of CPEB2-4 have the B-region which is absent in CPEB1. The B-region possesses phosphorylation sites for PKA, CaMKIla, and p70S6 kinases (Theis et al. 2003). These data indicated that CPEB2-4 may be activated via distinct pathways than CPEB1. In kainate-induced seizures in the hippocampus, the expression of CPEB3 and CPEB4, but not CPEB1, were dramatically increased (Huang et al. 2006), suggesting that CPEB3 and 4 are responsible for different forms of plasticity compared to CPEB1. A U-rich loop secondary structure in the 3' UTR of mRNAs, but not CPE, is required for CPEB2-4 binding (Huang et al. 2006), indicating CPEB2-4 have discrete set of mRNA targets distinct from CPEB1. However, functional compensation or redundancy between CPEB1 and CPEB2-4 is not ruled out, since LTP was not completely eradicated in CPEB1 knockout mice. What is noteworthy is that CPEB3 may form a positive or negative feedback loop for CPEB1, since its mRNA contains two CPE sites in the 3' UTR. 


\section{CPEB and RNA localization}

CPEB regulated translation of CPE-containing mRNAs is a temporally or spatially tightly controlled process. It is the common underlying foundation for oogenesis, embryogenesis and would be a requirement for the demonstration of specificity of synaptic plasticity. Both CPEB protein and its target mRNAs need to be localized to the proper location for the local control of translation. The cis-element CPE in the 3' UTR of mRNA molecules and the RNA-binding protein CPEB have been demonstrated in the transport of such mRNAs.

1) CPEB and mRNA localization in Drosophila

Localized maternal mRNA is essential for the establishment of polarity in early development. The Drosophila CPEB homolog, orb, is required at multiple steps of oogenesis and is important for the establishment of both dorsal-ventral (DV) and anterior-posterior (AP) poles in early embryo development. Several maternal mRNAs are known to be involved in the establishment of these polarities, such as bicoid (St Johnston and Nusslein-Volhard 1992), nanos, oskar (Ephrussi et al 1991), fs(1)K10 and gurken (Haenlin et al. 1987, Cheung et al. 1992; Neuman-Silberberg and Schupbach 1993). Under normal development these mRNAs are localized to a specific pole and are translated upon fertilization to generate gradient proteins which then direct the localization or expression of other genes to generate the polarity of the developing embryo. Localization of such mRNAs to specific locations requires both cis-elements and trans-elements. The cis-elements are specific "tags" in the mRNA sequence that mark the mRNA for localization. They are specific motifs to be recognized and bound by 
RNA binding proteins. The trans-elements are RNA-binding proteins that transport and anchor the mRNA molecules to proper locations. Such trans-elements include general cytoskeleton proteins such as microtubules (Pokrywka and Stephenson 1991; Theurkauf et. al 1992) and staufen protein (Ferrandon et al. 1994; Micklem et al. 2000). The Drosophila CPEB homolog, orb, is involved in mRNA localization (Lantz 1992), with a complex localization pattern evident during oogenesis (Bally-Cuif et al. 1998). Several orb site mutations in Drosophila oocytes caused stalled development in the 8-cell cyst or 16-cell cyst stage. A less severe mutation did not stop the development but did cause abnormal distribution of mRNAs along both AP and DV poles. Maternal mRNAs such as oska, gurken and fs(1)K10 are misplaced in the orb mutant (Christerson and Mckearin 1994; Lantz et al. 1994).

2) CPEB and the mRNA transportation in Xenopus oocytes

During Xenopus oocyte maturation, mRNAs coding for c-mos, cdk2, several cyclins among others, undergo cytoplasmic polyadenylation and translational up-regulation. Several mRNAs are regionally concentrated in Xenopus oocyte (Schnapp et al., 1997). CPEB proteins and maskin are moderately concentrated in the animal pole in Xenopus oocytes, and became more concentrated in eggs and early embryos due to the dominant protein degradation in the vegetal pole. Both proteins colocalized with a-tubulin on the centrosomes and mitotic spindles in the animal pole. Two mRNAs, xub3 and cyclin B1, both containing CPE motifs in the 3' UTR, are localized with or near CPEB on centrosomes or spindles (Groinsman et al. 2000). While the importance of xbub3 is not known, cyclin B1 is important for cell cycle. Injection of CPEB antibody, mutant CPEB with disrupted phosphorylation site, or a polyadenylation inhibitor cordycepin (3'-dATP), 
blocked cytoplasmic polyadenylation-induced translation of cyclin B1 (Groinsman et al. 2000). A CPEB mutation that does not bind to the centromere in vivo disrupted the localization of cyclin B1 mRNA. Such findings indicated a role of CPEB for the localization of CPE-containing mRNAs, consistent with the observation in Drosophila and zebrafish.

3) CPEB and mRNA localization in the CNS

In neurons, local translational regulation of synaptically enriched mRNAs such as the activity-dependent cytoskeleton protein Arc (Steward et al. 1998), MAP2, CaMKIla (Roberts et al. 1998), BDNF, and TrkB (Tongiorgi et al. 1996) is implicated in synaptic plasticity. The mechanism for the localization of such mRNAs to synapses is yet to be delineated. Recent evidence indicates that the cis-element CPE is sufficient to direct CPE-containing mRNAs to the dendrites, and the process of such localization is facilitated by CPEB. CPEB, together with its adaptor protein maskin, forms RNAcontaining particles that were transported to dendrites in a microtubule-dependent manner, suggesting that these mRNAs were transported in a translationally dormant form. Such transportation particles also contained the molecular motor dynein and kinesin. The transport of dendritically localized mRNAs was enhanced in neurons overexpressing CPEB, but inhibited in neurons expressing mutated CPEB or in CPEB knockout mice (Huang et al. 2003).

5. Synaptic plasticity in the neural retina 
The neural retina is located at the back of the eye (figure 14). It receives light stimulus and sends the signal to the upper levels of the visual system. The retinal circuitry is composed of primarily vertical pathways with inhibitory modulations from lateral pathways (figure 15): Light projects on the light-sensitive photoreceptor cells in the outer nuclear layer (ONL), which will transmit signal to the interneurons- bipolar cells in the inner nuclear layer (INL), which will then relay signal to the ganglion cells in the retinal ganglion cell layer (RGC). Retinal ganglion cells ultimately collect the signal and project it to higher center of the visual system. The excitatory neurotransmitter glutamate is the predominant neurotransmitter employed in the vertical pathway.

In addition to the vertical pathway, there are two tiers of lateral pathways: Horizontal cells in the outer boundary of the INL integrate signals from multiple photoreceptor cells and modulate them as necessary before sending them on to bipolar cells. Amacrine cells in the inner boundary of the INL integrate and modulate signals from bipolar cells before sending them on to the retinal ganglion cells. The predominant neurotransmitter used in the lateral pathways is gamma-aminobutyric acid (GABA).

Synaptic connections of the retina are located within well-characterized laminar structures: the outer plexiform layer (OPL) and the inner plexiform layer (IPL). The OPL is composed of synaptic connections between photoreceptors, bipolar cells and horizontal cells. The IPL is composed of synaptic connections between bipolar cells, ganglion cells and amacrine cells.

Synaptic plasticity has long been recognized at higher levels of CNS, such as the cerebral cortex (Cheetham et al. 2008), the hippocampus (Nicoll and Schmitz 2005), and the cerebellum (Jorntell and Hansel 2006). It has also been depicted at higher levels of the visual system (Karmarkar and Dan 2006). Neuroscientists have long thought that 
Figure 14. Diagram of the eye (www.nei.nih.gov/health/eyediagram/eyeimages2.asp)

The diagram shows the location of the retina within the eye. The retina is the innermost layer at the back of the eye. Light enters the cornea, passes through the lens and is projected onto the retina, where it is converted into electrochemical signals and sent through the optic nerve to higher centers in the brain for further process of visual perception. 
Figure 14

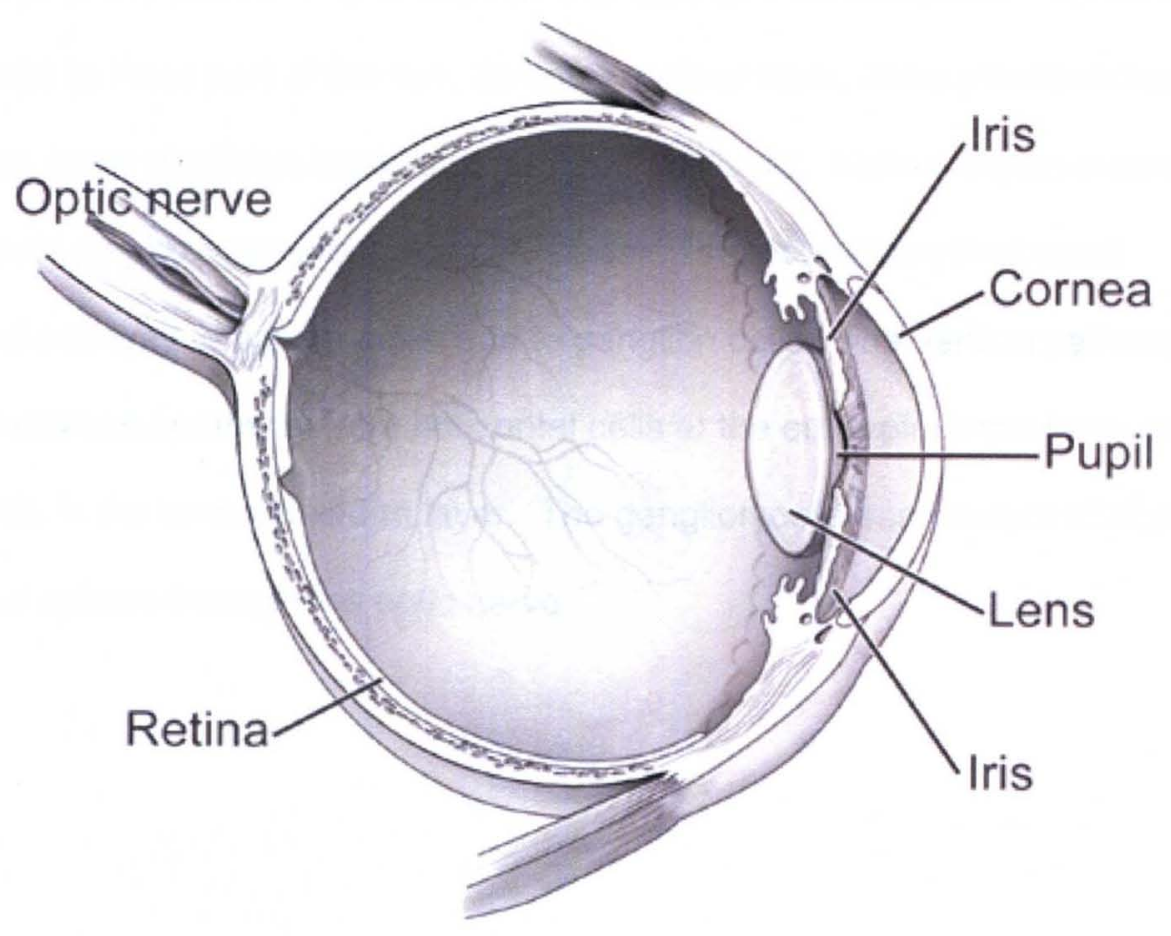


Figure 15. Diagram of the retina (Kandel et al. 2000)

This figure illustrates the laminar structure, the major cell types, and the vertical and horizontal pathways of the retina. The retina has a unique laminar structure. From the outer part of the eye to inner part of the eye, lie outer nuclear layer, outer plexiform layer, inner nuclear layer, inner plexiform layer, and ganglion cell layer. Light stimulus enters the eye and projects on photoreceptors (rods or cones). Photoreceptors then send signals via vertical pathway to bipolar cells, and to ganglion cells. The vertical pathway is modulated by horizontal pathway from horizontal cells at the outer plexiform layer and from amacrine cells in the inner plexiform layer. The ganglion cells send output to higher levels of the visual system through the optic nerve. 
Figure 15

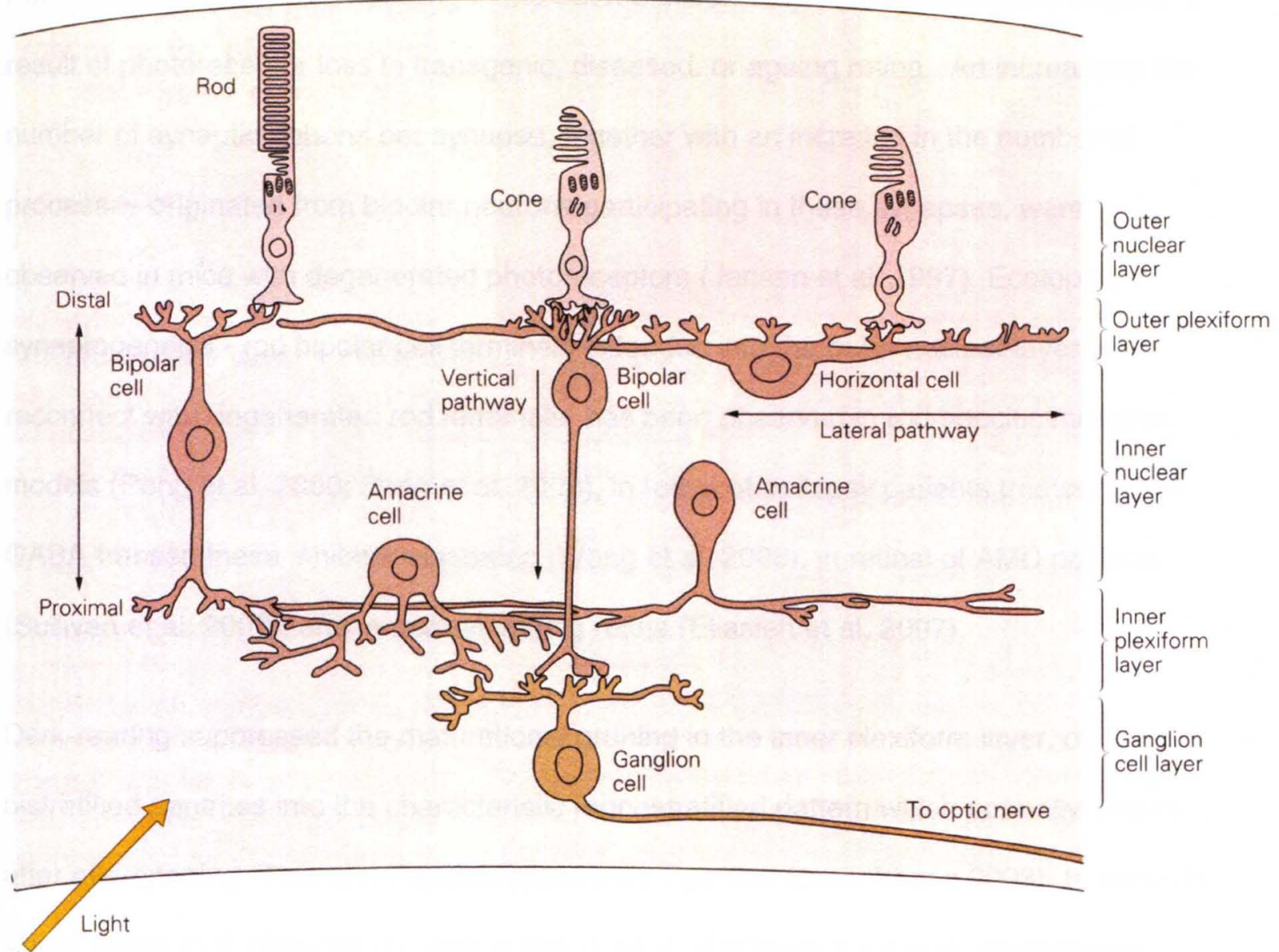


the neural retina lacks the property of plasticity. However, recent studies have provided several lines of evidence, including morphological, molecular and electrophysiological observations, which support the notion that synaptic plasticity is present in the retina.

For example, synaptic remodeling in the outer plexiform layer has been observed as a result of photoreceptor loss in transgenic, diseased, or ageing retina. An increase in the number of synaptic ribbons per synapse, together with an increase in the number of processes originated from bipolar neurons participating in these synapses, were observed in mice with degenerated photoreceptors (Jansen et al. 1997). Ecotopic synaptogenesis - rod bipolar cell terminals extended into the outer nuclear layer to reconnect with degenerated rod terminals- has been observed in rod-specific mutation models (Peng et al. 2000; Peng et al. 2003), in retina of epilepsy patients treated with a GABA-transaminase inhibitor vigabatrin (Wang et al. 2008), in retinal of AMD patients (Sullivan et al. 2007), and in normal ageing retina (Eliasieh et al. 2007).

Dark-rearing suppressed the maturational pruning in the inner plexiform layer, of bistratified dentrites into the characteristic monostratified pattern which normally occurs after eye-opening (Tian and Copenhagen 2001; Tian and Copenhagen 2003). In addition, visual deprivation elevated the expression of several important synaptic components, such as CaMKII, GluR1 and NR1 in the retinal ganglion cells (Xue et al. 2001; Xue and Cooper 2001). Light responsiveness measured as electroretinogram (ERG) and oscillatory potentials (OP) were inhibited in both young and adult dark-reared animals (Vistamehr and Tian 2004). Even under normal diurnal environment, AMPARs undergo rapid recycling via endocytosis and exocytosis in the retinal ganglion neurons (Xia et al. 2006), and such rapid cycling is primarily restricted to GluR2-containing AMPARs (Xia et al. 2007), which play a pivotal role in long-term synaptic plasticity (Tanaka et al. 2000; Isaac et al. 2007). Together, these data support the notion that plasticity is a 
phenomenon that is a characteristic of retinal synapses as it is for synapses in other parts of the CNS.

Synaptogenesis is a postnatally ongoing process in the rodent retina. Rodent retina is premature and the synaptic structure is not well-established at the time of birth. It is not until approximately eye-opening stage, which is about 14 days postnatally in mouse that the synaptic structure in the plexiform layers becomes functional (Tian and Copenhagen 2001). Synaptogenesis in the inner plexiform layer (IPL) starts at day 3 and continues until before eye-opening, when the rate of synaptogenesis in the INL drops precipitously (Fisher 1979b; Fisher 1979a). Robust glutamatergic synaptic formation occurs in the second postanatal week, and synaptic sublamina becomes visible in the inner plexiform layer on postnatal day 12 (P12) (Sherry et al. 2003). Synaptic connections in OPL begin to develop on postnatal day 3 (P3) and are well established by P12, when the first light response was recorded as ERG (Rich et al. 1997). A diagram illustrating the morphological maturation of synaptic structures between different types of cells is presented in figure 16.

\section{CPEBs and CPE-containing mRNAs in the retina}

Several findings have indicated the presence of cytoplasmic polyadenylation in the retina.

Of the four mouse CPEBs (CPEB1-4) characterized to date (Gebauer and Richter 1996; Theis et al. 2003; Kurihara et al. 2003), one (CPEB1) has been shown to be present in the mouse retina of both wildtype and rod-ablated model (rdta) mice (Liu et al. 2000). Multiple studies have shown that the mRNA of a key molecule for synaptic plasticity, CaMKIla, which has two CPE motifs in its 3' UTR, is present in the retina (Liu and Cooper 1996; Cooper et al. 1995; Terashima et al. 1994), and could possibly be involved 
Figure 16. Timeframe of synaptogenesis in mouse retina (modified from Olney 1968) Time table of morphological maturation for the synapses in mouse retina. Heavy lines indicated periods of accelerated development; broken lines indicated periods of relative quiescence or slow development. R-photoreceptors; H-horizontal cells; BP-bipolar cells; A-amacrine cell; G-ganglion cell. 
Figure 16

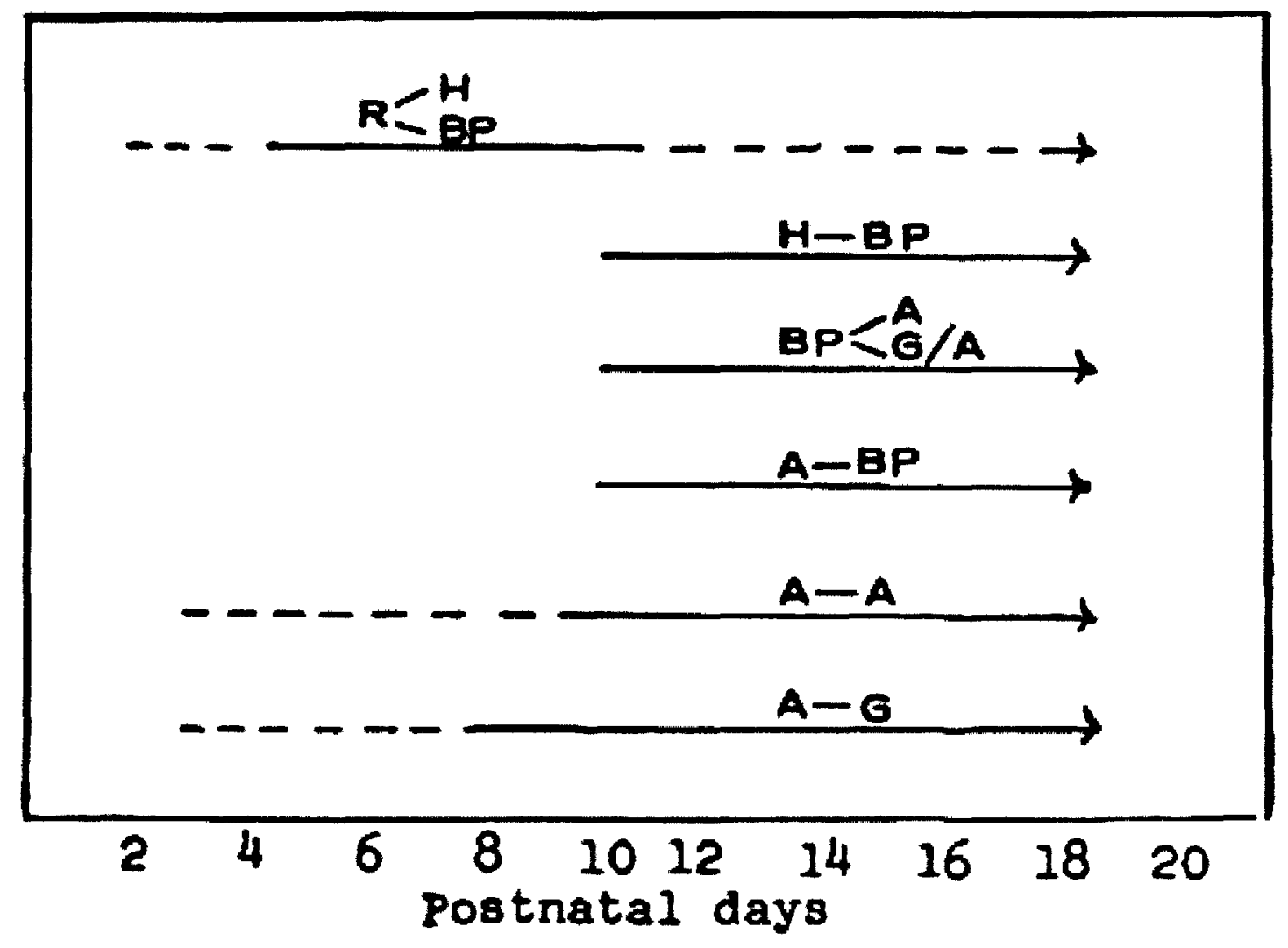


in the dendritic development and/or plasticity (Okada et al. 1999; Weiler et al. 1996; Weiler et al. 1995). The early growth response factor-1 (EGR1), a member of zinc-finger transcription factor family, is expressed in synapses across the retina. The longer form of its two alternative spliced mRNA isoforms possesses a CPE in its 3' UTR, and therefore can translate rapidly upon excitatory stimuli such as light and NMDAR activation (Simon et al. 2004). A key synaptic plasticity molecule GluR2 contributes to the physiological property of AMPARs and is present in neurons in the retina (Hughes et al. 1992; Hamassaki-Britto et al. 1993). It is unknown whether the other CPEBs are also expressed in the retina and this lack of knowledge will hamper further characterization of any putative retinal synaptic plasticity.

In this study, the presence, the localization and the expression patterns of all four CPEBs in the mouse retina were investigated. The developmental appearance of all four CPEBs during postnatal development was characterized. Potential mRNA targets for CPEB1, whose cis-acting element is well established, were studied. The alternative spliced isoforms of CPEB3 associated with the retina was explored. 


\section{CHAPTER II \\ HYPOTHESIS AND SPECIFIC AIMS}

The retina is a favorite model for the study of the central nervous systems because it is an accessible part of the brain (Dowling 1987) with an endowed fine laminar structure and easy access for molecular and electrophysiological manipulations. The well-defined cellular types and neuronal pathways between distinct cell types could make the retina an invaluable tool for neurobiological studies.

Synaptic plasticity has long been appreciated at higher levels of the visual system. The neural retina as part of the central nervous system contains the same kind of synaptic machinery as other regions of the brain (Dowling 1987). This prompted the questions regarding the presence of synaptic plasticity in the retina. Several lines of evidence demonstrated that the retina is capable of remodeling its synaptic structures. The synaptic structures of the IPL of mouse retina are disoriented at birth, but become wellestablished multiple sublaminae before eye-opening. Such a process was retarded in animals subjected to dark-rearing (Tian and Copenhagen 2001). The fine structures of the OPL synaptic connections have also been demonstrated to undergo profound remodeling under certain conditions. Morphological alterations have been observed in rod bipolar cell terminals in ageing or diseased models. Light responsiveness measured as ERGs and OPs were inhibited in both young and adult dark-reared animals (Vistamehr and Tian 2004). The NMDAR-associated currents were down-regulated in 
concurrence with eye-opening in naïve animal, but such an alteration was impeded in dark-reared animals. These manifestations in morphology and electrophysiology indicated that the retina has some degree of plasticity.

Some key molecules involved in activity-dependent synaptic plasticity have been identified in the retina. Cytoplasmic polyadenylation, the underlying mechanism for activity-dependent long-term plasticity in the hippocampus and the visual cortex, has been indicated to be present in the retina. A previous study observed CPEB1 in the retina of both wildtype mice and rdta mice (Liu et al. 2000). Other molecules implicated in synaptic plasticity such as CaMKIla and GluR2 mRNA, which can be recognized by CPEB1 and CPEB3 respectively, are enriched in the synapses of the retina. EGR1, an early gene that encode for a transcriptional factor in long-term synaptic plasticity, is present in the retina with two alternative splicing isoforms. The translation of the longer version of EGR1 transcript which has CPE in its 3' UTR is regulated by excitatory stimuli such as light and NMDAR activation in the retina (Simon et al. 2004). If the elements which endow synapses with the property of plasticity can be found in the retina, it would enhance its reputation as a more accessible model for such studies.

Based on the observations of previous studies, I hypothesize that more than one paralog of CPEB is present in the retina.

Aim1: To determine the expression pattern of CPEB1 in the retina.

Specifically, this aim will investigate the types of cells expressing CPEB1; the regulation of CPEB1 during the development, and the potential mRNA targets of CPEB1 in the retina. The localization of CPEB1 will be explored with in situ hybridization at the mRNA level and immunofluorescence at the protein level. The temporal appearance of CPEB1 
during post-natal development will be assessed with the aid of real-time PCR for mRNA and with Western blots and immunocytochemistry for protein. The potential targets of CPEB1 will be identified from nucleotide and protein databases using a bioinformatics tool in combination with rapid amplification of cDNA ends (RACE)-PCR. Several candidates will be assayed with the aid of the polyadenylaiton test (PAT) to demonstrate their status of polyadenylation in the paradigms of a light-dark period as well as during postnatal development.

Aim 2: To characterize the expression of CPEB3 in the retina.

Specifically, this aim will explore isoforms of CPEB3 mRNA and protein; the types of cells that express CPEB3, and the regulation of CPEB3 during postnatal development. Bioinformatic analysis will be used to identify current known CPEB3 transcript variants and protein isoforms. RT-PCR will be used to characterize the tissue distribution of each isoform in the retina and in multiple other tissues. In situ hybridization and immunofluorescence microscopy will be used to determine the localization of CPEB3 mRNA and proteins, respectively. Real-time PCR and Western blots will be used to investigate the regulation of CPEB3 transcripts and proteins during post-natal development.

Aim 3: To compare CPEB1-4 in the retina.

Specifically, this aim will investigate the types of cells that express mRNAs for CPEB1-4; the temporal appearance of CPEB1-4 during postnatal development, and the relative expression levels of CPEB1-4 in the retina. Comparison of the localization of four 
CPEBs in the retina will be performed with the aid of in situ hybridization. Comparison of the appearance of all four CPEBs during post-natal development will be performed with the aid of real-time PCR. Cross-comparison quantification with the aid of real-time PCR data will be used to assess the relative abundance of CPEB1-4. 


\section{CHAPTER III}

\section{MATERIALS AND METHODS}

1. Animal handling and tissue preparation

All animal experiments were carried out in compliance with the National Institutes of Health Guide for the Care and Use of Laboratory Animals, the University of Louisville Institutional Animal Care and Use Committee (IACUC) animal care regulations, as well as the Association for Research in Vision and Ophthalmology (ARVO) statement for the use of animals in ophthalmic and vision research.

C57/BL6 mice were used for this study (Charles River Laboratories, Davis, CA). Mice at the age of postnatal day $1,7,12,14,16,30$, and 60 were used for developmental studies. All the pups younger than 21 days old were raised together with their lactating mothers. All animals were housed in normal diurnal cycles of 12-hour light/12-hour dark unless otherwisely specified. Each experimental group was composed of at least 5-6 animals.

For tissue collection, mice were euthanized with $\mathrm{CO}_{2}$ followed by cervical dislocation. For RNA and protein extraction, the eyes were removed; retinas were quickly dissected and immediately frozen on dry ice before proceeding for extraction. Similar procedures were followed when other tissues were collected for RNA extraction. For in situ 
hybridization and immunohistochemistry, the eyes were removed and immediately fixed in $4 \%$ paraformaldehyde for 12 hours at $4^{\circ} \mathrm{C}$. The eyes were then transferred to PBS buffer with $30 \%$ sucrose for dehydration for two weeks at $4{ }^{\circ} \mathrm{C}$. Snap-freezing technique was used for the embedment: A stainless isopentane container was placed on dry ice. Immediately before embedding, crushed dry ice was scattered in the isopentane until no bubbles were generated from the mixture, an indication that the temperature reached below $-70^{\circ} \mathrm{C}$. The retina placed in tissue mounting medium in a mold was then floated on the fluid (by holding the mold with a pair of forceps). Make sure that the mold was not submerged in the isopentane/dry ice mixture. The embedding process should take several seconds. The mounted tissue was then cut on a cryostat the same day or frozen air-tight at $-80^{\circ} \mathrm{C}$ for future processing.

\section{Bioinformatic analysis - mRNA targets for CPEB1}

In order to identify additional mRNAs potentially regulated by CPEB1, a bioinformatics program, "CPE Motif Detector" was developed in collaboration with Dr. Eric Rouchka. This program took into account the CPE motif, the presence of poly(A) signal sequence, and a distance measure (spacer nucleotides) between those two. A database of 3' UTR sequences was downloaded from UTResource (www.ba.itb.cnr.it/UTR/) and these $3^{\prime}$ UTRs were searched. Initial screens of CPE elements were found by looking for the pattern TTTTTATT upstream of the poly-A signal AATAAA with a spacer sequence between the two. For instance, if the expected spacer is between 50 and 100 bases, the sequence can be searched using the Perl regular expression:

$\$$ seq $=\sim$ "ttttatt(a|t|c|g|n)\{50,100\}aataaa") 
In order to search for two or more CPE sites, the CPE motif was expanded to search for:

\$seq = "ttttt(a|g)tt(a|t|c|g|n)\{1,\}tttt(a|g)tt(a|t|c|g|n)\{1,\}aataaa"

This pattern will allow alternative CPE motifs. Note, however, that this pattern does not limit the range of the spacer between the two motifs, or between the second motif and the poly-A signal.

\section{Data mining -isoforms of CPEB3}

UniGene database from NCBI databases (http://www.ncbi.nlm.nih.gov) was used for the identification of CPEB3 transcript variants. $\mathrm{NCBI}$ and Swiss-Prot/TrEMBL (http://expasy.org/sprot/) databases were used for the identification of CPEB3 protein isoforms. Sequence alignments of transcripts and of proteins were carried out using ClustalW 2.0.5 (http://www.ebi.ac.uk/Tools/clustalw2/); sequence alignments of transcripts with proteins were carried out using Vector NTI (Invitrogen, Carlsbad, CA); sequence alignments of CDNAs with genomic DNA was carried out using UCSC mouse genome Blat (http://genome.ucsc.edu).

\section{Isolation of RNAs}

Frozen retina was homogenized rapidly using PowerGen 250 homogenizer (Fisher Scientific, Pittsburgh, PA). Total RNA was extracted using RNeasy mini kit (Qiagen, Valencia, CA) following the manufacturer's instructions. Briefly, tissue was homogenized for 10 seconds in an appropriate volume of lysis buffer, followed by chilling 
on ice. This was repeated twice at 10 seconds each time. The lysate was then centrifuged at $14,000 \mathrm{xg}$, and the supernatant was collected and transferred to a new tube. One volume of $70 \%$ ethanol was added and the mixture was quickly loaded to RNeasy spin columns and centrifuged briefly. The columns were then washed with buffer RW1 once, with buffer RPE twice, before RNA was eluted in RNase-free $\mathrm{H}_{2} \mathrm{O}$.

RNA concentration was determined using a BioPhotometer (Eppendorf, Westbury, NY). The concentration of RNA was adjusted by dilution to an A260 within the range 0.1-1.0. The concentrations of RNA samples were calculated based on the absorbance at $260 \mathrm{~nm}$ using the following equation:

RNA concentration $(\mu \mathrm{g} / \mathrm{ml})=\mathrm{A} 260 \times 40 \times$ dilution factor

$5 \mu$ of each RNA sample was electrophoresed on an agarose gel. The quality of RNA was determined from the ratio of $28 \mathrm{~S} / 18 \mathrm{~S}$ on agarose gel. RNA was frozen in $-80^{\circ} \mathrm{C}$ for long term storage.

\section{Regular RT-PCR}

$0.2 \mu \mathrm{g}$ of total RNA was used for every $20 \mu \mathrm{l}$ RT reactions. Omniscript reverse transcriptase (Qiagen, Valencia, CA) and oligo-dT 15-mers were used to enable efficient and sensitive reverse transcription. Components were assembled using conditions according to the manufacturer's suggestions; reactions were incubated for $60 \mathrm{~min}$ at $37^{\circ} \mathrm{C}$.

$1 \mu \mathrm{l}$ of the cDNA was used for subsequent PCR. HotStarTaq DNA polymerase (Qiagen, Valencia, CA) was used for robust and specific amplification. Components were 
assembled according to manufacturer's instructions. The following settings of the thermocycler were used:

Initial activation step: $95^{\circ} \mathrm{C}, 15 \mathrm{~min}$.

Amplification:

40 cycles of: $94^{\circ} \mathrm{C}, 30 \mathrm{sec}$;

$55-60^{\circ} \mathrm{C}$ (approximately $5^{\circ} \mathrm{C}$ below $\mathrm{Tm}$ of primers), $30 \mathrm{sec}$; $72^{\circ} \mathrm{C}, 1 \mathrm{~min}$.

Final extension: $\quad 72^{\circ} \mathrm{C}, 10 \mathrm{~min}$.

Exon-exon spanning, gene-specific primers (IDT, Coralville, IA) were used for the identification of CPEB1-4 and the positive control $\beta$-actin transcripts (table 3). Exonexon spanning, transcript specific primers were used for the identification of CPEB3 alternatively spliced variants (table 4). The Resultant PCR products were visualized on $1 \%$ agarose gel and photographed. Each specific band was purified from the gel and sequenced for confirmation of its identity.

\section{3' RACE RT-PCR}

Total RNA from mouse retina was used for 3' RACE RT-PCR. FirstChoice RLM-RACE kit (Ambion, Austin, TX) was used for the amplification. An overview of the procedure is illustrated in figure 17. Briefly, first strand cDNA was synthesized from the retinal RNA using the 3' RACE Adapter primer - an oligo-dT primer with an anchor sequence at the 5' end and degenerations at the 3' end (table 5). Specifically, degenerative oligo-dT primers with an $\mathrm{N}$ and a $\mathrm{V}$ at the $3^{\prime}$ end ( $\mathrm{V}$ represents any of $\mathrm{A}, \mathrm{C}$, or $\mathrm{G}$, while $\mathrm{N}$ 
Table 3. Primer sets used for CPEB1-4 and $\beta$-actin in regular RT-PCR

\begin{tabular}{ccc}
\hline Gene & Primer & Sequence \\
\hline 3-actin & fwd & 5'-TGAAGATCAAGATCATTGCTCC-3' \\
& rev & 5'-TTAAAAAAACAAAGCCATGCC-3' \\
\hline CPEB1 & fwd & 5'-CACCTTCCGTGTTTTTGGC-3' \\
& rev & 5'-ATCCCAGCATACACCACTCC-3' \\
\hline CPEB2 & fwd & 5'-CTCTCAAGGGTCGTCTGAGC-3' \\
& rev & 5'-AATGCATAGCCTTTTGGTGG-3' \\
\hline CPEB3 & fwd & 5'-CATCAAGGATAAACCGGTGC-3' \\
& rev & 5'-GAAGAATGGGGCAAACTTCC-3' \\
\hline CPEB4 & fwd & 5'-ATTTTTCCTTTTCCGGAACG-3' \\
& rev & 5'-GTATCGTTCCACTCTCTCCCC-3' \\
\hline
\end{tabular}


Table 4. Primers used to identify the alternatively spliced transcripts of CPEB3 in regular RT-PCR

\begin{tabular}{|c|c|c|}
\hline Gene & Primer name & Primer sequence \\
\hline \multirow[t]{13}{*}{ CPEB3 } & $\# 1$ & 5'-TGTCAATGTCGTTGTGTTGAAGTTGC-3' \\
\hline & \#3 & 5'-GACTCCCACACCACAAGGATACACA-3' \\
\hline & \#4 & 5'-GAAGGCGTCTTCAAAGGGAAAGAGA-3' \\
\hline & \#5 & 5'-TATGATAAGGACTGACCATGAGCCTCTG-3' \\
\hline & \#7 & 5'-AGGAGCTATGGGCGGAGACGA-3' \\
\hline & \#8 & 5'-GCTGAGTCCCCAATGCCTTAGC-3' \\
\hline & $\# 13$ & 5'-CCCGTTTGTCAATGTCGTTGTGTT-3' \\
\hline & \#16 & 5'-AAGGATAAACCGTTGAACTGGCA-3' \\
\hline & $\# 17$ & 5'-CATGAGCCTCTGAAAGGTAAACACT-3' \\
\hline & \#18 & 5'-AAGACCGACCTCGTCTCCGC-3' \\
\hline & $\# 19$ & 5'-CATGAGCCTCTGAAAGGACGC-3' \\
\hline & \#20 & 5'-GAAGACCGACTGTTAAGTGCCATA-3' \\
\hline & \#21 & 5'-TTCGAGCTGTTGAACTGGCA-3' \\
\hline
\end{tabular}


Figure 17. Diagram of $3^{\prime}$ RACE

3' RACE (rapid amplification of cDNA ends)-PCR were used for the identification of CPE, PAS-containing mRNAs from the mouse retina.

Oligo(dT) primers with anchor sequences attached to the 5 ' ends were used for the reverse transcription. The resulted cDNA pool has a ubiquitous anchor sequence at the 3' end.

Nested PCR were used for the amplification. For the first round, forward primers containing CPE consensus sequence paired with reverse primers - 3' outer primers (part of the anchor region) were used. For the second round, forward primers containing PAS consensus sequence paired with reverse primers $-3^{\prime}$ inner primer (part of the anchor region) were used. For both PCR reactions, the first 3-5 rounds were carried out with annealing temperature at $37^{\circ} \mathrm{C}$. The annealing temperature was raised to $60^{\circ} \mathrm{C}$ after that. 
Figure 17

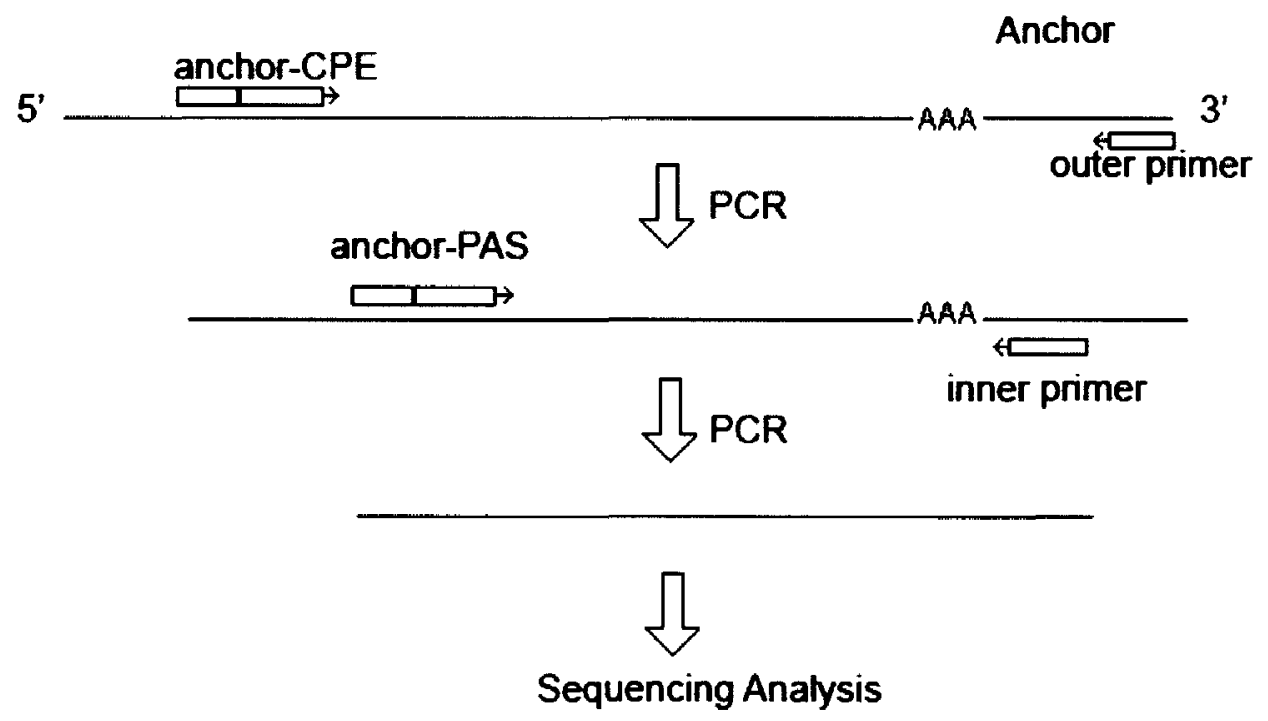


Table 5. Primers used for 3' RACE

\begin{tabular}{ll}
\hline \multicolumn{1}{c}{ Primer } & \multicolumn{1}{c}{ Sequence } \\
\hline 3' RACE Adapter Primer & 5'GCGAGCACAGAATTAATACGACTCACTATAGGT12VN3' \\
3' RACE Outer Primer & 5'GCGAGCACAGAATTAATACGACT3' \\
3' RACE Inner Primer & 5'CGCGGATCCGAATTAATACGACTCACTATAGG3' \\
wobbled CPE & 5'N14AAAATA3' \\
wobbled PAS & 5'N14TTTATT3' \\
\hline
\end{tabular}

Note: The first three primers were from FirstChoice RLM-RACE kit (Ambion). It has an anchor at the 5' end, as well as degeneration at the 3 ' end. $V$ represents any of $A, C, G$; whereas $\mathrm{N}$ represent any of $\mathrm{A}, \mathrm{C}, \mathrm{T}$, and $\mathrm{G}$. 
represents any of $A, T, C$, or $G$ ) make the annealing specifically occur where the poly $(A)$ starts, instead of randomly priming along the poly $(\mathrm{A})$ tail. This primer helps to generate a homogeneous 3' end (with respect to the length of the poly $(A)$ tail) for the first strand cDNA. M-MLV reverse transcriptase was used for reverse transcription and the reactons were incubated at $40^{\circ} \mathrm{C}$ for 1 hour.

The cDNA was then used in two steps of nested PCR reactions: For the first PCR, 3' RACE outer primer and a wobbled CPE primer were used; for the second PCR, 3' RACE inner primer and a wobbled PAS primer were used. The $3^{\prime}$ outer primer and the $3^{\prime}$ inner primer were complementary to the outer portion and inner portion of the 3' RACE Adapter primer, respectively (table 5). The wobbled CPE primer and the wobbled PAS primer have a random 14-mers in their 5' end, followed by sequences complementary to CPE and PAS motifs, separately. A thermostable DNA polymerase was used for the nested PCR. The following settings were used for the thermocycler:

Initial activation: $\quad 94^{\circ} \mathrm{C}, 3 \mathrm{~min}$ Amplification:

3 cycles of: $\quad 94^{\circ} \mathrm{C}, 30 \mathrm{sec}$;

$37^{\circ} \mathrm{C}, 30 \mathrm{sec}$

$72^{\circ} \mathrm{C}, 30$ sec.

35 cycle of: $\quad 94^{\circ} \mathrm{C}, 30 \mathrm{sec}$;

$60^{\circ} \mathrm{C}, 30 \mathrm{sec} ;$

$72^{\circ} \mathrm{C}, 30$ sec.

Final extension: $\quad 72^{\circ} \mathrm{C}, 30 \mathrm{sec}$

Because the wobbled CPE and the wobbled PAS have only 6-nucleotide match, the first three cycles of amplification was performed at a lower annealing temperature $\left(37^{\circ} \mathrm{C}\right)$ to 
ensure the sensitivity; the subsequent amplifications were performed at an annealing temperature of $60^{\circ} \mathrm{C}$ to ensure specificity.

The PCR products were loaded to agarose gel for electrophoresis. The separated bands were cut out, purified, and sequenced to identify their identity.

\section{LM-PAT assay}

Several methods to determine the length of poly $(A)$ tails of mRNA are illustrated in figure 18. Since the amount of RNA from mouse retinas was limited, we avoided the methods involving Northern blots. We used the ligation-mediated polyadenylation test (LM-PAT) assay for our study, which is briefly described below.

For ligation, $\sim 1 \mu \mathrm{g}$ RNA was mixed with 5'-phophor-(dT)15-mer and denatured at $65^{\circ} \mathrm{C}$ for $5 \mathrm{~min}$, then transferred to $42^{\circ} \mathrm{C}$ without ice-quenching step. A mix of T4 ligase (Promega, Madison, WI) with SuperScript II buffer, dNTPs and ATPs were prewarmed at $42^{\circ} \mathrm{C}$ then added to the RNA-primer mix, and incubated at $42^{\circ} \mathrm{C}$ for $30 \mathrm{~min}$. At the end of incubation, appropriate amount of oligo(dT)-anchor primers was added and the tube was transferred to $16^{\circ} \mathrm{C}$ for 2 hours.

For reverse transcription, the tube was transferred to $42^{\circ} \mathrm{C}$ for $2 \mathrm{~min}$. SuperScript II RNase $\mathrm{H}$ - reverse transcriptase (Invitrogen, Carlsbad, CA) was added and incubated at $42^{\circ} \mathrm{C}$ for 1 hour. Afterward, ligase and reverse transcriptase were inactivated by incubating at $65^{\circ} \mathrm{C}$ for $20 \mathrm{~min}$.

PCR was performed using HotStarTaq (Qiagen, Valencia, CA). The cDNA from the above ligation and RT reactions, gene-specific primers, and anchor primers (table 6) 
Figure 18. Determination of poly(A) length (Sallés et al. 1999)

a) Northern blot. The RNA molecule with longer poly(A) tail migrates at a slower rate.

b) Oligo(dT)/RNase H Northern. Poly(A) tails are annealed to Oligo(dT) before RNase $\mathrm{H}$ treatment, which removes RNA:DNA hybrid - the complete poly(A). RNase $\mathrm{H}$ treated samples are compared to untreated samples in the subsequent Northern blot. An approximate length of poly $(A)$ tail can be inferred with this method.

c) Rapid amplification of cDNA ends-PAT (RACE-PAT): Oligo(dT) primers with anchors sequence attached to the $5^{\prime}$ ends are used for reverse transcription reaction. A gene-specific primer and an anchor primer are used for the following PCR reactions. The mRNA with a longer poly $(A)$ tail will generate a longer smear (a mixture of amplicon with partial or full-length poly(A) tail).

d) Ligation mediated PAT (LM-PAT): Oligo(dT) primers and oligo(dT) primers with anchor sequences attached to the 5' ends are used. A ligation step is performed before the reverse transcription reaction to ligate these oligo(dT) together. $A$ gene-specific primer and an anchor primer are used for the subsequent PCR reactions. The amplicons generated using this method is less heterogeneous compared to $\mathrm{c}$ ). The position of the smear on the PCR gel reflect the relative length of the poly $(A)$ tails. 
Figure 18

Northern

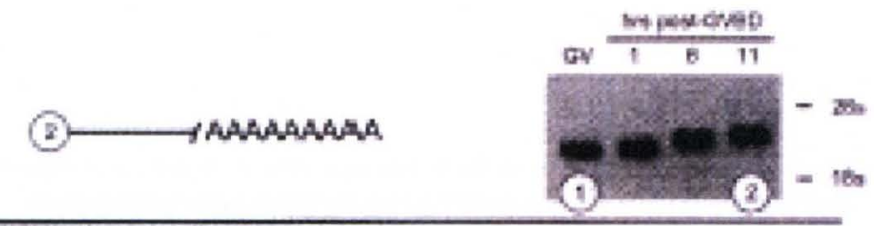

Oligo(dT)/RNase H Northern

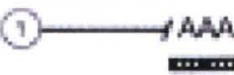

(2) AWALA WAAA

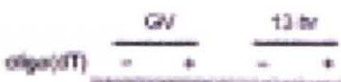

b
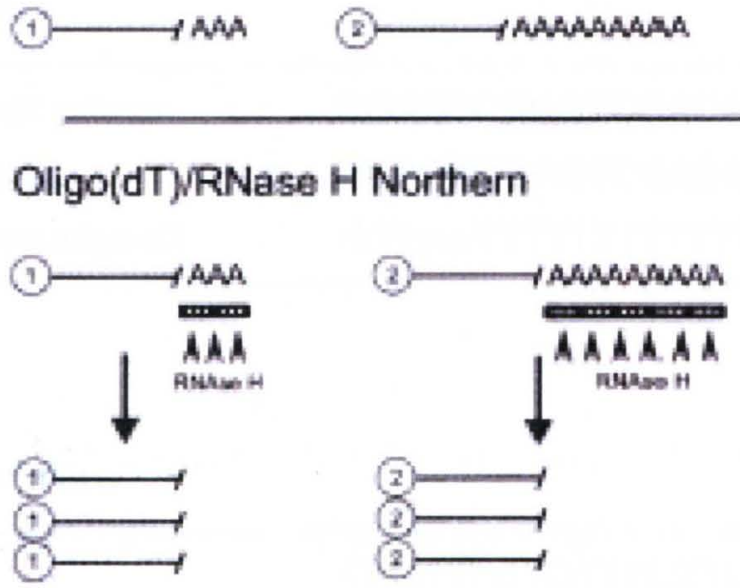

Pothen

wont
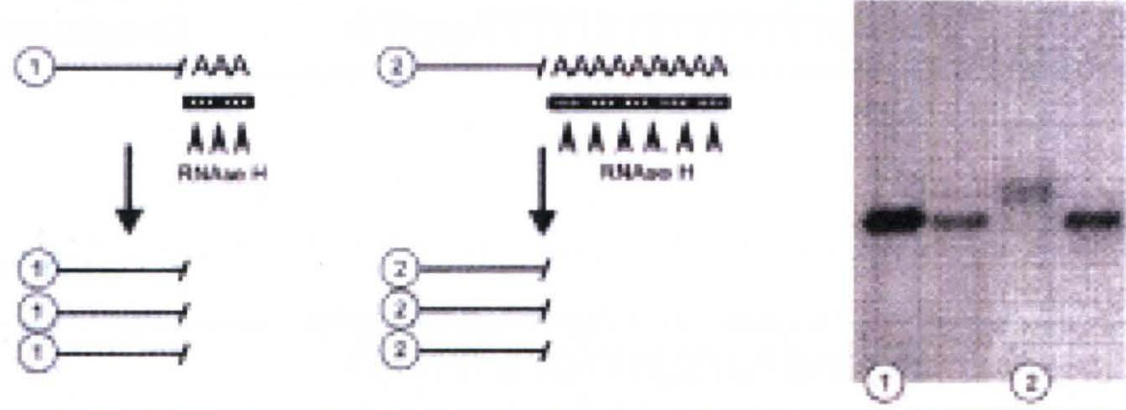

RACE-PAT
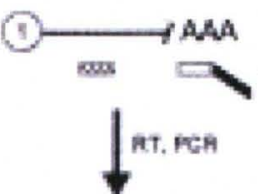

2
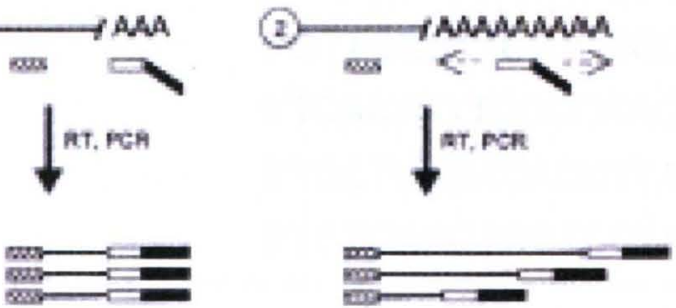

c
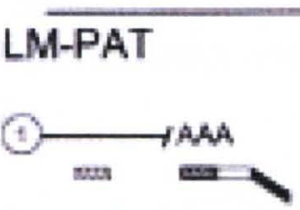

(2)
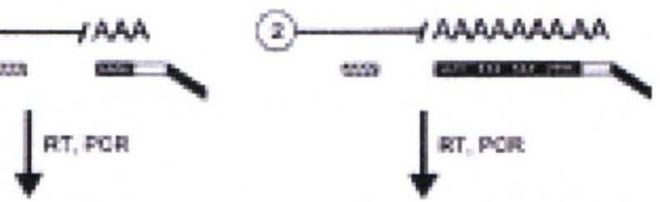

RT PCI
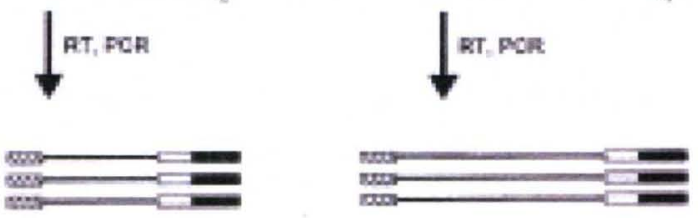

GN $52 \mathrm{hr}$

$-\frac{-10}{0}$

d
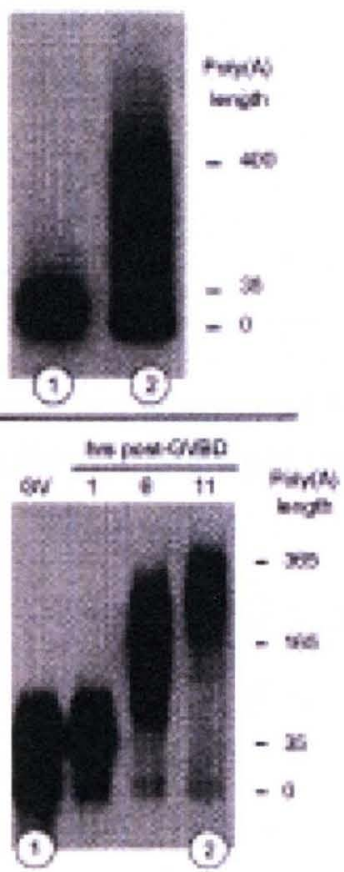
Table 6. Primers used for PAT assay

\section{General primers}

anchored oligo-dT primer

anchor-only primer

5 ' phosphorylated oligo-dT
5'GACTCGAGTCGACATCGATTTTTTTITTTTT'

5'GACTCGAGTCGACATCGA3'

15'Phos/TTTTTTTTTTTTTTTT3'

\section{Gene specific primers}

\begin{tabular}{ll}
\hline BDNF pat & 5'CGTTTCTGTTCGTTCTGGTTC3' \\
CaMKIla pat & 5'GGGAGGGGAGAAGAGATGTC3' \\
MAPK1 pat & 5'TGATGGTGTTATCCCAGTGC3' \\
Ppp2r1a pat & 5'GTTCGGTTCCTCCTCTGTAGC3' \\
Ppp2r2b pat & 5'TCAATGTTCCCCAACTCCC3' \\
IRBP pat fwd & 5'TCCTCAGACACCTTACCTGG3' \\
TTR pat fwd & 5'TCTCCCCTGCTCCTAAAACC3' \\
\hline
\end{tabular}

Note: Anchored oligo-dT primers and 5' phosphorylated oligo-dT primers were used for the reverse transcription step; anchor-only primers and gene-specific primers were used for the PCR step. 
were assembled according to the manufacturer's instructions. PCR was run at the following conditions:

Initial activation: $\quad 95^{\circ} \mathrm{C}, 15 \mathrm{~min}$.

Amplification:

35 cycles of: $94^{\circ} \mathrm{C}, 30 \mathrm{sec}$;

$60^{\circ} \mathrm{C}, 30 \mathrm{sec}$;

$72^{\circ} \mathrm{C}, 30 \mathrm{sec}$;

Final extension: $\quad 72^{\circ} \mathrm{C}, 10 \mathrm{~min}$.

The PCR products were separated via agarose gel electrophoresis. The length of poly $(A)$ tails was indicated by the length of the smear.

8. Quantification of mRNA: real-time PCR

$0.2 \mu \mathrm{g}$ of total retinal RNA from each of the seven developmental ages was used for reverse transcription in preparation of cDNA for subsequent real-time PCR. High capacity cDNA archive kit (Applied Biosystems, Foster City, CA) was used for the reverse transcription to ensure quantitative conversion of RNA to cDNA. Components were assembled according to the manufacturer's protocol. The following temperature and time conditions were used to achieve optimal conversion:

$25^{\circ} \mathrm{C} \quad 10 \mathrm{~min}$

$37^{\circ} \mathrm{C}$ 2hour. 
The resulted cDNA was diluted 1:20 and 5ul of the dilution was used for each real-time PCR reaction that follows. The Gene Expression Assays - the mixture of gene-specific primers and gene-specific, FAM-labeled probes, were optimized to ensure similar amplification efficiencies (Applied Biosystems). 2x PCR master mix containing Taq DNA polymerase, dNTPs and optimized reaction buffer was used (Applied Biosystems). 18S was used as the endogenous control. PCR reactions were performed on ABI 7300 realtime PCR system with the following conditions:

$$
\begin{array}{rr}
50^{\circ} \mathrm{C} & 2 \mathrm{~min} \\
95^{\circ} \mathrm{C} & 10 \mathrm{~min} \\
40 \text { cycles of: } & \\
94^{\circ} \mathrm{C} & 15 \mathrm{sec} \\
60^{\circ} \mathrm{C} & 1 \mathrm{~min}
\end{array}
$$

Data was analyzed using SDS2.1 software (Applied Biosystems). RNA quantity for each sample was first normalized to the quantity of $18 \mathrm{~S}$ in the same sample, and then calibrated to postnatal day 1 (P1) sample to get the relative fold change. The manufacturer's IDs of Gene Expression Assays for each gene are listed in table 7. The locations that the CPEB3 Gene Expression Assay matches on each transcript variant are listed in table 8 . The primers-probe set generates a product spanning two adjacent exons which represents all known CPEB3 transcription isoforms.

9. In situ hybridization

$12 \mu \mathrm{m}$ frozen sections were used for in situ hybridization. For each gene, sense RNA probe (the complementary sequence of the antisense RNA probe) was used as negative 
Table 7. Gene Expression Assays used for real-time PCR

\begin{tabular}{ll}
\hline Gene Expression Assay & Catalog number \\
\hline CPEB1 & Mm00483970_m1 \\
CPEB2 & Mm00616244_m1 \\
CPEB3 & Mm01204296_m1 \\
CPEB4 & Mm00470951_m1 \\
18S & Hs99999901_s1 \\
BDNF & Mm01334047_m1 \\
CaMKIla & Mm00437967_m1 \\
Mapk1 & Mm00442479_m1 \\
Ppp2r1a (PR 65) & Mm00772799_m1 \\
Ppp2r2b (PR 52) & Mm00511698_m1 \\
Rbp3 & Mm00450075_m1 \\
Transthyretin & Mm00443267_m1 \\
\hline
\end{tabular}

Note: the _ $m$ in the catalog numbers indicate that the primers/probe set only amplified mRNA; while the _s indicates that the primer/probe set amplifies within a single exon, so it does not distinguish genomic DNA from mRNA. 
Table 8. Match locations of CPEB3 Gene Expression Assay on each CPEB3 transcript

\begin{tabular}{cccc}
\hline Gene Accession & Exon Boundary & Assay Location & Amplicon Length \\
\hline NM_198300.2 & $4-5$ & 1084 & 79 \\
AK029261.1 & $4-5$ & 1144 & 79 \\
AK044639.1 & $4-5$ & 1264 & 79 \\
AK044639.1 & $4-5$ & 1084 & 79 \\
AB093274.1 & $4-5$ & 1191 & 79 \\
AK147243.1 & $4-5$ & 1552 & 79 \\
AK161513.1 & $4-5$ & 1238 & 79 \\
BC128377.1 & $4-5$ & 676 & 79 \\
\hline
\end{tabular}


control. Primers (IDT, Coralville, IA) used in PCR amplification to generate the template for probes are listed in table 9. PCR products were visualized on agarose gel and the specific band was cut out and purified using QIAquick Gel Extraction Kit (Qiagen, Valencia, CA). The identity of the purified product was confirmed by sequencing analysis, which was later used as template for in vitro transcription to generate DIGlabeled RNA probes (Invitrogen, Carlsbad, CA). RNA probes were denatured at $85^{\circ} \mathrm{C}$ for 5 minutes then chilled on ice. Slides were treated with $0.1 \mathrm{M}$ RNase-free triethanolamine (TEA)- $\mathrm{HCl} \mathrm{pH} 8.0$ for $5 \mathrm{~min}$, washed, and pre-hybridized at room temperature for an hour in hybridization buffer, which was then replaced with hybridization solution containing $100-200 \mathrm{ng} / \mathrm{ml}$ of RNA probes for overnight incubation at $65^{\circ} \mathrm{C}$. On the second day, slides were washed in $0.2 \times S S C$ several times then transferred to buffer $\mathrm{B} 1(0.1 \mathrm{M}$ Tris, $0.5 \mathrm{M} \mathrm{NaCl}, \mathrm{pH} 7.5)$ for 5 minutes. Slides were then incubated in buffer B2 (buffer B1 with 10\% heat-inactivated sheep serum) for 1 hour at room temp, after which buffer B2 was replaced with buffer B2 containing anti-DIG antibody (1:5000) for overnight incubation. On the third day, slides were washed, equilibrated in buffer $\mathrm{B} 3(0.1 \mathrm{M}$ Tris, $0.1 \mathrm{M} \mathrm{NaCl}, 50 \mathrm{mM} \mathrm{MgCl} 2, \mathrm{pH} 9.5)$, and colorization reaction was carried out in buffer B4 (buffer B3 with 20 $\mu / / \mathrm{ml}$ NBT/BCIP stock solution and $0.1 \%$ tween-20). Slides were then mounted in Mowiol mounting medium for observation under the microscope.

10. Isolation of proteins

Frozen retina was homogenized using a sonicator (Biologics, Manassas, VA). Appropriated amount of pre-chilled CelLytic MT Lysis buffer (Sigma, St. Louis, MO) with $1 \%(\mathrm{v} / \mathrm{v})$ protease inhibitor cocktail (Sigma) was added to the frozen tissue. Sample was immediately homogenized on ice at a moderated pulse and moderate power level. 
Table 9. Primers used for in situ hybridization

\begin{tabular}{|c|c|c|}
\hline Probe & Forward primer & Reverse primer \\
\hline $\begin{array}{l}\text { CPEB1. } \\
\text { antlsense }\end{array}$ & $\begin{array}{l}\text { 5'TAGCAATGCCAATATCTTTCGAAGG } \\
3^{\prime}\end{array}$ & $\begin{array}{l}\text { 5'GTAATACGACTCACTATAGGG } \\
\text { TGGTCTGATCCAGAGCTGAA3' }\end{array}$ \\
\hline CPEB1- sense & $\begin{array}{l}\text { 5'GTAATACGACTCACTATAGGGTAG } \\
\text { CAATGCCAATATCTTTCGAAGG3' }\end{array}$ & 5'TGGTCTGATCCAGAGCTGAA3' \\
\hline $\begin{array}{l}\text { CPEB2- } \\
\text { antisense }\end{array}$ & $\begin{array}{l}\text { 5'TGAGCAGCCAGAAGAGGAAAGAGT } \\
\text { T 3' }\end{array}$ & $\begin{array}{l}\text { 5'GTAATACGACTCACTATAGGG } \\
\text { TGTGTTACTATTGTTGTCTG3' }\end{array}$ \\
\hline CPEB2- sense & $\begin{array}{l}\text { 5'GTAATACGACTCACTATAGGGTGA } \\
\text { GCAGCCAGAAGAGGAAAGAGTT 3' }\end{array}$ & 5' TGTGTTACTATTGTTGTCTG3' \\
\hline $\begin{array}{l}\text { CPEB3- } \\
\text { antisense }\end{array}$ & 5'ACAGAGCCAGCTGCGCAAACCA3' & $\begin{array}{l}\text { 5'GTAATACGACTCACTATAGGG } \\
\text { GAAGGTGCCTCCGAAGACCG3' }\end{array}$ \\
\hline CPEB3- sense & $\begin{array}{l}\text { 5'GTAATACGACTCACTATAGGG } \\
\text { ACAGAGCCAGCTGCGCAAACCA3' }\end{array}$ & 5'GAAGGTGCCTCCGAAGACCG3' \\
\hline $\begin{array}{l}\text { CPEB4- } \\
\text { antisense }\end{array}$ & $\begin{array}{l}\text { 5'GGAGGAAAGACGTGAGACATTAGG } \\
\text { T } 3 \text { ' }\end{array}$ & $\begin{array}{l}\text { 5'GTAATACGACTCACTATAGGG } \\
\text { TGTCAACACTGGTGATTCAA3' }\end{array}$ \\
\hline CPEB4- sense & $\begin{array}{l}\text { 5'GTAATACGACTCACTATAGGGGGA } \\
\text { GGAAAGACGTGAGACATTAGGT 3' }\end{array}$ & 5' TGTCAACACTGGTGATTCAA3' \\
\hline
\end{tabular}

Note: The sequences in bold is T7 promoter. 
Samples were then centrifuged at $4^{\circ} \mathrm{C}$ at $1000 \mathrm{xg}$ for 10 minutes to remove tissue debris. Supernatant were collected and transferred to new tubes.

Protein concentration was determined using Bradford assay (Sigma, St. Louis, MO). Briefly, BSA standard (Sigma) of $0.125 \mathrm{mg} / \mathrm{ml}$ to $2.0 \mathrm{mg} / \mathrm{ml}$ was used to generate the standard curve. An $\mathrm{R}^{2}>=0.99$ for the standard curve was considered acceptable. Several dilutions were used for unknown samples. Original concentration of the retina samples were calculated based on the diluted readings that fell within the range of the standard curve. The samples were then dispensed into aliquots and stored in $-80^{\circ} \mathrm{C}$.

\section{Immunofluorescence microscopy}

$12 \mu \mathrm{m}$ frozen sections were used for immunohistochemistry. All steps were carried out at room temperature. Sections were treated with TBS with $0.05 \%$ Triton for fifteen minutes, and then blocked with $10 \%$ normal donkey serum in TBS for 1 hour. Sections were then incubated with the primary antibodies at the indicated concentrations (table 10) for 1 hour. After several washes, fluorescence-conjugated secondary antibodies were applied and incubated for 1 hour. Slides were washed again and subsequently cover-slipped in Vectashield mounting medium (Vector Laboratories, Burlingame, CA) and observed under Fluoview confocal microscope (Olympus, La Jolla, CA). Negative controls with primary antibodies omitted were used for all experiments. Negative controls with primary antibodies pre-adsorbed with an immunogenic peptide or recombinant proteins were applied when possible. 
Table 10. Antibodies used for immunofluorescence

\begin{tabular}{|c|c|c|}
\hline Antibodies & Catalog and Company & Work Concentration \\
\hline CPEB1 & $\begin{array}{l}\text { Custom antibody } \\
\text { Affinity Bioreagents }\end{array}$ & $1: 30,000$ \\
\hline CPEB3 & $\begin{array}{l}\text { Ab10883 } \\
\text { Abcam }\end{array}$ & $1: 1000$ \\
\hline GAPDH & $\begin{array}{l}\text { Img-5019A } \\
\text { Imgenex }\end{array}$ & $1: 5000$ \\
\hline $\begin{array}{l}\text { HRP conjugated secondary } \\
\text { antibody, goat anti rabbit }\end{array}$ & $\begin{array}{l}\text { AP187P } \\
\text { Millipore }\end{array}$ & $1: 30,000$ \\
\hline $\begin{array}{l}\text { HRP conjugated secondary } \\
\text { antibody, goat anti mouse }\end{array}$ & $\begin{array}{l}\text { AP130P } \\
\text { Millipore }\end{array}$ & $1: 30,000$ \\
\hline
\end{tabular}


12. Recombinant proteins

The cDNAs of full-length CPEBs were cloned into pGEX2T vector with a glutathionine Stransferase (GST) tag at the 5' end. The plasmid was transformed to E. coli B21 cells (Keyclone, Cincinnati, $\mathrm{OH}$ ). For the expression of the proteins, B21 cells were cultured in Luria Broth (LB) medium at $37^{\circ} \mathrm{C}$ until the O.D. 600 reached 0.4 . Then isopropyl $\beta-D-$ 1-thiogalactopyranoside (IPTG) was added to the culture to a final concentration of $0.8 \mathrm{mM}$. Cells were allowed to grow for several hours. When the O.D. 600 reached 1.53.0, cells were harvested and proteins extracted using B-PER bacterial protein extraction reagent (Pierce, Rockford, IL). Total proteins (soluble and insoluble proteins altogether) were loaded to polyacrylamide gel electrophoresis (PAGE) gel and stained with Coomassie Blue. The extra band presented in the induced samples compared to the non-induced was purified with the aid of immunoprecipitation. The sequence of the purified band was confirmed by mass spectrometry.

CPEB proteins were predominantly expressed as insoluble forms in inclusion bodies. In order for the recombinant proteins to be used for antibody pre-adsorption, they must be solubilized. Therefore, the following treatments were performed: First, inclusion bodies were purified from the whole cell lysate using B-PER bacterial protein extraction reagent (Pierce); secondly, they were solubilized in Inclusion Body Solubilization Reagent (Pierce); and lastly, solubilized proteins were returned to physiological conditions via dialysis. A brief description of each step was as follows:

For the purification of inclusion bodies, induced culture was collected and centrifuged at 5000xg for 10min. Appropriate amount of Bacterial Protein Extraction Reagent (B-PER) with 1:100 (v/v) proteases inhibitors (Pierce, Rockford, IL) was added to the pellet (cells). Cells were lysed and homogenized by pipetting and vortexing until the suspension was 
homogeneous. The homogenate was centrifuged at $15,000 \mathrm{xg}$ for $15 \mathrm{~min}$ at $4^{\circ} \mathrm{C}$ to separate soluble proteins and insoluble proteins. Appropriate amount of B-PER was added to the pellet (inclusion bodies) and resuspended. Lysozyme was added to a final concentration of $200 \mu \mathrm{g} / \mathrm{ml}$ followed by $5 \mathrm{~min}$ incubation at room temp. 1:10 diluted BPER was added to the tube, vortexed and centrifuged at $15,000 \mathrm{xg}$ for $15 \mathrm{~min}$. Pellets was collected, resuspended in 1:10 B-PER and centrifuged. This step was repeated two more times. The pellet was collected for further treatment.

For solubilization, the pellet of purified inclusion body was resuspended in appropriate amount of Inclusion Body Solubilization Reagent by vigorous vortex or by pipetting. The suspension was shaken for $30 \mathrm{~min}$ and centrifuged at $27,000 \mathrm{xg}$ for $15 \mathrm{~min}$ to remove cell debris. The suspension was the solubilized proteins from the inclusion body. Because the Inclusion Body Solubilization Reagent contains high amount of strong denaturant, proteins in this buffer have to be dialyzed before they can be used for antibody preadsorption.

Dialysis was performed at $4^{\circ} \mathrm{C}$ to maintain protein stability. Solubilized protein samples were put into Slide-A-Lyzer cassettes (Pierce) of appropriate molecular cutoff. The cassette with sample was put in $1 \mathrm{~L}$ of $6 \mathrm{M}$ urea and dialyzed for 6 hours. $250 \mathrm{ml}$ of $25 \mathrm{mM}$ Tris. $\mathrm{HCl}(\mathrm{pH} 7.5)$ was added to the container every 6-12 hours, until the volume reached 3L. The dialysis solution was then replaced with $2 \mathrm{~L}$ of $25 \mathrm{mM}$ Tris $\cdot \mathrm{HCl}(\mathrm{pH} 7.5)$ and $150 \mathrm{mM} \mathrm{NaCl}$, followed by another 6 -hour dialysis. The sample was removed from the cassette and centrifuged to remove any insoluble material. The concentration of the supernatant (the soluble recombinant protein) was determined using Bradford Assay (Pierce). The sample was then ready for use in antibody pre-adsorption. 
13. Immunoprecipitation

$10 \mathrm{mg}$ of total protein from the retina, $10 \mu \mathrm{g}$ of CPEB3 antibody, and $50 \mu \mathrm{l} 50 \%$ protein $\mathrm{A}$ plus slurry (Thermo Fisher Scientific, Rockford, IL) were used for each immunoprecipitation reaction. PBS buffer ( $\mathrm{NaCl} 150 \mathrm{mM}$, phosphate $10 \mathrm{mM}, \mathrm{pH} 7.4)$ was used as wash/binding buffer. The protein sample and the antibody were mixed and incubated on a rotator at $4^{\circ} \mathrm{C}$ for 3 hours. Protein A plus beads (50\% slurry) were washed three times in PBS and added to the antigen-antibody mixture and incubated on a rotator at $4^{\circ} \mathrm{C}$ for overnight. The complex was centrifuged at $2500 \times \mathrm{xg}$ for 3 minutes, washed three times with PBS, and reconstituted in $30-50 \mu l 2 x$ Lamilli sample loading buffer. The complex was then heated at $95^{\circ} \mathrm{C}$ for 5 minutes, and analyzed by gel electrophoresis. Gel was stained with Gel Code Blue (Thermo Fisher Scientific, Rockford, IL) and destained with water, then cut into several portions for mass spectrometry.

14. Quantification of proteins: Western blotting

$25 \mu \mathrm{g}$ of total protein from each sample was used for Western blots. Protein standards of $10 \mathrm{kD}$ to $250 \mathrm{kD}$ (Thermo Fisher Scientific, Rockford, IL) was used as markers. $2 x$ Laemmli buffer (Sigma, St. Louis, MO) was added to the samples and boiled at $95^{\circ} \mathrm{C}$ for 5min. Samples were loaded to SDS-PAGE for gel electrophoresis. Proteins were transferred from the gel to $0.45 \mu \mathrm{m}$ polyvinylidene fluoride (PVDF) membrane using a Semi-dry transfer apparatus (Biorad, Hercules, CA). The blots were blocked with $10 \%$ milk for 1 hour, before primary antibodies were added for overnight incubation at $4^{\circ} \mathrm{C}$. The blots were washed in TBS with $0.3 \%$ tween-20, and then incubated in secondary 
antibody for 1 hour at room temp. The blots were washed thoroughly before enhanced chemiluminescence reagent (Thermo Fisher Scientific, Rockford, IL) was used to detect the signal. The source and dilutions of the primary and secondary antibodies used are listed in table 11. Recombinant proteins or immunogenic peptides were used to preadsorb the antibody at a ratio of 10:1 (mass/mass) to demonstrate the specificity of the antibody. 
Table 11. Antibodies used for Western blots

\begin{tabular}{|c|c|c|}
\hline Antibodies & Catalog and Source & Work Concentration \\
\hline CPEB1 & $\begin{array}{l}\text { Custom antibody } \\
\text { Affinity Bioreagents }\end{array}$ & $1: 400$ \\
\hline CPEB3 & $\begin{array}{l}\text { Ab10883 } \\
\text { Abcam }\end{array}$ & $1: 50$ \\
\hline Map1a & $\begin{array}{l}\text { M4278 } \\
\text { Sigma }\end{array}$ & $1: 200$ \\
\hline ChAT & $\begin{array}{l}\text { AP144-p } \\
\text { Millipore }\end{array}$ & $1: 200$ \\
\hline $\begin{array}{l}\text { Alexa Fluorß } 594 \\
\text { donkey anti-rabbit IgG } \\
(\mathrm{H}+\mathrm{L})\end{array}$ & $\begin{array}{l}\text { A21207 } \\
\text { Invitrogen }\end{array}$ & $1: 200$ \\
\hline $\begin{array}{l}\text { Alexa Fluor(B) } 488 \\
\text { donkey anti-mouse IgG }\end{array}$ & $\begin{array}{l}\text { A21202 } \\
\text { Invitrogen }\end{array}$ & $1: 200$ \\
\hline $\begin{array}{l}\text { Alexa Fluor(B) } 488 \\
\text { donkey anti-goat IgG }\end{array}$ & $\begin{array}{l}\text { A11055 } \\
\text { Invitrogen }\end{array}$ & $1: 200$ \\
\hline
\end{tabular}




\section{CHAPTER IV}

\section{RESULTS}

\section{CPEB1 expression and regulation}

One previous study reported the presence of CPEB1 mRNA in the mouse retina using PCR (Liu et al. 2000). In this study we set out to confirm this initial observation. Additionally, we set out to demonstrate the localization of CPEB1 mRNA and protein in the mouse retina; the expression patterns of CPEB1 during postnatal development of the retina; and potential mRNA targets for $\mathrm{CPEB} 1$ in the retina.

To confirm the previous finding, we first performed regular RT-PCR using a set of primers that were different from the prior study. We used total RNA collected from the retinas of adult mice. The primers we used for CPEB1 had low homology with the other three known CPEBs, and had no significant homology to any other gene. The amplified band was cut out and sequenced for validation. $\beta$-actin primers were used as a positive control and a negative control; the latter had all the components except that the reverse transcriptase was omitted in the RT step. The result demonstrated that CPEB1 mRNA is expressed in retina of the adult mouse (figure 19). The absence of signal in the negative control indicated no contamination in any component of the RT or PCR steps, or during the setup of these reactions. 
Figure 19. Presence of CPEB1 mRNA in the retina

Total RNA from postnatal day 30 mice retina was used. Regular RT-PCR was conducted using CPEB1 primers that span exons to exclude amplification of genomic DNA. $\beta$-actin was used as a positive control. A reaction with only reverse transcriptase omitted for reverse transcription but with all the other components for RT and PCR (with $\beta$-actin primers) was used as a negative control. The amplified band was sequenced for confirmation. The results demonstrated the presence of CPEB1 mRNA in the mouse retina. 
Figure 19

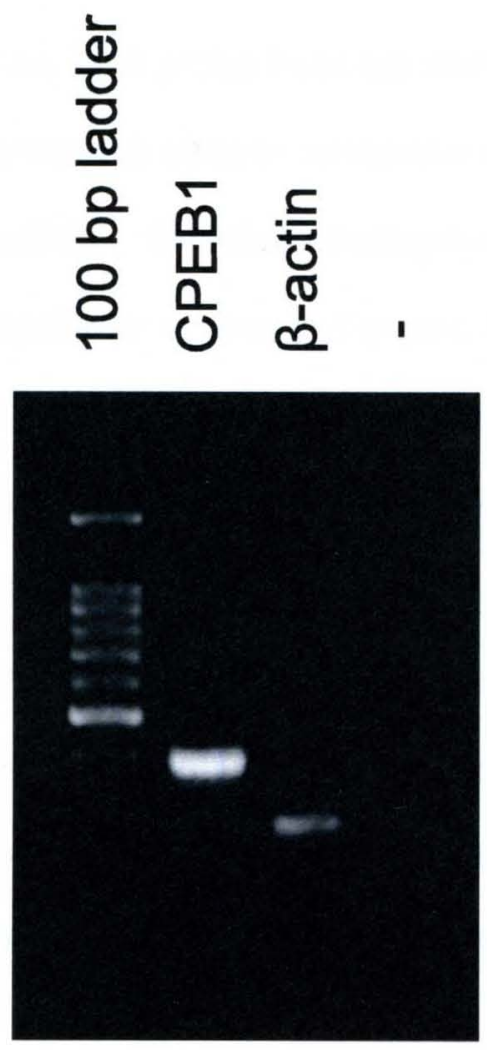


Next, we explored the localization of CPEB1 mRNA in mouse retina with the aid of in situ hybridization. Antisense RNA probes were made with primers specifically designed for CPEB1. The sense RNA probe from the same region was used as a negative control. Amplified templates of both antisense and sense probes were sequenced to confirm the specificity. The clean background in tissue probed with sense probe indicated the specificity of the anti-sense hybridization (figure 20 upper panel). Results in tissue hybridized with antisense probe demonstrated that CPEB1 mRNA is predominantly localized in the inner retina, particularly in the RGC layer, and to some extent, the inner part of the INL (figure 20 lower panel). The IPL and OPL appeared to be positive for CPEB1 as well, but the synaptic structures of these layers could not be distinguished at this point due to the limitation on the resolution of in situ hybridization techniques. The localization of CPEB1 protein would be further explored using immunofluorescence microscopy (see below).

\section{2) Developmental regulation of CPEB1 mRNA}

The next question concerned the possible regulation of CPEB1 mRNA. To explore this issue mRNA levels were compared during postnatal development with the aid of realtime PCR. Total retinal RNAs from seven developmental ages which included the eyeopening event (approximately day 14 in mice) were used. Taking into consideration that physiological responses of the retina to ambient light might cause circadian regulation of certain genes, all the tissues were collected at the same time of the day. Both the 
Figure 20. Localization of CPEB1 mRNA in the retina

P60 mouse retina was used for in situ hybridization. Mouse CPEB1 primers were designed with low homology to the other three CPEBs and to any other mouse genes. The primers were used to make the antisense probe of about $500 \mathrm{bp}$-long whose identify was confirmed via sequencing. The sense probe at exactly the same location was used as a negative control. The results indicated that CPEB1 is highly expressed in RGC layer, and to a less extent, in the INL, especially inner layers of INL. CPEB1 mRNA also appeared to be present in the plexiform layers. Due to the limitation on the resolution of this method, we could not identify synaptic structures in the plexiform layer at this point. The calibration bar represented $50 \mu \mathrm{m}$. 
Figure 20
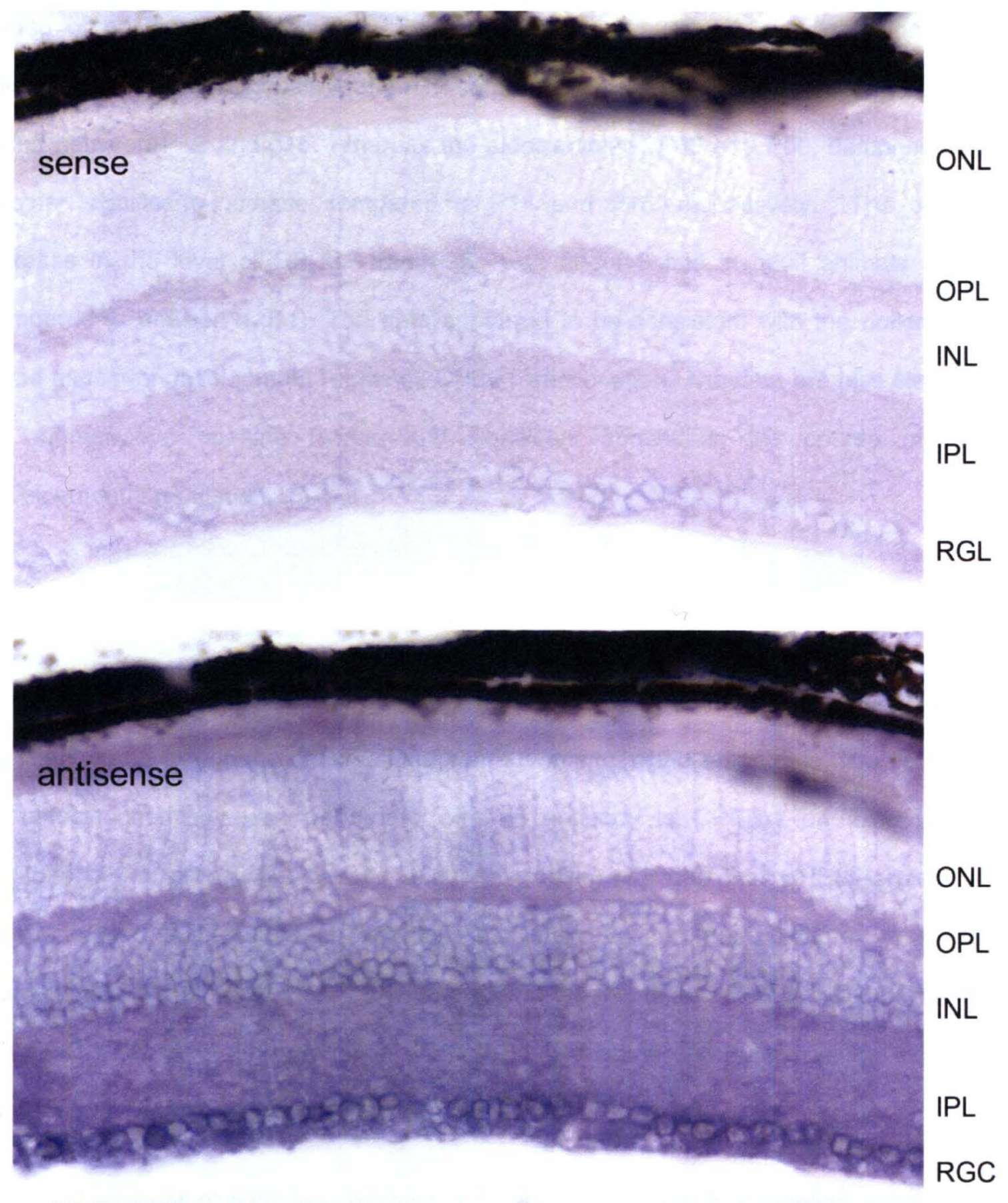
CPEB1 primer set and the 6-carboxyfluorescein (FAM)-labeled CPEB1 probes (Applied biosystems) were gene-specific which ensured stringent specificity.

Our data demonstrated an up-regulation in the level of CPEB1 mRNA throughout the period of postnatal development into adulthood (figure 21). A significant increase $(p<0.05)$ of 2.5 fold was observed in P12 compared to P1. No significant changes were found between P12 and p16. Whereas the older animals, P30 and P60, demonstrated another significant increase compared to $\mathrm{P} 14$ and $\mathrm{P} 16$, respectively. The overall increase in the level of CPEB1 transcripts was about 6 fold in adult animals (P60) compared to newborns (P1). The data appeared to be consistent with the notion that some aspect of development regulates CPEB1 transcription. The data are less clear as to whether eye opening makes any significant impact on the course of this developmental regulation.

3) Localization of CPEB1 protein in the retina

To confirm and extend the observations from the in situ hybridization data, fluorescence immunocytochemistry was performed with an antibody to CPEB-1 on fixed tissue sections of the retina. The results of these studies indicated that CPEB1-protein is clearly located in the neuronal processes that make up the inner plexiform layer. At least two laminar structures are distinguishable in the INL (figure 22, 23). Cell bodies in the RGC layer are immunopositive for CPEB1 and some cell bodies in the INL are immunopositive for CPEB1.

To identify the cell phenotypes expressing CPEB1, we used double labeling, combining CPEB1 antibody with a retinal ganglion cell marker MAP1a (Okabe et al. 1989; Tucker 
Figure 21. Developmental pattern of CPEB1 mRNA

Total RNA from the retinas of postnatal day $1,7,12,14,16,30$, and 60 which were collected at the same time of the day was used for real-time PCR assays. The combination of gene-specific primers and FAM-labeled gene-specific probes ensured high specificity. $18 \mathrm{~S}$ was used as an endogenous control. The relative fold changes were acquired by calibrating all the older ages to age P1. Each age had 6-10 animals. The Anova procedure, Tukey's Studentized Range (HSD) was used for statistics. Error bars indicated standard deviation (SD). Significant changes were found between each two bracketed ages $(p<0.05)$. The results demonstrated an overall up-regulation of CPEB1 transcripts during postnatal development. A significant increase (2.5 fold) was found in P12 compared to P1. P12-16 showed no significant changes. The level of CPEB1 mRNA continued to increase significantly from P16 until the adulthood (P60). The overall increase of CPEB1 transcripts from $\mathrm{P} 1$ to $\mathrm{P} 60$ was about 6 fold. 
Figure 21

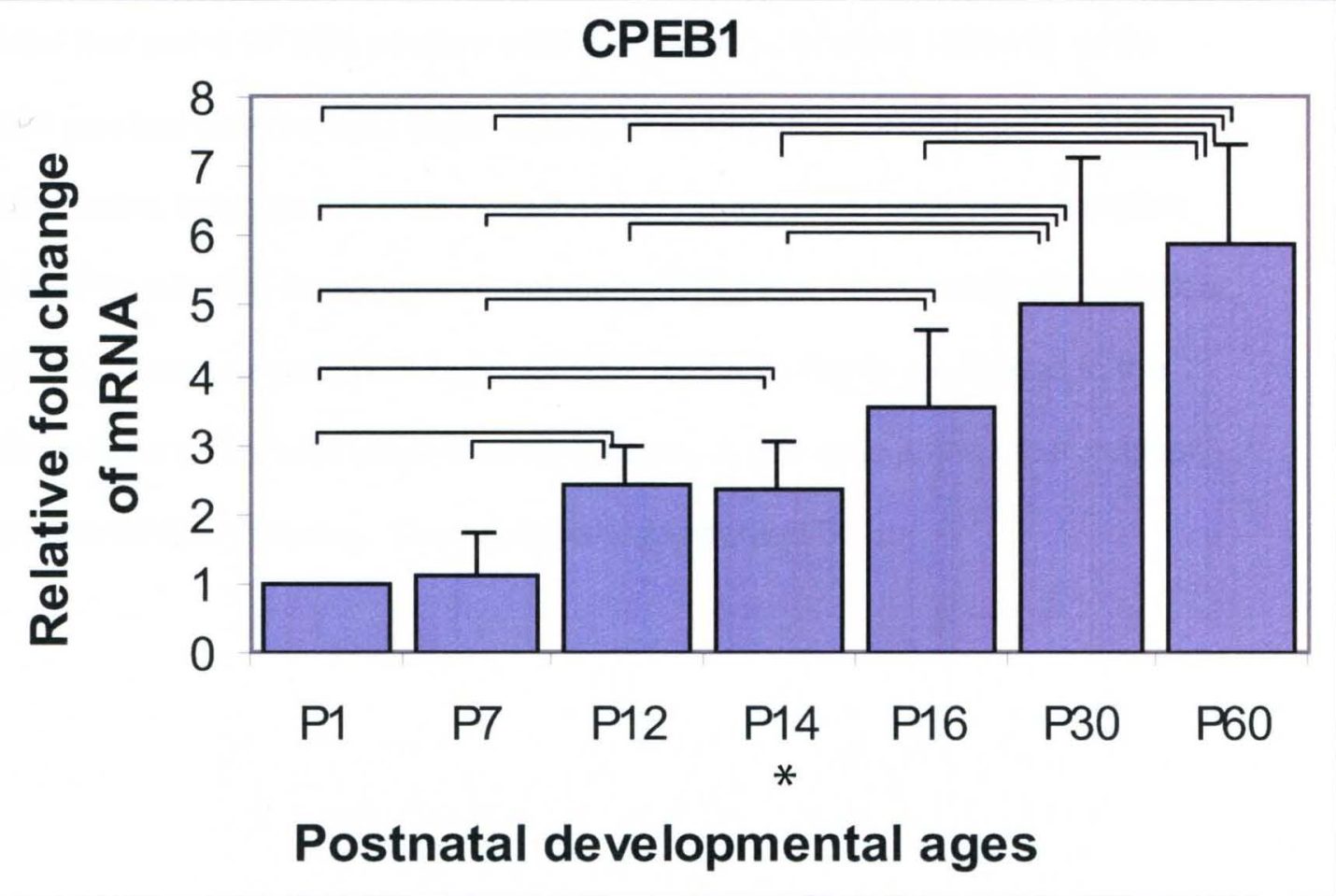


Figure 22. Localization of CPEB1 protein - with MAP1a

P16 retina was used for the immunohistochemistry. CPEB1 was expressed in some cells in the retinal ganglion cell (RGC) layer. The double labeling of CPEB1 and MAP1a demonstrated that some CPEB1 positive cells were MAP1a positive (arrows); while other CPEB1 positive cells in RGC layer were MAP1a negative (arrowheads). This indicated that some, but not all, CPEB1 positive cells in the RGC layer were ganglion cells. The MAP1a labeling in outer plexiform layer (OPL) was non-specific since it also appeared in no primary negative controls. CPEB1 was also highly expressed in the inner plexiform layer (IPL), with some laminar pattern. A few cells in the inner nuclear layer (INL) were CPEB1 positive. The scale bar represented $50 \mu \mathrm{m}$. 
Figure 22

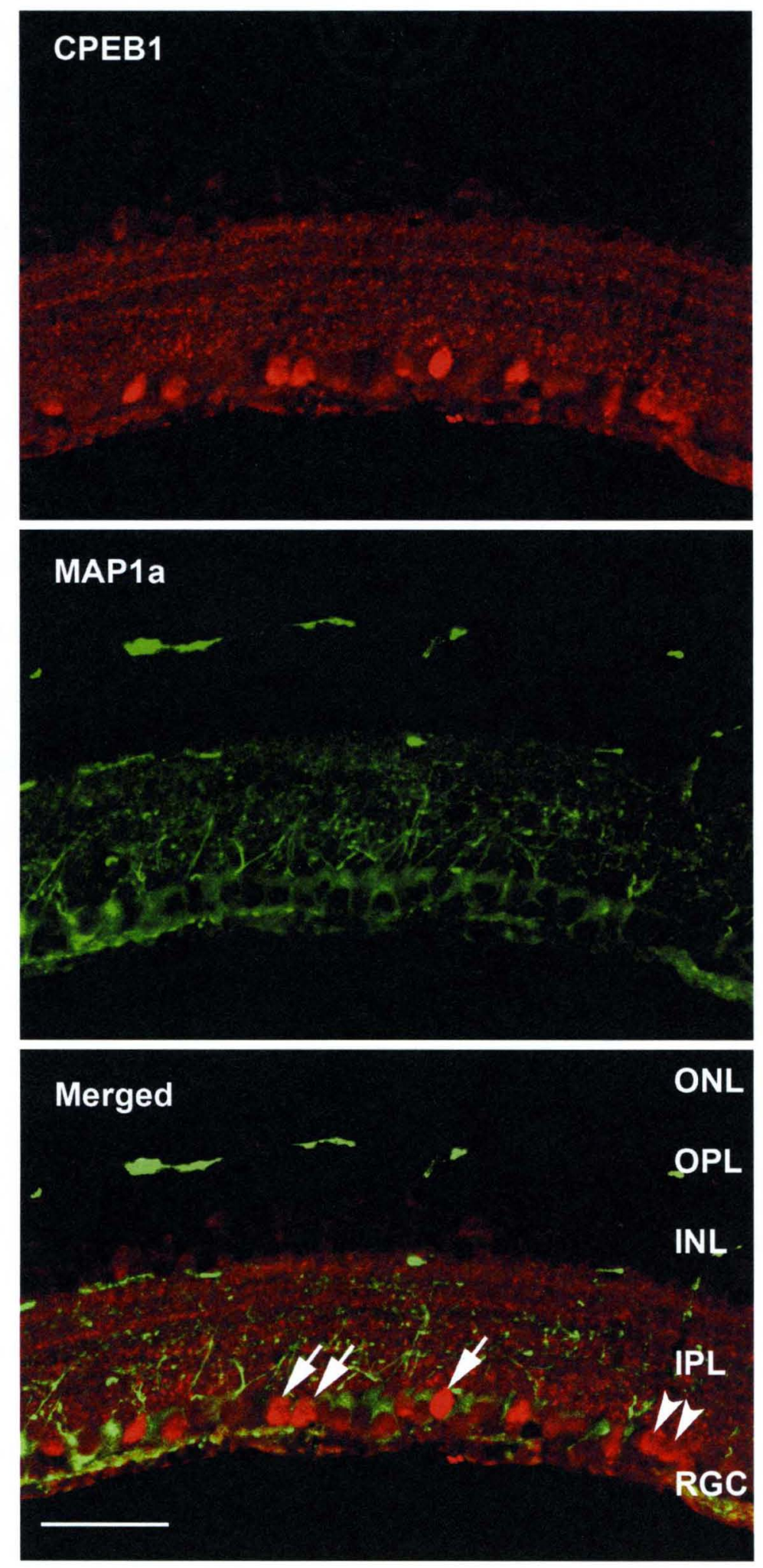


Figure 23. Localization of CPEB1 - with ChAT

P16 mouse retina was used for immunohistochemistry. CPEB1 was expressed in some cells in the retinal ganglion cell (RGC) layer. The double labeling of CPEB1 and ChAT demonstrated that some CPEB1 positive cells were ChAT positive (arrows); while other CPEB1 positive cells in RGC layer were ChAT negative (arrowheads). This indicated that some, but not all, CPEB1 positive cells in the RGC layer were displaced amacrine cells. CPEB1 was also highly expressed in the inner retina, with some laminar pattern. The two distinguishable laminae ( small open arrows) appeared not to be co-localized with ChAT, suggesting that they were not cholinergic synapses. The close juxtaposition of these two laminae with ChAT positive laminae raised the possibility that they may be localized in sublamina $a$ and sublamina $b$ respectively. A few cells in the inner nuclear layer were CPEB1 positive, some being ChAT positive (big open arrows), and others ChAT negative (big open arrowheads). This suggested some of the CPEB1 positive cells in the INL were cholinergic amacrine cell, the others were not. The scale bar represented $50 \mu \mathrm{m}$. 
Figure 23
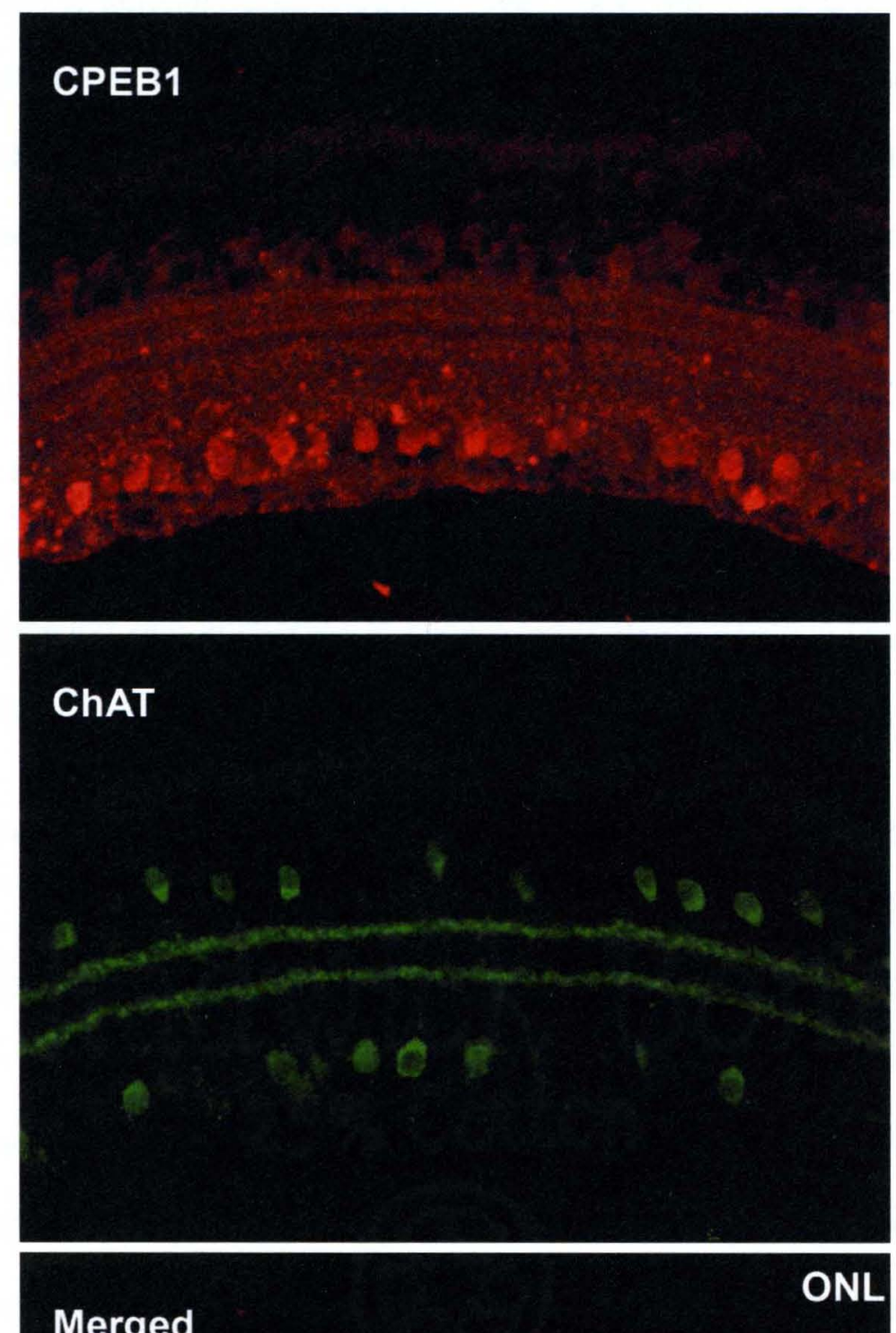

Merged

OPL

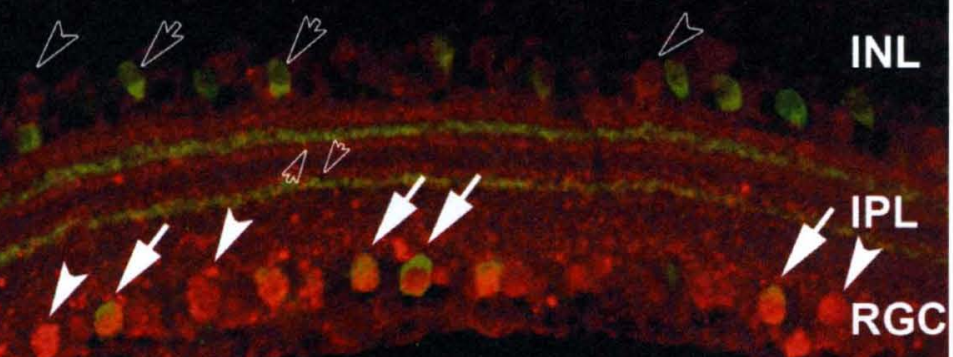


and Matus 1988), or a cholinergic amacrine cell marker choline acetyl transferase (ChAT) (Kang et al. 2004; Eckenstein and Thoenen 1982; Voigt 1986; Zhang et al. 2005). Our data demonstrated that the CPEB1 positive cells in RGC layer can be MAP1a positive (figure 22), or ChAT positive (figure 23). This suggested that some CPEB1 positive cells in the RGC layer are retinal ganglion neurons, and some are displaced amacrine cells. Some, but not all CPEB1 positive cells in the inner layers of INL are ChAT positive, indicating that some of those cells are cholinergic amacrine cells. The CPEB $1+/ \mathrm{ChAT}$ cells in the INL could be other types of amacrine cells, or bipolar cells. To determine all phenotypes of cells expressing CPEBs would require an extensive study of double and triple antibody labeling with antibodies to known cell markers which is beyond the scope of this dissertation.

CPEB1 immunolabeled tissue sections showed distinguishable patterns during development. Throughout all developmental ages examined, CPEB1 was highly expressed in the IPL. The labeling of CPEB1 was across the IPL, but some distinguishable laminar structures were labeled at a higher level. These laminar structures did not appear to co-localize with ChAT labeling (figure 23). This suggested that these synaptic layers are not cholinergic. However, these CPEB1 positive laminae are within close proximity to the ChAT positive laminae. A dark "furrow" lay between two laminar structures in the CPEB1 staining.

4) Developmental regulation of CPEB1 proteins in the retina

To expand our observation of the developing pattern of CPEB1 mRNA, we looked at the levels of CPEB1 proteins during development with the aid of Western blots. To validate the specificity of the antibody, the immunogen, a peptide used to generate the antibody 
was used in pre-adsorption experiments as the negative control (figure 24b). The results from Western blots (figure 24a) indicated that qualitatively the level of CPEB1 protein was up-regulated in the retina during the postnatal development, which is consistent with our observation on the developmental pattern of CPEB1 mRNA (figure 21).

5) Identification of CPE-containing mRNAs in mouse database using a bioinformatics program

Two consensus sequences for CPEs in mRNAs that contain such regulatory sequences have been published. These include: UUUUUAU and UUUUAAU (Fox et al. 1989;

Simon et al. 1992). However, very few gene-transcripts are known targets of CPEB1 in the central nervous system. To identify potential mRNA targets of CPEB1 using the current knowledge of CPE sequences, two approaches were used to explore this relatively new area. The approaches adopted included the use of a home-grown bioinformatics program, and the use of a specialized 3' RACE RT-PCR.

The bioinformatics program was designed with the help and collaboration of Dr. Eric Rouchka in the Department of Computer Engineering and Computer Sciences and named "CPE Detector". This program searched the 3' UTR databases UTRdb ( www.ba.itb.cnr.it/UTR/) for the presence of the CPE consensus motif, the PAS consensus motif, the relative location of the two (CPE may partially overlap with PAS, but is always upstream of PAS), and the length of the gap between the two. Genes with the required characteristics were retrieved and classified into different categories based on the length of the gap (table 12). Several redundant or non-redundant databases ranging from fungus to human were used. From the non-redundant mouse 3'UTR database, we identified a total of 1449 genes that have at least one CPE upstream of the 
Figure 24. Developmental pattern of CPEB1 protein

a) Total retinal proteins of P1-P60 mice were used for western blots. Qualitatively the level of CPEB1 protein in the retina was up-regulated during postnatal development. This is consistent with real time PCR data.

b) To ensure the specificity of the antibody, synthetic immunogenic peptide was used to pre-absorb the antibody before applying the antibody to the blot. Preadsorption diminished the band which suggested that the detected band was specific for CPEB1. 
Figure 24

$\begin{array}{llllllll}\text { P1 } & \text { P7 } & \text { P12 } & \text { P14 } & \text { P16 } & \text { P30 } & \text { P60 } & \text { P60 }\end{array}$

$75 \mathrm{kD}-$

a

b 
PAS. Among them 185 genes have a gap of $10-50 b p, 190$ have a gap of $51-100 \mathrm{bp} ; 127$ have a gap of $101-150 \mathrm{bp} ; 103$ have a gap of 151-200bp. About 1100 genes have a gap longer than $201 \mathrm{bp}$.

With the aid of the mouse UTR database (www.ba.itb.cnr.it/UTR/), we further screened for genes with two or more CPE motifs upstream of the PAS. After refinement of this program, 1045 genes with two CPE motifs and one poly(A) site in their 3' UTRs were identified; only 36 with a spacer of less than 250bp (table 13). Some of the genes thought to be likely candidates were subjected to polyadenylation tests (PAT) (table 14).

6) 3' RACE-PCR to identify CPE containing mRNAs specifically from mouse retina

To identify the potential retinal mRNAs with CPE and PAS in their sequences, a specialized 3' RACE RT-PCR method was developed. Total mouse retinal RNAs were used in two rounds of nested 3' RACE PCR. The first round amplified specifically CPEcontaining mRNAs; while the second round amplified, in the population derived from the first round of PCR, specifically PAS-containing mRNAs. A highly repeatable pattern was acquired with this method (figure 25). Each distinguishable band was cut out, purified, and sequenced. The identities of these sequences were determined by comparing their sequences to the nucleotide database using Blastn (http://blast.ncbi.nlm.nih.gov/Blast.cgi).

One of the possible limitations of this method was that it did not distinguish CPE/PAS within the 3' UTR from CPE/PAS within the other regions of the gene. After sequencing, only one gene from these bands, transthyretin (TTR), truly had a CPE/PAS in its 3' UTR. TTR encodes for a transport protein for retinol. Its mRNA has been localized in the retinal pigment epithelium (RPE) cells. However immunoreactivity for TTR protein has 
Table 12. CPE-containing mRNAs identified using "CPE Detector" program

Number of genes identified from the databases using "CPE Detector" (developed in collaboration with Dr. Eric Rouchka). Redundant (R) or non redundant (NR) 3' UTR databases for different organisms were used. Candidate genes extracted were categorized according to the length of the gap between CPE and PAS. The results derived from mouse and rodent databases were highlighted.

\begin{tabular}{|c|c|c|c|c|c|c|c|c|c|c|c|}
\hline & & \multicolumn{10}{|c|}{ \# of Nucleotides between patterns } \\
\hline & & $\begin{array}{c}\text { Num. } 3^{\prime} \\
\text { UTRs }\end{array}$ & UNFILTERED & 10 to50 & 51 to 100 & 101 to 150 & 151 to 200 & 201 to 300 & 301 to 400 & 401 to500 & $501+$ \\
\hline FUNGAL & NR & 3122 & 54 & 18 & 19 & 12 & 7 & 6 & 2 & 0 & 3 \\
\hline FUNGAL & $\mathrm{R}$ & 673 & 15 & 5 & 5 & 2 & 3 & 1 & 1 & 0 & 1 \\
\hline HUMAN & NR & 35701 & 3335 & 432 & 406 & 332 & 353 & 572 & 490 & 426 & 1842 \\
\hline HUMAN & $\mathrm{R}$ & 31156 & 1913 & 317 & 239 & 191 & 209 & 316 & 271 & 202 & 827 \\
\hline INVERTEBRATES & NR & 23849 & 1178 & 251 & 248 & 187 & 169 & 214 & 165 & 126 & 318 \\
\hline INVERTEBRATES & $\mathrm{R}$ & 5735 & 224 & 60 & 52 & 28 & 38 & 28 & 10 & 22 & 47 \\
\hline MAMMALS & NR & 7789 & 262 & 39 & 35 & 27 & 20 & 36 & 41 & 26 & 112 \\
\hline MAMMALS & $\mathrm{R}$ & 2359 & 42 & 13 & 3 & 6 & 8 & 3 & 12 & 3 & 17 \\
\hline MOUSE & NR & 18342 & 1449 & 185 & 190 & 127 & 103 & 168 & 178 & 152 & 761 \\
\hline MOUSE & $\mathbf{R}$ & 13784 & 517 & 107 & 71 & 59 & 36 & 59 & 50 & 62 & 198 \\
\hline PATENT & NR & 559 & 31 & 7 & 5 & 3 & 1 & 7 & 9 & 2 & 13 \\
\hline PATENT & $\mathrm{R}$ & 257 & 9 & 1 & 2 & 0 & 0 & 1 & 2 & 0 & 4 \\
\hline Plant & NR & 39483 & 395 & 132 & 118 & 83 & 33 & 22 & 16 & 4 & 10 \\
\hline Plant & $\mathrm{R}$ & 19107 & 66 & 32 & 13 & 9 & 3 & 3 & 0 & 1 & 1 \\
\hline RODENTS & NR & 7408 & 305 & 40 & 38 & 39 & 20 & 49 & 45 & 42 & 105 \\
\hline RODENTS & $\mathbf{R}$ & 2709 & 51 & 5 & 6 & 11 & 2 & 4 & 7 & 7 & 18 \\
\hline Viruses & NR & 5667 & 117 & 3 & 23 & 29 & 17 & 17 & 12 & 9 & 80 \\
\hline Viruses & $\mathrm{R}$ & 17086 & 172 & 0 & 16 & 8 & 2 & 13 & 2 & 10 & 142 \\
\hline Vertebrate & NR & & 1721 & 254 & 268 & 260 & 195 & 343 & 250 & 224 & 625 \\
\hline Vertebrate & $\mathrm{R}$ & & 178 & 19 & 36 & 25 & 19 & 38 & 34 & 17 & 42 \\
\hline & & & 12034 & 1920 & 1793 & 1438 & 1238 & 1900 & 1597 & 1335 & 5166 \\
\hline
\end{tabular}


Table 13. List of mouse genes with two or more CPEs upstream of PAS (within 250 bp distance) in the 3' UTR

\begin{tabular}{|c|c|c|c|}
\hline NUMBER & NAME & PREDOMINANTLY EXPRESSED IN & CNS? \\
\hline AB008811 & $\mathrm{N}$-cadherin & & \\
\hline AF031903 & Arl6 & & \\
\hline AF062567 & $\mathrm{Sp} 3$ & & \\
\hline AY089967 & Basigin 2 & & \\
\hline AY484583 & VGFG2573 & & \\
\hline AY484585 & VGFG2573 & & \\
\hline BC006789 & Ets & & \\
\hline \multicolumn{4}{|l|}{ BC022682 } \\
\hline BC024126 & Dimethylglycine dehydrogenase precursor & & \\
\hline $\mathrm{BC} 026127$ & Csnk1e (Casein kinase 1) & Nerve ganglia; prosencephalon; skin & yes \\
\hline BC027797 & Sp3 (Trans-acting transcription factor 3 ) & Mesencephalon; vascular tissue; lymph node & Yes \\
\hline BC029814 & Fos (FBJ osteosarcoma oncogene) & Bone marrow; bone; liver & yes \\
\hline BC030726 & Casc4 (cancer susceptibility candidate 4) & Nerve ganglia; prosencephalon; cerebellum & yes \\
\hline BC031438 & Extl2 (Exotoses (multiple)-like 2) & Basal ganglia; nerve ganglia; skin & yes \\
\hline BC037478 & Extl2 (Exotoses (multiple)-like 2) & Basal ganglia; nerve ganglia; skin & yes \\
\hline BC040201 & Zfp715 (Zinc finger protein 715) & Endocrine gland; lymph node; whole brain & yes \\
\hline BC048845 & Inhbb (Inhibin beta-B) & Female genital; mammary gland; lung & Yes, not top3 \\
\hline
\end{tabular}




\begin{tabular}{|c|c|c|c|}
\hline BC050916 & $\begin{array}{l}\text { Crsp3 (cofactor required for Sp1 transcriptional } \\
\text { activation, subunit 3) }\end{array}$ & Blood; whole brain; ear & yes \\
\hline BC057182 & Brd1 (Bromodomain containing 1) & Bone; whole brain; eye & Yes \\
\hline BC057543 & Lef1 (Lymphoid enhancer binding factor 1) & Thymus; skin; spleen & small \\
\hline \multicolumn{4}{|l|}{ BC060175 } \\
\hline BC064446 & Casc4 (cancer susceptibility candidate 4) & Nerve ganglia; prosencephalon; cerebellum & Yes \\
\hline X69620 & Inhbb (Inhibin beta-B) & Female genital; mammary gland; lung & Yes (not top 3) \\
\hline M60803 & NA & NA & NA \\
\hline K00020 & Ifnb1 (Interferon beta 1, fibroblast) & NA & NA \\
\hline AY435148 & Gent2 (Glucosaminyl (N-acetyl) transferase 2) & Gall bladder; vascular; extraembryonic tissue & Yes (not top 3) \\
\hline AY435149 & Gent2 (Glucosaminyl (N-acetyl) transferase 2) & Gall bladder; vascular; extraembryonic tissue & Yes (not top 3) \\
\hline AY435150 & Gent2 (Glucosaminyl ( $\mathrm{N}$-acetyl) transferase 2) & Gall bladder; vascular; extraembryonic tissue & Yes (not top 3) \\
\hline BC005508 & $\begin{array}{l}\text { Crsp3 (cofactor required for SP1 transcriptional } \\
\text { activation, subunit 3) }\end{array}$ & Blood; whole brain; ear & yes \\
\hline BC018497 & Arl-6 (ADP-ribosylation factor-like 6) & Embryonic tissue; eye; limb & yes \\
\hline ВC040386 & Zfp715 (zinc finger protein 715) & Endocrine gland; lymph node; whole brain & yes \\
\hline BC057313 & Zfp715 (zinc finger protein 715) & Endocrine gland; lymph node; whole brain & yes \\
\hline M31131 & Cdh2 (cadherin 2) & Eye; whole brain; heart & yes \\
\hline
\end{tabular}


Table 14. Sequences and locations of CPE, PAS, and linker of eight CPE-containing mRNAs

\begin{tabular}{|c|c|c|c|c|c|c|c|}
\hline $\begin{array}{l}\text { Gene } \\
\text { name }\end{array}$ & $\begin{array}{l}\text { Accession } \\
\#\end{array}$ & $\begin{array}{l}\text { 1st CPE } \\
\text { sequence }\end{array}$ & $\begin{array}{l}\text { 1st CPE } \\
\text { location }\end{array}$ & $\begin{array}{l}\text { Hex } \\
\text { Sequence }\end{array}$ & $\begin{array}{l}\text { Hex } \\
\text { Location }\end{array}$ & Linker Seq & $\begin{array}{l}\text { Linker } \\
\text { size }\end{array}$ \\
\hline Actin-beta & & $\begin{array}{l}\text { T4 G T15 G2 T4 } \\
\text { G T c T7 A2 T }\end{array}$ & $\begin{array}{l}1488- \\
1536\end{array}$ & TTTAAA & $\begin{array}{l}1690- \\
1695\end{array}$ & $\begin{array}{l}\text { AGTCATTCCAAGTATCCATGAAATAAGTGG } \\
\text { TTACAGGAAGTCCCTCACCCTCCCAAAAGC } \\
\text { CACCCCCACTCCTAAGAGGAGGATGGTCG } \\
\text { CGTCCATGCCCTGAGTCCACCCCGGGGAA } \\
\text { GGTGACAGCATTGCTTCTGTGTAAATTATG } \\
\text { TACTGCAAAAATTTT }\end{array}$ & 154 \\
\hline \multirow[t]{2}{*}{ BDNF } & NM_007540 & T4 A T & $\begin{array}{l}1502- \\
1507\end{array}$ & TATAAA & $\begin{array}{l}1518- \\
1523\end{array}$ & GAACTGCATG & 9 \\
\hline & & T6 A T7 A T5 & $\begin{array}{l}3029- \\
3048\end{array}$ & $\begin{array}{l}\text { AAAAAG } \\
\text { IAAGAAA }\end{array}$ & $\begin{array}{l}3070- \\
3075 \\
13073- \\
3078\end{array}$ & GAGACATTTTGGGAAAAGGAAA(AAA) & $22 / 25$ \\
\hline \multirow[t]{2}{*}{ CaMKIIa } & NM_177407 & T6 A2 T & $\begin{array}{l}4669- \\
4677\end{array}$ & AATAAA & $\begin{array}{l}4780- \\
4785\end{array}$ & $\begin{array}{l}\text { CTGTGGCTGTGAACTTGAATGACCACTGCT } \\
\text { CAAACTTTCTGCTACTGGGGGGGTGGGGG } \\
\text { AGGGGAGAAGAGATGTCTGGTTTATTCTTG } \\
\text { GCGTTTTCAGTGG }\end{array}$ & 102 \\
\hline & & $\begin{array}{l}\text { T6 G T3 G T3 G } \\
\text { T3 G T5 G T3 G } \\
\text { T3 }\end{array}$ & $\begin{array}{l}4879- \\
4909 \\
\text { (2CPE?) }\end{array}$ & AATAAA & $\begin{array}{l}4929- \\
4934\end{array}$ & CAAATCTCCСCTGTTGCAA & 19 \\
\hline MAPK1 & NM_011949 & T4 G T4 C T2G2 & $2763-$ & AATAAA & 2871- & GACATCAATGGAAAATGGGTTCTATAAAGA & 78 \\
\hline
\end{tabular}




\begin{tabular}{|c|c|c|c|c|c|c|c|}
\hline & & T5 A T6 A T4 & 2793 & & 2876 & $\begin{array}{l}\text { CTGCCTGCTAGTATGAACAGCAATGCAATG } \\
\text { CACTTGTAACTC } \\
\text { ATGGA }\end{array}$ & \\
\hline \multirow[t]{2}{*}{ Ppp2r1a } & NM_016891 & T6 G T gtgt & $\begin{array}{l}2180- \\
2187\end{array}$ & & & GTGTCAATTGTGCCA & 52 \\
\hline & & T5 A T4 A T2 & $\begin{array}{l}2203- \\
2215\end{array}$ & AATAAA & $\begin{array}{l}2239- \\
2244\end{array}$ & СCTTTCCСTACTGTACACGGAGA & 24 \\
\hline \multirow[t]{3}{*}{ Ppp2r2b } & NM_028392 & $\begin{array}{l}\text { seven nt off } \\
\text { compare to } \\
\text { NM_027531. }\end{array}$ & & & & & \\
\hline & NM_027531 & $\operatorname{tgtg} \mathrm{T} 4 \mathrm{G} \mathrm{T} 3$ & $\begin{array}{l}1818- \\
1825\end{array}$ & & & $\begin{array}{l}\text { CTAGGTGTCTGCTGCGATAAAATGAGATTG } \\
\text { TCTGTAGTATTTAAGGAGAAAAAGAGATAA } \\
\text { GG }\end{array}$ & 63 \\
\hline & & T10 A2 T & $\begin{array}{l}1888- \\
1900\end{array}$ & AATAAA & $\begin{array}{l}1945- \\
1950\end{array}$ & $\begin{array}{l}\text { TAAGCATTCCATTTGTTTGAAAAAAATCAAC } \\
\text { AACAAAAAAAAAA }\end{array}$ & 45 \\
\hline RBP-3 & NM_015745 & T6 A3 & $\begin{array}{l}4842- \\
4850\end{array}$ & AATAAA & $\begin{array}{l}4920- \\
4925\end{array}$ & $\begin{array}{l}\text { GGAGGACCATGCACCTGTATTGTCTAGCCT } \\
\text { TGACATCTGGAAAGGCTAAGCTGTTGCTGC } \\
\text { TCAGGGCCT }\end{array}$ & 70 \\
\hline \multirow[t]{2}{*}{ Ttr } & NM_013697 & T3 C T6 A T3 & $623-636$ & TTTAAA & $674-679$ & $\begin{array}{l}\text { CСТСАТTTTTСТССССТGСТССТАAАACCC } \\
\text { AАААТTT }\end{array}$ & 38 \\
\hline & & T9 A T & $\begin{array}{l}1006- \\
1017\end{array}$ & AATAAA & $\begin{array}{l}1033- \\
1038\end{array}$ & AACCTTTAGTGACTA & 15 \\
\hline
\end{tabular}


Figure 25. RACE for identification of target mRNAs of CPEB1

Total P60 mouse retinal RNA was used for 3' RACE. An illustration of the 3' RACE method is shown in figure 16. The used of wobbled CPE primers and wobbled PAS primers in the nested 3'RACE reactions generated multiple bands. Each band was cut out, purified, and sequenced to identify its identity. 
Figure 25

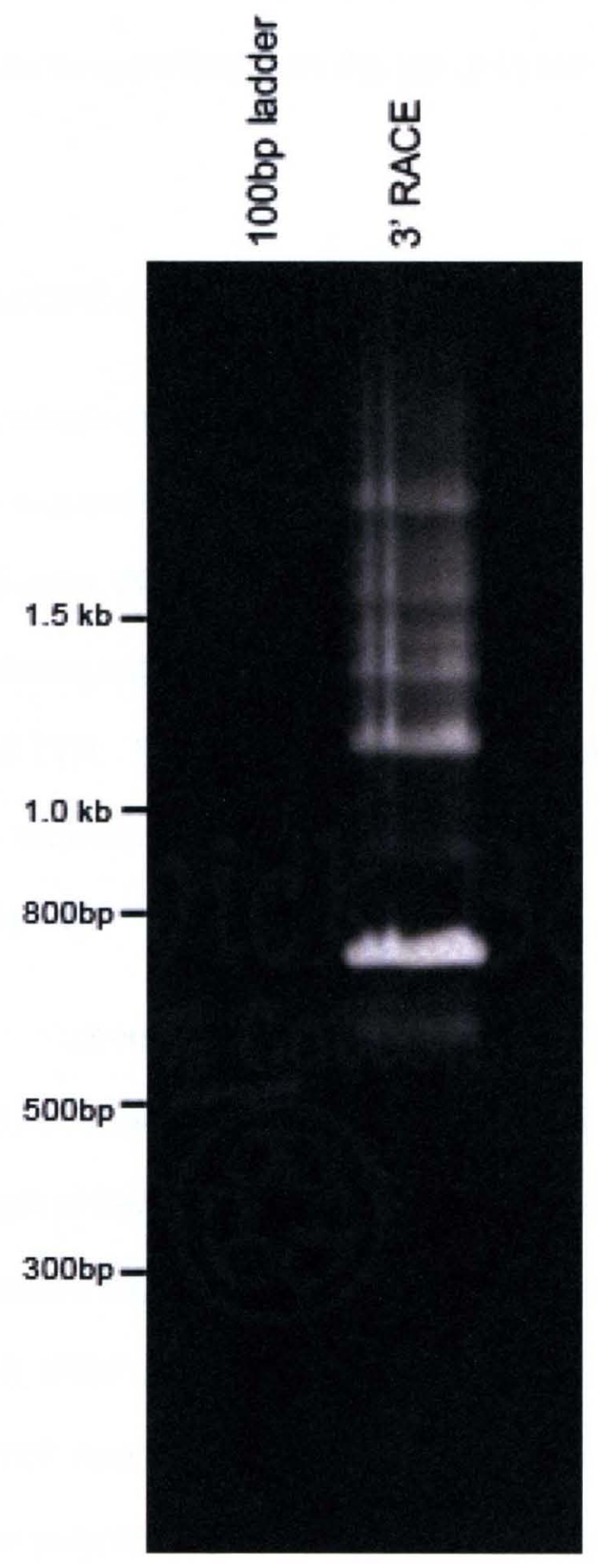


been found in RPE, the optic nerve fiber layer, as well as retinal ganglion cells of the retina (Dwork et al. 1990). This gene, together with several of the genes identified using the bioinformatic program, were used in polyadenylation assays (see below). Another limitation on this method was that because of the competition for the same set of primers, additional genes that have 3' UTR/PAS in their 3' UTRs may not be amplified to sufficient amount for them to be identified from the gel or in our sequence data.

7) Polyadenylation of eight CPE-containing mRNAs in developing retina

The following eight genes, based on their known possession of CPE/PAS motifs in their 3' UTRs and the known or suspected presence in the retina, were selected for the PAT assay: CaMKIla, BDNF, $\beta$-actin, MAPK, protein phosphatase regulatory domain 1a (Ppp2r1a), protein phosphatase regulatory domain 2b (Ppp2r2b), interstitial retinol binding protein (IRBP), and TTR. The number of copies and the sequences of CPE and PAS motifs, as well as the sequence and length of gaps between the two in these eight genes was confirmed (table 14).

The experimental paradigm chosen as a putative trigger for polyadenylation was development. Total retinal RNA samples from mice of different ages were used for the LM-PAT method. The length of the poly $(A)$ tails were visualized on agarose gel (figure 26). The assays were repeated multiple times with consistent results indicating that MAPK1, Ppp2r1A, Ppp2r2B, IRBP and BDNF mRNAs acquired elongated poly(A) tails from $\mathrm{P} 1$ to $\mathrm{P} 7$. Ttr and $\mathrm{BNDF}$ had elongated poly $(\mathrm{A})$ tails from $\mathrm{P} 7$ to $\mathrm{P} 12$. No profound changes in the length of the poly(A) tails were found for these genes in older animals (figure 26). The PAT which measures mRNA elongation provides supporting evidence 
Figure 26. LM-PAT assays of potential CPEB1 targets

Total RNA from seven ages of P1 to P60 retinas were used. An illustration of the LMPAT method is illustrated in figure 17. Eight genes: $\beta$-actin, BDNF, CaMKIla, MAPK, Ppp2r1a (protein phosphatase 2 regulatory subunit A), Ppp2r2b (protein phosphatase 2 regulatory subunit B), IRBP (interstitial retinol binding protein), and TTR (transthyretin) were selected based on the 3' RACE results and the bioinformatics program "CPE Detector". GAPDH, which does not have any CPE sequence in its $3^{\prime}$ UTR, was used as a control. The results demonstrated that from $\mathrm{P} 1$ to $\mathrm{P} 7$, six out of eight candidate genes (except $\beta$-actin and TTR) acquired prolonged poly $(A)$ tail. From P7 to P12, TTR gained prolonged poly(A) tail. 
Figure 26
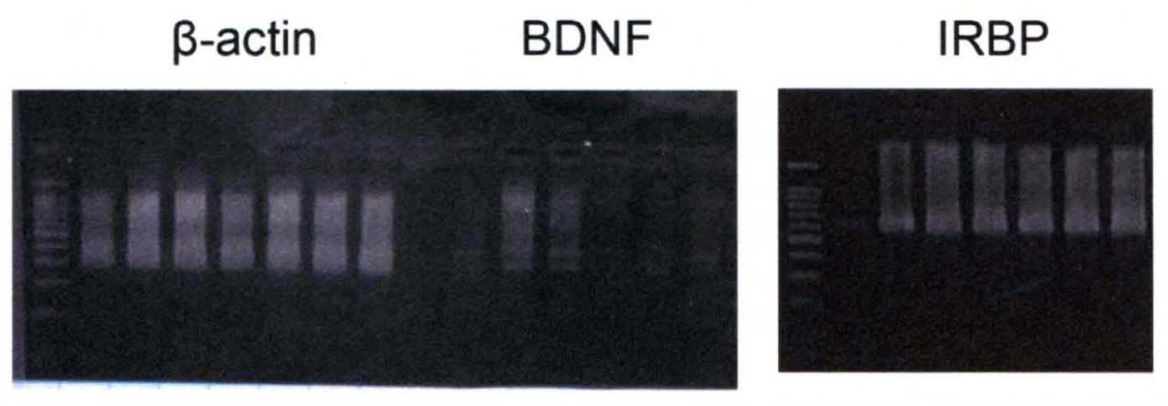

CaMKIIa

GAPDH

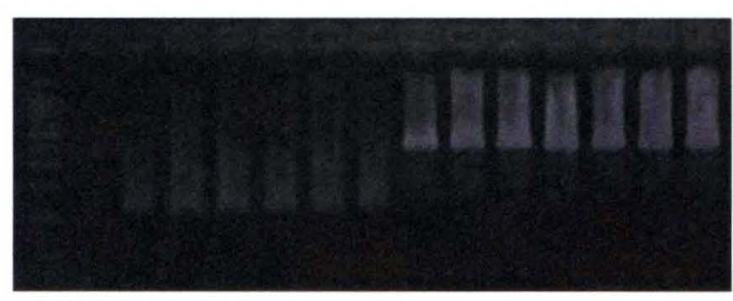

MAPK

Ppp2r1a

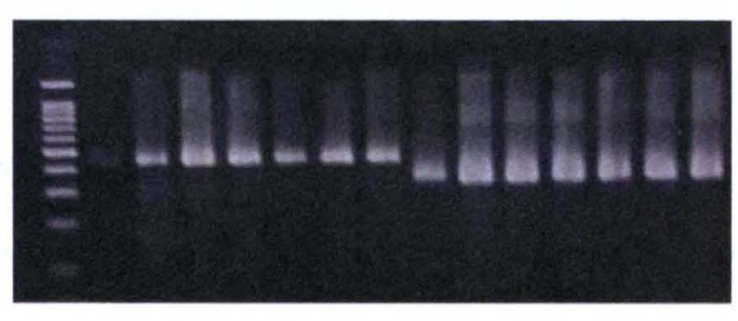

TTR

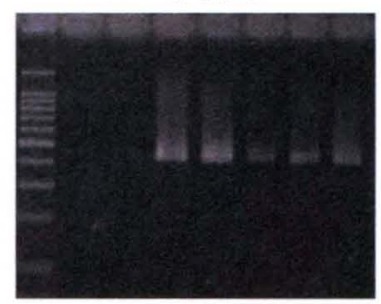

Ppp2r2b

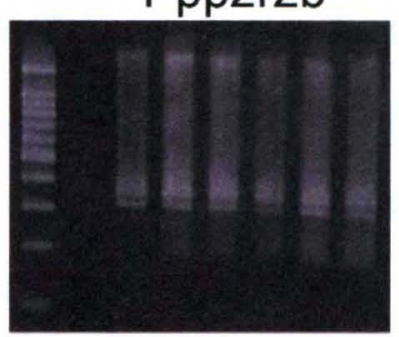


that some aspect of development, especially between P1 and P7 triggers the translational control apparatus.

In summary, our study confirmed the presence of CPEB1 in the mouse retina. The level of CPEB1, both mRNA and protein, were up-regulated throughout development. Potential mRNA targets of CPEB1 were identified both computationally and experimentally. Several genes from the candidate pool acquired elongated poly $(A)$ tails during postnatal development.

\section{CPEB3 expression, regulation and isoform analysis}

Having demonstrated the expression patterns of the well established CPEB1 in the mouse retina, we were particularly interested to see if CPEB3 is present in the retina. One of the recently established target mRNAs for CPEB3, GluR2, has been shown to be a key player in synaptic plasticity. Although much less is known about the mechanism and the expression pattern of CPEB3 compared to CPEB1, it is postulated that CPEB3 functions through a mechanism that is distinct from that of CPEB1. It may also be rather different from CPEB1 with regard to its tissue distribution and its mRNA substrate specificity. The existing state of our knowledge concerning the isoform composition of CPEB3 is summarized in a 2003 publication (Theis et al. 2003). Our goal in this section was to first expand and update this knowledge of CPEB3 isoforms and their relative tissue distribution, and then to establish the expression pattern of CPEB3 in the mouse retina. 
1) CPEB3 has multiple unique motifs compared to CPEB1, 2 and 4.

Four CPEB paralogs (CPEB1-4) derived from four different chromosomes have been identified in mouse. The $\mathrm{N}$ termini of CPEBs are profoundly variable, while the $\mathrm{C}$ termini are highly conserved (Theis et al. 2003). Sequence comparison demonstrates that CPEB3 shares higher homology with CPEB2 and CPEB4, but is distal to CPEB1 in the family tree. RNA recognition motifs (RRM) of CPEB3 share $97-99 \%$ identity with CPEB2 and CPEB4, but only $45 \%$ with CPEB1 (Theis et al. 2003). Searching the complete sequences of CPEB2, 3 and 4 proteins, we identified multiple sequences that are unique to CPEB3, including a poly-glutamine (poly-Q) track, a serine-alanine (SA) track, a 23 amino-acid motif and an 8 amino-acid motif (figure 27). They are also absent from CPEB1. These motifs may have functional significance with respect to the uniqueness to CPEB3.

2) Multiple isoforms of CPEB3 were identified in the database using bioinformatics analysis

A previous study characterized four different alternative splicing isoforms of CPEB3 transcripts (Theis et al. 2003), in which two short motifs of 69nt and 24nt (coding for 23 amino acids and 8 amino acids, respectively) are removed individually, or concurrently. However, the structure of CPEB3 seems to be more variable than previously reported. When investigating the nucleotide databases for CPEB3 isoforms, we were able to identify eight transcript variants (figure 28 , isoform 1a-1d, 3-6). When a similar search was performed for the protein databases, we were able to identify seven distinct CPEB3 protein isoforms (figure 29). With the aid of sequence alignment tools, we mapped the 
Figure 27. Multiple unique motifs of CPEB3 protein

Multiple unique motifs are present in CPEB3 protein, as demonstrated by sequence alignment of CPEB2, CPEB3, and CPEB4 proteins. A glutamine rich motif (poly-Q track), a 23-amino-acid fragment, and an 8-amino-acid fragment (highlighted in blue) are only present in CPEB3. Sequences highlighted in yellow are two RNA recognition motifs that are highly conserved among CPEB2-4. The phosphorylation site of CPEB3 is also indicated in yellow, which seems to be homologous in CPEB4. For simplicity, only RefSeq sequences are used for the alignment. Accession numbers are as following: NP_787951.1 for CPEB2, NP_938042.2 for CPEB3, and NP_080528.2 for CPEB4. Asterisk indicates $100 \%$ conservation; colon indicates substitution with a similar amino acid; period indicates substitution with a distinct amino acid. 
Figure 27

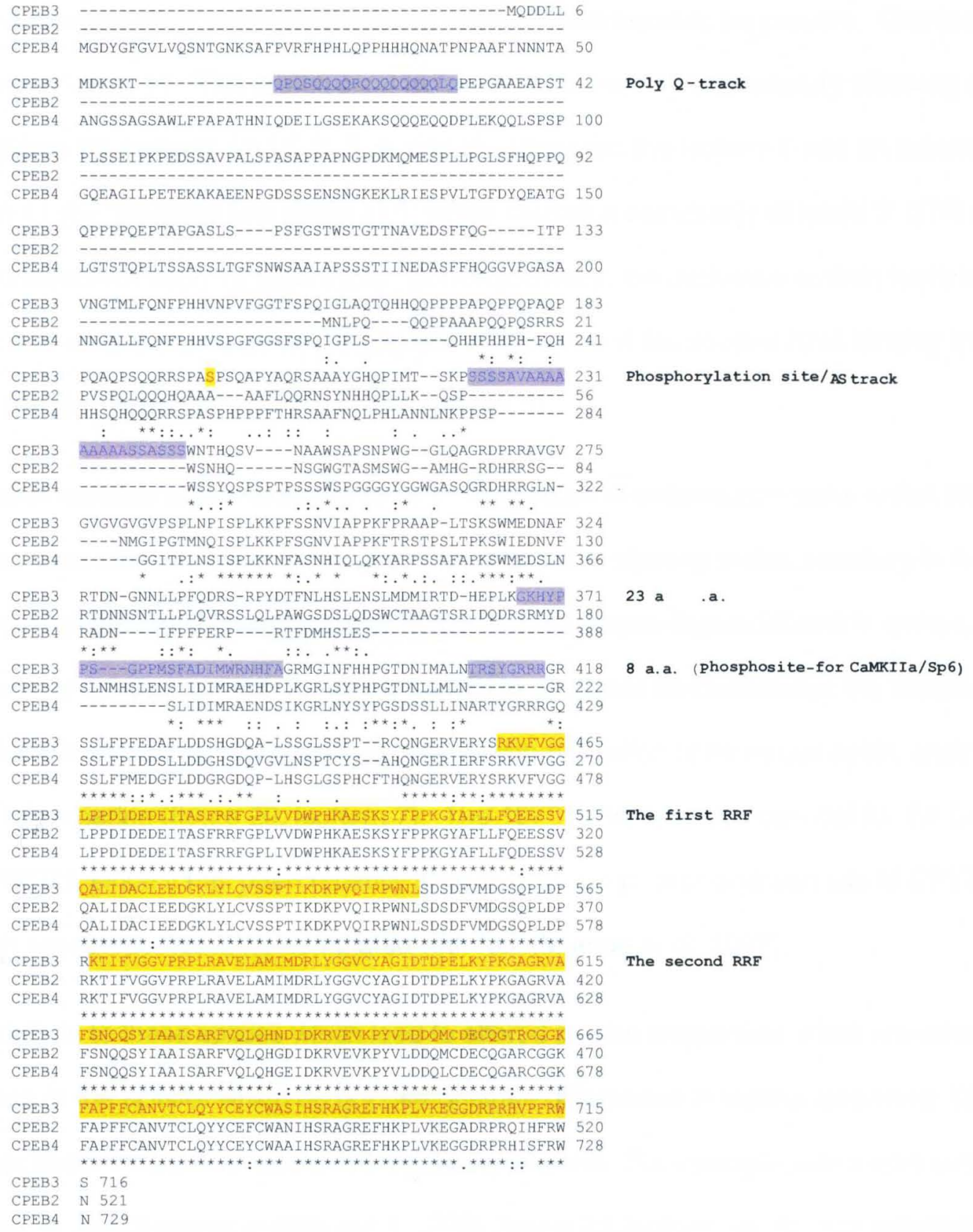


cDNAs to genomic DNA and identified multiple alternatively spliced regions. We also inferred the matches of cDNAs variants to protein isoforms (figure 28,29 ).

Besides the two previously reported sequences whose deletion results in isoforms 2, 3, and 4 , several other alternative splicing regions were found to be present. One location was near exon 11 . The prototypical isoforms have exon 12 immediately following exon 11 (figure 28, isoform 1a-1d, 2, 3, 4, and 6). However, the isoform 5 has an extended exon 11 (by "bleeding into intron 11"), which causes a completely different 3' UTR and the omission of exon 12 (figure 28). Consequentially, the derivative protein terminates with four unique amino acids (VELA) and is deprived of the second RNA binding motif and the zinc finger domain (figure 29, isoform 5).

Another variable region is within exon 4. Two deletion variants can occur within this exon. One deletion leads to the use of an alternative starting codon, resulting in the removal of the first 216 amino acids in the derived protein (figure 28 and 9, isoform 6). The other deletion variant, for which the cDNA could not be identified in the UniGene nucleotide database, has a derived protein with a deletion of 83 amino acids, ending with the 197 th serine (figure 29 , isoform 7). The $197^{\text {th }}$ serine can be essential for the function of CPEB3, since it has been recently shown to be the phosphorylation site of CPEB3 when synaptosomes were stimulated with $\mathrm{KCl}$ (Munton et al. 2007).

In addition to these splices which lead to altered protein sequences, there are other splices in the cDNAs which do not demonstrate differences in protein sequence, but rather show variance in their UTR regulatory regions. For example, alternative usage of exon 1,2 , and 3 results in different 5 ' UTRs (figure 28 , isoform 1a, 1b, 1c); alternative usage of poly(A) signals in exon 13 leads to different 3' UTRs (figure 28 , isoform 1d). 
Figure 28. Analysis of CPEB3 transcript variants in the database

Nine different transcripts of CPEB3 have been reported to the UniGene database or in research paper.

Upper Panel: Representation of genomic sequence with boxes representing exons and lines representing introns. Lower Panel: The cDNAs derived from alternative splicing. Nine cDNAs were shown here, with their accession numbers and types of tissue (when available) given to the right. Partial sequence of isoform 2 (exon 5-7) was confirmed with PCR in a previous study (Theis et al. 2003), but we were unable to identify the accession number in the database, thus its complete sequence shown here was uncertain. The first four isoforms are named as 1a-1d in our study because they code for the same protein. The difference between these four sequences solely reside in untranslated regions. The translational start codons and stop codons were annotated on top of the genomic DNA. The alternative splices of coding exons 4, 5, 7 and 11 generate different proteins presented in figure 29. Alternative splices upstream of the translational start locations (exon 1-3) or downstream of the stop locations (exon 13) give rise to different 5' UTR and 3' UTRs, respectively. 
Figure 28

START1 START2

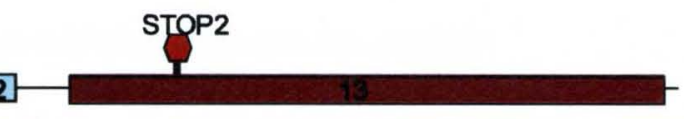

$1 \mathrm{a}$

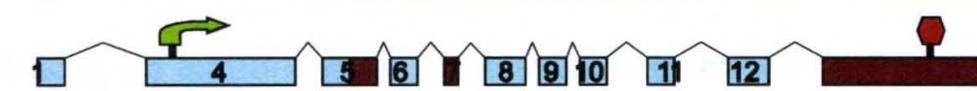

1b

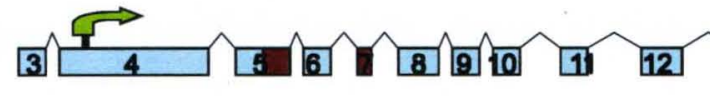

NM_198300

1

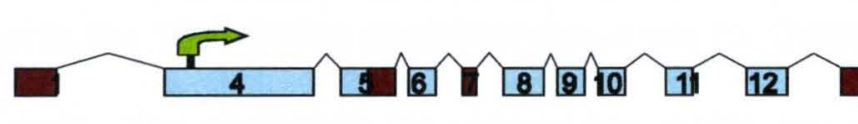

9

$\varphi$

AK147243 adult placenta + brain

1d

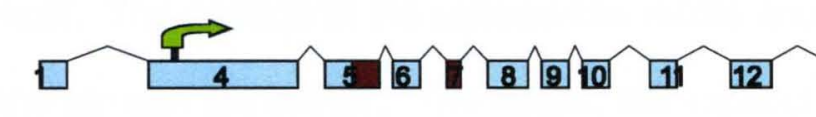

9

AB093274 adult brain

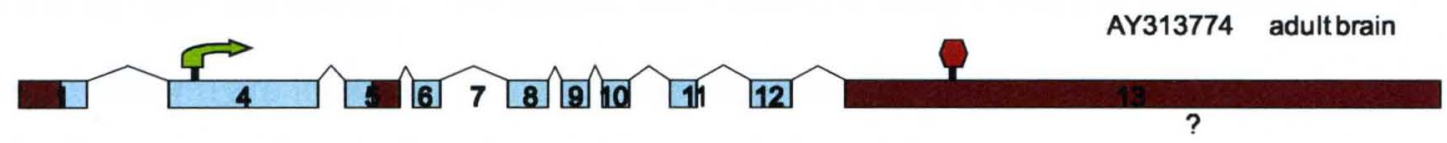

3

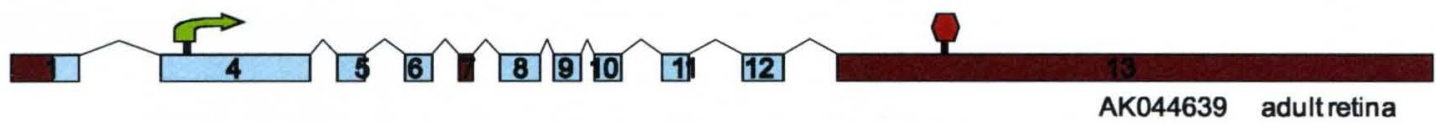

4

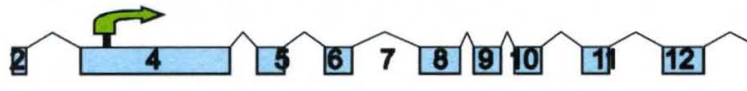

Q

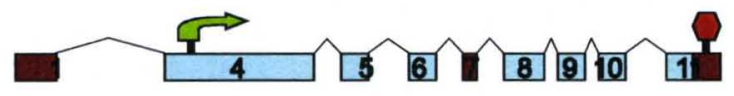

AK029261 dayO neonate head

6

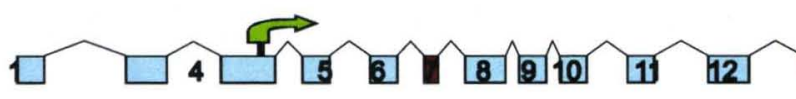

AK161513 adult testis 
Figure 29. Analysis of CPEB3 protein isoforms in the database

Seven isoforms of CPEB3 proteins have been documented in the database.

Isoforms 1-6 corresponded to the same numbered cDNA transcripts in figure 28. Protein isoform 1 was derived from the cDNA variants $1 \mathrm{a}-1 \mathrm{~d}$. The cDNA sequence for protein isoform 7 could not be identified in the database. For simplicity, Poly-Q (poly-glutamine), SA (Ser-Ala track), 23 a.a. and 8 a.a. motifs are only labeled in isoform 1. Colored boxes represent possible functional motifs. Boxes in dash-lines represent deletions. $197^{p}$ represents the $197^{\text {th }}$ serine phosphorylation site for CPEB3. RRM represents RNArecognition motif. The position of the colored-box motifs and dash-line-box motifs are indicated at the top and the bottom. The length, the molecular weight and the accession number of each isoform are indicated to the right (Swiss-Prot/TrEMBL number is unbracketed, NCBI number bracketed). 
Figure 29

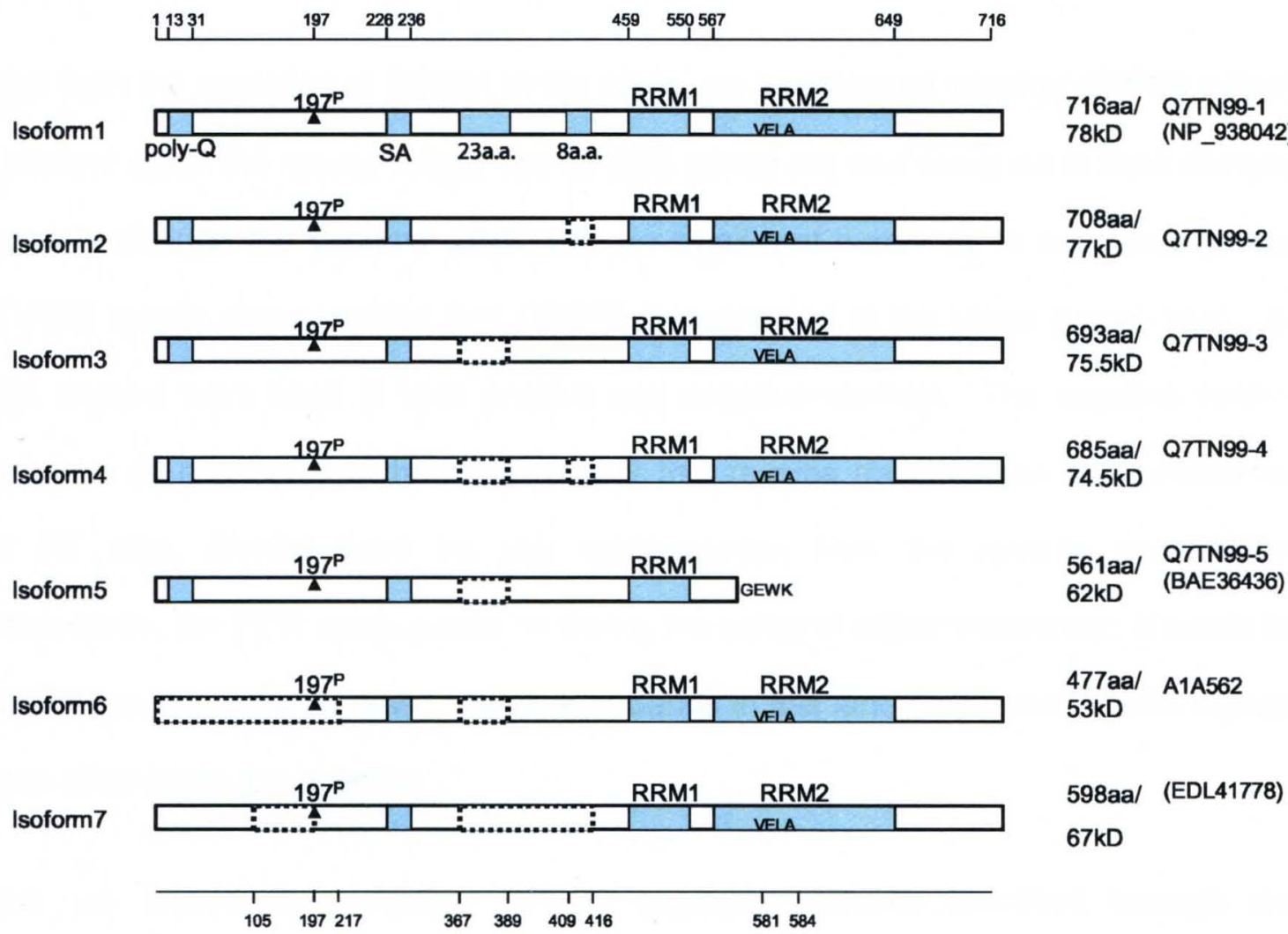


3) Multiple isoforms of CPEB3, including a novel isoform, were experimentally identified in the mouse retina. These isoforms have a wide tissue distribution; and only the $(+69+24)$ variant is selectively expressed among different tissues.

Apart from the presence of CPEB1 in the retina, we investigated whether CPEB3 mRNA is present within the mouse retina. The CPEB3 primer set was designed to flank multiple exons to exclude the genomic DNA, with no significant homology to any other genes. RT-PCR results demonstrated that CPEB3 is expressed in the retina (figure 30a). $\beta$ actin primers were used in both positive and negative controls. The negative control contained all necessary components except that reverse transcriptase was omitted for the RT step. Should there be any contamination from the reverse transcription components, the PCR components, or during the setup of either experiment, it would be amplified in the negative lane. The lack of bands in this lane confirmed that the signals in the other lanes are specific.

Next, we investigated whether CPEB3 transcript variants identified through the bioinformatics analyses were present in the mouse retina. Total RNA isolated from P60 mice retinas and primer sets designed spanning exon 5-7 or exon 11 (figure $30 \mathrm{~d}$ ) were used for RT-PCR. Amplicons of the PCR reactions were separated on agarose gel, and individual bands were extracted and sequenced to confirm their identity. Since partial sequences instead of complete sequences were amplified here, one amplicon may correspond to more than one of the transcripts (figure 28). Therefore, in this section, we used descriptional names instead of numerical isoform names for each amplicon. " $+69+24$ " (corresponding to figure 28, isoform 1a-1d), "-69+24" (figure 28 , isoform 3, 5 , and 6), "+69-24" (figure 28, isoform 2), and "-69-24" (figure 28, isoform 4) were used to designate each variant in the exon 5-7 region; and "extended exon 11" (figure 28, 
Figure 30. Tissue distribution of CPEB3 transcript variants

a) CPEB3 is present in the P60 retina. Both the primers in CPEB3 and $\beta$-actin span multiple exons to discriminate genomic DNA.

b) RNA from adult retina was reverse transcribed and different primer sets for various CPEB3 isoforms (sequences listed in table1) were used for PCR. Each visible band was purified and sequenced to confirm its identity. This data demonstrated the presence of five variants: "+69nt+24nt" (lane2, upper band), "69nt+24nt" (lane2, lower band), "exon11+intron11 bleeding" (lane3), "exon11+exon12" (lane 4, upper band); and a new isoform: "-exon11" (lane 4, lower band). But it does not rule out the presence of "+69-24" and "-69-24", since the competition for the same set of primers by a more dominant variant ("-69+24") may mask the weakly expressed variants.

c) Demonstration of "+69-24" and "-69-24" transcripts and comparison of tissue distribution of all variants. Unique primer sets for each individual variant were used for PCR on thirteen different adult mouse tissues. Each specific band was cut out and sequenced for confirmation. The results demonstrated the presence of "+69-24" and "-69-24" variants in the retina (in supplement to figure b). It also demonstrated the ubiquity of the majority of the variants, with the exception of " $+69+24$ " which was expressed in the central nervous system, the ovary, testis, kidney and heart but absent in the lung, liver, thymus and spleen. Negative controls indicated no contamination from either the RT or the PCR reactions.

d) Mapping of the location of primers used in b) and c) to the CPEB3 cDNA. The sequences of these primers were listed in table 4. CPEB3 isoforms corresponding to each variant were listed to the right. 
Figure 30
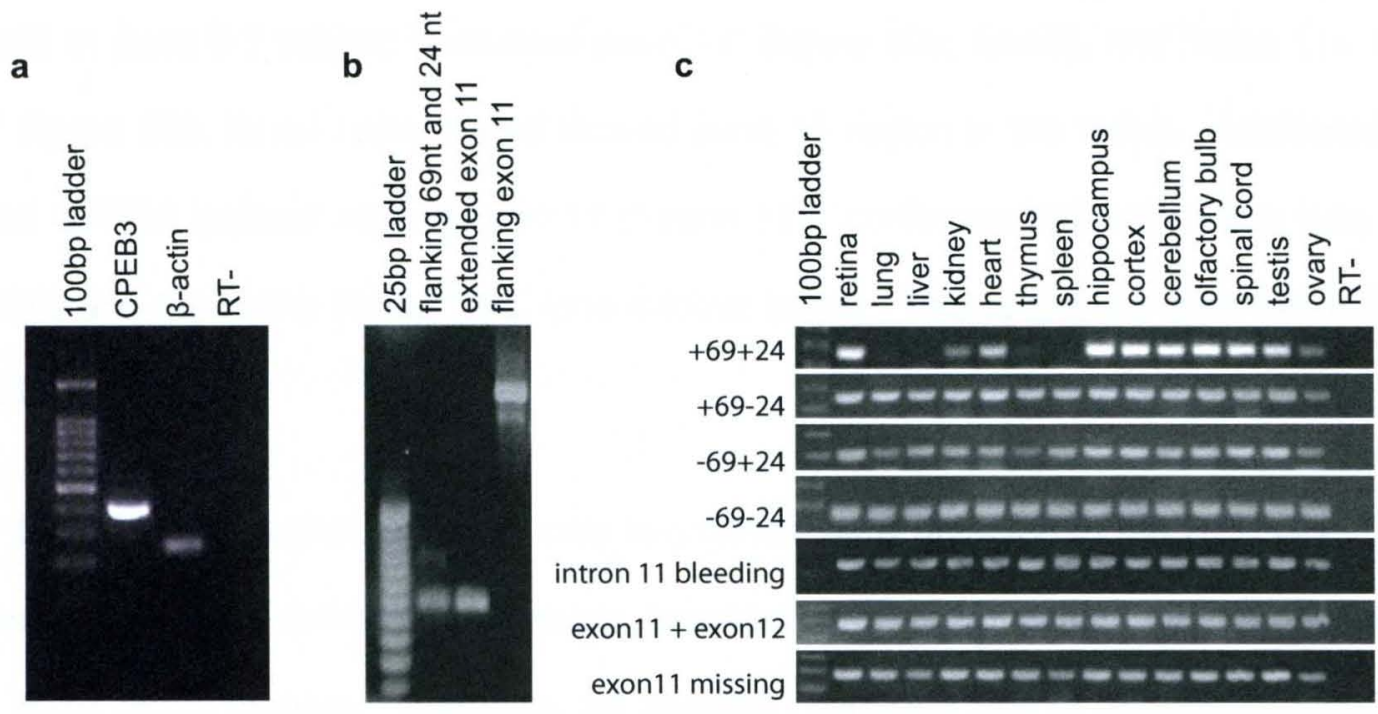

d

(Figure b) Amplicon

flanking $69 \mathrm{nt}$ and $24 \mathrm{nt}$

Primer Set

Intron 11 bleeding

4-5

3-8

flanking exon 11

1-7

(Figure c) Amplicon

$+69+24$

$+69-24$

Primer Set

$17-18$

$17-20$

$-69+24$

$-69-24$

19-18

$19-20$

intron 11 bleeding

exon $11+$ exon 12

3-8

21-13

exon 11 missing

16-1

Corresponding Isoforms (figure 28) isoform 1a-1d

isoform 2

isoform 3, 5, 6

isoform 4

isoform 5

isoform 1a-1d, 2, 3, 4, 6

a novel isoform, not present in figure 28

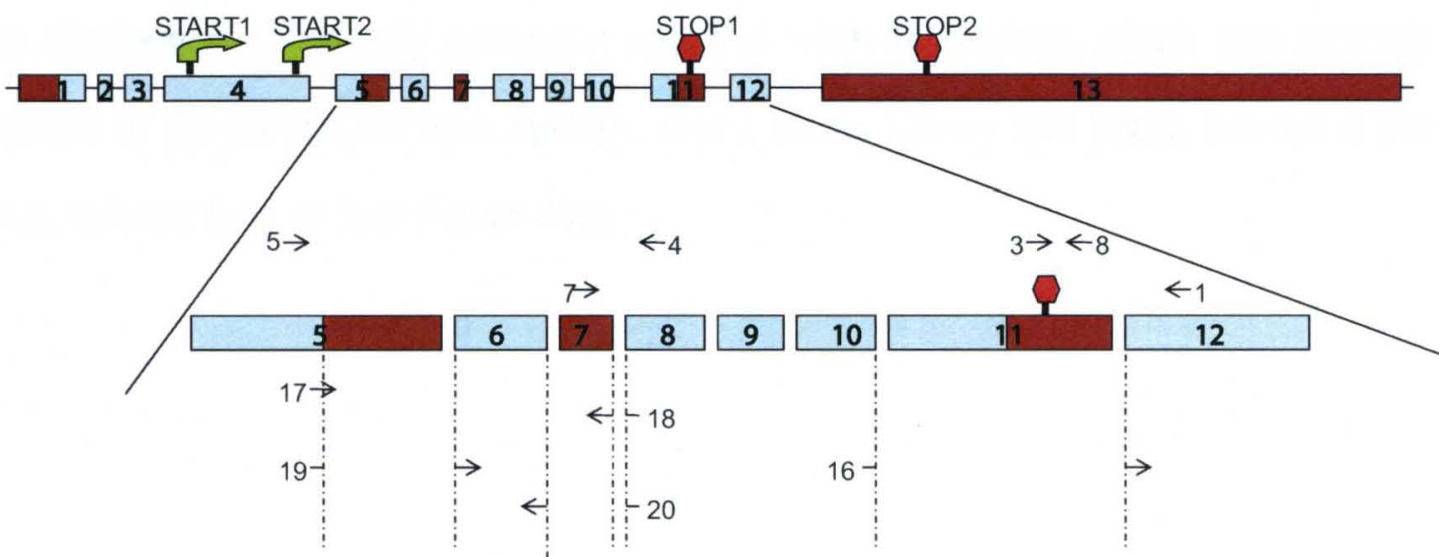


isoform 5), or "exon 11+exon 12" (figure 28, isoform 1a-1d, 2, 3, 4, and 6) for variants near the exon 11 region.

Our results from RT-PCR demonstrated the presence of "+69+24", "-69+24" (figure $30 \mathrm{~b}$, lane2) in exon 5-7 region; "extended exon 11" (figure 30b, lane3), and "exon 11+ exon 12" (figure 30b, lane4 upper band) around exon 11 region in the retina. Additionally, a novel CPEB3 isoform without exon 11 (“-exon 11"), confirmed by sequencing, was also identified in the retina (figure 30b, lane 4 lower band). This is the first time this isoform has been shown.

For the exon 5-7 region, although only two bands were detected in the RT-PCR, it was possible that more dominant transcripts "masked" weaker transcripts by competition for the same set of primers. Therefore, we designed separate sets of primers for individual transcripts in this region to further explore their presence or absence (figure 30d). Each specific band was cut out and sequenced for confirmation (data not shown). Our results demonstrated that the "+69-24" and "-69-24" variants were indeed present in the retina (figure 30c).

We also collected multiple tissues to test all known transcripts for possible tissue specificity. Of all the variants tested, the majority did not appear to differ with respect to tissue distribution. The only exception was the " $+69+24$ " variant, which was strongly expressed in the central nervous system, ovary, testis, kidney and heart, but not in the thymus, spleen, lung or liver (figure 30c). 
4) Localization of CPEB3 mRNA within the retina: the CPEB3 transcripts are expressed predominantly in the retinal ganglion cell layer

With the aid of In situ hybridization, we identified which types of cells in the retina express CPEB3 mRNA in the P60 mouse. To ensure specificity, probes were designed in a region where little homology was found between CPEB3 and the other CPEBs. A sense probe at the exact location was used as a negative control. The primers used to generate the sense and antisense probes were listed in table 3 . Our results demonstrated that CPEB3 is localized predominantly in the RGC layer, and to a less extent, in the inner margin of the INL (figure 31). CPEB3 appeared to be present in the plexiform layers as well, but no synaptic structure was identifiable due to the limitation on the resolution of in situ hybridization technique.

5) CPEB3 transcription is up-regulated during postnatal development of the retina

To investigate the expression of CPEB3 during development, real-time PCR was used to evaluate the relative amount of CPEB3 mRNA in the retinas of mice at different ages. An amplicon of 79 nt spanning exon 4 and exon 5 was amplified, and was inclusive of all CPEB3 isoforms (table 8). Our results (figure 32) demonstrated that the overall transcription level of CPEB3 was up-regulated thoughout postnatal development in the retina. A significant increase in the level of CPEB3 transcripts was seen around the eye opening time (P14). The level of CPEB3 transcripts continued to increase after eye opening, and until adulthood (P60). Since the CPEB3 primer set for real-time PCR matched all known CPEB3 isoforms, the data did not distinguish each transcript variant. 
Figure 31. Localization of CPEB3 mRNA in the retina

14um thick cryostat sections of P60 mouse retina were used for in situ hybridization of CPEB3. The probes were designed in a region with no significant homology to the other three CPEBs to ensure probe specificity. The expression of CPEB3 mRNA was predominantly located in the RGC layer and to a lesser extent, the INL. The IPL appeared to be labeled as well, but the synaptic structures could not be recognized due to the limitation on the resolution of in situ hybridization techniques. Scale bar represented $50 \mu \mathrm{m}$. ONL: outer nuclear layer; OPL: outer plexiform layer; INL inner nuclear layer; IPL: inner plexiform layer; RGC: retinal ganglion cell layer. 
Figure 31

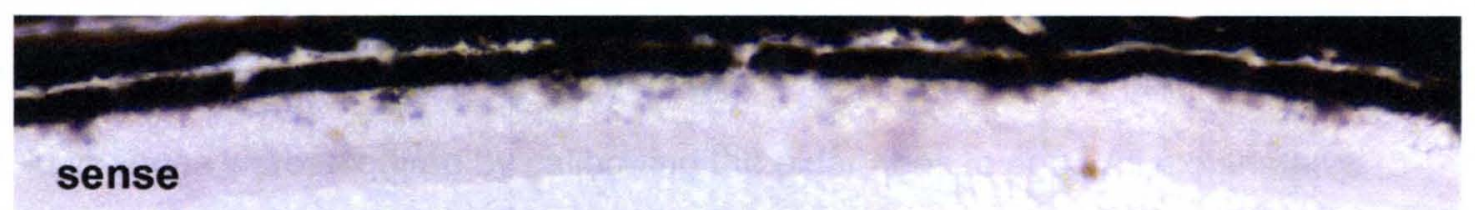

ONL

OPL

INL

IPL

RGC

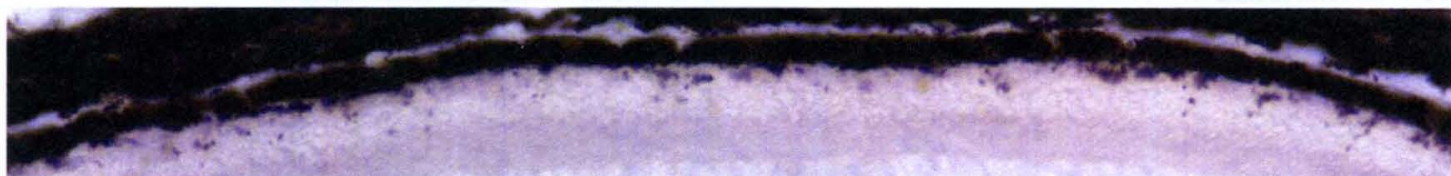

antisense

ONL

OPL

INL

IPL

RGC 
Figure 32. Developmental pattern of CPEB3 mRNAs

CPEB3 mRNA was up-regulated during postnatal development. Seven post natal ages flanking the eye-opening event were used for this study. For each sample the level of CPEB3 mRNA was normalized to that of $18 \mathrm{~S}$ mRNA in the exact same sample. The relative fold changes were acquired by calibrating the older ages to age. All experiments were repeated three times. Statistically significant differences were indicated between two bracketed ages $(p<=0.05)$. The Anova procedure Tukey's Studentized Range (HSD) was used. For each age, the number of samples $n>=6$. Error bars represented the standard deviation. The asterisk indicated the approximate time of eye-opening. The results demonstrated that $\mathrm{CPEB} 3$ was significantly up-regulated in the retina during postnatal development and reached its maximum in adult mice (day 60). 
Figure 32

CPEB3 transcription is upregulated in the developing retina

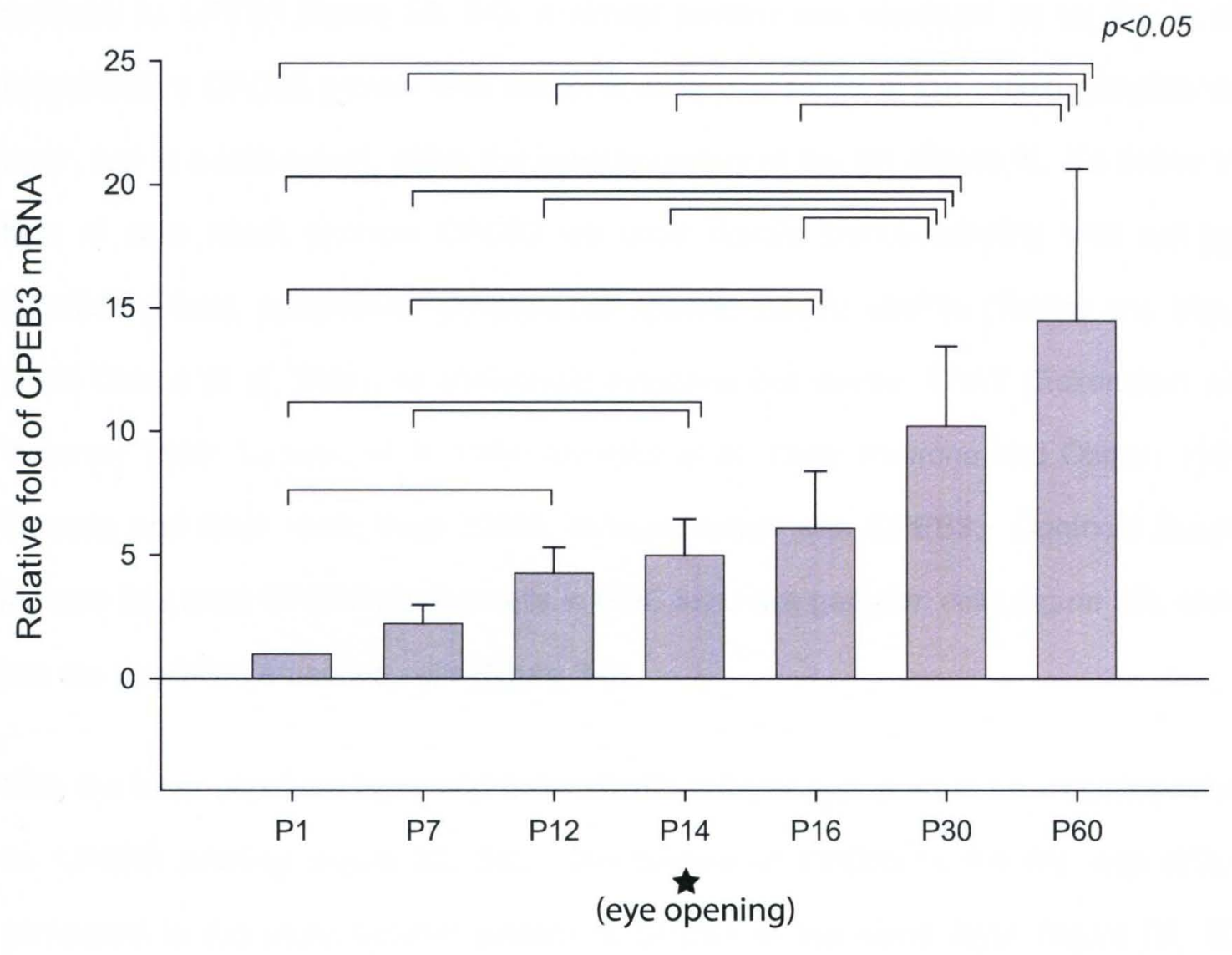

Postnatal Developmental Ages 
6) Localization of CPEB3 protein: CPEB3 protein is predominantly expressed in the inner retina

To confirm the expression pattern of CPEB3 mRNA and to better define the cell types containing CPEB3 protein, we performed fluorescence immunocytochemistry using an antibody to CPEB3 (figure 33,34 ). A similar pattern was observed as for the in situ hybridization: CPEB3 protein was predominantly expressed in the retinal ganglion cell layer, and to a less extent, within the inner boundary of the INL (figure 8). To define the type of cells which express CPEB3 we used double immunolabeling with cell-type specific markers, specifically, ganglion cell specific marker MAP1a (Tucker and Matus 1988; Okabe et al. 1989), or cholinergic amacrine cell marker ChAT (Eckenstein and Thoenen 1982; Tumosa et al. 1984; Schmidt et al. 1985; Pourcho and Osman 1986; Tumosa and Stell 1986; Voigt 1986), in combination with CPEB3. Confocal images indicate that most CPEB3-postive cells in RGC layer are ganglion cells (figure 33), and a few are displaced amacrine cells (figure 34).

Both the inner plexiform layer and outer plexiform layer appeared to be immunopositive for CPEB3 labeling (figure 33,34 ). The pattern of CPEB3 in the IPL was diffuse compared to the more laminar pattern of CPEB1 in the same layer (figure 22, 23). Within the location of immunogen for CPEB1 antibody (167-184 a.a.) and that of CPEB3 antibody (between 300-400 a.a.), little homology between CPEB1 and CPEB3 was found. Therefore, cross-reactivity between the two antibodies seemed highly unlikely. The identity of synaptic structures in the plexiform layers was not determined. An extensive characterization with double and triple labeling methods was beyond the scope of this dissertation. 
Figure 33. Localization of CPEB3 protein- with MAP1a

Double-immunofluroscence of CPEB3 and MAP1a on P60 mouse retina indicated that the majority of CPEB3 positive cells in the retinal ganglion cell (RGC) layer were also MAP1a positive. MAP1a has been demonstrated as a marker for retinal ganglion cells. The absence of labeling when CPEB3 antibody was pre-adsorbed with recombinant CPEB3 protein (right upper panel) demonstrated the specificity of the CPEB3 antibody. Images were acquired with the aid of an Olympus confocal microscope. The scale bar represented $50 \mathrm{um}$. 
Figure 33
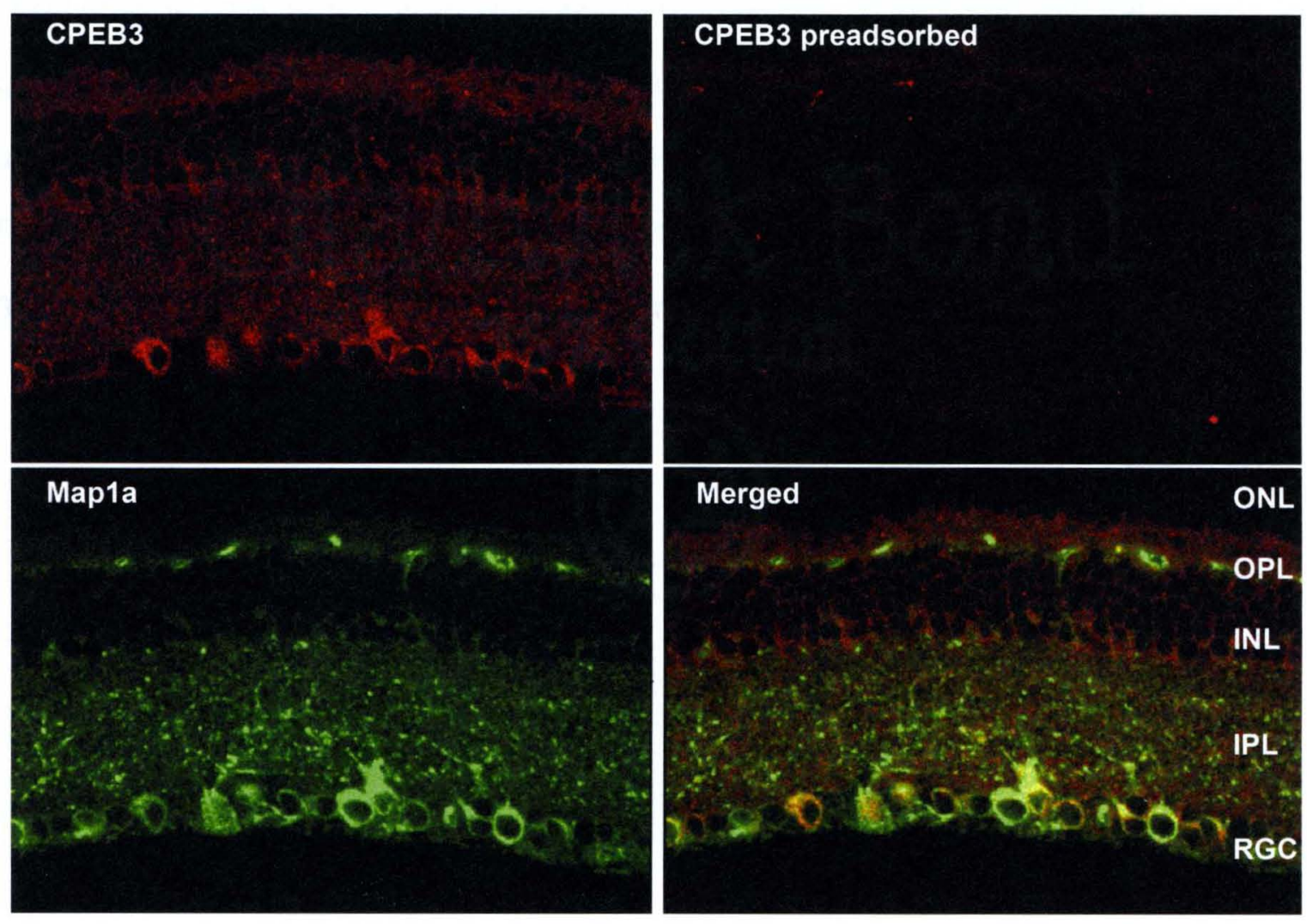
Figure 34. Localization of CPEB3 protein- with ChAT

Double-immunofluorescence of CPEB3 and ChAT on p60 mouse retina indicated that a few CPEB3 positive cells in RGC layer were also ChAT positive, as indicated with the arrows. ChAT is a marker for cholinergic amacrine cells and displaced amacrine cells. This suggested that a few CPEB3 positive cells in RGC layer were displaced amacrine cells. The size of such cells was usually smaller than those staining positive for both CPEB3 and Map1a in the RGC layer. Scale bar represented 50um. 
Figure 34
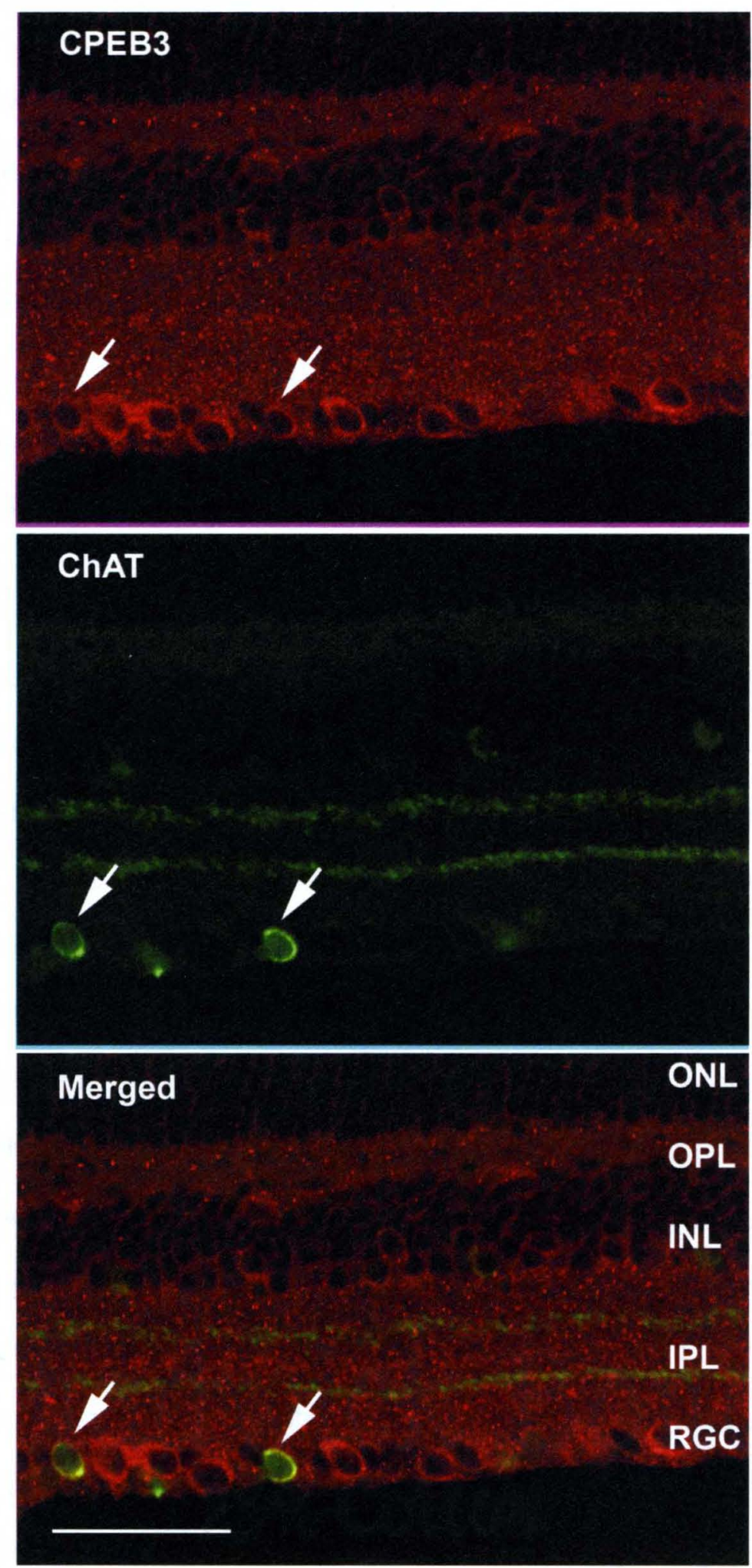
7) Developmental regulation of CPEB3 protein: CPEB3 protein is up-regulated during postnatal development of the retina

Our next aim was to investigate the relative abundance of CPEB3 protein in the developing retina with the aid of Western blots. To examine antibody specificity we used a recombinant CPEB3 protein to pre-adsorb the antibody. This served as a negative control. The GST-CPEB3 fusion protein induced in E. coli was visualized on SDS-PAGE gel (figure 35a). The induced protein was then purified by immunoprecipitation, and subjected to mass spectrometry for identification. MS results (figure 35b) confirmed its identity as CPEB3. The fusion protein was then used as a blocking reagent for the CPEB3 antibody.

We identified four immunopositive bands in Western blots that were diminished by preadsorption (figure 35c). These included bands with relative molecular weight of $\sim 130 \mathrm{kD}$, $75 \mathrm{kD}, 67 \mathrm{kD}$ and $62 \mathrm{kD}$. The $75 \mathrm{kD}$ band appeared to be the mature form based on its predicted size in the database. Our results indicated that this band increased significantly in intensity during postnatal development of the retina. This was consistent with data demonstrating a postnatal increase in CPEB3 mRNA (figure 32). The other bands may represent isoforms from other transcript variants present in the retina, breakdown products, or other CPEB3-related products. One band ( 110kD) appeared not to be blocked by the pre-adsorption of antibody with fusion protein. The unblocked band may be a problem when the same antibody was used for immunohistochemistry; however this issue was resolved by using pre-adsorbed antibody as a negative control for the immunocytochemistry, which showed no signal. Statistical analysis (ANNOVA T tests) indicated that there was significant increase in the level of CPEB3 (75kD) from P1 and P7 to P12 (figure 35d). 
Figure 35. Developmental pattern of CPEB3 proteins

CPEB3 protein was up-regulated during the postnatal development of the retina.

a) The expression of recombinant CPEB3 protein. An extra band of the expected size for GST-CPEB3 fusion protein appeared in IPTG- induced sample (+) compared to non-induced sample (-).

b) The extra band in samples induced for recombinant CPEB3 was cut out and subjected to mass spectrometry. The pattern from MS confirmed that this band was recombinant CPEB3 protein.

c) CPEB3 western blot on retinal samples from mice of different postnatal ages. The results showed that there was an increase in the amount of CPEB3 protein during the development ( $75 \mathrm{kD}$ band). This band disappeared when the antibody was pre-adsorbed with the recombinant CPEB3 antibody (the two lanes to the right). Another two bands at $\sim 130 \mathrm{kD}$ and $\sim 65 \mathrm{kD}$ respectively also disappeared when CPEB3 antibody was pre-adsorbed with recombinant CPEB3 proteins, suggesting that they represent different forms of CPEB3. The up-regulation of CPEB3 protein during development is consistent with that of CPEB3 mRNA. GAPDH was used as a loading control.

d) Quantification of the level of CPEB3 protein during development. Significance up-regulation was observed between two bracketed age set: P1 to P12, and P7 to P12 ( $p<=0.05)$. Each CPEB3 band was normalized to the GAPDH band in the same sample. Annova was used for statistics on three biological repeats. Error bars indicated standard deviation. 
Figure 35

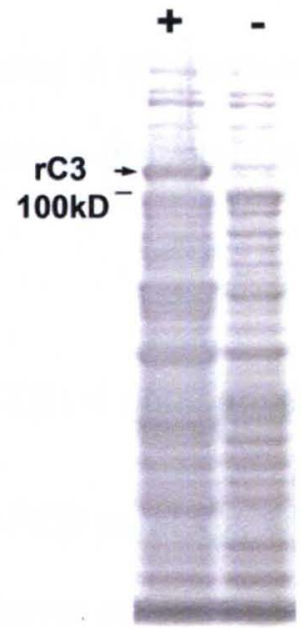

a

P1 P7 P12 P14 P16 P30 P60 P7 P60

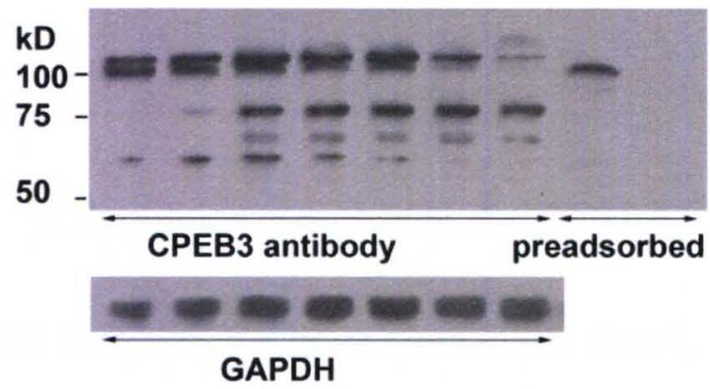

C

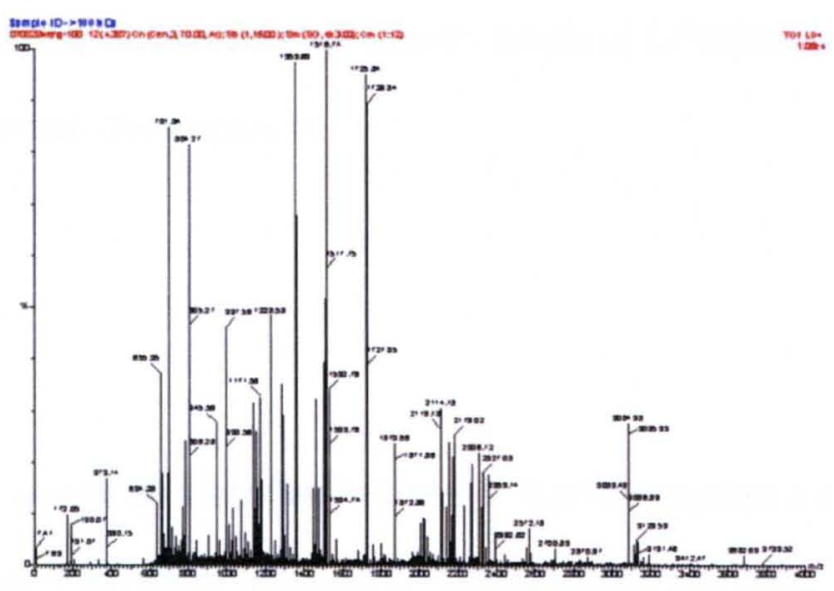

b

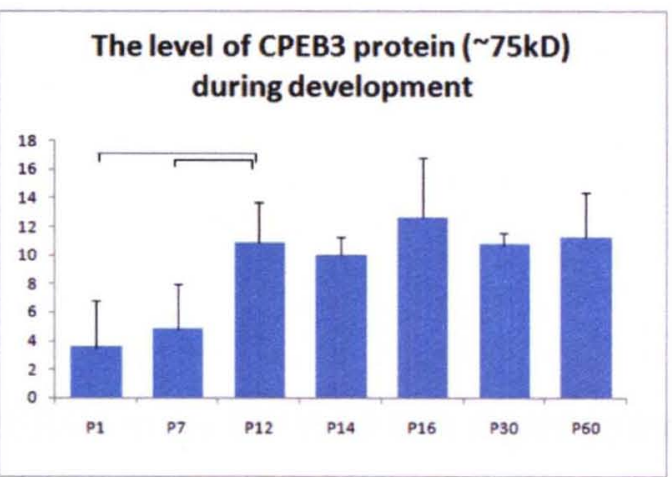

d 
In summary, our study identified several CPEB3 isoforms (both mRNA and protein) from the NCBI and Swiss-Prot/TrEMBL databases. A novel CPEB3 isoform was identified experimentally for the first time. Among the previously known isoforms and the isoforms we identified, only one had discriminative tissue distribution. Our data also demonstrated the presence of CPEB3 in the retina. CPEB3 was predominantly expressed in the inner retina. Both the mRNA and the protein levels of CPEB3 were upregulated in the retina during postnatal development.

3. CPEB1-4 comparisons

Four CPEB paralogs, CPEB1-4, have been identified in rodent. Current studies on cytoplasmic polyadenylation are predominantly focused on CPEB1, whereas the mechanisms of CPEB1 are yet to be fully established. Very little is known with regard to CPEB2-4. We were able to draw some comparison among the four CPEBs based on our study within the retina.

1) Sequence comparison of CPEB1-4

Some comparisons have been made among CPEB1-4 in the literature with regard to the RNA recognition domain (Theis et al 2003). In our study we performed a comprehensive sequence alignment of those four. Since CPEB1 is more distant to CPEB2-4 in the family tree, therefore, we first aligned CPEB1-4 (figure 36). Then, in order to better reveal the differences and similarities between CPEB2-4, we aligned these three separately (figure 37). 
Figure 36. Sequence alignment of CPEB1-4 proteins

Sequence alignment was performed using ClustalW. Sequences with the following accession numbers were used: CPEB1: NP_031781.1; CPEB2: NP_787951.1; CPEB4: NP_938042.2; CPEB4: NP_080528.2. The alignment demonstrated higher homology in the $\mathrm{C}$-terminus compared to the $\mathrm{N}$-terminus. CPEB1 had more variance from CPEB2-4 across the whole sequence. Asterisk represented 100\% identity; colons represented substitution with a similar amino acid; period represents substitution with a different amino acid. 
Figure 36 (to be continued)

CPEB2

CPEB 3

CPEB4

CPEB 1

CPEB2

CPEB 3

CPEB4

CPEB 1

CPEB2

CPEB 3

CPEB4

CPEBI

CPEB2

CPEB 3

CPEB4

CPEB 1

CPEB2

CPEB3

CPEB 4

CPEB 1

CPEB 2

CPEB3

CPEB 4

CPEB1

MDKSKT- ANGSSAGSAWLFPAPATHNIQDEILGSEKAKSQQQEQQDPLEKQQLSPSP 100

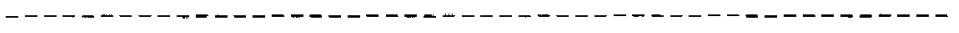

PLSSEIPKPEDSSAVPALSPASAPPAPNGPDKMQMESPLLPGLSFHQPPQ 92 GQEAGILPETEKAKAEENPGDSSSENSNGKEKLRIESPVLTGFDYQEATG 150

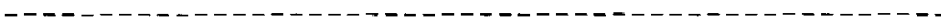

QPPPPQEPTAPGASLS----PSFGSTWSTGTTNAVEDSFFQG-----ITP 133 LGTSTQPLTSSASSLTGFSNWSAAIAPSSSTIINEDASFFHQGGVPGASA 200 .

CPEB 2

CPEB3

CPEB4

CPEB1

MNLPQ----QQPPAAAPQQPQSRRS 21

VNGTMLFQNFPHHVNPVFGGTFSPQIGLAQTQHHQQPPPPAPQPPQPAQP 183 NNGALLFQNFPHHVSPGFGGSFSPQIGPLS-------QHHPHHPH-FQH 241 CSNANIFRRINAILDDSLDFSKVCTTPINRG-----IHDQLPDFQDSEET 71

PVSPQLQQQHQAAA---AAFLQQRNSYNHHQPLLK--QSP---------- 56 PQAQPSOQRRSPASPSOAPYAORSAAAYGHOPIMT--SKPSSSSAVAAAA 231 HHSOHQQQRRSPASPHPPPFTHRSAAFNQLPHLANNLNKPPSP- - - - - 284 VTSRMLFPTSAQESPRGLPDANGLCLGLQSLSLTG-WDRP_....... 110

$$
\text { : }: \text { : : } \quad \text { : * }
$$

----------WSNHQ----- NSGWGTASMSWG--AMHG-RDHRRSG-- 84

AAAAASSASSSWNTHOSV----NAAWSAPSNPWG--GLQAGRDPRRAVGV 275 - - - - - - WSSYQSPSPTPSSSWSPGGGGYGGWGASQGRDHRRGLN- 322 $\stackrel{*}{*}$ :

CPEB2

CPEB3

CPEB4

CPEB1

----NMGIPGTMNQISPLKKPFSGNVIAPPKFTRSTPSLTPKSWIEDNVF 130 GVGVGVGVPSPLNPISPLKKPFSSNVIAPPKFPRAAP-LTSKSWMEDNAF 324 -----GGITPLNSISPLKKNFASNHIQLQKYARPSSAFAPKSWMEDSLN 366 -----LDPLGSDLDKFPAPSVRGSRLDTRPILDSRSSSPSDSDTSGFSS 192 
Figure 36 (continued)

CPEB2

CPEB 3

CPEB4

CPEB1

CPEB2

CPEB3

CPEB 4

CPEB1

CPEB2

CPEB3

CPEB 4

CPEB1

CPEB2

CPEB3

CPEB 4

CPEB1

CPEB 2

CPEB 3

CPEB 4

CPEB1

CPEB2

CPEB 3

CPEB4

CPEB1

CPEB2

CPEB 3

CPEB4

CPEB1

CPEB2

CPEB 3

CPEB4

CPEB 1

CPEB2

CPEB3

CPEB 4

CPEB1

RTDNNSNTLLPLQVRSSLQLPAWGSDSLQDSWCTAAGTSRIDQDRSRMYD 180 RTDN-GNNLLPFQDRS-RPYDTFNLHSLENSLMDMIRTD-HEPLKGKHYP 371 RADN----IFPFPERP----RTFDMHSLES-GSDHLSDLISSLRISPPLPFLSMTGNGPRDPLKMGVGSR-------- 231 $: *:$ : : . : . .

SLNMHSLENSLIDIMRAEHDPLKGRLSYPHPGTDNLLMLN--------GR 222 PS---GPPMSFADIMWRNHFAGRMGINFHHPGTDNIMALNTRSYGRRRGR 418 --- - - MDQEQAALAAVAPSPTSAPKRWPGASWWPSWDLLG------- 266 . : .

SSLFPIDDSLLDDGHSDQVGVLNSPTCYS--AHQNGERIERFSRKVFVGG 270 SSLFPFEDAFLDDSHGDQA-LSSGLSSPT--RCQNGERVERYSRKVFVGG 465 SSLFPMEDGFLDDGRGDQP-LHSGLGSPHCFTHQNGERVERYSRKVFVGG 478 APKDPFSIEREARLHRQAAAVNEATCTWSGQLPPRNYKNPIYSCKVFLGG 316

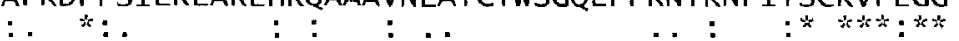

LPPDIDEDEITASFRRFGPLWDWPHKAESKSYFPPKG-----YAFLLFQ 315 LPPDIDEDEITASFRRFGPLVVDWPHKAESKSYFPPKG----YAFLLFQ 510 LPPDIDEDEITASFRRFGPLIVDWPHKAESKSYFPPKG----YYALLFQ 523 VPWDITEAGLVNTFRVFGSLSVEWPGKDGKHPRCPPKGNMPKGYVYLVFE 366 $: * * * *: .: * * * * * *: * * * \quad: . * * * * \quad * * * *:$

EESSVQALIDACIEE-----DGKLYLCVSSPTIKDKPVQIRPWNLSDSD 359 EESSVQALIDACLEE-----DGKLYLCVSSPTIKDKPVQIRPWNLSDSD 554 DESSVQALIDACIEE------DGKLYLCVSSPTIKDKPVQIRPWNLSDSD 567 LEKSVRALLQACSHDPLSPDGLSEYYFKMSSRRMRCKEVQVIPWVLADSN 416

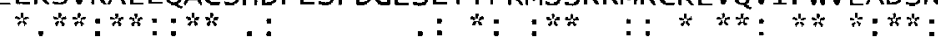

FVMDGSOPLDPRKTIFVGGVPRPLRAVELAMIMDRLYGGVCYAGIDTDPE 409 FVMDGSQPLDPRKTIFVGGVPRPLRAVELAMIMDRLYGGVCYAGIDTDPE 604 FVMDGSQPLDPRKTIFVGGVPRPLRAVELAMIMDRLYGGVCYAGIDTDPE 617 FVWSPSQRLDPSRTVFVGALHGMLNAEALAAILNDLFGGVVYAGIDTD-K 465

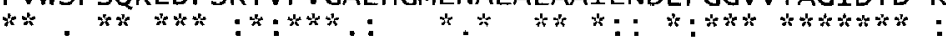

LKYPKGAGRVAFSNOQSYIAAISARFVOLOHGDIDKRVEVKPYVLDDOMC 459 LKYPKGAGRVAFSNQQSYIAAISARFVQLQHNDIDKRVEVKPYVLDDQMC 654 LKYPKGAGRVAFSNQQSYIAAISARFVQLQHGEIDKRVEVKPYVLDDQLC 667 HKYPIGSGRVTFNNQRSYLKAVTAAFVEIKTTKFTKKVQIDPY-LEDSLC 514

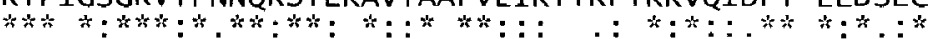

DECQGARCGGKFAPFFCANVTCLQYYCEFCWANIHSRAGREFHKPLVKEG 509 DECQGTRCGGKFAPFFCANVTCLQYYCEYCWASIHSRAGREFHKPLVKEG 704 DECQGARCGGKFAPFFCANVTCLQYYCEYCWAATHSRAGREFHKPLVKEG 717 LICS--- SQPGPFFCRDQVCFKYFCRSCWHWRHSMEGLRHHSPLMRNQ 559

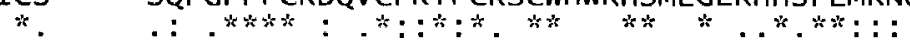

ADRPRQIHFRWN 521

GDRPRHVPFRWS 716

GDRPRHISFRWN 729

KN-D------ 561 
Figure 37. Sequence alignment of CPEB2-4 proteins

Sequence alignment was performed using ClustalW. RefSeq sequences with the following accession numbers were used: NP_787951.1 for CPEB2, NP_938042.2 for CPEB3, and NP_080528.2 for CPEB4. Sequences highlighted in blue were unique to CPEB3, and sequences highlighted in yellow were highly conserved among CPEB2-4. Asterisk indicated $100 \%$ conservation; colon indicated substitution with a similar amino acid; period indicated substitution with a distinct amino acid. 


\section{Figure 37}

MGDYGFGVLVQSNTGNKSAFPVRFHPHLQPPHHHQNATPNPAAFINNNTA 50

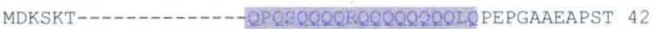
ANGSSAGSAWLFPAPATHNIODEILGSEKAKSOQOEQODPLEKOQLSPSP 100

LSSEI PKPEDSSAVPALSPASAPPAPNGPDKMQMESPLLPGLSFHQPPQ 92 GQEAGI LPETEKAKAEENPGDSSSENSNGKEKLRIESPVLTGFDYQEATG 150

QPPPPQEPTAPGASLS----PSFGSTWSTGTTNAVEDSEFQG-----ITP 133 LGTSTOPLTSSASSLTGESNWSAAIAPSSSTIINEDASFFHOGGVPGASA 200

WNGTMLFONF PHHVNPVFGGTFSPQIGLAQTQHHQQPPPPAPQPPQPAOP 18 NNGALLFQNF PHHVSPGFGGSFSPQIGPLS----OQHHPHHPH-FQH 24$$
{ }^{*}:{ }^{*}:
$$

PQAQPSQQRRSPASPSQAPYAQRSAAAYGHQPIMT--SKPSSSSAVAMAX 231

PVSPQLQQQHQAAA---AAFLQQRNSYNHHQPLLK--QSP--------- 56

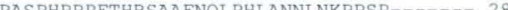

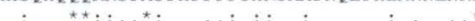

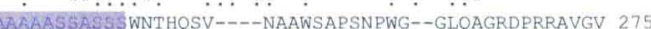
-1-1--WSNHQ----NSGWGTASMSWG--AMHG-RDHRRSG-- 84 -WSSYQSPSPTPSSSWSPGGGGYGGWGASQGRDHRRGLN- 322 t. : * $\therefore{ }^{\star}+. \quad:$ * * **.

GVGVVGVPSPLNPISPLKKPESSNVIAPPKFPRAAP-LTSKSWMEDNAF 324 ---NMGIPGTMNQ ISPLKKPESGNVIAPPKFTRSTPSLTPKSWIEDNVF 130 - - GGITPLNS ISPLKKNEASNHIQLQKYARPSSAFAPKSWMEDSLN 366

RTDN-GNNLLPFODRS-RPYDTFNLHSLFNSLMDMIRTD-HEPLKGKHXX 371 RTDNNSNTLLPLOVRSSLOLPAWGSDSLODSWCTAAGTSRTDODRSRMYD 180

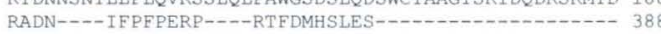
${ }^{\star}::^{\star \star} \quad::^{\star}: \quad * \quad: \quad . . .^{\star \star}:$ LNMHSLENS LIDIMRAEHDPLKGRLSYPHPGTDNLIMLN-------GR 22 - SLIDIMRAENDSIKGRLNYSYPGSDSSLLINARTYGRRRQ 429

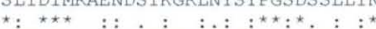

SSLFPFEDAFLDDSHGDQA-LSSGLSSPT--RCQNGERVERYSRKVEVGG 465 SSIFPIDDSLLDDGHSDQVGVLNSPTCYS--AHQNGERTERFSRKVFVGG 270 SSLFPMEDGFLDDGRGDQP-LHSGLGSPHCFTHQNGERVERYSRKVEVGG 478

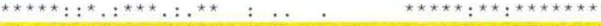
LPPDIDEDEITASFRRFGPLVVDWPHKAESKSYFPPKGYAFLLFQEESSV 320 LPPDIDEDEITASFRRFGPLIVDWPHKAESKSYFPPKGYAFLLFQDESSV 52 OAt QALIDACIEEDGKLYLCVSSPTIKDKPVQIRPWNLSDSDFVMDGSQPLDP 370 QALIDACIEEDGKLYLCVSSPTIKDKPVQIRPWNLSDSDEVMDGSQPLDP 578

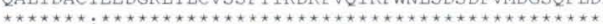
RKTIFVGGVPRPLRAVELAMIMDRLYGGVCYAGIDTDPELKYPKGAGRVA 615 RKT I FVGGVPRPLRAVELAMIMDRLYGGVCYAGIDTDPELKYPKGAGRVA 420 RKTIFVGGVPRPLRAVELAMIMDRLYGGVCYAGIDTDPELKYPKGAGRVA 628 FSINQQSYIAAISAREVQLQHNDIDKRVEVKPYVLDDQMCDECQGTRCGGK 665 FSNQQSYIAAISAREVQLOHGDIDKRVEVKPYVLDDQMCDECQGARCGGK 470 FSNQQSYIAAISARFVQLQHGEIDKRVEVKPYVLDDQLCDECQGARCGGK 678

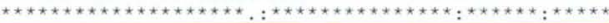
FAPEFCANVTCLQYYCEYCWAS IHSPAGREFHKPLVKEGGDRPRHVPERW 715 APFFCANVTCLOYYCEFCWANI HSRAGREFHKPLVKEGADRPROIHFRW 520 APFFCANVTCLQYYCEYCWAAIHSRAGREFHKPLVKEGGDRPRHISFRW 728

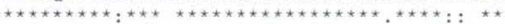

Poly-Q track

Phosphorylation site/ SA track

23 a.a.

8 a.a. (Phospho-site for CaMKIIa/Sp6

The second RRF 
The comparison clearly demonstrated that the $\mathrm{N}$ terminus is more diverse among the different paralogs. The C-terminus, where the important functional domain such as RNA-recognition motifs reside, is highly conserved. The $\mathrm{C}$-terminus is almost identical for CPEB2-4 (95\% identity according to figure 10); however, it varies significantly from the terminus of CPEB1 (45\% identity according to figure 10 ).

2) All four CPEBs are present in the retina

With the aid of regular RT-PCR, we explored the presence of four CPEB paralogs in the retina (figure 38). Total retinal RNA from adult mice was used for the amplification. The primer set for each gene was designed to be exclusive of each other. Amplicons were separated on agarose gel. Distinct bands were cut out and sequenced to confirm the identity. The result demonstrated the all four CPEB mRNA are expressed in the retina. Due to possible differences in amplification efficiency of each primer set, this data is not suitable for quantitative studies, which will be obtained later using real-time PCR experiments (see below).

\section{3) Cellular localization of CPEB1-4 mRNA}

With the aid of in situ hybridization, we compared the tissue distribution of four CPEBs in the retina (figure 39). Each probe was specifically designed with no significant similarity to others. The sequences of the template for each probe were confirmed with sequencing analysis. In situ hybridization demonstrated a similar pattern of four CPEBs: predominantly expressed in the retinal ganglion cell layer; to some extent, to the inner layers of the inner nuclear layer. Some signal appeared to be in the inner plexiform 
Figure 38. Presence of four CPEBs in the retina

P60 mouse retinal total RNA was used for the RT-PCR. $\beta$-actin was used as a positive control. The "-" control had all the components as $\beta$-actin reaction except that the reverse transcriptase was omitted at the RT step. The primer sets for all genes were designed with low homology with each other or with any other genes. Each band in the agarose gel was cut out, purified and sequenced to confirm its identity. The results showed that all four CPEB homologs were expressed in the retina. This result was not suitable for quantification, since each set of primers may have distinct amplification efficiency. Quantification analysis would be inferred from real-time PCR data in later sections. 
Figure 38

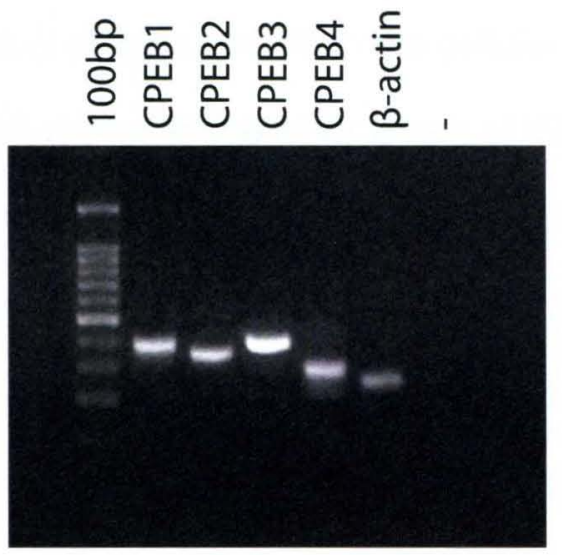


Figure 39. Localization of four CPEB mRNAs in the retina

P60 slides were used for in situ hybridization. Probes for each gene were designed within a region that was less homologous to the other homologs or any other genes. The sequences of all probes were confirmed via sequencing. The results demonstrated that CPEB1-4 have a similar pattern of distribution within the cellular layers of the retina: predominantly in RGC layer, to some extent, in the inner layers of INL. Their distribution in synaptic structures in the plexiform layers could not be confirmed at this point due to the limitation of the resolution of this technique. This result was not suitable for quantification analysis due to the fact that different probes may have distinct binding efficiencies to their complementary mRNA. Quantitative studies would be done based on real-time PCR data. 
Figure 39

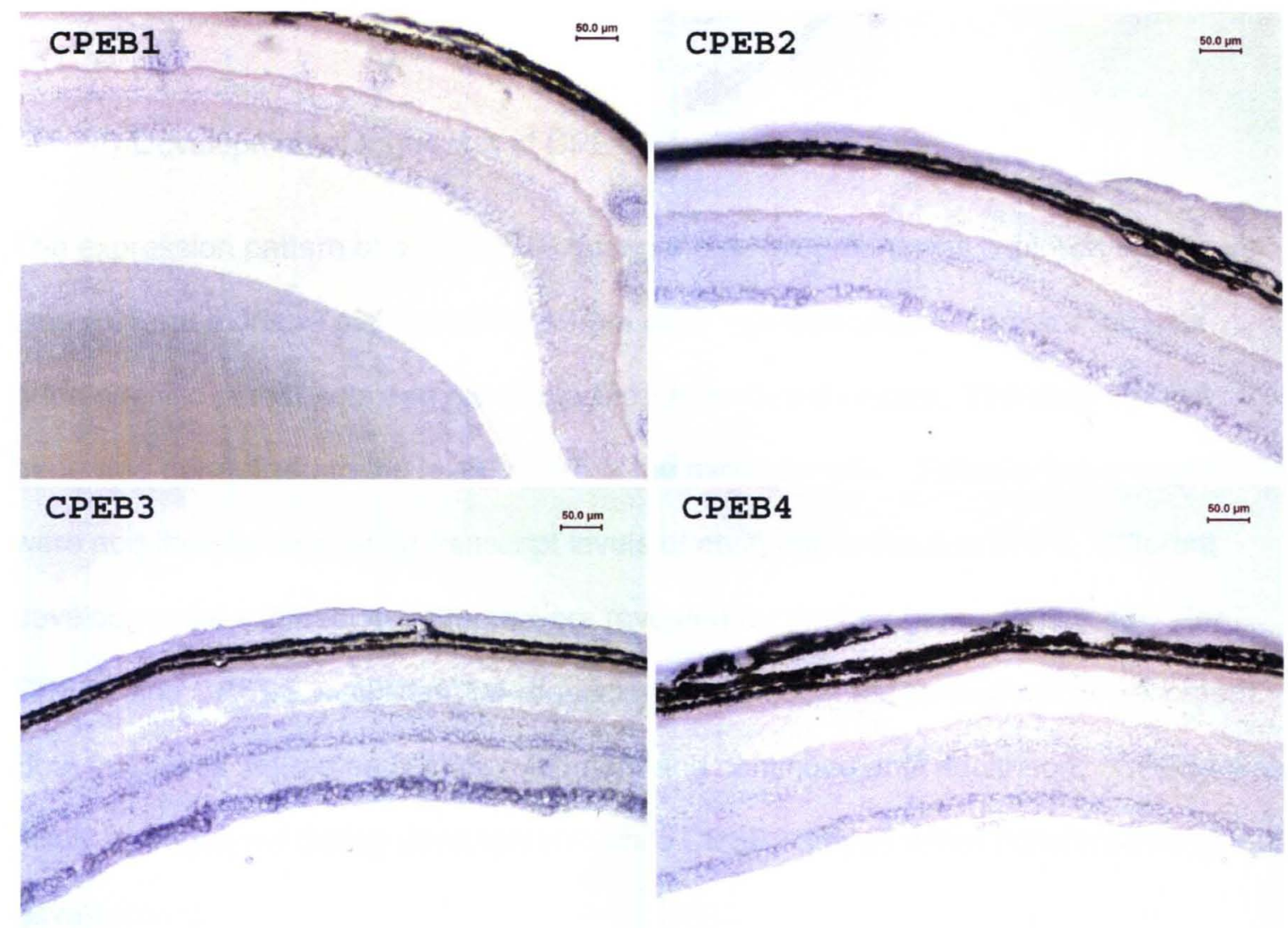


layers, although the fine synaptic structures were not distinguishable due to the resolution of this technique. This data is not suitable for quantitative comparisons since different probes may have different efficiencies for hybridization. Quantitative analysis will be done based on real-time PCR data.

4) Developmental regulation of CPEB1-4

The expression pattern of each CPEB during the development was explored using realtime PCR methods. Total retinal RNA from each age was used in combination with gene-specific primer sets and gene-specific FAM-labeled probes. The level of each gene was normalized to the level of $18 S$ in the same sample. Relative fold changes were acquired by calibrating transcript levels of each age to the age of P1. Different developmental expression patterns were revealed for the four genes (figure 40): For CPEB1 and CPEB3, significant up-regulations were observed throughout development. Both increases started early in development and continued until adulthood. CPEB4 was mildly up-regulated during development; while CPEB2 stayed rather constant throughout development.

For CPEB1 and CPEB3, the mRNA in P12 was increased significantly compared to $\mathrm{P} 1$ or $\mathrm{P} 7$. The level of mRNAs between $\mathrm{P} 12, \mathrm{P} 14$ and $\mathrm{P} 16$ was more like a plateau, with no significant increase between those three ages. Another significant increase occurred in P30, compared to P14 or P16 for CPEB1 and CPEB3, respectively. For CPEB4, a significant increase was found in $\mathrm{P} 12$ compared to $\mathrm{P} 1$; no significant changes were found between P12, P14 and P16; the level of CPEB4 was increased significantly further in adult (P60) compared to P12. The overall increases in the level of transcripts of 
Figure 40. Four CPEBs - trends in development

Total RNA from the retinas of postnatal day1, $7,12,14,16,30$, and 60 mice which were collected at the same time of the day was used for real-time PCR assays. The combination of gene-specific primers and FAM-labeled gene-specific probes ensured high specificity. $18 \mathrm{~S}$ was used as an endogenous control. The relative fold changes were acquired by calibrating all the older ages to age P1. Each age had 6-10 animals. The Anova procedure, Tukey's Studentized Range (HSD) was used for statistics. Error bars indicated standard deviation (SD). Significant changes were demonstrated between each two bracketed ages $(p<=0.05)$. The results demonstrated significant increases of CPEB1 and CPEB3 mRNA during post-natal development. CPEB4 had a slow increase; while CPEB2 stayed constant. 

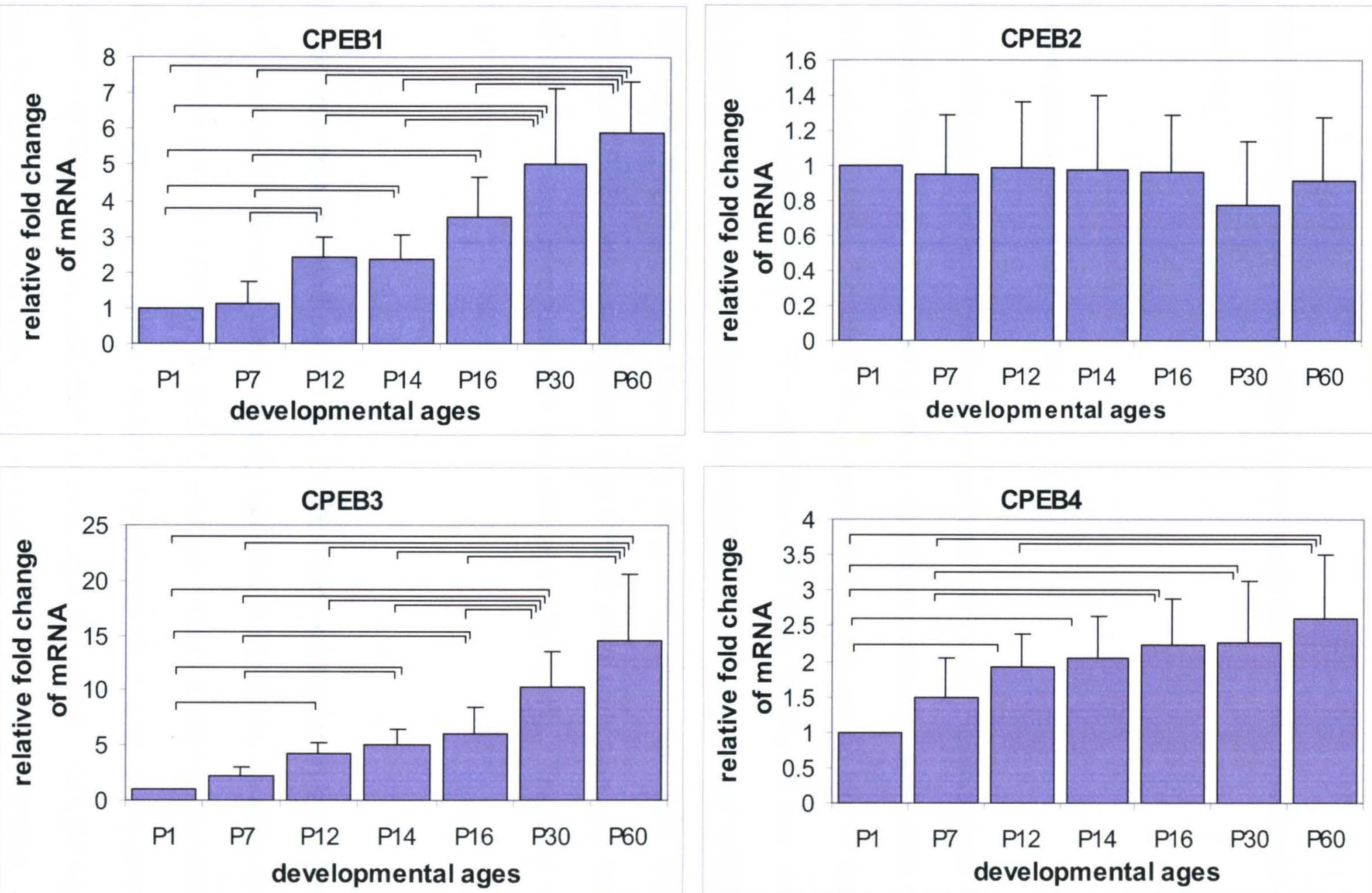
CPEB1, CPEB3, and CPEB4 from the newborns to the adults were 6-fold, 13-fold, and 2.5 -fold respectively.

5) Relative abundance of four CPEBs

Comparisons across genes were derived from the real-time PCR data. Relative levels of mRNA for different genes were acquired by simply normalizing levels of each gene to that of $18 \mathrm{~S}$ in the same sample. Strikingly, the most abundant gene among four CPEBs was not the overtly regulated ones. The most abundant gene among all four was CPEB4. The level of CPEB3 was the second most abundant. Both CPEB3 and CPEB4 were more than one magnitude, sometimes two magnitudes more abundant than CPEB1 and CPEB2. CPEB1 was less abundant than CPEB2 in the early ages, but then surpassed CPEB2 as it was up-regulated in the later ages (figure 41). The level of CPEB2 was consistently low.

In summary, our data demonstrated that all four CPEBs were expressed in the retina. The mRNAs of all four CPEBs were predominantly localized in the retinal ganglion cell layer. The transcription of CPEB1, 3 and 4 was up-regulated during postnatal development; while CPEB2 stayed rather constant. The relative abundance at any given age was CPEB4 > CPEB3 > CPEB2 or CPEB1. In early ages (P1 and P7), CPEB2 was more abundant than CPEB1, in older ages (P30 and P60), CPEB1 surpassed CPEB2. 
Figure 41. Comparison of the relative abundance of four CPEBs

Total RNA from the retinas of postnatal day1, $7,12,14,16,30$, and 60 mice which were collected at the same time of the day was used for real-time PCR assays. The combination of gene-specific primers and FAM-labeled gene-specific probes ensured high specificity. $18 \mathrm{~S}$ was used as an endogenous control. The relative fold changes were acquired by calibrating all the older ages to age P1. Each age had 6-10 animals. The Anova procedure, Turkey's Studentized Range (HSD) was used for statistics. Error bars indicated standard deviation (SD). Significant differences were indicated between each two bracketed ages $(p<=0.05)$. The results demonstrated that of any given age, CPEB4 mRNA was the most abundant; CPEB3 was the second most abundant. CPEB1 mRNA was the lowest, but because of its up-regulation during the development, it surpassed CPEB2, which stayed constant, in older animals. 
Figure 41

Relative abundance of CPEBs in developing retina

$\vec{\sigma}$

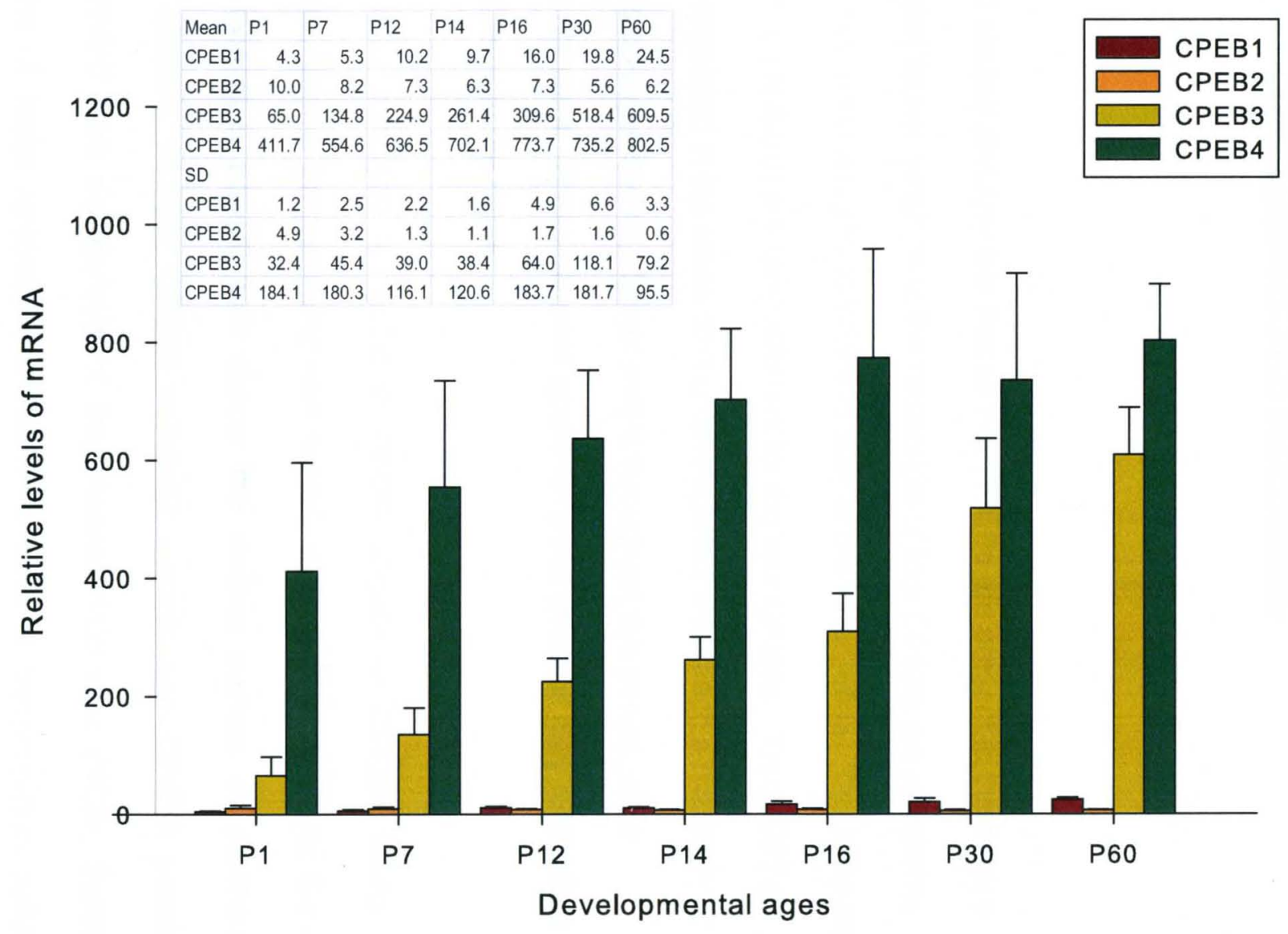




\section{CHAPTER V \\ CONCLUSIONS AND DISCUSSION}

In conclusion, these studies demonstrate that all four CPEBs are present in the mouse retina. The spatial distribution patterns of the transcripts of four CPEBs are somewhat different but all four are predominantly localized in cells of the inner retina. The temporal expression patterns, in particular are quite different for the four CPEBs. Thus, CPEB1 and CPEB3 are up-regulated in the retina during development and reach a maximum in adulthood; CPEB4 is up-regulated to a lesser extent throughout this period; while CPEB2 stays rather constant. These different spatio-temporal patterns may reflect their as yet undiscovered roles in the retina.

In this study we also screened for potential mRNA targets of CPEB1, using a combination of computational programming and wet-lab experiments. Some of these mRNAs acquired elongated poly(A) tails during the earliest periods of postnatal development. In addition, multiple CPEB3 transcript variants and СРEB3 protein isoforms were identified from an analysis of the national databases (NCBI, SwissProt/TrEMBL). One of these isoforms was found to have a discriminative tissue distribution. Additionally, a novel transcript of CPEB3 was identified through these studies. This new isoform which lacks exon11 is present in all tissues tested including the retina. 


\section{DISCUSSION}

1. Distinct expression patterns and sequence discrepancies indicate functional divergences between members of the CPEB family

The expression patterns of four CPEBs in the retina demonstrated some uniqueness for each paralog. Although in situ hybridization data showed similar patterns of the four, localization data from immunohistochemistry microscopy refined the differences between the patterns of CPEB1 and CPEB3 in the inner plexiform layer. CPEB1 had a laminar structure, while CPEB3 was more diffusive. Developmental studies further compared the regulation of four CPEBs postnatally. From newborn to adult, CPEB1 and CPEB3 was up-regulated 6-13 fold, respectively; CPEB4 increased by about 1.5 fold, whereas CPEB2 stayed constant. Both the spatial and temporal patterns of CPEBs in the retina revealed some degree of diversity. Similarly, distinct expression patterns of CPEB1-4 were also reported in the hippocampus (Theis et al. 2003; Huang et al. 2006).

Sequence comparisons demonstrated that CPEB1 is distant from CPEB2, 3 and 4 . The highly conservative C- terminus of CPEB1 had merely $45 \%$ identity to the other three. The RNA recognition motifs between CPEB2, 4 and the prototypical CPEB3 are 97-99\% identical, indicating high degree of overlapping with respect to substrate specificity. However, their $\mathrm{N}$-termini where regulational domains such as phosphorylation sites reside are dramatically variable.

The accumulative evidence on the structure, distribution, and regulation of CPEBs may dictate their functional distinctness. Such differences may embody multiple aspects 
such as spatial and temporal availability, substrate specificity, responses to discrete stimuli, and signaling cascades they are associated with.

With those notions made, four CPEBs share some common features both structurally and functionally. Current knowledge on CPEB1, which has been studied most extensively, may be applicable to speculate on CPEB2-4 when appropriate to broaden our view.

2. CPEBs and the inner retina

1) CPEBs are expressed in the RGC layer, the INL, and the IPL.

All four CPEBs are present in the inner retina. In situ hybridization indicated that transcripts of all four CPEBs are predominantly expressed in the retinal ganglion cell layer, and to some extent, in the inner nuclear layer. Immunocytochemistry with available antibodies to CPEB1 and CPEB3 showed positive lableling in retinal ganglion cells and amacrine cells. Only a subset of ganglion cells are positive for CPEB1 or CPEB3, since not all MAP1 positive cells are CPEB1 positive or CPEB3 positive. Similarly, only a small population of cholinergic amacrine cells is CPEB3 or CPEB1 positive.

Immunofluorescence labeling of fixed tissue sections also strongly demonstrated the presence of CPEB1 and CPEB3 within the inner plexiform layer. CPEB1 labeling was associated with laminar structures in the inner plexiform layer, whereas CPEB3 labeling demonstrated a more diffuse pattern. The CPEB1 immunopositive laminae did not appear to co-localize with ChAT immunolabeling. The close juxtaposition of the CPEB1 
positive laminae to ChAT positive laminae suggested that CPEB1 laminae may reside in sublamina a and sublamina $b$ of the IPL.

2) The identities of the CPEB positive cells and/or synapses need to be further characterized.

The subtypes of the CPEB1 or CPEB3 positive ganglion cells were not specifically identified in our study but should be pursued in future studies. About 11 morphologically distinct subtypes of RGCs with distinct dendritic architectures are known to be present in the mammalian retina (Cajal 1893; Yamagata and Sanes 1995a; Yamagata and Sanes 1995b; Rodieck 1998; Rockhill et al. 2002). More than a dozen of subtypes of amacrine cells have been described (Kolb 1997) based on the diameter of their dendritic field and the sublamina where their synapses are located. The number of types of amacrine cells continues to grow as techniques such as intracellular physiological recording, immunohistochemistry, and biochemical methods became available (Casini et al. 1995; Crooks and Kolb 1992; Kolb et al. 1992; MacNeil and Masland 1998). Immunohistochemistry combining CPEB antibody with the subtype-specific antibodies can help to pinpoint the identity of the CPEB positive cells.

Similarly, the identity of the CPEB positive structures in the inner plexiform layer can be addressed. The IPL was divided into five equal-thick strata (Cajal 1892) into which synapses between bipolar, amacrine and ganglion cells can be assigned. A sublaminar division was later applied to the IPL in superimposition to Cajal's five strata (Famiglietti and Kolb 1976): sublamina a corresponds to strata 1-2; sublamina b corresponds to strata 3-5. Distinct subtypes of RGCs maintain their functional specificity by forming synaptic connections with appropriate pre-synaptic bipolar cells or amacrine cells at 
distinct sublaminae of the IPL. Many amacrine cells make their contacts with RGCs in a single stratum in the IPL, but some are bi-stratified or even tri-stratified (Strettoi et al. 1992; Zucker and Ehinger 1998; Loeliger and Rees 2005; Mojumder et al. 2008). Further characterization need to be done to identify CPEB structures in the IPL with the assistance of cell-type specific markers.

3) The laminar structure of CPEB1 labeling in IPL may be related to ON and OFF pathways

One of the basic principles of the visual signal processing in the visual system is the separation of ON and OFF pathways at virtually all levels of the visual pathway. The ON pathway responds to onset of a light stimulus; while the OFF pathway to the termination of a light stimulus. The separation of ON pathway and OFF pathway starts at the first synaptic contact - the bipolar cell level in cone-driven systems, and at the second synaptic contact - amacrine cell level in rod-driven system. They remain separated at the retinal ganglion cell level, and in upper levels of the visual system such as the lateral geniculate nucleus (LGN) and the visual cortex. Cone ON-bipolar cells are thought to exclusively express metabotropic glutamate receptor 6 (mGluR6), and form synaptic contacts with ON ganglion cells in sublamina b of the IPL; whereas OFF bipolar cells form synaptic contacts with OFF ganglion cells in sublamina a.

At least two laminar structures in the IPL for CPEB1 labeling were distinguishable. They appeared to be non-cholinergic synapses since they did not co-localize with ChAT labeling. But they appeared to be in close proximity of the two ChAT positive laminar structures, which are known to reside at strata 2 (within sublamina a) and strata 4 (within sublamina b) respectively (Kang et al. 2004). It is highly likely that the two CPEB1 
positive lamina structures are located in sublaminar a and sublamina b, respectively, which will make a plausible inference that CPEB1 in these two synaptic bands contribute to OFF pathway and ON pathway.

4) The spatial patterns of CPEBs resemble that of some synaptic molecules which are potential targets of CPEBs

Although the significance of the distribution pattern of CPEBs in the retina is yet to be delineated, we realized that such expression patterns resemble the known expression patterns of some synaptic molecules which are potentially targets of CPEB based on our investigation. Such mRNAs include: CaMKII (Liu et al. 2000), NR1, NR2A-D (Grunder et al. 2000b; Araki and Hamassaki-Britto 2000), GluR1, and GluR2 (Grunder et al. 2000a; Hack et al. 2001; Kamphuis et al. 2003; Dijk and Kamphuis 2004). Immunohistochemistry in combination with in situ hybridization can determine whether these mRNAs co-localize with CPEBs in the retina. This may help to clarify the relationships between these molecules and CPEB.

3. CPEB3 and the outer retina

One of the differences between the spatial patterns of CPEB3 and CPEB1 was that CPEB3 was present in the outer plexiform layer at a more profound level (figure 22, 23, $33,34)$. This may indicate a distinct aspect of the function of CPEB3 in the outer plexiform layer. Although we did not determine whether CPEB3 is localized in the presynaptic or post-synaptic compartment due to the lack of a good post-synaptic marker 
(Aartsen et al. 2006), further investigation on the subcellular location of CPEB3 is necessary for delineating its role in outer plexiform layer.

The outer plexiform layer possesses some degree of plasticity for structural and functional remodeling. Ectopic synaptogenesis was observed in retinas undergoing degeneration. For instance, the retina with mutations for presynaptic cytomatrix protein Bsn, calcium binding protein Cabp4, or the calcium channel protein, Cacna1f, manifested impaired synaptic transmission. Along with the structural and functional defects, horizontal cells and bipolar cells grew their neurites into the outer nuclear layer, an area usually devoid of synapses, and formed synaptic contacts with the photoreceptor cells (Chang et al. 2006; Mansergh et al. 2005; Dick et al. 2003; Haeseleer et al. 2004). Such ectopic synapses were formed de novo, long lasting and capable of sustained neurotransmitter release (Specht et al. 2007). Similar remodeling in the outer plexiform layer has been reported in other types of genetically modified mice with photoreceptor degeneration (Peng et al. 2000; Strettoi and Pignatelli 2000; Claes et al. 2004; Strettoi et al. 2004; Jansen and Sanyal 1992; Peng et al. 2003). It was also observed under pharmacological conditions (Wang et al. 2008) or pathological conditions such as stroke, AMD or normal aging retina (Eliasieh et al. 2007; Sullivan et al. 2007).

The mechanism underling such synaptic plasticity is yet to be unraveled. It is not known whether the retina recapitulates the mechanism from other parts of the CNS, such as the hippocampus and the visual cortex, where CPEB mediated local translation plays a key role in the LTP or LTD. One possible theory is that CPEB3 expressed in the outer plexiform layer is involved in processes related to synaptic activity. Mutant or pathological models can be used to determine whether there is an association of CPEB3 with synaptic remodeling in the outer plexiform layer in the retina. 


\section{CPEB1, CPEB3, CPEB4 and synaptogenesis}

Three CPEBs, CPEB1, 3, and 4 were up-regulated during the postnatal development of the retina. The significant increases occur between $\mathrm{P} 1$ and $\mathrm{P} 12$, as well as between P16 to P60. The increases of these three CPEBs were less profound around eyeopening time (P12 to P16). The enhanced expression of these genes during development, both at transcriptional level and translational level, may be a good indicator for their possible involvement in synaptogenesis.

It is well established that the mammalian retina is premature at birth. Mouse retina reportedly matures on postnatal day 41 (Blanks and Bok 1977). The ganglion cell layer and the inner plexiform layer are recognizable at the time of birth, but the remainder of the retina is yet to be fully developed. The synaptogenesis in the inner plexiform layer (IPL) starts at day 3 and slows down precipitously at day 15 (around the time of eyeopening (Fisher 1979b; Fisher 1979a). Robust glutamatergic synaptic formation occurs in the second postanatal week, and synaptic sublamina in the inner plexiform layer becomes visible on day 12 (Sherry et al. 2003). Synaptic connections in OPL begin to develop on day 3 and are well established by day 12, the time when the first light response was recordable as ERG (Rich et al. 1997). Synaptic contacts between photoreceptors and bipolar cells in the OPL, and between bipolar cells and ganglion cells in the IPL are established slightly before eye opening (Marquardt and Gruss 2002).

The up-regulation of CPEB1, CPEB3, and CPEB4 during the first two weeks is concurrent with the process of synaptogenesis, which prompts the likelihood that CPEBs are either of key significance to the process of synaptogenesis, or their expression is regulated as a consequence of synapse formation. Eye opening may dampen the 
enhanced expression of CPEBs. Future investigations on the interaction of CPEB proteins and synaptic mRNAs may be devoted to synaptic molecules such as GluR2 mRNA, a known target of CPEB3.

AMPAR is known for its key role in fast synaptic transmission. The expression of GluR $2 / 3$ and 4 before the emergence of synaptic transmission indicated a role of AMPAR in the establishment of retinal circuitry (Grunder et al. 2000a; Chang and Chiao 2008). The onset and regulation of GluR2 expression in the retina is in accordance with the process of synaptogenesis: starts early in the postnatal period, and reaches a peak before eyeopening (Johansson et al. 2000; Grunder et al. 2000a). In the inner plexiform layer, the pattern of GluR2 expression changes from a disoriented pattern at birth to a laminated pattern prior to eye-opening (Johansson et al. 2000), a manifestion of a reorganization of synaptic circuits.

Synaptogenesis in early development is activity-dependent (Wong and Ghosh 2002). The formation of synaptic contacts requires the mobility of dendritic structures regulated by spontaneous glutaminergic transmission. The rate of dendritic mobility was decreased by antagonists of both NMDA receptors and non-NMDA glutamate receptors (Wong et al. 2000). In the long term plasticity in the hippocampus and the visual cortex, NMDAR activation results in CaMKIla-mediated delivery of AMPAR to the synaptic membrane for enhanced synaptic efficacy, and CPEB1 mediates rapid local translation of CaMKIla mRNA in active synaptic sites. In the hippocampus, under a different stimulus - kainate induced seizure, CPEB3 regulates the translation of GluR2 mRNA (Huang et al. 2006). If similar machinery is harnessed for synaptogenesis, then one may relate such key molecules to CPEB. The expression pattern for CPEB3 presented here is similar to the pattern for retinal GluR2 (Dijk and Kamphuis 2004; Grunder et al. 2000a; Hack et al. 2001; Hamassaki-Britto et al. 1993; Kamphuis et al. 2003). Both are 
enhanced expression of CPEBs. Future investigations on the interaction of CPEB proteins and synaptic mRNAs may be devoted to synaptic molecules such as GluR2 mRNA, a known target of CPEB3.

AMPAR is known for its key role in fast synaptic transmission. The expression of GluR $2 / 3$ and 4 before the emergence of synaptic transmission indicated a role of AMPAR in the establishment of retinal circuitry (Grunder et al. 2000a; Chang and Chiao 2008). The onset and regulation of GluR2 expression in the retina is in accordance with the process of synaptogenesis: starts early in the postnatal period, and reaches a peak before eyeopening (Johansson et al. 2000; Grunder et al. 2000a). In the inner plexiform layer, the pattern of GluR2 expression changes from a disoriented pattern at birth to a laminated pattern prior to eye-opening (Johansson et al. 2000), a manifestion of a reorganization of synaptic circuits.

Synaptogenesis in early development is activity-dependent (Wong and Ghosh 2002). The formation of synaptic contacts requires the mobility of dendritic structures regulated by spontaneous glutaminergic transmission. The rate of dendritic mobility was decreased by antagonists of both NMDA receptors and non-NMDA glutamate receptors (Wong et al. 2000). In the long term plasticity in the hippocampus and the visual cortex, NMDAR activation results in CaMKIla-mediated delivery of AMPAR to the synaptic membrane for enhanced synaptic efficacy, and CPEB1 mediates rapid local translation of CaMKIla mRNA in active synaptic sites. In the hippocampus, under a different stimulus - kainate induced seizure, CPEB3 regulates the translation of GluR2 mRNA (Huang et al. 2006). If similar machinery is harnessed for synaptogenesis, then one may relate such key molecules to CPEB. The expression pattern for CPEB3 presented here is similar to the pattern for retinal GluR2 (Dijk and Kamphuis 2004; Grunder et al. 2000a; Hack et al. 2001; Hamassaki-Britto et al. 1993; Kamphuis et al. 2003). Both are 
expressed in the same type of cells, and both are up-regulated in the first two weeks. Since CPEB3 protein becomes phosphorylated at Serine 197 in activated synaptosomes (Munton et al. 2007), a determination of the phosphorylation status of CPEB3 at each age would be useful to further establish the relationship of GluR2 and CPEB3. Manipulation of CPEB3 expression in vivo, including the use of knockout mice, could be used to advance the exploration of the role of CPEB3 in the retina. MAPK, BDNF/trkB, and certain cytoskeletal proteins are involved in synatogenesis as well (Job and Lagnado 1998; Schmitz et al. 1993; Oliveira et al. 2008; Cohen-Cory and Lom 2004; Liu et al. 2007a). MAPK and BDNF both demonstrated elongated poly $(A)$ tails during early development. Further experiments are needed to establish the relationship of their polyadenylation with particular CPEBs.

In addition to GluR2, several other synaptic molecules have been reported as being developmentally up-regulated in the retina, such as AMPAR subunits GluR1, GluR4, NMDAR subunits NR1, NR2A, NR2B, and CaMKIla (Grunder et al. 2000a; Chang and Chiao 2008; Xue and Cooper 2001; Xue et al. 2001). These molecules are expressed in a similar spatial pattern as CPEBs: in ganglion cells and amacrine cells. Additionally, our results on potential CPEB1 target mRNAs revealed that four out of eight genes were up-regulated during development: CaMKIla (which confirms the previous finding by Xue), Ppp2r2b, RBP-3, and Ttr. The patterns of these genes in early development were similar to that of CPEB1, 3 and 4 . It is yet to be determined whether any of the aforementioned mRNAs are suitable targets of CPEB3. Bioinformatics analysis screening for the U-rich hairpin loop structure in the 3' UTR of mRNAs, the binding site of CPEB3 protein (Huang et al. 2006) could be used to predict the suitability of the interaction between CPEB3 and particular mRNAs. In vivo binding assays could then be employed to further validate such mRNA-protein interactions (Huang et al. 2006). 


\section{CPEB1, CPEB3, CPEB4 and synaptic maturation}

Although most morphological and neurochemical properties of the retina (i.e., the numbers of conventional and ribbon synapses, the expression of receptors for neurotransmitters) have reached the adult level at the time of eye opening (Fisher 1979a; Sassoe-Pognetto and Wassle 1997), the maturation of synaptic function continues for several weeks after eye-opening. For example, the synaptic strength of the RGCs, measured as the frequency of spontaneous synaptic activity, was low around the time of eye opening, and surges around P25 both in spontaneous excitatory postsynaptic currents (sEPSCs) mediated by glutamate receptors and in spontaneous inhibitory postsynaptic currents (sIPSCs) mediated by GABA/glycine receptors (Tian and Copenhagen 2001). The establishment of ON versus OFF pathways and the dendritic pruning of the IPL occur to a large extent after eye opening.

The dendrites of RGCs ramify throughout the INL at the early postnatal time (Diao et al. 2004). Over development most of those dendrites achieve stratified patterns either in sublamina $a$ or sublamina $b$ of the INL. A significant portion of this morphological change occurs after eye opening. The ratio of bi-stratified RGCs (respond to both ON and OFF stimulus) to monostratified RGCs (respond to either ON or OFF stimulus) declined significantly in two to three weeks after eye opening (Tian and Copenhagen 2003).

The dendritical pruning in the inner plexiform layer is concurrent with the maturational alteration of bistratified ON-OFF responsive bipolar cells to monostratified ON or OFF responsive cells. It is postulated that the RGCs achieve their assignment to ON or OFF pathways by pruning their dendrites - removing "misplaced" dendrites and limiting their 
dendrites only in sublamina a or sublamina b. Both the alteration of ON and OFF pathways in RGCs and the dendritic pruning in the IPL are activity dependent. The agedependent decline of bistratified RGCs was completely blocked in dark-reared animals. Light deprivation also retarded the maturational loss of ON-OFF responsive RGCs (Tian and Copenhagen 2003). Similar retardation in the synaptic pruning of bistratified RGCs was also observed in pharmacological studies which mimic the effect of light deprivation. Injection of 2-amino-4-phosphonobutyrate (APB), a compound that specifically blocks the release of glutamate by cone-driven ON bipolar cells and rod driven bipolar cells, diminished the maturational stratification process of RGC dendrites (Bisti et al. 1998; Bodnarenko and Chalupa 1993; Bodnarenko et al. 1995). Such delays in alteration of ON and OFF pathways and dendritic refinement elicited by light deprivation were reversible both in young and adult animals, however, the time course of such recovery is age-dependent, with younger animals requiring longer time for recovery (Tian and Copenhagen 2001).

The mechanism underlying the activity-dependent maturation of ON and OFF pathways is yet to be fully delineated. One attractive hypothesis is that the retrainment or removal of a dendrite is regulated by NMDA receptors. Activity strengthens synaptic contacts that fire synchronously and eliminates the ones firing asynchronously. The developmental shift of predominant NR1/NR2B receptors to more NR1/NR2A receptors may be a key player in this mechanism. The downstream cascade of NMDAR activation is yet to be clarified for this process. One possible direction is to investigate whether CPEBs play a role following NMDAR activation. The expression of NR2A subunit is agedependent and is decreased in dark-reared developing retina (Xue and Cooper 2001). Our study demonstrated that CPEB1, CPEB3 and CPEB4 have a similar age-dependent up-regulation in the retina. Whether the up-regulation/activation of CPEBs is dependent 
on NMDAR activity could be determined in the future with the aid of the visual deprivation model. Agonists or antagonists of the glutamate receptors can also be applied in vivo. Synaptic enrichments of potential targets of CPEBs, such as CaMKII for CPEB1 and GluR2 for CPEB3, can also be studied in this scenario. The causal relationship is still in question: Is CPEB up-regulation driven by the process of synaptic maturation or vice versa?

6. Synaptic plasticity in the adult retina

Both our real-time PCR data and Western blot data indicated that CPEB1 and CPEB3, and CPEB4 continue to increase after synaptogenesis and synaptic maturation, and reaches a plateau in adulthood (P60). This suggested CPEBs are functional in the adult retina. This may be related to certain forms of synaptic plasticity in the adult life.

Neuronal plasticity has been historically characterized as a feature of the developing central nervous system, but there is an increasing amount of evidence which indicates that activity-dependent synaptic plasticity can occur both during development and adulthood. One form of activity dependent plasticity in the adult retina is regulated in the course of normal diurnal light/dark cycle. GluR2-containing AMPAR undergoes rapid cycling in the retinal ganglion cells and amacrine cells via endocytosis and exocytosis (Xia et al. 2006). Synaptic quiescence in darkness drives rapid cycling which leads to AMPARs altering from GluR2-containing to GluR2-lacking on synaptic membrane. On the contrary, synaptic activity in the light inhibits cycling and stabilizes AMPAR as predominantly GluR2-containing on the surface. The activation of AMPARs is critical for the switching between cycling and non-cycling states (Xia et al. 2007). The relationship between AMPAR cycling and synaptic function is not well understood, but is believed to 
have a role in certain forms of synaptic plasticity. Such cycling of AMPAR is present in hippocampus neurons as well, and is regulated by NMDAR activation and calcium influx (Biou et al. 2008). The cycling of GluR2-containing AMPARs plays a critical role in synaptic plasticity in the adult hippocampus (Luscher et al. 1999).

The expression of BDNF in the retina undergoes diurnal cycles under normal light/dark conditions. The level of BDNF is higher during the light than during the dark (Pollock et al. 2001, Cooper et al. 2008). BDNF/TrkB pathway has been proposed as a mediator in activity-dependent plasticity. BDNF can be retrogradely transported and act as a regulator of the bi-directional synaptic communication (An et al. 2008; Bramham and Messaoudi 2005; Carvalho et al. 2008; Cohen-Cory and Lom 2004; Tongiorgi et al. 1996; Tongiorgi et al. 2006). Under diurnal conditions BDNF may modulate the efficacy and stability of synaptic connections in visual circuits. Since BDNF has CPE and PAS motifs in its 3' UTR, the role of CPEBs in the regulation of BDNF during the light/dark cycles can be further explored using the diurnal model.

Increasing evidence demonstrates that the outer plexiform layer of adult retina also possesses some degree of synaptic plasticity. For example, ectopic dendrites grow to make new connections in the outer plexiform layer in diseased or ageing retina (Eliasieh et al. 2007; Sullivan et al. 2007). Such newly formed synapses are functional and capable of releasing neurotransmitters. Whether CPEB3, which demonstrated strong labeling in the OPL of the adult retina, is involved in such remodeling is a topic worth pursuing in the future.

It is conceivable that some "consensus" molecules may be involved in synaptic plasticity in both development and adulthood. Whether synaptic molecules such as GluR2 and CaMKIla work together with CPEBs in synaptic plasticity in the adult retina is a question 
also worth pursuing. It is likely that different members of the CPEB family, or even different isoforms of the same paralog, are involved in distinct forms of synaptic plasticity or in different stages of development.

7. Multiple CPEB3 isoforms in the retina suggest a level of complexity for CPEB3 activity

1) Multiple CPEB3 protein isoforms are present in the retina.

A previous study from Kandel's group identified two alternative splicing regions of CPEB3 (Theis et al. 2003), which accordingly give rise to four different isoforms of protein (isoform 1-4 in figure 29). In the current study, we identified a total of eight variants of CPEB3 transcripts and seven isoforms of CPEB3 protein in the database (figure 28, 29). Additionally, we reported for the first time a novel isoform of CPEB3 (figure 30c, "exon 11 missing").

Data derived from CPEB3 Western blots in the current study demonstrated a band as $\sim 75 \mathrm{kD}$ (figure $35 \mathrm{c}$ ) in the retina. This band may represent one or more of CPEB3 isoform $1-4$, of which the predicted size is $78 \mathrm{kD}, 77 \mathrm{kD}, 75.5 \mathrm{kD}$ and $74.5 \mathrm{kD}$, respectively (figure 29). The composition of the $\sim 75 \mathrm{kD}$ band could be further analyzed in future studies with techniques such as mass spectrometry (MS).

Results from CPEB3 Western blots demonstrated that besides the $\sim 75 \mathrm{kD}$ band, there were two prominent bands around $130 \mathrm{kD}$, one of which was absent when the antibody was pre-absorbed with CPEB3-recombinant protein. Of interest, the intensity of this band decreased during development of the retina. The $\sim 130 \mathrm{kD}$ band may be a dimer, a pre-protein, or a prion form. Each theory would explain the decrease in the relative 
amount of the $\sim 130 \mathrm{kD}$ band and the corresponding increase of the $75 \mathrm{kD}$ band. However, this band was not weakened under reducing and denaturing conditions (adding DTT or $\beta-M E$ and the samples boiled, data not shown), where S-S disulphate bands, if any, should be opened up. Therefore, it seems unlikely for this band to be a dimer of CPEB3. The speculation that it is a pre-CPEB3 needs further evidence. It could be investigated with the aid of Northern blots to determine whether a larger mRNA corresponding to this protein is present. It can also be addressed with the aid of immunoprecipitation and MS if sufficient amount of protein can be obtained. Another speculation is that $\sim 130 \mathrm{kD}$ is a prion form of CPEB3. Mouse CPEB3 shares unique characteristics with its Aplysia homolog (ApCPEB): a poly-glutamine track at the $\mathrm{N}$ terminus (figure 27). This short motif confers on several proteins, including the ApCPEB, the structure flexibility to form prion (Darnell 2003; Si et al. 2003b; Olson 2005). Further study with the aid of mutations or chimera proteins could be used to address whether the $\sim 130 \mathrm{kD}$ band is the prion form of CPEB3 in the mouse retina.

Two bands smaller than $75 \mathrm{kD}$ (figure $35 \mathrm{c}, 67 \mathrm{kD}$ and $62 \mathrm{kD}$, respectively) in our Western blots also disappeared when the antibody was pre-absorbed with the recombinant CPEB3. These two bands could be possible cleavage products from the $\sim 75 \mathrm{kD}$ band or the $130 \mathrm{kD}$ band. It is also possible that they are two different isoforms of CPEB3, since their sizes are consistent with that of isoform 7 and isoform 5, respectively (figure 29). Likewise their identities can be determined with mass spectrometry if sufficient material can be isolated from the retina.

Quantification of Western blots on CPEB3 demonstrated that the level of CPEB3 protein (the $75 \mathrm{kD}$ band) was significant up-regulated from age $P 1$ and $P 7$ to $P 12$. This is consistent with the change of CPEB3 mRNA levels during development. It is also in accordance with in situ hybridization results at different ages (data not shown). 
Synaptogenesis in the mouse retina occurs prominently before eye-opening. This prompted a likely correlation between the function of CPEB3 and synaptogenesis in the retina. It would be very interesting to look into the substrate mRNAs that are regulated by CPEB3 with regard to whether they are components for the construction of synapses. One of the factors now known to be regulated by CPEB3 in is GluR2 (Huang et al. 2006), which is implicated in basic synaptic transmission in the hippocampus (Meng et al. 2003).

2) CPEB3 variants provide for a higher level of regulation complexity than has been hitherto recognized.

The large number of variants in the coding region, together with variability in the 5' UTR, and 3' UTRs of CPEB3 provides evidence for an unexpected level of complexity in regulatory and functional properties of CPEB3. First of all, the differences in its protein sequences (resulting from alternative splicing in coding exons) may involve the attachment of discrete "tags" to protein isoforms so that they may function in different temporal and spatial patterns, possibly on different RNA targets, with different protein partners, or upon different stimuli. Activation, inhibition or even turnover could also be affected by the affinity for different kinases, phosphatases, endopeptidases. Secondly, the regulatory and functional properties can result from the differences in the 5' UTR and 3' UTR regions of the CPEB3 mRNAs. The UTRs may harbor signal motifs for localizing mRNAs to different sub-cellular compartments, subjecting the mRNA to distinct translation mechanisms, or degrading the mRNA via discrete pathways. One striking phenomenon is the presence of CPE motifs in the 3' UTR of CPEB3 mRNA, which casts CPEB3 mRNA as a good candidate target for CPEB1 protein. 
8. Comparison of CPEB1 and CPEB3:

1) CPEB1 and CPEB3 have different distribution patterns in the retina

Our study in the retina revealed different patterns of CPEB1 and CPEB3. First, in the RGC layers, CPEB3 is strongly expressed in the majority of the cell soma; while CPEB1 in expressed in a few cell soma. Secondly, both are strongly expressed in the INL. But CPEB1 demonstrated laminar structures. At least two layers are distinguishable in the IPL. In comparison, the pattern of CPEB3 in the IPL is more diffuse. Last, labeling in the OPL was strong for CPEB3, but barely detectable for CPEB1.

2) CPEB1 and CPEB3 may play different roles in the retina

The comparision between the expression patterns of CPEB1 and CPEB3 suggested that CPEB1 and CPEB3 may have different roles in the retina. Different distribution for CPEB1 and CPEB3 has been shown in the hippocampus, where CPEB3, but not CPEB1 was responsive to kainate acid induced seizure (Theis et al. 2003). Protein-RNA binding assays revealed that CPEB1 and CPEB3 recognize different subset of mRNA targets (Huang et al. 2006). Although postnatal development seemed to regulate the expression of both, CPEB1 and CPEB3 may respond to different types of stimuli in the retina. Other experimental paradigms such as light/ dark cycles, dark-rearing, or injury/ aging models may be used to further differentiate the two. 
3) There may exist a feedback loop between CPEB1 and CPEB3

One intriguing property of CPEB3 is that its MRNA contains two CPE motifs in the $3^{\prime}$ UTR. This casts CPEB3 as a good candidate for CPEB1-regulated translational control. Should this be indeed the case, CPEB3 may provide a feedback loop for CPEB1 activation. It could be a positive feedback which augments and expedites the effect of CPEB1 activation; or a negative feedback that eases the effect of CPEB1 activation.

In summary, our data described the expression patterns of four CPEBs in the retina. There were some similarities and discrepancies between the four, both spatially and temporally. The function of each CPEB in the retina is yet to be delineated. It is possible that some degree of functional compensation of redundancy exists between certain CPEBs. Based on the prevalent studies on CPEB1, which have been devoted to the region of hippocampus in the CNS, we speculate that CPEB1 and the other CPEB paralogs are involved in synaptic activity, possibly synaptogenesis and certain forms of synaptic plasticity in the retina. A variety of congenital or acquired conditions are the leading causes of retinal degeneration that leads to blindness. Current knowledge about the capacity for remodeling and restoration of the retina is of key significance for developing cures for degenerative retinal diseases. The exploration of the roles of CPEBs and their relative abilities to regulate protein translation in the retina will advance our understanding on the structure and function of the retina, especially pertaining to neural plasticity. 


\title{
CHAPTER VI \\ FUTURE DIRECTIONS
}

\begin{abstract}
Although some functional studies have been pursued for CPEB1, and to a less extent, for CPEB3, in the hippocampus and visual cortex, little is known with regard to the function of each CPEB in the retina. Some aspects of the function may be investigated with recapitulation of available techniques used in the other systems, in some current established in vitro or in vivo models.
\end{abstract}

1. In vitro studies: Identification of mRNA targets for each CPEB.

One of the important aspects regarding the functions of CPEB1-4 is the substrate specificity of each: the mRNA targets they recognize and mediate. Our study identified some potential mRNA targets for CPEB1. Additionally, potential targets of CPEB2-4 may be identified more extensively using the following approach 1). The interaction between the mRNA targets and individual CPEB proteins can be further validated using the following experiments 2) and 3). 
1) SELEX screening in combination with bioinformatic tools

Huang and colleagues used SELEX (systematic evolution of ligands by exponential enrichment) method to determine that the recognition of CPEB3 of its target mRNAs requires a secondary structure in the 3' UTR of the mRNA (Huang et al. 2006). A pool of random 25-mer RNAs was generated from an oligonucleotide library composed of random 25-mers flanked by constant regions for PCR. The RBD (RNA binding domain) of CPEB3 or CPEB4 were mixed with the mixed RNA pool. However, in the experiment followed, they found that the $3^{\prime}$ flanking sequence is critical for the formation of the secondary structure during the recognition of CPEB3. Since the 3 ' flanking sequence used in their experiment was a constant sequence which was arbitrary by nature, the sequence of the recognition motif derived from their experiment may be limited.

A modified version of this experiment can be performed with the following modification: Generate a pool of random 50-mer RNAs from an oligonucleotide library composed of random 50-mers flanked by constant regions for PCR. Use the pool of 50-mer RNA in the binding assays. (" 50 " is derived from: the length of the previous 25 -mer plus the length of the 3' flanking sequence). Follow the same procedures for the rest of the experiment.

This modification of the SELEX experiment is more inclusive and is likely to generate more variants of the recognition motif for the CPEB tested. Then extend from "CPE Detector" program, these variants can be screened in 3' UTR databases, and potential mRNA targets can be extracted computationally from the database. Promising mRNA candidates can be validated experimentally using the following in vitro binding assays or in cell knockdown/over-expression analysis. 
2) In vitro binding assays

Partial mRNAs of interest (with the 3' UTR) can be in vitro transcribed from PCR products using T7 promoters and radioactively labeled. Protein of interest (CPEB1-4) can be expressed as recombinant protein and then refolded to natural conformation if necessary, or purified from tissue as biologically active form using immunoprecipitation. The enriched RNA probes can then be incubated with specific protein. The RNA:protein complex can then be subjected to gel shift assay using PAGE gel. The presence or absence of binding can be verified by the shift or no-shift of the band from the free probe band. Synthesized mRNA fragments without binding sites for CPEBs can be used as a negative control to account for non-specific binding.

The in vitro binding assay can also be used in large scale screening from a biological sample. For example, total mRNA from the retina can be applied in incubation with CPEB proteins. The binding complex will then be washed, and the mRNA targets eluted and extracted. The identity of the mRNAs can be identified using microarray.

3) In cell knockdown/over-expression analysis

Cell knockdown or over-expression assay is another alternative to identify mRNAs associated with the CPEB. CPEB1 acts as a translational suppressor when inactivated, and an activator when activated, and the binding of CPEB1 to the mRNA is constitutive. This is also true for CPEB3 with respect to its interaction with GluR2. Therefore, a change in the translational levels of the target mRNAs should be detectable regardless of the stimulus (down-regulation without stimulus, up-regulation with appropriate stimulus). 
For potential candidates, for example, from SELEX screening in combination with bioinformatic tools, candidate proteins can be validated using quantitative assays such as Western blot. Expected alterations in the level of proteins would be a strong indicator of the association of the mRNA with CPEB. However, verification of the physical binding of the two is still desirable.

The total protein extraction from CPEB knockdown/over-expression samples can also be used in high-throughput analysis such as proteomics. The identity of down-regulated proteins (in the absence of stimulus) or up-regulated proteins (in the presence of an appropriate stimulus) can be verified with the protein extraction from naïve cells as a control.

\section{In vivo studies}

In vivo studies should be the eventual goal to delineate the role of CPEB in the retina. Since it is highly likely that different CPEBs recognize discrete mRNA substrates and may be responsive to distinct stimuli, it is critical that appropriate models are used. Some experimental paradigms can be tested as discussed below:

\section{1) Diurnal cycle model}

As described in previous literature, GluR2-containing AMPAR undergoes rapid cycling in ON ganglion cells under normal light/dark cycles (Xia et al. 2007; Xia et al. 2006). The rapid cycling of AMPARs is believed to maintain synaptic transmission and may be related to several forms of synaptic plasticity. Light adaptation stabilizes it in a nontrafficking state, while dark adaptation switches it into a state of rapid cycling. The 
activation of AMPAR is critical for the switch (Xia et al. 2006), however the underlying mechanism is yet to be discovered.

GluR2 is believed to be a target of CPEB3 in the hippocampus (Huang et al 2006). However, whether CPEB3 mediates the translation of GluR2, or indirectly leads to the activation of AMPAR in the retina, is a question yet to be addressed. The diurnal model may serve as one diagram to explore this issue. siRNA of CPEB3 can be delivered to retinal ganglion cells via intravitreal injection. The expression of GluR2 or the cycling pattern of GluR2-containing AMPAR can then be determined.

2) Developmental model

The inner retina undergoes profound maturational remodeling during post-natal development. Such process has been proven to be activity-dependent. Our study demonstrated that CPEB1, 3 and 4 were up-regulated during development. CPEB1 and CPEB3 demonstrated a different spatial pattern. It is not known whether the expression of one or more CPEBs is activity dependent. Dark-rearing can be used during development to investigate this question. Both the spatial localization of the CPEBs and the quantitative level of CPEBs should be observed in dark-reared animals and in normal-reared control.

3) Knockout mouse model

CPEB1 knockout mice may be used in comparison to normal littermates for the polyadenylation and translation of target mRNAs. Similarly, CPEB3 knockout mice can be generated; the translation of known or potential mRNA targets of CPEB3 can be 
evaluated. Immunohistochemistry can be used to determine affected areas/cell types of the retina. Electrophysiology properties such as ERG, OPs, and EPSPs can be measured to infer the effects of CPEB knockout on the functioning of the retina. Behavior studies can be designed, if plausible, to elaluate the overall performance of vision.

4) Photoreceptor degeneration models

The outer plexiform layer of the retina manifested a certain degree of plasticity in mutant, diseases or aging that cause photoreceptor degeneration. Following the loss of synaptic contacts in the outer plexiform layer, horizontal cells and bipolar cells in the inner nuclear cells outgrow dendrites to the outer nuclear layer to form new connections with photoreceptors. CPEB3 appeared to be present at the outer plexiform layer in the adult mice (P60). The localization of CPEB3 with respect to pre-synaptic or post-synaptic compartment needs to be further addressed. The expression pattern of CPEB3 can be explored in the above models, which may provide some insights for its function in the outer plexiform layer.

5) Application of kinase inhibitors, neurotransmitter agonists or antagonists

Four CPEBs may be responsive to different stimuli. For example, CPEB3 and CPEB4, but not CPEB1, were up-regulated in kainate induced seizure in the hippocampus (Huang et al. 2006). With the appropriate stimulus, the cascade of the activation of each CPEB can be further delineated. CPEB1 is known to be activated by aurora kinase or CaMKII, both of which require the activation of NMDA receptors. Sequence analysis 
indicated that CPEB2-4 do not possess phosphorylation site for aurora kinase, but appear to be substrates for PKA, CaMKII and p70S6 kinase. Inhibitors of these kinases can be used to verify the cascade of phosphorylation under appropriate stimulus. Agonists or antagonists of different types of neurotransmitter receptors can also be applied to infer the requirement for the activation of receptors for each CPEB. 


\section{REFERENCES}

Aakalu G, Smith WB, Nguyen N, Jiang C, Schuman EM 2001. Dynamic visualization of local protein synthesis in hippocampal neurons. Neuron 30:489-502.

Aartsen WM, Kantardzhieva A, Klooster J, van Rossum AG, van de Pavert SA, Versteeg I, Cardozo BN, Tonagel F, Beck SC, Tanimoto N, Seeliger MW, Wijnholds J 2006. Mpp4 recruits Psd95 and Veli3 towards the photoreceptor synapse. Hum Mol Genet 15:12911302.

Alarcon JM, Hodgman R, Theis M, Huang YS, Kandel ER, Richter JD 2004. Selective modulation of some forms of schaffer collateral-CA1 synaptic plasticity in mice with a disruption of the CPEB-1 gene. Learn Mem 11:318-327.

Ambrosini A, Bresciani L, Fracchia S, Brunello N, Racagni G 1995. Metabotropic glutamate receptors negatively coupled to adenylate cyclase inhibit N-methyl-Daspartate receptor activity and prevent neurotoxicity in mesencephalic neurons in vitro. Mol Pharmacol 47:1057-1064.

An JJ, Gharami K, Liao GY, Woo NH, Lau AG, Vanevski F, Torre ER, Jones KR, Feng Y, Lu B, Xu B 2008. Distinct role of long 3' UTR BDNF mRNA in spine morphology and synaptic plasticity in hippocampal neurons. Cell 134:175-187.

Araki CM, Hamassaki-Britto DE 2000. Calretinin co-localizes with the NMDA receptor subunit NR1 in cholinergic amacrine cells of the rat retina. Brain Res 869:220-224.

Atkins CM, Selcher JC, Petraitis JJ, Trzaskos JM, Sweatt JD 1998. The MAPK cascade is required for mammalian associative learning. Nat Neurosci 1:602-609.

Awobuluyi M, Yang J, Ye Y, Chatterton JE, Godzik A, Lipton SA, Zhang D 2007. Subunit-specific roles of glycine-binding domains in activation of NR1/NR3 N-methyl-Daspartate receptors. Mol Pharmacol 71:112-122.

Baba A, Yasui T, Fujisawa S, Yamada RX, Yamada MK, Nishiyama N, Matsuki N, Ikegaya $Y$ 2003. Activity-evoked capacitative Ca2+ entry: implications in synaptic plasticity. J Neurosci 23:7737-7741.

Bading $\mathrm{H} 2000$. Transcription-dependent neuronal plasticity the nuclear calcium hypothesis. Eur J Biochem 267:5280-5283.

Barria A, Muller D, Derkach V, Griffith LC, Soderling TR 1997. Regulatory phosphorylation of AMPA-type glutamate receptors by CaM-KII during long-term potentiation. Science 276:2042-2045. 
Bally-Cuif L, Schatz WJ, Ho RK 1998. Characterization of the zebrafish Orb/CPEBrelated RNA binding protein and localization of maternal components in the zebrafish oocyte. Mech Dev 77:31-47.

Beresewicz M 2007. [Scaffold proteins (MAGUK, Shank and Homer) in postsynaptic density in the central nervous system]. Postepy Biochem 53:188-197.

Barnard DC, Ryan K, Manley JL, Richter JD 2004. Symplekin and XGLD-2 are required for CPEB-mediated cytoplasmic polyadenylation. Cell 119:641-651.

Binder DK, Scharfman HE 2004. Brain-derived neurotrophic factor. Growth Factors 22:123-131.

Biou V, Bhattacharyya S, Malenka RC 2008. Endocytosis and recycling of AMPA receptors lacking GluR2/3. Proc Natl Acad Sci USA 105:1038-1043.

Bisti S, Gargini C, Chalupa LM 1998. Blockade of glutamate-mediated activity in the developing retina perturbs the functional segregation of ON and OFF pathways. $J$ Neurosci 18:5019-5025.

Blanks JC, Bok D 1977. An autoradiographic analysis of postnatal cell proliferation in the normal and degenerative mouse retina. J Comp Neurol 174:317-327.

Blum S, Moore AN, Adams F, Dash PK 1999. A mitogen-activated protein kinase cascade in the CA1/CA2 subfield of the dorsal hippocampus is essential for long-term spatial memory. J Neurosci 19:3535-3544.

Bodnarenko SR, Chalupa LM 1993. Stratification of ON and OFF ganglion cell dendrites depends on glutamate-mediated afferent activity in the developing retina. Nature 364:144-146.

Bodnarenko SR, Jeyarasasingam G, Chalupa LM 1995. Development and regulation of dendritic stratification in retinal ganglion cells by glutamate-mediated afferent activity. $\mathrm{J}$ Neurosci 15:7037-7045.

Boeckers TM 2006. The postsynaptic density. Cell Tissue Res 326:409-422.

Bortolotto ZA, Nistico R, More JC, Jane DE, Collingridge GL 2005. Kainate receptors and mossy fiber LTP. Neurotoxicology 26:769-777.

Bramham CR, Messaoudi E 2005. BDNF function in adult synaptic plasticity: the synaptic consolidation hypothesis. Prog Neurobiol 76:99-125.

Bramham CR 2008. Local protein synthesis, actin dynamics, and LTP consolidation. Curr Opin Neurobiol 18:524-531.

Braun AP, Schulman H 1995. The multifunctional calcium/calmodulin-dependent protein kinase: from form to function. Annu Rev Physiol 57:417-445. 
Brocke L, Srinivasan M, Schulman H 1995. Developmental and regional expression of multifunctional $\mathrm{Ca2}+/$ calmodulin-dependent protein kinase isoforms in rat brain. $\mathrm{J}$ Neurosci 15:6797-6808.

Buisson A, Yu SP, Choi DW 1996. DCG-IV selectively attenuates rapidly triggered NMDA-induced neurotoxicity in cortical neurons. Eur J Neurosci 8:138-143.

Burgin KE, Waxham MN, Rickling S, Westgate SA, Mobley WC, Kelly PT 1990. In situ hybridization histochemistry of $\mathrm{Ca} 2+/$ calmodulin-dependent protein kinase in developing rat brain. J Neurosci 10:1788-1798.

Cajal SR 1893. La retine des vertebres. La Cellule 9:17-257.

Cajal SR 1892. The Structure of the Retina. (Translated by S.A. Thorpe and M. Glickstein), Springfield, II., Thomas, 1972.

Carvalho AL, Caldeira MV, Santos SD, Duarte CB 2008. Role of the brain-derived neurotrophic factor at glutamatergic synapses. Br J Pharmacol 153 Suppl 1:S310-S324.

Casini G, Rickman DW, Brecha NC 1995. All amacrine cell population in the rabbit retina: identification by parvalbumin immunoreactivity. J Comp Neurol 356:132-142.

Catania MV, D'Antoni S, Bonaccorso CM, Aronica E, Bear MF, Nicoletti F 2007. Group I metabotropic glutamate receptors: a role in neurodevelopmental disorders? Mol Neurobiol 35:298-307.

Chang B, Heckenlively JR, Bayley PR, Brecha NC, Davisson MT, Hawes NL, Hirano AA, Hurd RE, Ikeda A, Johnson BA, McCall MA, Morgans CW, Nusinowitz S, Peachey NS, Rice DS, Vessey KA, Gregg RG 2006. The nob2 mouse, a null mutation in Cacna1f: anatomical and functional abnormalities in the outer retina and their consequences on ganglion cell visual responses. Vis Neurosci 23:11-24.

Chang JS, Tan L, Schedl P 1999. The Drosophila CPEB homolog, orb, is required for oskar protein expression in oocytes. Dev Biol 215:91-106.

Chang YC, Chiao CC 2008. Localization and functional mapping of AMPA receptor subunits in the developing rabbit retina. Invest Ophthalmol Vis Sci 49:5619-5628.

Chapman PF, Frenguelli BG, Smith A, Chen CM, Silva AJ 1995. The alpha$\mathrm{Ca} 2+/$ calmodulin kinase II: a bidirectional modulator of presynaptic plasticity. Neuron 14:591-597.

Chatterton JE, Awobuluyi M, Premkumar LS, Takahashi H, Talantova M, Shin Y, Cui J, Tu S, Sevarino KA, Nakanishi N, Tong G, Lipton SA, Zhang D 2002. Excitatory glycine receptors containing the NR3 family of NMDA receptor subunits. Nature 415:793-798.

Cheetham CE, Hammond MS, McFarlane R, Finnerty GT 2008. Altered sensory experience induces targeted rewiring of local excitatory connections in mature neocortex. J Neurosci 28:9249-9260. 
Cheung HK, Serano TL, Cohen RS 1992. Evidence for a highly selective RNA transport system and its role in establishing the dorsoventral axis of the Drosophila egg.

Development 114:653-661.

Christerson LB, McKearin DM 1994. orb is required for anteroposterior and dorsoventral patterning during Drosophila oogenesis. Genes Dev 8:614-628.

Christie BR, Magee JC, Johnston D 1996. The role of dendritic action potentials and $\mathrm{Ca} 2+$ influx in the induction of homosynaptic long-term depression in hippocampal CA1 pyramidal neurons. Learn Mem 3:160-169.

Chu Z, Hablitz JJ 2000. Quisqualate induces an inward current via mGluR activation in neocortical pyramidal neurons. Brain Res 879:88-92.

Claes E, Seeliger M, Michalakis S, Biel M, Humphries P, Haverkamp S 2004. Morphological characterization of the retina of the CNGA3(-/-)Rho(-/-) mutant mouse lacking functional cones and rods. Invest Ophthalmol Vis Sci 45:2039-2048.

Clegg KB, Piko L 1982. RNA synthesis and cytoplasmic polyadenylation in the one-cell mouse embryo. Nature 295:343-344.

Clegg KB, Piko L 1983a. Poly(A) length, cytoplasmic adenylation and synthesis of poly $(A)+$ RNA in early mouse embryos. Dev Biol 95:331-341.

Clegg KB, Piko L 1983b. Quantitative aspects of RNA synthesis and polyadenylation in 1-cell and 2-cell mouse embryos. J Embryol Exp Morphol 74:169-182.

Cohen-Cory S, Lom B 2004. Neurotrophic regulation of retinal ganglion cell synaptic connectivity: from axons and dendrites to synapses. Int J Dev Biol 48:947-956.

Cooper NG, Laabich A, Fan W, Wang X 2008. The relationship between neurotrophic factors and CaMKII in the death and survival of retinal ganglion cells. Prog Brain Res 173:521-540.

Cooper NG, Wei X, Liu N 1995. Onset of expression of the alpha subunit of $\mathrm{Ca2+/calmodulin-dependent} \mathrm{protein} \mathrm{kinase} \mathrm{II}$ and a novel related protein in the developing retina. J Mol Neurosci 6:75-89.

Crooks J, Kolb H 1992. Localization of GABA, glycine, glutamate and tyrosine hydroxylase in the human retina. J Comp Neurol 315:287-302.

Cummings JA, Mulkey RM, Nicoll RA, Malenka RC 1996. Ca2+ signaling requirements for long-term depression in the hippocampus. Neuron 16:825-833.

Darnell RB 2003. Memory, synaptic translation, and...prions? Cell 115:767-768.

Diao L, Sun W, Deng Q, He S 2004. Development of the mouse retina: emerging morphological diversity of the ganglion cells. J Neurobiol 61:236-249. 
Dick O, Tom DS, Altrock WD, Ammermuller J, Weiler R, Garner CC, Gundelfinger ED, Brandstatter $\mathrm{JH}$ 2003. The presynaptic active zone protein bassoon is essential for photoreceptor ribbon synapse formation in the retina. Neuron 37:775-786.

Dijk F, Kamphuis W 2004. Ischemia-induced alterations of AMPA-type glutamate receptor subunit. Expression patterns in the rat retina--an immunocytochemical study. Brain Res 997:207-221.

Dorland's Illustrated Medical Dictionary, 29 ${ }^{\text {th }}$ Edition. 2000. W.B. Saunders Company.

Dowling JE 1987. Wiring of the Retina. In: The retina: an approachable part of the brain. pp. 42-81. Cambridge, Mass. Belknap Press of Harvard University Press.

Dwork AJ, Cavallaro T, Martone RL, Goodman DS, Schon EA, Herbert J 1990. Distribution of transthyretin in the rat eye. Invest Ophthalmol Vis Sci 31:489-496.

Du L, Richter JD 2005. Activity-dependent polyadenylation in neurons. RNA 11:13401347.

Eckenstein F, Thoenen H 1982. Production of specific antisera and monoclonal antibodies to choline acetyltransferase: characterization and use for identification of cholinergic neurons. EMBO J 1:363-368.

Eliasieh K, Liets LC, Chalupa LM 2007. Cellular reorganization in the human retina during normal aging. Invest Ophthalmol Vis Sci 48:2824-2830.

Endoh T 2004. Characterization of modulatory effects of postsynaptic metabotropic glutamate receptors on calcium currents in rat nucleus tractus solitarius. Brain Res 1024:212-224.

English JD, Sweatt JD 1997. A requirement for the mitogen-activated protein kinase cascade in hippocampal long term potentiation. J Biol Chem 272:19103-19106.

English JD, Sweatt JD 1996. Activation of p42 mitogen-activated protein kinase in hippocampal long term potentiation. J Biol Chem 271:24329-24332.

Ephrussi A, Dickinson LK, Lehmann R 1991. Oskar organizes the germ plasm and directs localization of the posterior determinant nanos. Cell 66:37-50.

Famiglietti EV, Jr., Kolb H 1976. Structural basis for ON-and OFF-center responses in retinal ganglion cells. Science 194:193-195.

Ferrandon D, Elphick L, Nusslein-Volhard C, St JD 1994. Staufen protein associates with the 3'UTR of bicoid mRNA to form particles that move in a microtubule-dependent manner. Cell 79:1221-1232.

Fields RD, Eshete F, Dudek S, Ozsarac N, Stevens B 2001. Regulation of gene expression by action potentials: dependence on complexity in cellular information processing. Novartis Found Symp 239:160-172. 
Fisher LJ 1979a. Development of synaptic arrays in the inner plexiform layer of neonatal mouse retina. J Comp Neurol 187:359-372.

Fisher LJ 1979b. Development of retinal synaptic arrays in the inner plexiform layer of dark-reared mice. J Embryol Exp Morphol 54:219-227.

Fox CA, Sheets MD, Wickens MP 1989. Poly(A) addition during maturation of frog oocytes: distinct nuclear and cytoplasmic activities and regulation by the sequence UUUUUAU. Genes Dev 3:2151-2162.

Frey U, Muller M, Kuhl D 1996. A different form of long-lasting potentiation revealed in tissue plasminogen activator mutant mice. J Neurosci 16:2057-2063.

Funauchi M, Tsumoto T, Nishigori A, Yoshimura Y, Hidaka H 1992. Long-term depression is induced in $\mathrm{Ca} 2+/$ calmodulin kinase-inhibited visual cortex neurons. Neuroreport 3:173-176.

Gebauer F, Richter JD 1996. Mouse cytoplasmic polyadenylylation element binding protein: an evolutionarily conserved protein that interacts with the cytoplasmic polyadenylylation elements of c-mos mRNA. Proc Natl Acad Sci USA 93:14602-14607.

Genoux D, Montgomery JM 2007. Glutamate receptor plasticity at excitatory synapses in the brain. Clin Exp Pharmacol Physiol 34:1058-1063.

Gordon JA, Cioffi D, Silva AJ, Stryker MP 1996. Deficient plasticity in the primary visual cortex of alpha-calcium/calmodulin-dependent protein kinase II mutant mice. Neuron 17:491-499.

Groisman I, Huang YS, Mendez R, Cao Q, Theurkauf W, Richter JD 2000. CPEB, maskin, and cyclin B1 mRNA at the mitotic apparatus: implications for local translational control of cell division. Cell 103:435-447.

Groisman I, Jung MY, Sarkissian M, Cao Q, Richter JD 2002. Translational control of the embryonic cell cycle. Cell 109:473-483.

Greger IH, Ziff EB, Penn AC 2007. Molecular determinants of AMPA receptor subunit assembly. Trends Neurosci 30:407-416.

Grunder T, Kohler K, Guenther E 2000a. Distribution and developmental regulation of AMPA receptor subunit proteins in rat retina. Invest Ophthalmol Vis Sci 41:3600-3606.

Grunder T, Kohler K, Kaletta A, Guenther E 2000b. The distribution and developmental regulation of NMDA receptor subunit proteins in the outer and inner retina of the rat. $J$ Neurobiol 44:333-342.

Hack I, Frech M, Dick O, Peichl L, Brandstatter JH 2001. Heterogeneous distribution of AMPA glutamate receptor subunits at the photoreceptor synapses of rodent retina. Eur $\mathrm{J}$ Neurosci 13:15-24.

Haenlin M, Roos C, Cassab A, Mohier E 1987. Oocyte-specific transcription of fs(1)K10: a Drosophila gene affecting dorsal-ventral developmental polarity. EMBO J 6:801-807. 
Haeseleer F, Imanishi Y, Maeda T, Possin DE, Maeda A, Lee A, Rieke F, Palczewski K 2004. Essential role of Ca2+-binding protein 4 , a Cav1.4 channel regulator, in photoreceptor synaptic function. Nat Neurosci 7:1079-1087.

Hamassaki-Britto DE, Hermans-Borgmeyer I, Heinemann S, Hughes TE 1993. Expression of glutamate receptor genes in the mammalian retina: the localization of GluR1 through GluR7 mRNAs. J Neurosci 13:1888-1898.

Hanley JG 2008. PICK1: a multi-talented modulator of AMPA receptor trafficking. Pharmacol Ther 118:152-160.

Hayashi T, Umemori H, Mishina M, Yamamoto T 1999. The AMPA receptor interacts with and signals through the protein tyrosine kinase Lyn. Nature 397:72-76.

He X, Yang F, Xie Z, Lu B 2000. Intracellular $\mathrm{Ca}(2+)$ and $\mathrm{Ca}(2+) /$ calmodulin-dependent kinase II mediate acute potentiation of neurotransmitter release by neurotrophin-3. J Cell Biol 149:783-792.

Heine M, Groc L, Frischknecht R, Beique JC, Lounis B, Rumbaugh G, Huganir RL, Cognet L, Choquet D 2008. Surface mobility of postsynaptic AMPARs tunes synaptic transmission. Science 320:201-205.

Huang YS, Jung MY, Sarkissian M, Richter JD 2002. N-methyl-D-aspartate receptor signaling results in Aurora kinase-catalyzed CPEB phosphorylation and alpha CaMKII mRNA polyadenylation at synapses. EMBO J 21:2139-2148.

Huang YS, Kan MC, Lin CL, Richter JD 2006. CPEB3 and CPEB4 in neurons: analysis of RNA-binding specificity and translational control of AMPA receptor GluR2 mRNA. EMBO J 25:4865-4876.

Huarte J, Belin D, Vassalli A, Strickland S, Vassalli JD 1987. Meiotic maturation of mouse oocytes triggers the translation and polyadenylation of dormant tissue-type plasminogen activator mRNA. Genes Dev 1:1201-1211.

Huettner JE 2003. Kainate receptors and synaptic transmission. Prog Neurobiol 70:387407.

Hughes TE, Hermans-Borgmeyer I, Heinemann S 1992. Differential expression of glutamate receptor genes (GluR1-5) in the rat retina. Vis Neurosci 8:49-55.

Huntley GW, Gil O, Bozdagi O 2002. The cadherin family of cell adhesion molecules: multiple roles in synaptic plasticity. Neuroscientist 8:221-233.

Isaac JT, Ashby M, McBain CJ 2007. The role of the GluR2 subunit in AMPA receptor function and synaptic plasticity. Neuron 54:859-871.

Jansen HG, Hawkins RK, Sanyal S 1997. Synaptic growth in the rod terminals of mice after partial photoreceptor cell loss: a three-dimensional ultrastructural study. Microsc Res Tech 36:96-105. 
Jansen HG, Sanyal S 1992. Synaptic plasticity in the rod terminals after partial photoreceptor cell loss in the heterozygous rds mutant mouse. J Comp Neurol 316:117125.

Job C, Lagnado L 1998. Calcium and protein kinase C regulate the actin cytoskeleton in the synaptic terminal of retinal bipolar cells. J Cell Biol 143:1661-1672.

Johansson K, Bruun A, Torngren M, Ehinger B 2000. Development of glutamate receptor subunit 2 immunoreactivity in postnatal rat retina. Vis Neurosci 17:737-742.

Jorntell H, Hansel $\mathrm{C}$ 2006. Synaptic memories upside down: bidirectional plasticity at cerebellar parallel fiber-Purkinje cell synapses. Neuron 52:227-238.

Ju W, Morishita W, Tsui J, Gaietta G, Deerinck TJ, Adams SR, Garner CC, Tsien RY, Ellisman MH, Malenka RC 2004. Activity-dependent regulation of dendritic synthesis and trafficking of AMPA receptors. Nat Neurosci 7:244-253.

Kamiya H, Ozawa S, Manabe T 2002. Kainate receptor-dependent short-term plasticity of presynaptic $\mathrm{Ca} 2+$ influx at the hippocampal mossy fiber synapses. J Neurosci 22:9237-9243.

Kamphuis W, Klooster J, Dijk F 2003. Expression of AMPA-type glutamate receptor subunit (GluR2) in ON-bipolar neurons in the rat retina. J Comp Neurol 455:172-186.

Kandel ER, Schwartz JH, Jessell TM 1991. Principles of neural science, $3^{\text {th }}$ edition. Published by Elsevier.

Kandel ER, Schwartz JH, Jessell TM 2000. Principles of neural science, $4^{\text {th }}$ edition. Published by McGraw-Hill Professional.

Kang TH, Ryu YH, Kim IB, Oh GT, Chun MH 2004. Comparative study of cholinergic cells in retinas of various mouse strains. Cell Tissue Res 317:109-115.

Kano M, Hashimoto K, Tabata T 2008. Type-1 metabotropic glutamate receptor in cerebellar Purkinje cells: a key molecule responsible for long-term depression, endocannabinoid signalling and synapse elimination. Philos Trans R Soc Lond B Biol Sci 363:2173-2186.

Karmarkar UR, Dan Y 2006. Experience-dependent plasticity in adult visual cortex. Neuron 52:577-585.

Keady BT, Kuo P, Martinez SE, Yuan L, Hake LE 2007. MAPK interacts with XGef and is required for CPEB activation during meiosis in Xenopus oocytes. J Cell Sci 120:10931103.

Kenchappa RS, Zampieri N, Chao MV, Barker PA, Teng HK, Hempstead BL, Carter BD 2006. Ligand-dependent cleavage of the P75 neurotrophin receptor is necessary for NRIF nuclear translocation and apoptosis in sympathetic neurons. Neuron 50:219-232.

Kim CH, Lee J, Lee JY, Roche KW 2008. Metabotropic glutamate receptors: phosphorylation and receptor signaling. J Neurosci Res 86:1-10. 
Koh DS, Burnashev N, Jonas P 1995. Block of native Ca(2+)-permeable AMPA receptors in rat brain by intracellular polyamines generates double rectification. $J$ Physiol $486(2): 305-312$.

Kolb H 1997. Amacrine cells of the mammalian retina: neurocircuitry and functional roles. Eye $11(6): 904-923$

Kolb H, Linberg KA, Fisher SK 1992. Neurons of the human retina: a Golgi study. J Comp Neurol 318:147-187.

Kullmann DM, Lamsa K 2008. Roles of distinct glutamate receptors in induction of antiHebbian long-term potentiation. J Physiol 586:1481-1486.

Kurihara Y, Tokuriki M, Myojin R, Hori T, Kuroiwa A, Matsuda Y, Sakurai T, Kimura M, Hecht NB, Uesugi S 2003. CPEB2, a novel putative translational regulator in mouse haploid germ cells. Biol Reprod 69:261-268.

Laabich A, Li G, Cooper NG 2000. Calcium/calmodulin-dependent protein kinase II containing a nuclear localizing signal is altered in retinal neurons exposed to $\mathrm{N}$-methylD-aspartate. Brain Res Mol Brain Res 76:253-265.

Lantz V, Ambrosio L, Schedl P 1992. The Drosophila orb gene is predicted to encode sex-specific germline RNA-binding proteins and has localized transcripts in ovaries and early embryos. Development 115:75-88.

Lantz V, Chang JS, Horabin JI, Bopp D, Schedl P 1994. The Drosophila orb RNAbinding protein is required for the formation of the egg chamber and establishment of polarity. Genes Dev 8:598-613.

Lau CG, Zukin RS 2007. NMDA receptor trafficking in synaptic plasticity and neuropsychiatric disorders. Nat Rev Neurosci 8:413-426.

Lea PM, Custer SJ, Vicini S, Faden Al 2002. Neuronal and glial mGluR5 modulation prevents stretch-induced enhancement of NMDA receptor current. Pharmacol Biochem Behav 73:287-298.

Lee HK, Choi SS, Han EJ, Han KJ, Suh HW 2003. Role of glutamate receptors and an on-going protein synthesis in the regulation of phosphorylation of $\mathrm{Ca}+$ /calmodulindependent protein kinase II in the CA3 hippocampal region in mice administered with kainic acid intracerebroventricularly. Neurosci Lett 348:93-96.

Lehmann R, Nusslein-Volhard C 1991. The maternal gene nanos has a central role in posterior pattern formation of the Drosophila embryo. Development 112:679-691.

Leonard AS, Lim IA, Hemsworth DE, Horne MC, Hell JW 1999. Calcium/calmodulindependent protein kinase II is associated with the N-methyl-D-aspartate receptor. Proc Natl Acad Sci USA 96:3239-3244.

Liu LO, Li G, McCall MA, Cooper NG 2000. Photoreceptor regulated expression of $\mathrm{Ca}(2+) /$ calmodulin-dependent protein kinase II in the mouse retina. Brain Res Mol Brain Res 82:150-166. 
Liu N, Cooper NG 1996. The Ca2+/calmodulin-dependent protein kinase II-associated protein complex isolated from chicken retina. $J$ Mol Neurosci 7:1-12.

Liu X, Grishanin RN, Tolwani RJ, Renteria RC, Xu B, Reichardt LF, Copenhagen DR 2007a. Brain-derived neurotrophic factor and TrkB modulate visual experiencedependent refinement of neuronal pathways in retina. J Neurosci 27:7256-7267.

Liu XB, Murray KD, Jones EG 2004. Switching of NMDA receptor 2A and 2B subunits at thalamic and cortical synapses during early postnatal development. J Neurosci 24:88858895.

Liu Y, Wong TP, Aarts M, Rooyakkers A, Liu L, Lai TW, Wu DC, Lu J, Tymianski M, Craig AM, Wang YT 2007b. NMDA receptor subunits have differential roles in mediating excitotoxic neuronal death both in vitro and in vivo. J Neurosci 27:2846-2857.

Loeliger M, Rees S 2005. Immunocytochemical development of the guinea pig retina. Exp Eye Res 80:9-21.

Luitjens C, Gallegos M, Kraemer B, Kimble J, Wickens M 2000. CPEB proteins control two key steps in spermatogenesis in C. elegans. Genes Dev 14:2596-2609.

Lüscher C, Xia H, Beattie EC, Carroll RC, von ZM, Malenka RC, Nicoll RA 1999. Role of AMPA receptor cycling in synaptic transmission and plasticity. Neuron 24:649-658.

Lüscher C, Nicoll RA, Malenka RC, Muller D 2000. Synaptic plasticity and dynamic modulation of the postsynaptic membrane. Nat Neurosci 3:545-550.

MacDonald JF, Jackson MF, Beazely MA 2006. Hippocampal long-term synaptic plasticity and signal amplification of NMDA receptors. Crit Rev Neurobiol 18:71-84.

MacNeil MA, Masland RH 1998. Extreme diversity among amacrine cells: implications for function. Neuron 20:971-982.

Mansergh F, Orton NC, Vessey JP, Lalonde MR, Stell WK, Tremblay F, Barnes S, Rancourt DE, Bech-Hansen NT 2005. Mutation of the calcium channel gene Cacna1f disrupts calcium signaling, synaptic transmission and cellular organization in mouse retina. Hum Mol Genet 14:3035-3046.

Marquardt T, Gruss P 2002. Generating neuronal diversity in the retina: one for nearly all. Trends Neurosci 25:32-38.

Mayer ML 2005. Glutamate receptor ion channels. Curr Opin Neurobiol 15:282-288.

Mayer ML, MacDermott AB, Westbrook GL, Smith SJ, Barker JL 1987. Agonist- and voltage-gated calcium entry in cultured mouse spinal cord neurons under voltage clamp measured using arsenazo III. J Neurosci 7:3230-3244.

McGrew LL, Dworkin-Rastl E, Dworkin MB, Richter JD 1989. Poly(A) elongation during Xenopus oocyte maturation is required for translational recruitment and is mediated by a short sequence element. Genes Dev 3:803-815. 
McGrew LL, Richter JD 1990. Translational control by cytoplasmic polyadenylation during Xenopus oocyte maturation: characterization of cis and trans elements and regulation by cyclin/MPF. EMBO J 9:3743-3751.

Meldrum B, Garthwaite J 1990. Excitatory amino acid neurotoxicity and neurodegenerative disease. Trends Pharmacol Sci 11:379-387.

Mendez R, Murthy KG, Ryan K, Manley JL, Richter JD 2000. Phosphorylation of CPEB by Eg2 mediates the recruitment of CPSF into an active cytoplasmic polyadenylation complex. Mol Cell 6:1253-1259.

Meng $Y$, Zhang $Y$, Jia $Z$ 2003. Synaptic transmission and plasticity in the absence of AMPA glutamate receptor GluR2 and GluR3. Neuron 39:163-176.

Micklem DR, Adams J, Grunert S, St JD 2000. Distinct roles of two conserved Staufen domains in oskar mRNA localization and translation. EMBO J 19:1366-1377.

Mizui T, Takahashi $H$, Sekino $Y$, Shirao T 2005. Overexpression of drebrin A in immature neurons induces the accumulation of F-actin and PSD-95 into dendritic filopodia, and the formation of large abnormal protrusions. Mol Cell Neurosci 30:630-638.

Mojumder DK, Wensel TG, Frishman LJ 2008. Subcellular compartmentalization of two calcium binding proteins, calretinin and calbindin- $28 \mathrm{kDa}$, in ganglion and amacrine cells of the rat retina. Mol Vis 14:1600-1613.

Molnar E, Pickard L, Duckworth JK 2002. Developmental changes in ionotropic glutamate receptors: lessons from hippocampal synapses. Neuroscientist 8:143-153.

Morris BJ 1997. Stabilization of dendritic mRNAs by nitric oxide allows localized, activitydependent enhancement of hippocampal protein synthesis. Eur J Neurosci 9:2334-2339.

Mulholland PJ, Chandler LJ 2007. The thorny side of addiction: adaptive plasticity and dendritic spines. ScientificWorldJournal 7:9-21.

Munton RP, Tweedie-Cullen R, Livingstone-Zatchej M, Weinandy F, Waidelich M, Longo D, Gehrig P, Potthast F, Rutishauser D, Gerrits B, Panse C, Schlapbach R, Mansuy IM 2007. Qualitative and quantitative analyses of protein phosphorylation in naive and stimulated mouse synaptosomal preparations. Mol Cell Proteomics 6:283-293.

Neuman-Silberberg FS, Schupbach T 1993. The Drosophila dorsoventral patterning gene gurken produces a dorsally localized RNA and encodes a TGF alpha-like protein. Cell 75:165-174.

Nicoll RA, Schmitz D 2005. Synaptic plasticity at hippocampal mossy fibre synapses. Nat Rev Neurosci 6:863-876.

Oertner TG, Matus A 2005. Calcium regulation of actin dynamics in dendritic spines. Cell Calcium 37:477-482. 
Oh MC, Derkach VA, Guire ES, Soderling TR 2006. Extrasynaptic membrane trafficking regulated by GluR1 serine 845 phosphorylation primes AMPA receptors for long-term potentiation. J Biol Chem 281:752-758.

Okabe S, Shiomura Y, Hirokawa N 1989. Immunocytochemical localization of microtubule-associated proteins $1 \mathrm{~A}$ and 2 in the rat retina. Brain Res 483:335-346.

Okada T, Schultz K, Geurtz W, Hatt H, Weiler R 1999. AMPA-preferring receptors with high $\mathrm{Ca} 2+$ permeability mediate dendritic plasticity of retinal horizontal cells. Eur $\mathrm{J}$ Neurosci 11:1085-1095.

Oliveira CS, Rigon AP, Leal RB, Rossi FM 2008. The activation of ERK1/2 and p38 mitogen-activated protein kinases is dynamically regulated in the developing rat visual system. Int J Dev Neurosci 26:355-362.

Olney JW 1968. An electron microscopic study of synapse formation, receptor outer segment development, and other aspects of developing mouse retina. Invest Ophthalmol 7:250-268.

Olson WP 1998. Prions: a review, theories, and proposals. PDA J Pharm Sci Technol 52:134-146.

Orban PC, Chapman PF, Brambilla R 1999. Is the Ras-MAPK signalling pathway necessary for long-term memory formation? Trends Neurosci 22:38-44.

Paris J, Swenson K, Piwnica-Worms H, Richter JD 1991. Maturation-specific polyadenylation: in vitro activation by $\mathrm{p} 34 \mathrm{cdc} 2$ and phosphorylation of a 58-kD CPEbinding protein. Genes Dev 5:1697-1708.

Peng YW, Hao Y, Petters RM, Wong F 2000. Ectopic synaptogenesis in the mammalian retina caused by rod photoreceptor-specific mutations. Nat Neurosci 3:1121-1127.

Peng YW, Senda T, Hao Y, Matsuno K, Wong F 2003. Ectopic synaptogenesis during retinal degeneration in the royal college of surgeons rat. Neuroscience 119:813-820.

Pollock GS, Vernon E, Forbes ME, Yan Q, Ma YT, Hsieh T, Robichon R, Frost DO, Johnson JE 2001. Effects of early visual experience and diurnal rhythms on BDNF mRNA and protein levels in the visual system, hippocampus, and cerebellum. J Neurosci 21:3923-3931.

Poncer JC, Esteban JA, Malinow R 2002. Multiple mechanisms for the potentiation of AMPA receptor-mediated transmission by alpha-Ca2+/calmodulin-dependent protein kinase II. J Neurosci 22:4406-4411.

Pokrywka NJ, Stephenson EC 1991. Microtubules mediate the localization of bicoid RNA during Drosophila oogenesis. Development 113:55-66.

Pourcho RG, Osman K 1986. Cytochemical identification of cholinergic amacrine cells in cat retina. J Comp Neurol 247:497-504. 
Rich KA, Zhan Y, Blanks JC 1997. Migration and synaptogenesis of cone photoreceptors in the developing mouse retina. J Comp Neurol 388:47-63.

Richter JD 1991. Translational control during early development. Bioessays. 13(4):179183.

Richter JD 2007. CPEB: a life in translation. Trends Biochem Sci 32:279-285.

Roberts LA, Large CH, Higgins MJ, Stone TW, O'Shaughnessy CT, Morris BJ 1998. Increased expression of dendritic mRNA following the induction of long-term potentiation. Brain Res Mol Brain Res 56:38-44.

Rockhill RL, Daly FJ, MacNeil MA, Brown SP, Masland RH 2002. The diversity of ganglion cells in a mammalian retina. $\mathrm{J}$ Neurosci 22:3831-3843.

Rodieck RW (1998) The first steps in seeing. Sunderland, MA; Sinauer Associs, Inc.

Ronesi JA, Huber KM 2008. Metabotropic glutamate receptors and fragile $x$ mental retardation protein: partners in translational regulation at the synapse. Sci Signal 1(5):14.

Rosen LB, Ginty DD, Weber MJ, Greenberg ME 1994. Membrane depolarization and calcium influx stimulate MEK and MAP kinase via activation of Ras. Neuron 12:12071221.

Rosenmund C, Clements JD, Westbrook GL 1993. Nonuniform probability of glutamate release at a hippocampal synapse. Science 262(5134):754-757.

Rozov A, Zilberter Y, Wollmuth LP, Burnashev N 1998. Facilitation of currents through rat $\mathrm{Ca} 2+-$ permeable AMPA receptor channels by activity-dependent relief from polyamine block. J Physiol 511 (2):361-377.

Sala R, Viegi A, Rossi FM, Pizzorusso T, Bonanno G, Raiteri M, Maffei L 1998. Nerve growth factor and brain-derived neurotrophic factor increase neurotransmitter release in the rat visual cortex. Eur J Neurosci 10(6):2185-2191.

Sallés FJ, Richards WG, Strickland S 1999. Assaying the polyadenylation state of mRNAs. Methods 17:38-45.

Sassoe-Pognetto $M$, Wassle $H$ 1997. Synaptogenesis in the rat retina: subcellular localization of glycine receptors, GABA(A) receptors, and the anchoring protein gephyrin. J Comp Neurol 381:158-174.

Schmidt M, Wassle H, Humphrey M 1985. Number and distribution of putative cholinergic neurons in the cat retina. Neurosci Lett 59:235-240.

Schmitz F, Holbach M, Drenckhahn D 1993. Colocalization of retinal dystrophin and actin in postsynaptic dendrites of rod and cone photoreceptor synapses. Histochemistry 100:473-479. 
Schnapp BJ, Arn EA, Deshler JO, Highett MI 1997. RNA localization in Xenopus oocytes. Semin Cell Dev Biol 8:529-540.

Schubert V, Dotti CG 2007. Transmitting on actin: synaptic control of dendritic architecture. J Cell Sci 120:205-212.

Sekino Y, Kojima N, Shirao T 2007. Role of actin cytoskeleton in dendritic spine morphogenesis. Neurochem Int 51:92-104.

Selcher JC, Atkins CM, Trzaskos JM, Paylor R, Sweatt JD 1999. A necessity for MAP kinase activation in mammalian spatial learning. Learn Mem 6:478-490.

Shepherd JD, Huganir RL 2007. The cell biology of synaptic plasticity: AMPA receptor trafficking. Annu Rev Cell Dev Biol 23:613-643.

Sherry DM, Wang MM, Bates J, Frishman LJ 2003. Expression of vesicular glutamate transporter 1 in the mouse retina reveals temporal ordering in development of rod vs. cone and ON vs. OFF circuits. J Comp Neurol 465:480-498.

Shigemoto R, Kinoshita A, Wada E, Nomura S, Ohishi H, Takada M, Flor PJ, Neki A, Abe T, Nakanishi S, Mizuno N 1997. Differential presynaptic localization of metabotropic glutamate receptor subtypes in the rat hippocampus. J Neurosci 17:7503-7522.

Shin CY, Kundel M, Wells DG 2004. Rapid, activity-induced increase in tissue plasminogen activator is mediated by metabotropic glutamate receptor-dependent mRNA translation. J Neurosci 24(42):9425-9433.

Si K, Giustetto M, Etkin A, Hsu R, Janisiewicz AM, Miniaci MC, Kim JH, Zhu H, Kandel ER 2003a. A neuronal isoform of CPEB regulates local protein synthesis and stabilizes synapse-specific long-term facilitation in Aplysia. Cell 115:893-904.

Si K, Lindquist S, Kandel ER 2003b. A neuronal isoform of the Aplysia CPEB has prionlike properties. Cell 115:879-891.

Silva AJ 2003. Molecular and cellular cognitive studies of the role of synaptic plasticity in memory. J Neurobiol 54:224-237.

Silva AJ, Paylor R, Wehner JM, Tonegawa S 1992a. Impaired spatial learning in alphacalcium-calmodulin kinase II mutant mice. Science 257:206-211.

Silva AJ, Stevens CF, Tonegawa S, Wang Y 1992b. Deficient hippocampal long-term potentiation in alpha-calcium-calmodulin kinase II mutant mice. Science 257:201-206.

Simon P, Schott K, Williams RW, Schaeffel F 2004. Posttranscriptional regulation of the immediate-early gene EGR1 by light in the mouse retina. Eur J Neurosci 20:3371-3377.

Simon R, Tassan JP, Richter JD 1992. Translational control by poly(A) elongation during Xenopus development: differential repression and enhancement by a novel cytoplasmic polyadenylation element. Genes Dev 6:2580-2591. 
Skeberdis VA, Lan J, Opitz T, Zheng X, Bennett MV, Zukin RS 2001. mGluR1-mediated potentiation of NMDA receptors involves a rise in intracellular calcium and activation of protein kinase C. Neuropharmacology 40:856-865.

Soderling TR 2000. CaM-kinases: modulators of synaptic plasticity. Curr Opin Neurobiol 10:375-380.

Soderling TR, Chang B, Brickey D 2001. Cellular signaling through multifunctional Ca2+/calmodulin-dependent protein kinase II. J Biol Chem 276:3719-3722.

Soule J, Messaoudi E, Bramham CR 2006. Brain-derived neurotrophic factor and control of synaptic consolidation in the adult brain. Biochem Soc Trans 34:600-604.

Specht D, Tom DS, Ammermuller J, Regus-Leidig H, Gundelfinger ED, Brandstatter JH 2007. Structural and functional remodeling in the retina of a mouse with a photoreceptor synaptopathy: plasticity in the rod and degeneration in the cone system. Eur J Neurosci 26:2506-2515.

St JD, Nusslein-Volhard C 1992. The origin of pattern and polarity in the Drosophila embryo. Cell 68:201-219.

Stebbins-Boaz B, Cao Q, de Moor CH, Mendez R, Richter JD 1999. Maskin is a CPEBassociated factor that transiently interacts with elF-4E. Mol Cell 4:1017-1027.

Steve Olson 2004. Prions on my mind - in "the synapse revealed". HHMI bulletin, Fall 2004: 14-23.

Steward O, Wallace CS, Lyford GL, Worley PF 1998. Synaptic activation causes the mRNA for the IEG Arc to localize selectively near activated postsynaptic sites on dendrites. Neuron 21:741-751.

Steward O, Schuman EM 2001. Protein synthesis at synaptic sites on dendrites. Annu Rev Neurosci 24:299-325.

Strettoi E, Mears AJ, Swaroop A 2004. Recruitment of the rod pathway by cones in the absence of rods. J Neurosci 24:7576-7582.

Strettoi E, Pignatelli V 2000. Modifications of retinal neurons in a mouse model of retinitis pigmentosa. Proc Natl Acad Sci USA 97:11020-11025.

Strettoi E, Raviola E, Dacheux RF 1992. Synaptic connections of the narrow-field, bistratified rod amacrine cell (All) in the rabbit retina. J Comp Neurol 325:152-168.

Sullivan RK, Woldemussie E, Pow DV 2007. Dendritic and synaptic plasticity of neurons in the human age-related macular degeneration retina. Invest Ophthalmol Vis Sci 48:2782-2791.

Swope SL, Moss SJ, Raymond LA, Huganir RL 1999. Regulation of ligand-gated ion channels by protein phosphorylation. Adv Second Messenger Phosphoprotein Res 33:49-78. 
Taha S, Hanover JL, Silva AJ, Stryker MP 2002. Autophosphorylation of alphaCaMKII is required for ocular dominance plasticity. Neuron 36:483-491.

Takahashi H, Sekino Y, Tanaka S, Mizui T, Kishi S, Shirao T 2003. Drebrin-dependent actin clustering in dendritic filopodia governs synaptic targeting of postsynaptic density95 and dendritic spine morphogenesis. J Neurosci 23:6586-6595.

Tanaka H, Grooms SY, Bennett MV, Zukin RS 2000. The AMPAR subunit GluR2: still front and center-stage. Brain Res 886:190-207.

Terashima T, Ochiishi T, Yamauchi T 1994. Immunocytochemical localization of calcium/calmodulin-dependent protein kinase II isoforms in the ganglion cells of the rat retina: immunofluorescence histochemistry combined with a fluorescent retrograde tracer. Brain Res 650:133-139.

Theis M, Si K, Kandel ER 2003. Two previously undescribed members of the mouse CPEB family of genes and their inducible expression in the principal cell layers of the hippocampus. Proc Natl Acad Sci USA 100:9602-9607.

Theurkauf WE, Smiley S, Wong ML, Alberts BM 1992. Reorganization of the cytoskeleton during Drosophila oogenesis: implications for axis specification and intercellular transport. Development 115:923-936.

Tian N, Copenhagen DR 2001. Visual deprivation alters development of synaptic function in inner retina after eye opening. Neuron 32:439-449.

Tian N, Copenhagen DR 2003. Visual stimulation is required for refinement of ON and OFF pathways in postnatal retina. Neuron 39:85-96.

Tominaga-Yoshino K, Urakubo T, Okada M, Matsuda H, Ogura A 2008. - Repetitive induction of late-phase LTP produces long-lasting synaptic enhancement accompanied by synaptogenesis in cultured hippocampal slices. Hippocampus 18(3):281-293.

Tong G, Takahashi H, Tu S, Shin Y, Talantova M, Zago W, Xia P, Nie Z, Goetz T, Zhang D, Lipton SA, Nakanishi N 2008. Modulation of NMDA receptor properties and synaptic transmission by the NR3A subunit in mouse hippocampal and cerebrocortical neurons. $J$ Neurophysiol 99:122-132.

Tongiorgi E, Righi M, Cattaneo A 1996. Subcellular localisation of neurotrophins and neurotrophin receptors: implications for synaptic plasticity. Rev Bras Biol 56 Su $1 \mathrm{Pt}$ 1:175-182.

Tongiorgi E, Domenici L, Simonato M 2006. What is the biological significance of BDNF mRNA targeting in the dendrites? Clues from epilepsy and cortical development. Mol Neurobiol 33:17-32.

Tucker RP, Matus Al 1988. Microtubule-associated proteins characteristic of embryonic brain are found in the adult mammalian retina. Dev Biol 130:423-434.

Tumosa N, Eckenstein F, Stell WK 1984. Immunocytochemical localization of putative cholinergic neurons in the goldfish retina. Neurosci Lett 48:255-259. 
Tumosa N, Stell WK 1986. Choline acetyltransferase immunoreactivity suggests that ganglion cells in the goldfish retina are not cholinergic. J Comp Neurol 244:267-275.

Turner DL, Cepko CL 1987. A common progenitor for neurons and glia persists in rat retina late in development. Nature 328:131-136.

Vassalli JD, Huarte J, Belin D, Gubler P, Vassalli A, O'Connell ML, Parton LA, Rickles RJ, Strickland S 1989. Regulated polyadenylation controls mRNA translation during meiotic maturation of mouse oocytes. Genes Dev 3:2163-2171.

Vecino E, Hernandez M, Garcia M 2004. Cell death in the developing vertebrate retina. Int J Dev Biol 48:965-974.

Vistamehr S, Tian N 2004. Light deprivation suppresses the light response of inner retina in both young and adult mouse. Vis Neurosci 21:23-37.

Voigt T 1986. Cholinergic amacrine cells in the rat retina. J Comp Neurol 248:19-35.

Wada A, Takahashi H, Lipton SA, Chen HS 2006. NR3A modulates the outer vestibule of the "NMDA" receptor channel. J Neurosci 26:13156-13166.

Walker J, Minshall N, Hake L, Richter J, Standart N 1999. The clam 3' UTR masking element-binding protein p82 is a member of the CPEB family. RNA 5:14-26.

Waltereit R, Weller M 2003. Signaling from CAMP/PKA to MAPK and synaptic plasticity. Mol Neurobiol 27:99-106.

Wang JQ, Arora A, Yang L, Parelkar NK, Zhang G, Liu X, Choe ES, Mao L 2005. Phosphorylation of AMPA receptors: mechanisms and synaptic plasticity. Mol Neurobiol 32:237-249.

Wang JQ, Liu X, Zhang G, Parelkar NK, Arora A, Haines M, Fibuch EE, Mao L 2006. Phosphorylation of glutamate receptors: a potential mechanism for the regulation of receptor function and psychostimulant action. J Neurosci Res 84:1621-1629.

Wang QP, Jammoul F, Duboc A, Gong J, Simonutti M, Dubus E, Craft CM, Ye W, Sahel JA, Picaud S 2008. Treatment of epilepsy: the GABA-transaminase inhibitor, vigabatrin, induces neuronal plasticity in the mouse retina. Eur J Neurosci 27:2177-2187.

Weiler R, Schultz K, Janssen-Bienhold U 1995. Retraction of spinule-type neurites from carp retinal horizontal cell dendrites during dark adaptation involves the activation of Ca2+/calmodulin-dependent protein kinase II. Eur J Neurosci 7:1914-1919.

Weiler R, Schultz K, Janssen-Bienhold U 1996. $\mathrm{Ca}(2+)$-dependency of spinule plasticity at dendrites of retinal horizontal cells and its possible implication for the functional role of spinules. Vision Res 36:3891-3900.

Wells DG, Richter JD, Fallon JR 2000. Molecular mechanisms for activity-regulated protein synthesis in the synapto-dendritic compartment. Curr Opin Neurobiol 10:132-137. 
Westbrook GL, Mayer ML 1987. Micromolar concentrations of Zn2+ antagonize NMDA and GABA responses of hippocampal neurons. Nature 328:640-643.

Wilt FH 1973. Polyadenylation of maternal RNA of sea urchin eggs after fertilization. Proc Natl Acad Sci USA 70(8):2345-2349.

Wong RO, Ghosh A 2002. Activity-dependent regulation of dendritic growth and patterning. Nat Rev Neurosci 3:803-812.

Wong WT, Faulkner-Jones BE, Sanes JR, Wong RO 2000. Rapid dendritic remodeling in the developing retina: dependence on neurotransmission and reciprocal regulation by Rac and Rho. J Neurosci 20:5024-5036.

Wu L, Wells D, Tay J, Mendis D, Abbott MA, Barnitt A, Quinlan E, Heynen A, Fallon JR, Richter JD 1998. CPEB-mediated cytoplasmic polyadenylation and the regulation of experience-dependent translation of alpha-CaMKII mRNA at synapses. Neuron 21:11291139.

Xia Y, Carroll RC, Nawy S 2006. State-dependent AMPA receptor trafficking in the mammalian retina. J Neurosci 26:5028-5036.

Xia Y, Nawy S, Carroll RC 2007. Activity-dependent synaptic plasticity in retinal ganglion cells. J Neurosci 27:12221-12229.

Xue J, Cooper NG 2001. The modification of NMDA receptors by visual experience in the rat retina is age dependent. Brain Res Mol Brain Res 91:196-203.

Xue J, Li G, Laabich A, Cooper NG 2001. Visual-mediated regulation of retinal CaMKII and its GluR1 substrate is age-dependent. Brain Res Mol Brain Res 93:95-104.

Yamagata M, Sanes JR 1995a. Lamina-specific cues guide outgrowth and arborization of retinal axons in the optic tectum. Development 121:189-200.

Yamagata M, Sanes JR 1995b. Target-independent diversification and target-specific projection of chemically defined retinal ganglion cell subsets. Development 121:37633776.

Yashiro K, Corlew R, Philpot BD 2005. Visual deprivation modifies both presynaptic glutamate release and the composition of perisynaptic/extrasynaptic NMDA receptors in adult visual cortex. J Neurosci 14;25(50):11684-11692.

Yin Y, Edelman GM, Vanderklish PW 2002. The brain-derived neurotrophic factor enhances synthesis of Arc in synaptoneurosomes. Proc Natl Acad Sci U S A 99:23682373.

Young RW 1985a. Cell differentiation in the retina of the mouse. Anat Rec 212:199-205.

Young RW 1984. Cell death during differentiation of the retina in the mouse. J Comp Neurol 229:362-373. 
Young RW 1985b. Cell proliferation during postnatal development of the retina in the mouse. Brain Res 353:229-239.

Zhang J, Yang Z, Wu SM 2005. Development of cholinergic amacrine cells is visual activity-dependent in the postnatal mouse retina. J Comp Neurol 484:331-343.

Zhou M, Baudry M 2006. Developmental changes in NMDA neurotoxicity reflect developmental changes in subunit composition of NMDA receptors. J Neurosci 26:29562963.

Zucker CL, Ehinger B 1998. Gamma-aminobutyric acidA receptors on a bistratified amacrine cell type in the rabbit retina. J Comp Neurol 393:309-319. 


\section{APPENDIX: LIST OF ABBREVIATIONS}

ABP

APB

ApCPEB

AMD

AMPA

AMPAR

AP

ARVO

B-PER

CA1

CA3

ChAT

BDNF

CaMK

CaMKII

CaMKIla

CaMKIlaB

CPE

CPEB

CNS
AMPA receptor binding protein

2-amino-4-phosphonobutyrate

Aplysia CPEB

age-related macular degeneration

a-amino-3-hydroxy-5-methyl-4-isoxazole propionate

$\alpha$-amino-3-hydroxy-5-methyl-4-isoxazole propionate receptor

anterior-posterior

Bacterial Protein Extraction Buffer

Cornu Ammonis area 1

Cornu Ammonis area 3

choline acetyl transferase

Brain-derived neurotrophic factor

$\mathrm{Ca}^{2+} /$ calmodulin-dependent protein kinases

multifunctional $\mathrm{Ca}^{2+} /$ calmodulin-dependent protein kinases

multifunctional $\mathrm{Ca}^{2+} /$ calmodulin-dependent protein kinases, a chain

multifunctional $\mathrm{Ca}^{2+} /$ calmodulin-dependent protein kinases, $\alpha$ chain isoform $B$

cytoplasmic polyadenylation element

cytoplasmic polyadenylation element binding protein

central nervous system 
CPSF Polyadenylation Specific Factor

DV dorsal-ventral

ERG electroretinogram

elF4E eukaryotic translational initiation factor $4 \mathrm{E}$

elF4G eukaryotic translational initiation factor $4 G$

EGR1 early growth response factor-1

ELK2 a potassium channel

ERK extracellular signal-regulated kinase

EPSP excitatory post synaptic potential

FAM 6-carboxyfluorescein

FMRP Fragile-X Mental Retardation Proteins

GABA gamma-aminobutyric acid

GEF guanine exchange factor

GluR1-4 AMPA medated glutamate receptor subunit 1-4

GluR5-7 kainate receptor subunit 1-3

GRIA1-4 Glutamate receptor, ionotropic, AMPA mediated, subunit 1-4

GRIN1-3 Glutamate receptor, ionotropic, NMDA mediated, subunit 1-3

GRIK1-5 Glutamate receptor, ionotropic, kainate mediated, subunit 1-5

GST glutathionine S-transferase

IACUC Institutional Animal Care and Use Committee

iGluR ionotropic glutamate receptors

INL inner nuclear layer

IPL inner plexiform layer

IPTG $\quad$ isopropyl $\beta-D-1$-thiogalactopyranoside

IRBP interstitial retinol binding protein

KA1-2 kainate receptor subunit 4-5 


\begin{tabular}{|c|c|}
\hline KAR & kainate receptor \\
\hline LM-PAT & ligation-mediated polyadenylation test \\
\hline LTD & long-term depression \\
\hline LTP & long-term potentiation \\
\hline MAP1a & microtubule-associated protein $1 \mathrm{a}$ \\
\hline MAP2 & microtubule-associated protein 2 \\
\hline MAPK & mitogen-activated protein kinase \\
\hline MAPKK & MAP kinase kinase \\
\hline MAPKKK & MAP kinase kinase kinase \\
\hline MEK & MAP/ERK kinase \\
\hline MF & mossy fiber (of hippocampus) \\
\hline mGluR & metabotropic glutamate receptor \\
\hline mGluR1-5 & metabotropic glutamate receptor subunit $1-5$ \\
\hline NMDA & $\mathrm{N}$-methyl-D-aspartate \\
\hline NMDAR & $\mathrm{N}$-methyl-D-aspartate receptor \\
\hline NR1-3 & NMDA receptor subunit 1-3 \\
\hline $\mathrm{NR} 2 \mathrm{~A}, 2 \mathrm{~B}$ & NMDA receptor subunit 2, isoformA, B \\
\hline ONL & outer nuclear layer \\
\hline OP & oscillatory potentials \\
\hline OPL & outer plexiform layer \\
\hline PABP & poly $(A)$ binding protein \\
\hline PAGE & polyacrylamide gel electrophoresis \\
\hline PAP & $\operatorname{poly}(A)$ polymerase \\
\hline PAS & polyadenylation signal \\
\hline PAT & polyadenylation test \\
\hline PCR & polymerase chain reaction \\
\hline
\end{tabular}




\begin{tabular}{ll} 
PICK1 & Protein Interacting with C-Kinase 1 \\
PKA & protein kinase A \\
PKC & protein kinase C \\
Ppp2r1a & protein phosphatase 2, regulatory domain 1a \\
Ppp2r2b & protein phosphatase 2, regulatory domain 2b \\
proBDNF & the unprocessed pro-form of BDNF \\
PSD & post synaptic density \\
PVDF & Polyvinylidene Fluoride \\
RACE & rapid amplification of cDNA ends \\
RBD & RNA binding domain \\
RCM3 & rat calmodulin \\
rdta & rod-ablated model \\
RGC & retinal ganglion cell layer \\
RACE & rapid amplification of cDNA ends \\
RPE & retinal pigment epithelium \\
RT & reverse transcription \\
RRF & RNA recognition motif \\
SDS & sodium dodecyl sulfate \\
SELEX & systermatic evolution of ligands by exponential enrichment \\
siRNA & small interference RNA \\
synGAP & synaptic Ras-GTPase activating protein \\
t-PA & tissue-type plasminogen activator \\
TTR & transthyretin \\
UTR & untranslated region \\
\hline
\end{tabular}




\section{CURRICULUM VITAE}

NAME: $\quad$ Xiangping Wang

ADDRESS: Department of Anatomical Sciences and Neurobiology

School of Medicine

500 S Preston Street

University of Louisville

Louisville, KY 40292

TRAINING:

1993-1997 B.S. Biochemistry and Molecular Biology, Peking University, Beijing, China

2001-2004 M.S. Anatomical Sciences and Neurobiology, University of Louisville, KY

2004-2008 Ph.D. Anatomical Sciences and Neurobiology, University of Louisville, KY

POSITIONS:

2003 Teaching Assistant/Graduate student, Medical Neuroscience

2004-2008 Research Assistant/Graduate student, Department of Anatomical

Sciences and Neurobiology, University of Louisville, Louisville, KY

\section{PROFESSIONAL SOCIETY MEMBERSHIPS:}

2001- Society for Neuroscience

2004- The Association for Research in Vision and Ophthalmology (ARVO)

HONORS AND AWARDS:

2006 Travel Awards, the first annual IDeA meeting, Washington D.C. Abstract "3' UTR and CPEB-Mediated Translational Control during Development of the Neural Retina"

2006 Travel Awards, Southeast Nerve Net (SENN) conference, Atlanta, GA. Oral presentation: "The Rhythm of BDNF expression during diurnal cycles in mouse retina" 


\section{FULL LENGTH PAPERS}

Baldauf ZB, Wang XP, Wang S, Bickford ME 2003. Pretectotectal pathway: an ultrastructural quantitative analysis in cats. J Comp Neurol 464(2):141-158.

Cooper NG, Laabich A, Fan W, Wang X 2008. The relationship between neurotrophic factors and CaMKII in the death and survival of retinal ganglion cells. Prog Brain Res 173:521-540.

Rouchka EC, Wang XP, Graham JH, Cooper NG 2009. Computational prediction of genes translationally regulated by cytoplasmic polyadenylation elements.

$1^{\text {st }}$ international Conference on Bioinformatics and Computational Biology (BICoB), New Orleans, USA.

\section{ABSTRACTS}

2006 The first annual Institutional Development Award (IdeA) conference, Washington D.C. Abstract: 3' UTR and CPEB-Mediated Translational Control during Development of the Neural Retina

2006 UT-ORNL-KBRIN Bioinformatics Summit, Cadiz, KY. Abstract: Computational Detection of CPE Motifs in 3' UTR Sequences

2006 Society for Neuroscience Conference, Atlanta, GA. Abstract: Computatinal Screening of CPE motifs in $3^{\prime}$ UTR and CPEB-Mediated Translational Control during Development of the Neural Retina

2007 Society for Neuroscience Conference, San Diego, CA. Abstract: CPEB3 mediated translational control in the mouse retina

2008

The second annual Institutional Development Award (IdeA) conference, Washington D.C. Abstract: CPEB3: A Translational Regulation Molecule in the Adult Retina

2008

Society for Neuroscience Conference, Washington D.C. Abstract: The Implications for CPEB3 in the Developing Retina

ORAL PRESENTATION 